\title{
Development of Improved Acellular Pertussis Vaccines Through Inclusion of the RTX Antigen or Induction of Mucosal Immunity
}

\author{
Dylan Tyler Boehm \\ West Virginia University, dtboehm@mix.wvu.edu
}

Follow this and additional works at: https://researchrepository.wvu.edu/etd

Part of the Bacterial Infections and Mycoses Commons, Medical Immunology Commons, Medical Microbiology Commons, and the Respiratory Tract Diseases Commons

\section{Recommended Citation}

Boehm, Dylan Tyler, "Development of Improved Acellular Pertussis Vaccines Through Inclusion of the RTX Antigen or Induction of Mucosal Immunity" (2019). Graduate Theses, Dissertations, and Problem Reports. 4049.

https://researchrepository.wvu.edu/etd/4049

This Dissertation is protected by copyright and/or related rights. It has been brought to you by the The Research Repository @ WVU with permission from the rights-holder(s). You are free to use this Dissertation in any way that is permitted by the copyright and related rights legislation that applies to your use. For other uses you must obtain permission from the rights-holder(s) directly, unless additional rights are indicated by a Creative Commons license in the record and/ or on the work itself. This Dissertation has been accepted for inclusion in WVU Graduate Theses, Dissertations, and Problem Reports collection by an authorized administrator of The Research Repository @ WVU.

For more information, please contact researchrepository@mail.wvu.edu. 


\title{
DEVELOPMENT OF IMPROVED ACELLULAR PERTUSSIS VACCINES THROUGH INCLUSION OF THE RTX ANTIGEN OR INDUCTION OF MUCOSAL IMMUNITY
}

\author{
Dylan Tyler Boehm \\ Dissertation submitted to the School of Medicine \\ at West Virginia University \\ in partial fulfillment of the requirements \\ for the degree of \\ Doctor of Philosophy \\ in \\ Immunology and Microbial Pathogenesis
}

F. Heath Damron, Ph.D., Committee Chairperson

Slawomir Lukomski, Ph.D.

Gordon P. Meares, Ph. D.

Rosana Schafer, Ph.D.

Michael D. Schaller, Ph.D.

Department of Microbiology, Immunology, and Cell Biology

Morgantown, West Virginia

2019

Keywords: B. pertussis, adenylate cyclase toxin, acellular vaccine, pertussis, vaccine-induced mucosal immunity, DTaP, innate immunity, pertussis vaccine model

Copyright 2019 Dylan T. Boehm 


\title{
ABSTRACT
}

\section{DEVELOPMENT OF IMPROVED ACELLULAR PERTUSSIS VACCINES THROUGH INCLUSION OF THE RTX ANTIGEN OR INDUCTION OF MUCOSAL IMMUNITY}

\author{
Dylan T. Boehm
}

Pertussis is a human respiratory disease, primarily caused by the Gram-negative pathogen Bordetella pertussis. The infection is most severe and can be life-threatening in young children and infants where it manifests as a series of paroxysmal coughs. The disease is more commonly known as whooping cough, due to the whoop omitted during a massive inspiratory effort to bring air back into the lungs. Pertussis is a toxin-mediated disease that persists due to an early release of toxins that allow that bacteria to evade the cells of the innate immune response. The inhibition of the host response continues as toxin activity disrupts the signaling generating an adaptive immune response. Fortunately, two generations of vaccines have been developed to combat the spread of the highly contagious disease. The whole-cell pertussis (WP) vaccine was implemented in the 1950s and was successful in controlling cases. However, due to adverse side effects associated with the immunization, wP vaccines were replaced with acellular protein (aP) vaccines absorbed to an alum adjuvant. The second generation aP vaccines induced an immune response capable of neutralizing key virulence factors contained in the vaccine, thereby controlling the symptoms of the disease. Unfortunately, there has been a re-emergence of pertussis incidence in countries using the aP vaccines. Included in this resurgence is an increased number of adolescents and adults with unrecognized pertussis, leading to the potential of adult and adolescent reservoirs of the infection. We reasoned that a pertussis vaccine capable of inducing sterilizing immunity, rather than controlling the symptoms, would decrease the spread of pertussis. In this work, we propose two directions towards this goal. The first was through the neutralization of a key virulence factor not included in any current vaccine formulations, adenylate cyclase toxin (ACT). This was accomplished through the optimization of a mouse immunization and challenge model. In initial experiments using a neutrophil reporter mouse, NeCre luc, we visualized the spatiotemporal localization of neutrophils after $B$. pertussis challenge in wP or aP immunized mice with a vaccine dose known to be protective. In these experiments WP immunized mice elicited an exaggerated immune response, that persisted after clearance of the pathogen, raising concerns to the relevance of the high vaccine dose in characterizing the immune response. In subsequent experiments, we addressed these concerns, by titrating the concentration of a Diphtheria-Tetanus-acellular Pertussis (DTaP) subunit vaccine to a dose that did not induce a protective response in a murine model. In defining a suboptimal protective dose for mice, the additive effects of potential antigens could be evaluated, that were previously masked by the higher concentrations of vaccine. This model was confirmed through the addition of an ACT toxoid, RTX. The inclusion of RTX into a DTaP vaccine produced an antibody response that neutralized the toxin in vitro, and upon challenge with $B$. pertussis reduced the bacterial burden compared to mice only receiving the DTaP vaccine. We continued in the direction of promoting a vaccine- 
induced immune response capable of clearing $B$. pertussis from the respiratory tract in future experiments. We hypothesized that early neutralization of the bacterial toxins through the generation of a mucosal immune response in the respiratory tract would increase the clearance of the pathogen. This was tested using a mucosal, intranasally (IN) administered DTaP vaccine. Administration of DTaP through the IN route induced a $B$. pertussis-specific systemic IgG response, and mucosal IgA response, capable of neutralizing the toxins at the site of infection. Furthermore, IN vaccinated mice reduced the bacterial burden to the same levels of intraperitoneal immunized mice. The work completed in this dissertation demonstrates the protective effects of the antigen RTX when incorporated into a current DTaP vaccine. We then altered the route of administration to an intranasal delivered vaccine, demonstrating the effectiveness of inducing a local mucosal response against $B$. pertussis infection. Together these findings establish a foundation for potential vaccine strategies to suppress the toxinmediated dysregulation of the host response and lead to the more efficient clearance of $B$. pertussis from the respiratory tract. 


\section{DEDICATION}

This work is dedicated to my parents, David and Beth Boehm, who together have shaped me into the person I am today. Thank you for caring for me in times of trouble, celebrating with me in times of joy, and for being unwavering examples of how to live life on the days between. 


\section{ACKNOWLEDGMENTS}

First and foremost, I would like to thank my advisor, Dr. F. Heath Damron, for the countless hours he has invested in my progression first as a graduate student and ultimately as a scientist. I will be forever grateful for the effort you put into my success in the many facets of being a scientist. I would also like to thank Dr. Mariette Barbier, for the many hours you have spent designing, re-designing, performing, helping analyze experiments and so much more. Late nights, weekends, together the mentorship of the two of you knows no bounds. The work that we accomplished together was due to the example you have set for myself and members of the lab. Both in your commitment to science and your students, I could not imagine better role models for a career in academia. Thank you.

To the DB Lab team, without each of your instrument roles, the work described in this dissertation would not have happened. You made coming to lab something to look forward to every morning. To Emel, Jesse, Catherine, Ting, Justin, Willy, Allison, Kelly, Jason, Alex, Caleb, Sebastian, Shree, Claire, Katherine, Aaron, Annalisa, Scott, Emily and Eddie, you have been there through thick and thin, thank for making my time so enjoyable. My lunches will never be the same. I wish you all the best on your next adventures.

Next, I would like to thank my dissertation committee. Thank you for your input in shaping the directions of my projects, and your continued advice throughout my dissertation work.

I would also like to thank the members of the Microbiology, Immunology, and Cell Biology department. During my time as a student in the department I have witnessed the department grow in the number of faculty, students, and funded projects. It was being part of that growth that motivated me to continue to become a better researcher.

I would like to acknowledge a few of the directors of the WVU core facilities. Dr. Kathleen Brundage, Dr. Karen Martin, Dr. Amanda Ammer, and Dr. James Coad, thank you for hours you helped design experiments, train me on equipment, fix the equipment I broke, and optimize my experiments.

Lastly, I would like to thank my friends and family. Without your never-ending support and motivation, I would not be here today. 


\section{TABLE OF CONTENTS}

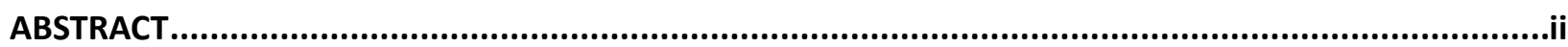

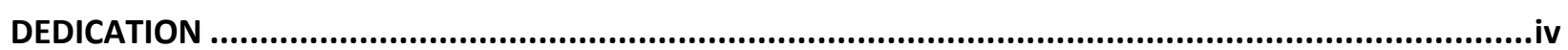

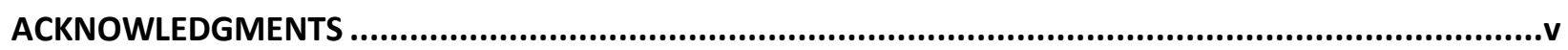

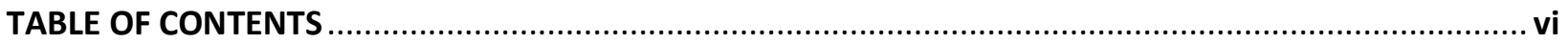

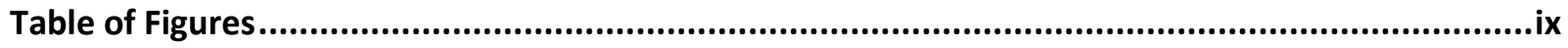

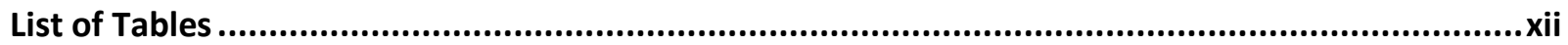

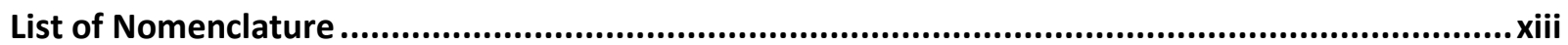

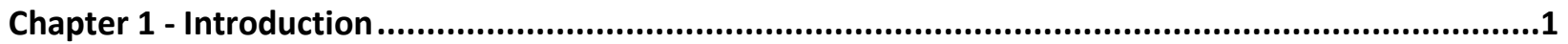

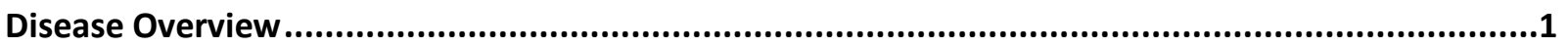

History of B. pertussis vaccination and the increase in B. pertussis incidence ................................2

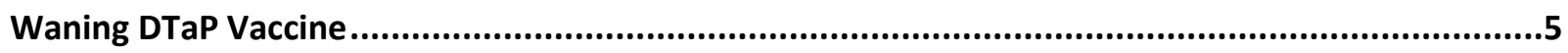

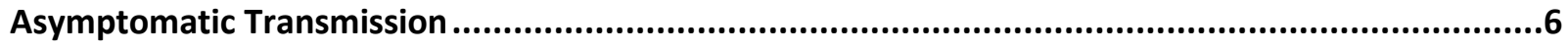

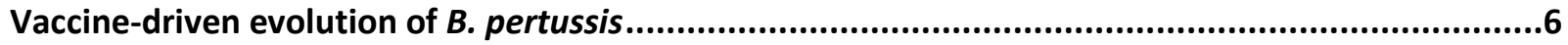

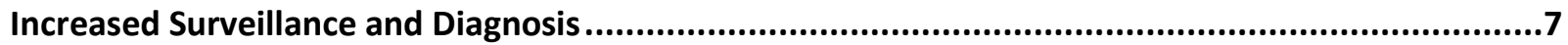

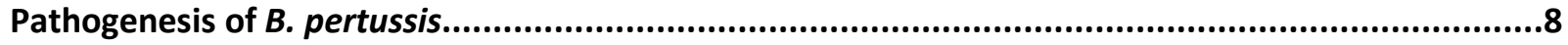

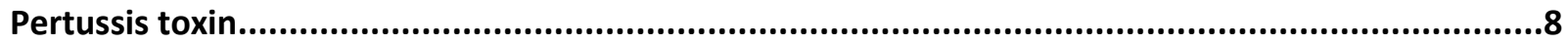

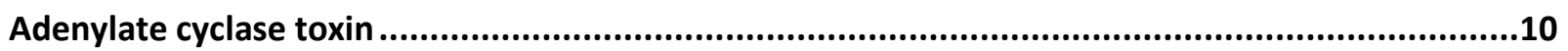

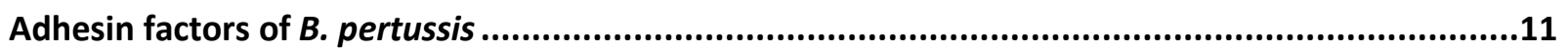

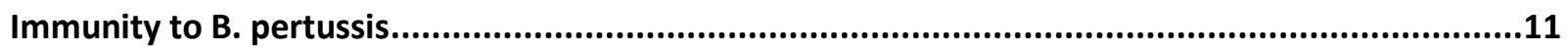

Adaptive immune response

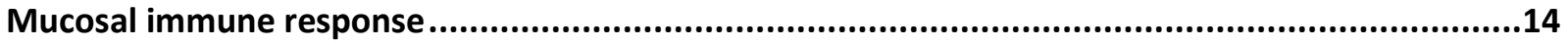

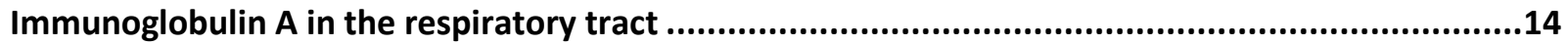

Local cellular mucosal immune response ................................................................................15

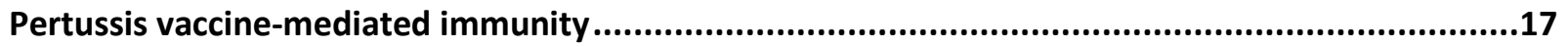

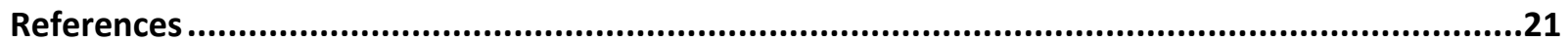

Chapter 2 - Characterizing the innate and adaptive responses of immunized mice to Bordetella pertussis infection using in vivo imaging and transcriptomic analysis .............................................33

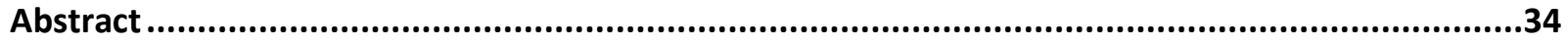

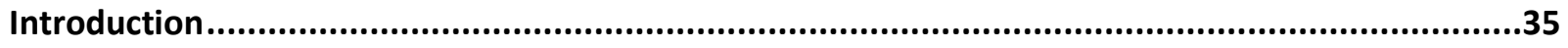

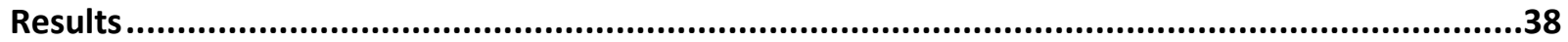




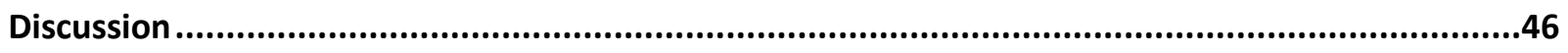

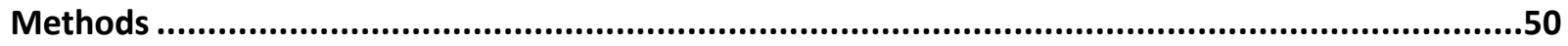

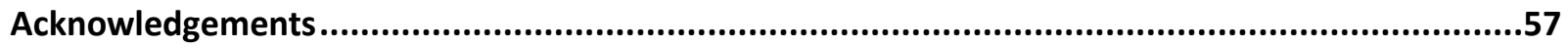

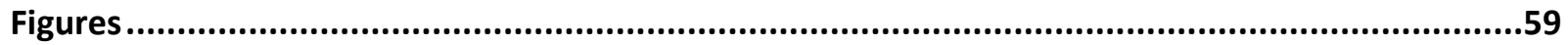

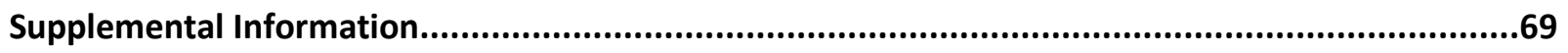

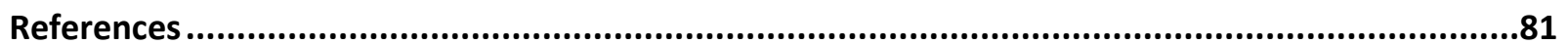

Chapter 3 - Evaluation of adenylate cyclase toxoid antigen in acellular pertussis vaccines using a Bordetella pertussis challenge model in mice................................................................................87

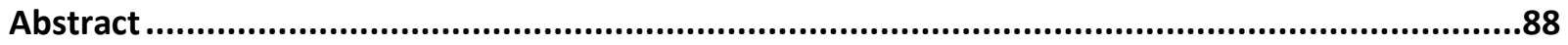

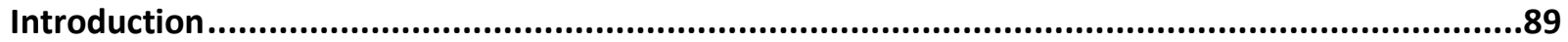

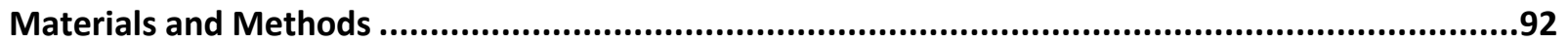

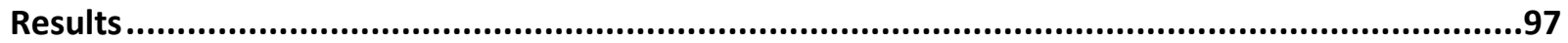

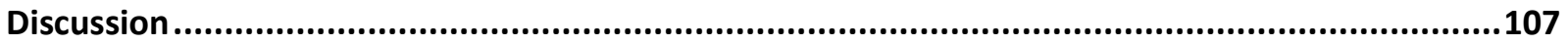

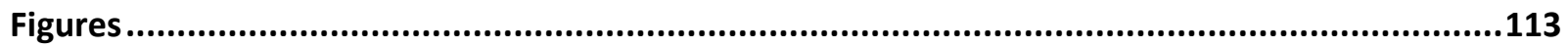

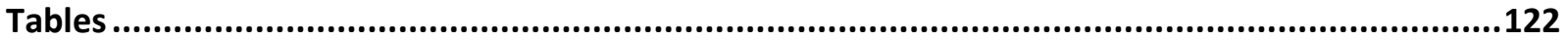

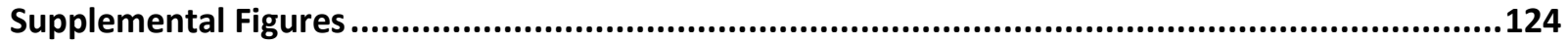

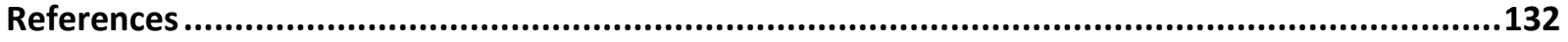

Chapter 4- Intranasal acellular pertussis vaccine provides mucosal immunity and protects mice from

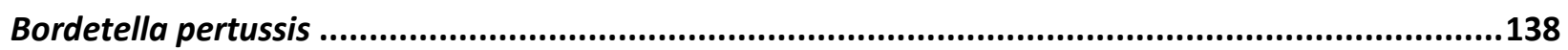

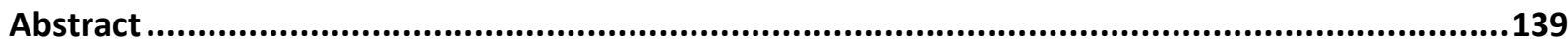

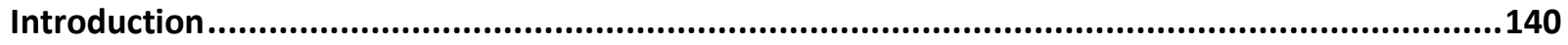

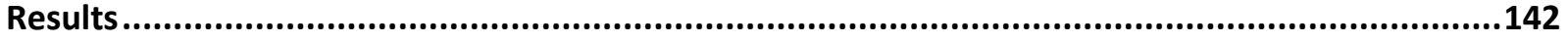

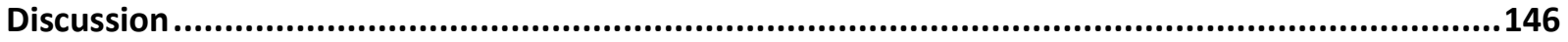

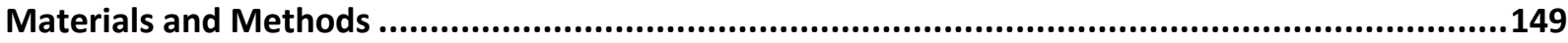

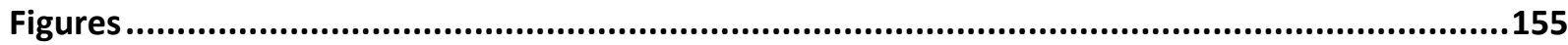

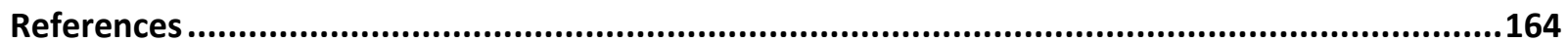

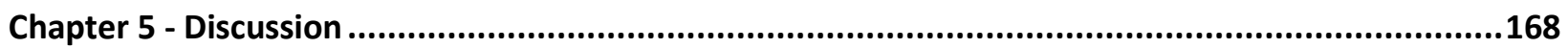

Lessons learned from Ne Cre luc mouse model...................................................................169

Optimization of the mouse model................................................................................................171

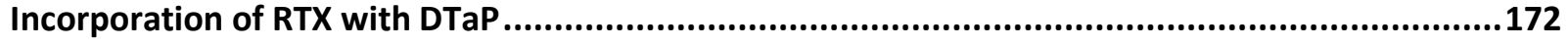

Importance of a Mucosal Response against B. pertussis..........................................................173

Intranasal DTaP induced a protective immune response.........................................................175

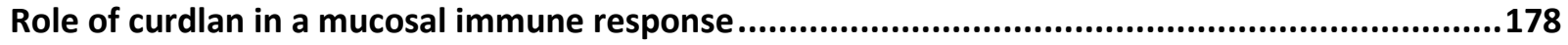

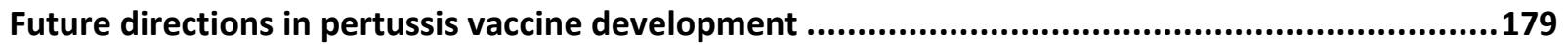


Identification of target vaccine tissue

Importance of nasal cavity specific analysis.

Neutrophils in the nasal cavity, the unsung hero? ..................................................................183

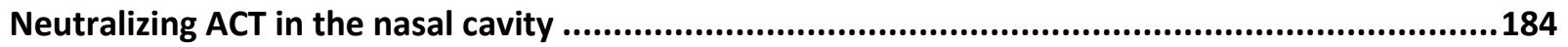

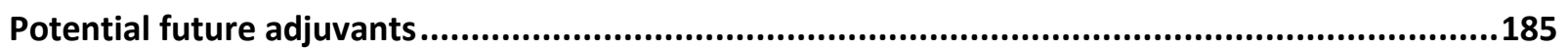

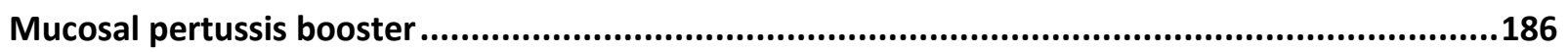

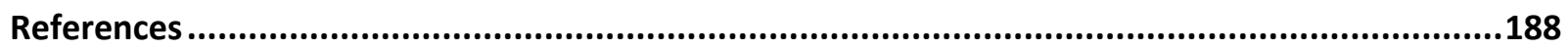




\section{$\underline{\text { Table of Figures }}$}

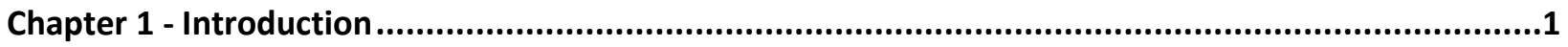

Figure 1: Incidence of clinical B. pertussis cases in the United States from 1922-2017. Data reported from

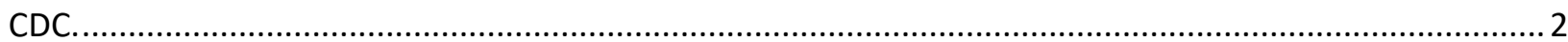

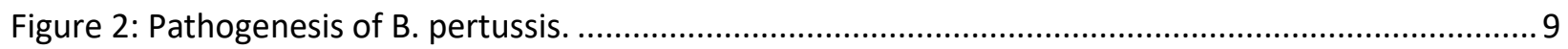

Chapter 2 - Characterizing the innate and adaptive responses of immunized mice to Bordetella pertussis infection using in vivo imaging and transcriptomic analysis..........................................33

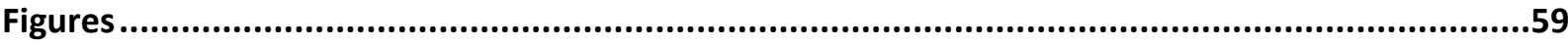

Figure 1: Experimental workflow for vaccination, B. pertussis challenge, sample acquisition, and analysis

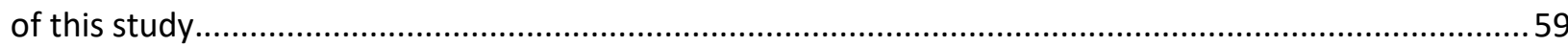

Figure 2: Kaplan- Meier survival curves of NECre luc mice according to immunization group.................60

Figure 3: Bacterial burden in respiratory tissue and serological responses to $B$. pertussis challenge of

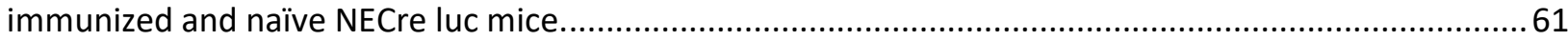

Figure 4: IVIS imaging and flow cytometric analysis of naïve and immunized NECre luc mice post challenge with $B$. pertussis.

Figure 5: Analysis of cytokine profiles from lungs of naïve and immunized NECre luc mice post challenge

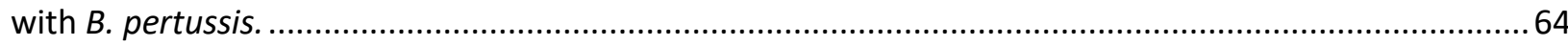

Figure 6: Lung transcriptome profile of vaccinated and challenged NECre luc mice..............................65

Figure 7: Enrichment analysis and relative fold-changes of genes upregulated following $B$. pertussis challenge.

Fig. 8: Differentiated genes associated with neutrophil recruitment at early and late time-points

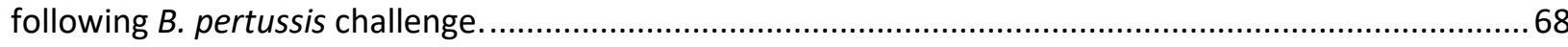

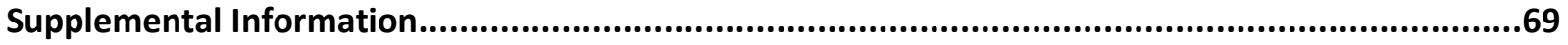

Supplementary Fig. 1: IVIS imaging of naïve and immunized NECre luc mice post challenge with $B p$ as image was acquired from Xenogen IVIS Lumina II.

Supplementary Fig. 2: Representative images and gating strategy for neutrophils from lung and nasal lavage tissues.

Supplementary Fig. 3: Analysis of Treg and Th2 associated cytokines from lungs of naïve and immunized NECre luc mice post challenge with $B$. pertussis

Supplemental Fig. 4: Volcano plot analysis of differentiated genes at early and late time-points of immunized or naïve mice following challenge.

Supplementary Fig. 5: Gene expression profiles of T helper cell immune responses at early time point. 79 Supplementary Fig. 6: Repertoire of B cell clones identified from experimental groups at late time point. 


\section{Chapter 3 - Evaluation of adenylate cyclase toxoid antigen in acellular pertussis vaccines using a}

Bordetella pertussis challenge model in mice...................................................................................87

Figures

Figure 1. RTX only immunization does not protect against $B$. pertussis challenge in CD-1 mice nor does including RTX with $1 / 5^{\text {th }}$ aP improve clearance kinetics but ACT neutralizing antibodies are produced. 113 Figure 2. Respiratory tract bacterial burden of CD-1 mice immunized with aP vaccines or aPs supplemented with RTX antigen.

Figure 3: Serological responses from mice immunized with aP titrations or aPs supplemented with RTX.

Figure 4: Analysis of proinflammatory cytokines from lungs of mice immunized with aP titrations or aPs supplemented with RTX.

Figure 5: Flow cytometric analysis of blood, pulmonary, and nasal lavage neutrophils from mice immunized with aP titrations or aPs supplemented with RTX.

Figure 6: Flow cytometric analysis of macrophages from lung and nasal lavage of mice immunized with aP titrations or aPs supplemented with RTX.

Supplemental Figures

Figure S1. RTX only nor RTX supplemented aP immunizations alter B. pertussis clearance in CD1 mice.124

Figure S2. Mouse inflammatory cytokine array of lung homogenate supernatant. .125

Figure S3. Cytokine analysis of lung homogenates of CD1 mice immunized with various aP doses and supplemented with RTX antigen.

Figure S4. Cytokine analysis of lung homogenates of CD1 mice immunized with various aP doses and supplemented with RTX antigen.

Figure S5: Serological responses from mice immunized with aP titrations or aPs supplemented with RTX.

Figure S6: Representative images and gating strategy for neutrophils

Figure S7. Heat map modeling of the effects of base aP dose and supplementation of RTX antigen on correlates of protection in mice.

Chapter 4- Intranasal acellular pertussis vaccine provides mucosal immunity and protects mice from

Figures

Figure 1: Localization of acellular pertussis vaccine in the upper and lower respiratory system after IN vaccination. 155

Figure 2: Acellular pertussis vaccine particle localization altered by curdlan adjuvant. .......................157

Figure 3: Intranasal immunization induces production of anti-PT and anti-FHA IgG in serum............... 158

Figure 4: Intranasal immunization induces production of anti-B. pertussis IgA in respiratory system.... 159

Figure 5: Intranasal immunization decreased pulmonary pro-inflammatory cytokines during challenge. 
Figure 6: Intranasal immunization reduced neutrophil and macrophage accumulation in the lung and

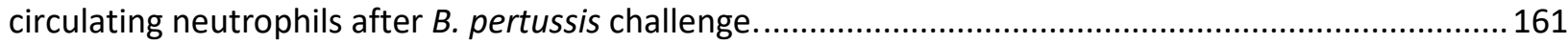

Figure 7: Intranasal immunization reduced respiratory B. pertussis bacterial burden .......................... 162

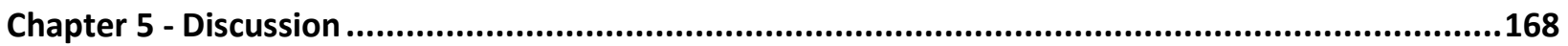

Figure 1: Proposed model of IN-caP induced protection................................................................. 177

Figure 2: Summary of proposed mucosal immune response to B. pertussis. ........................................ 181 


\section{List of Tables}

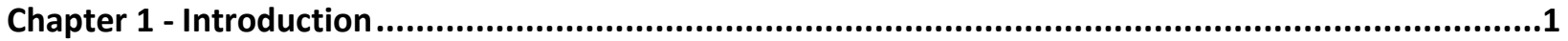

Table 1: Immune response to B. pertussis following previous infection or vaccination.......................... 18

Chapter $\mathbf{2}$ - Characterizing the innate and adaptive responses of immunized mice to Bordetella pertussis infection using in vivo imaging and transcriptomic analysis..........................................33

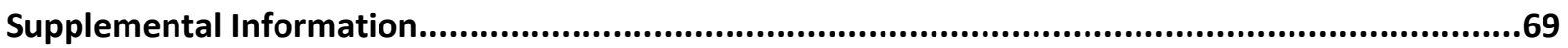

Supplementary Table 1. Compositions of vaccines of this study ......................................................... 71

Supplementary Table 2. Statistical analysis from Figure 3abc.......................................................... 72

Supplementary Table 3: Relevant statistical differences in relative fold changes of neutrophil luminescence of Bp challenged mice.

Supplementary Table 4: Relevant statistical differences between cytokine production in lungs of immunized and $B p$ challenged NECre luc mice.

Chapter 3 - Evaluation of adenylate cyclase toxoid antigen in acellular pertussis vaccines using a Bordetella pertussis challenge model in mice.

Table 1: Composition of vaccines for Fig. 1

Table 2: Composition of vaccines used in the titration of aP and RTX supplementation studies 


\section{List of Nomenclature}

1. WHO: World Health Organization

2. WP,WCV: Whole-cell pertussis vaccine

3. DTP: Diphtheria, Tetanus toxoid and whole-cell pertussis vaccine

4. DT: Diphtheria and Tetanus toxoids

5. aP, ACV, DTaP: Acellular pertussis vaccine

6. FHA: filamentous hemagglutinin

7. PT: Pertussis toxin

8. Fim: Fimbriae

9. PRN: Pertactin

10. vags: virulence associated genes

11. cAMP: cyclic adenosine monophosphate

12. LPF: leukocytosis-inducing factor

13. ACT: Adenylate cyclase toxin

14. AC: Adenylate cyclase domain

15. APC: Antigen presenting cells

16. PAMPS: Pathogen-associated molecular patterns

17. LOS: lipooligosaccharide

18. NET: Neutrophil extracellular traps

19. SIgA: Secretory immunoglobulin A

20. TRM: Tissue resident memory $T$ cells

21. IVIS: in vivo imaging systems

22. RTX: DTaP + RTX antigen

23. p.c.: post-challenge

24. IPA: Ingenuity Pathway Analysis

25. IN: Intranasal

26. IM: Intramuscular

27. IP: Intraperitoneal

28. IP-aP: Intraperitoneal administered DTaP

29. IP-wP: Intraperitoneal whole-cell pertussis vaccine

30. IP-caP: Intraperitoneal DTaP with curdlan

31. IN-aP: Intranasal administered DTaP

32. IN-caP: Intranasal administered DTaP with curdlan

33. IN-curdlan: Intranasal administered curdlan only

34. ELISA: Enzyme-linked immunosorbent assay

35. STING: Stimulator of interferon genes receptor

36. DAMPs: Damage-associated molecular patterns

37. NALT: Nasal-associated lymphoid tissue

38. $M$ cells: Microfold cells

39. DC cells: Dendritic cells 


\section{Chapter 1 - Introduction Disease Overview}

Pertussis is an exclusively human pathogen that exists in all populations worldwide. In the pre-vaccine era, the number of cases often exceeded 200,000 annually in the United States, peaking at more than 250,000 in $1934{ }^{1}$. The infection occurs in three stages: catarrhal, paroxysmal, and convalescent. In the catarrhal stage, symptoms can include a mild cough, rhinorrhea and lacrimation; similar to other upper respiratory infections ${ }^{2,3}$. Over time the cough worsens in frequency and intensity as the disease reaches the paroxysmal phase, normally ranging from 2-8 weeks ${ }^{3}$. The paroxysmal phase is defined by repeated coughing fits lasting 5-10 coughs per minute, known as a paroxysm. Following a paroxysm, an individual performs a massive inspiratory effort to refill the lungs with air resulting in the classic whoop ${ }^{4}$. The coughing fits are associated with production of high amounts of mucus and are commonly followed by post-tussive emesis ${ }^{5}$. Additional symptoms can include elevated white blood cells counts (lymphocytosis or leukocytosis), bronchopneumonia, and hypoxia. In the most severe cases these symptoms can be fatal and from $2000-20160.1 \%$ of cases resulted in fatalities, $88.8 \%$ of which occurred in infants ${ }^{6}$. The transition to the convalescent phase occurs over several weeks or months and is associated with a reduction in severity of paroxysms and other symptoms ${ }^{7}$.

Pertussis is a highly contagious respiratory disease that is spread through aerosolized B. pertussis, a dispersal method capable of infecting surrounding individuals ${ }^{8}$. Household contact studies have shown that transmission between family members is common and capable of causing mild or asymptomatic infections in adults. One study found that $46 \%$ of asymptomatic household contacts had antibody titers to $B$. pertussis 9. An aerosolized dispersal coupled with an incubation period between 7-10 days leads

to a contagious disease capable of infecting multiple individuals ${ }^{10}$. So much so, that pertussis still occurs in cyclical epidemics every 2-5 years; as it did in the pre-vaccine era ${ }^{6}$. Pertussis surveillance data shows that in United States cases rose from 1,010 in 1976, to a peak of 48,000 cases in 2012 (Figure 1) ${ }^{1}$. The increase of pertussis cases is also occurring in other countries that have adopted acellular vaccines such as the 
United Kingdom and Australia ${ }^{11}$, suggesting the cyclical pattern of pertussis epidemics is unchanged by modern vaccines.

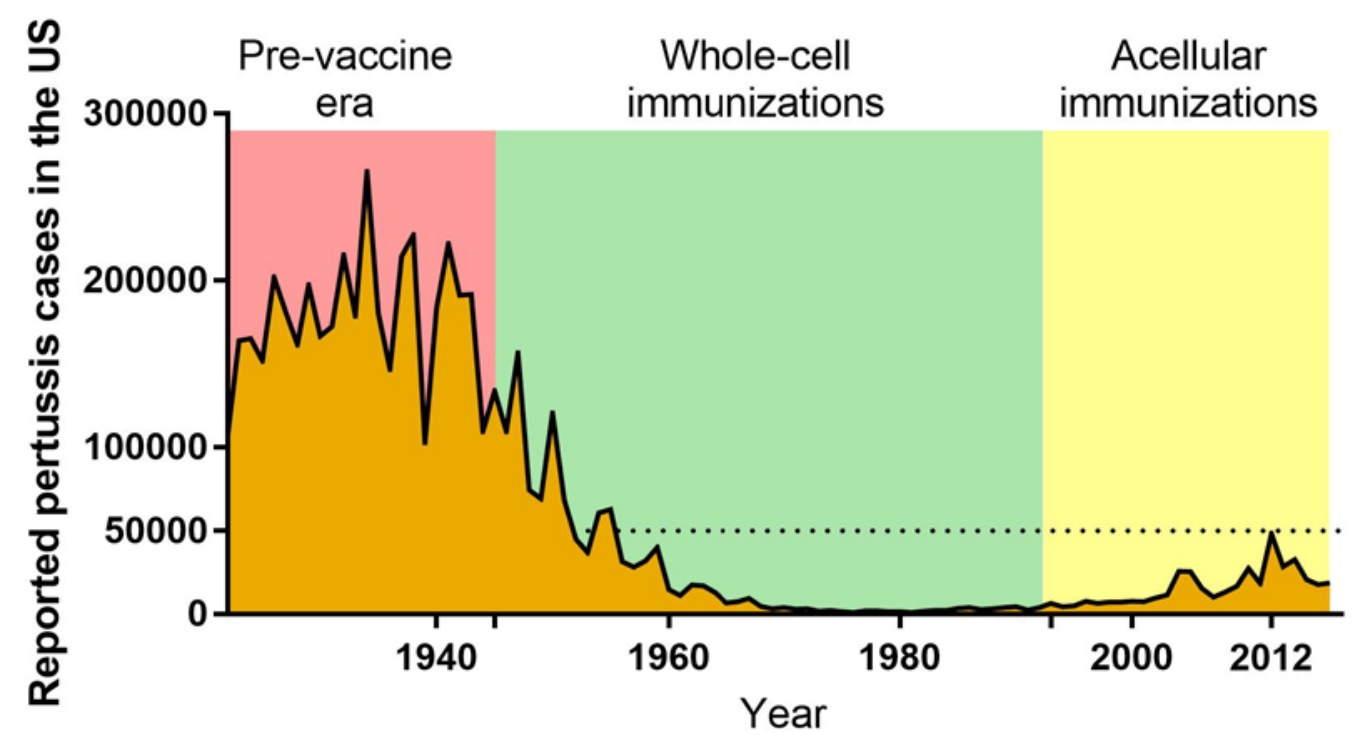

Figure 1: Incidence of clinical B. pertussis cases in the United States from 19222017. Data reported from CDC.

\section{History of B. pertussis vaccination and the increase in B. pertussis incidence}

In 1906, Jules Bordet and Octave Gengou were the first scientists to isolate the bacteria B. pertussis from an individual. Prior to the isolation of the bacteria, a disease with the same characteristics of Pertussis had been described since the middle ages ${ }^{12}$. The first documented outbreak occurred in Paris during $1578{ }^{12}$. Until the 1930 s there was still a debate as to whether $B$. pertussis was the causative agent of Pertussis, due to repeated failed attempts to culture the bacteria from a coughing patient ${ }^{13}$. Before the 1950 s, the World Health Organization (WHO) estimates that nearly half of all children that came into contact with $B$. pertussis would develop symptomatic pertussis ${ }^{14}$. The widespread implementation of a killed whole-cell pertussis (wP) vaccine drastically reduced the incidence and mortality of pertussis, to the point that in 1975 , it was proposed as a candidate for eradication ${ }^{15}$. However, beginning in the $1980 \mathrm{~s}$, the number of cases began to increase in countries with high vaccine coverage ${ }^{14}$. Around this time concerns arose regarding the reactogenicity of WP vaccines. In the late 1970s a landmark study examined the reactogenicity of whole-cell pertussis vaccine combined with diphtheria 
and tetanus toxoids (DTP) compared to diphtheria and tetanus toxoids alone (DT) ${ }^{16}$. The authors determined a correlation with redness, pain, and swelling at the injection site; as well as fever, and persistent crying when compared to DT immunization alone. In the early years of DTP vaccination there were reports of serious neurologic events and in some cases death ${ }^{17}$. Understandably, this condition of "vaccine encephalopathy" arose as a major concern against pertussis immunization. As a result of these concerns, neurologic symptoms following pertussis immunization were extensively studied. Initial findings suggested that a correlation existed; however, these experiments lacked proper control groups, and multiple conditions could explain the neurological symptoms. A welldesigned study, the National Childhood Encephalopathy Study, concluded there was no correlation between encephalopathy and pertussis immunization, and that cases of vaccine encephalopathy were the first seizures of infantile epilepsy ${ }^{18}$. In the following years DTP formulations were altered to be safer; however, in some cases, immunization was not as effective and resulted in some countries stopping DTP immunizations all together ${ }^{19}$. These inconsistencies in WP formulations and public concern pushed for the development of safer pertussis vaccines composed of acellular proteins with DT toxoids (DTaP) ${ }^{5}$. It is important to note that, wP pertussis vaccines are still widely used today, especially in low- to middle-income countries; where they are effective at controlling the number of cases.

The first DTaP vaccines were developed in Japan by Sato and colleagues; which consisted mainly of filamentous hemagglutinin (FHA), a membrane-bound adhesin of $B$. pertussis, with lower amounts of chemically detoxified pertussis toxin (PT), over time other adhesins were included, fimbriae (Fim) proteins and pertactin (PRN) ${ }^{20}$. As development of DTaP vaccines continued the amount of PT was increased equal to the concentration of FHA ${ }^{21}$. This generated a more specific immune response capable of neutralizing key virulence factors of $B$. pertussis while reducing fever, and local inflammatory reactions seen with wP immunizations. At the time, the Sato vaccines were the needed solution because they demonstrated low toxicity in mice, but were protective, generating high serum titers to antigens. When DTaP vaccines were moved to humans they were less reactogenic than WP immunizations, but still induced a robust antibody response ${ }^{22}$. Evidence from household-contact studies and a decrease in 
pertussis surveillance further supported the success of these DTaP vaccines, leading other countries, including the US to pursue DTaP vaccines ${ }^{23}$.

A key difference between the development of WP vaccines and DTaP vaccines was the method used for testing vaccine efficacy. After early inconsistencies in wP preparation, which resulted in ineffective vaccines, a validation method was developed to confirm lot validation ${ }^{5}$. Pearl Kendrick and others developed the mouse potency test in the mid1940s that enabled the prediction of WP vaccine efficacy ${ }^{24}$. Briefly, this used an intraperitoneal injection of a test vaccine and a standard vaccine into young mice; then mice were challenged with $10^{5}$ of live $B$. pertussis through intracerebral inoculation 14 to 17 days after the immunization. Vaccine-induced protection was determined by survival of the mouse. Vaccine potency was assessed as international units, determined by comparing mouse survival following different lots of wPs compared to survival following a standard vaccine. The problem was that DTaP vaccine-induced protection could not be fully validated using Kendrick's mouse potency test, as DTaP immunizations did not protect against lethality in the intracerebral challenge ${ }^{25}$. To address this, a modified Kendrick test was developed to assess vaccine potency of DTaP immunizations ${ }^{26}$. This protocol extended the time between immunization and challenge allowing for the generation of an antibody response ${ }^{26,27}$. This led to the validity of DTaP vaccines to be determined from antibody titers against the individual antigens composed in a particular vaccine. As opposed, to the survival of mice following intracerebral challenge in wP validation tests. However, evidence now suggests that IgG antibody titers are not a true correlate of protection against pertussis ${ }^{28}$. As a result, Guiso et al. developed an intranasal mouse challenge model, where $B$. pertussis was capable of colonizing the lung. This protocol led to the validity of DTaP vaccines based on serological titers and clearance of the pathogen from the lower-respiratory system of mice ${ }^{25}$.

In the United States vaccine coverage of DTaP has remained high and as of 2016 $93.7 \%$ of children aged $19-35$ months have received $\geq 3$ doses ${ }^{29}$. Nevertheless, a WHO review concluded that the United States, along with Australia, England, Wales and Portugal are experiencing a pertussis resurgence based on increased morbidity and hospitalizations ${ }^{14}$. The increase in incidence of pertussis cases suggests that 
current DTaP vaccines do not offer complete protection against $B$. pertussis. Multiple hypotheses have been proposed explaining the resurgence of incidence of pertussis including shortcomings of DTaP vaccines, vaccine-driven evolution, and increased surveillance of pertussis cases.

\section{Waning DTaP Vaccine}

Evidence that DTaP vaccine efficacy waned earlier than wP first arose in the early 2000s ${ }^{30}$. The mechanisms of protection between DTaP and WP vaccines will be explored at length later in this chapter. Presently, human data exists suggesting differences in the long-term protection from individuals immunized with either WP or DTaP vaccines, suggesting that WP vaccines offer longer protection ${ }^{31}$. Studies in the US and Australia examining cohorts of children receiving either wP or DTaP priming immunizations, followed by DTaP booster immunizations have allowed for the study of the different responses ${ }^{31}$. A study comparing protection up to 2 years post-booster immunization found higher IgG titers, memory B-cell numbers, and T-cell cytokine responses in DTaP primed individuals compared to WP primed children shortly after booster immunization ${ }^{32}$. At 2 years post-boost DTaP primed children IgG titers remained higher, but only a Th1 cellular response was observed in WP primed children 32. Another study used a similar experimental design; however, they examined protection in wP primed children two years later than they did in DTaP primed children 33. Their findings suggested that even with an additional 2 years post-boost WP primed children exhibited a higher cytokine response, and more CD4 and CD8 T cell proliferation to $B$. pertussis antigens ${ }^{33}$. A large case-control study in northern California found that DTaP protection waned unexpectedly fast following the fifth dose of DTaP ${ }^{34}$. A parallel study also, found that after the fifth dose of DTaP the odds of acquiring pertussis increased on average $42 \%$ each year ${ }^{35}$. This trend continued as the same group compared pertussis rates among adolescents who received the Tdap booster, which consists of a lower concentration of vaccine antigens with the addition of Fim antigen. These studies found that Tdap effectiveness dropped from $69 \%$ the first year after vaccination to an alarming $9 \%$ after 4 years ${ }^{36}$. Predictably, a study found that individuals who only received DTaP immunizations compared to those who received the WP vaccine schedule were at greater risk of contracting pertussis ${ }^{37}$. Together these 
data clearly demonstrate the waning of protection provided by DTaP vaccines following a completed vaccine schedule.

\section{Asymptomatic Transmission}

Recently, data has emerged suggesting asymptomatic transmission in humans based on five areas of research ${ }^{38}$. First, it is known that subclinical levels of pertussis exist in countries such as the United States and the United Kingdom ${ }^{1,14}$. Secondly, household contact is a well-documented mode of transmission of pertussis $5,9,23$. However, based on compiled data from household contact studies a symptomatic individual was only identified as the source of infection in $42 \%$ of cases ${ }^{11}$. Third, the process termed "cocooning" an infant (vaccinating those that will have early contact with the infant), has not been found to increase protection among infants ${ }^{39,40}$. Fourth, is the unexpected high number of cases in infants, given the high vaccination rate in countries such as the United States ${ }^{41}$. Lastly, is the persistence of the cyclical epidemics of pertussis similar to the pre-vaccine era ${ }^{14}$.

Up to this point, human data suggesting asymptomatic infection is only correlative; however, in the non-human primate model it has been shown that asymptomatic transmission following DTaP immunization can occur ${ }^{42}$. Warfel et al., demonstrate that while convalescent or WP vaccine-induced protection inhibited colonization by $B$. pertussis and blocked transmission to an unprotected animal, DTaP immunized baboons were colonized and transmitted the infection ${ }^{42}$. It is then conceivable to think that the correlations stated above by Althouse and Scarpino ${ }^{38}$ may be occurring in humans, and asymptomatic infection could be accounting for the resurgence of pertussis in countries with strictly DTaP immunization schedules.

\section{Vaccine-driven evolution of $B$. pertussis}

$B$. pertussis is often described as an organism with limited genetic variability, although recent data has revealed substantial variation in circulating strains across different countries ${ }^{43,44}$. Weigand et al. find that this variation occurs mostly in genes that encode for immunogenic proteins, such as $p t x A$, fimH, genes encoding for PT and FHA, respectively ${ }^{45}$. Furthermore, these strains quickly spread through the population, leading researchers to conclude that these mutations may be a result of vaccine-driven 
selection ${ }^{44,46}$. Sealey et al., note the increased mutation rate in antigen-encoding genes found in various DTaP vaccines, and further mention that the frequency of the mutations has increased since the widespread incorporation of DTaP vaccines ${ }^{47}$. Perhaps the most recognized mutation is the rise of PRN deficient stains, which have spread at alarming rates across the United States and Europe ${ }^{48,49}$. In the United States a 2015 study, found that of 753 clinical isolates, $85 \%$ were PRN-deficient, a staggering statistic when PRN is considered a major immunogen in current vaccine formulations ${ }^{50}$.

\section{Increased Surveillance and Diagnosis}

As mentioned earlier, cyclical outbreaks of pertussis continue to occur. In the prevaccine era, these outbreaks were associated with high fatalities, and this was common in low and high-income countries ${ }^{11,14}$. Today, these high fatality outbreaks continue to occur mainly in low-income countries without established infant immunization programs. In these countries, positive diagnosis occur from observation of classical pertussis clinical symptoms ${ }^{51}$. However, the resurgence of pertussis cases has been observed in high-income countries with well-established vaccine programs. In these countries, cases of pertussis are now diagnosed using quantitative real-time (RT)-PCR using insertion sequences allowing for the identification of Bordetella species, such as IS481, IS1001 and IS1002 52. PCR diagnosis was first implemented for pertussis testing in the late 1980s and early 1990s and has since become the standard in developed countries 52,53. It must be considered that the increased sensitivity of pertussis diagnosis is playing a role in increased pertussis case incidence. When the WHO assessed the reemergence of pertussis they did not support a global increase in pertussis cases due to the increasing global population and increased diagnosis sensitivity ${ }^{14}$. However, taking these factors into consideration they did determine a pertussis resurgence in the United States and other developed countries ${ }^{14}$.

Together these proposed hypotheses are supported by evidence that suggests that the switch to DTaP immunizations is playing a role in the resurgence of pertussis. A proposed strategy to circumvent for this resurgence of cases is a vaccine capable of inducing a Th1/Th17 mediated immune response more similar to wP vaccines. The rationale behind this strategy is that priming with a more pro-inflammatory vaccine will 
lead to the generation of an immune response more similar to the natural infection of $B$. pertussis. The hope being, that this response extends the duration of protection and leads to clearance of the pathogen from the respiratory tract.

\section{Pathogenesis of B. pertussis}

Much of the work performed in understanding the pathogenesis has been performed using animal models of $B$. pertussis infection. $B$. pertussis colonizes the respiratory tract and much of the work has focused on the establishment of colonization following inhalation of the bacteria. Aerosolized B. pertussis is transmitted through respiratory droplets ${ }^{54}$. The bacteria attach to cilia, expressed on respiratory airway epithelial cells, through bacterial adhesins, such as Fim and FHA ${ }^{55}$.

Once in the in the host, a currently unknown natural signal activates the sensor kinase (BvgS), of the bvgAS locus, a two-component regulatory system responsible for many of the virulence factors of $B$. pertussis. The activation of the histidine kinase BvgS and the phosphorylation of the response regulator BvgA results in the transcriptional regulation of BvgAS regulated genes, known as the $\mathrm{Bvg}^{+}$phase ${ }^{55}$. Virulence factors activated in the $\mathrm{Bvg}^{+}$phase are known as vags (virulence-activated genes) and include ptx (PT), cya (adenylate cyclase toxin), bteA, (type III secretion system), fha (FHA), fim2 and fim3 (Fim); and have been classically considered essential for infection ${ }^{56-59}$. After the establishment of infection $B$. pertussis produces bacterial toxins that either enter the bloodstream or effect surrounding cells.

\section{Pertussis toxin}

Pertussis toxin (PT) is an $A B_{5}$ toxin, that is exclusively expressed by $B$. pertussis. In earlier stages of the infection, PT plays a role in adherence to ciliated respiratory cells 60. In later stages, PT inhibits chemotaxis of phagocytic cells to the site of inflammation, where it is believed to play a critical role in the clinical symptoms of pertussis ${ }^{61}$. PT binds to glycoproteins on the cell surface of a wide variety of host cells, and the entire holotoxin is phagocytosed. In the endoplasmic reticulum, the S1 subunit is released into the cytoplasm, where it catalyzes ADP-ribosylation of Gi proteins, holding them in their inactive state ${ }^{62-64}$. This results in 1) cytoplasmic increases of cyclic adenosine monophoshate (CAMP), and 2) a dysregulation of $G_{i}$ protein-coupled signaling 
pathways; PT is specifically known to inhibit macrophage and neutrophil recruitment to the site of infection ${ }^{65,66}$. In early studies by Morse and associates, PT was termed, leukocytosis-inducing factor (LPF), where they determined LPF was responsible for the increase in leukocytosis and lymphocytosis following intravenous administration of the toxin ${ }^{67}$. It was not until the work of Margaret Pittman demonstrated that LPF was an essential toxin for the pathogenesis of $B$. pertussis was the toxin referred to as $\mathrm{PT}{ }^{5,68}$. It has been shown in numerous studies that neutralization of PT alone results in vaccineinduced protection. Therefore, detoxified toxin is included in all acellular vaccines ${ }^{60,69-}$ 72.
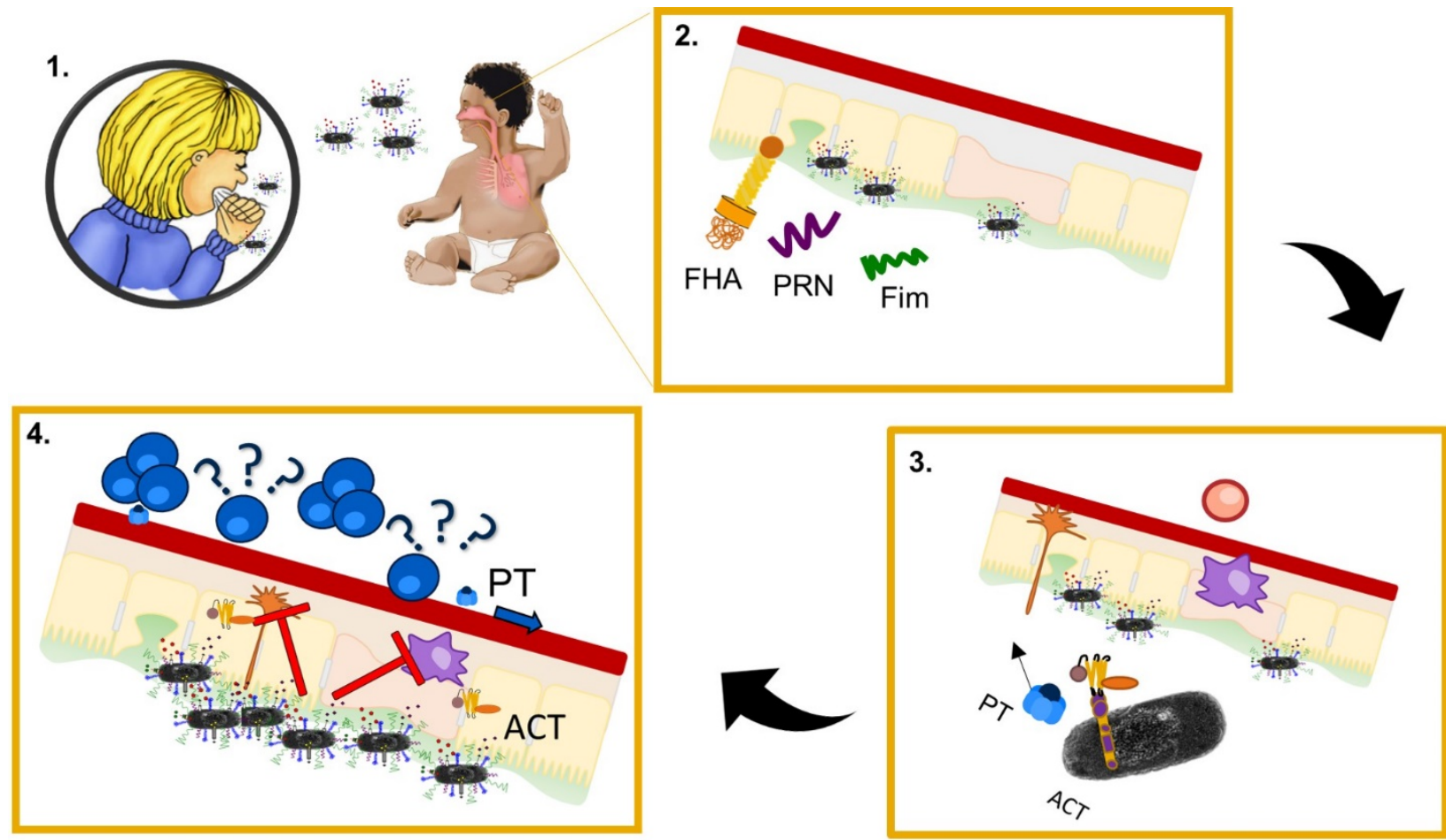

Figure 2: Pathogenesis of B. pertussis.

1) Aerosolized B. pertussis contained in expelled droplets is inhaled by individual. 2) Infection is initiated by adherence of bacteria to cilia, present on airway epithelial cells by bacteria adhesins; FHA, PRN, and Fim. 3) Activation of BvgAS phosphorelay, leads to expression of vags critical for infection, such as PT and ACT. 4) Secretion of PT and ACT dysregulate the innate immune response. PT suppresses systemic signaling of $G_{i}$ protein-coupled signaling pathways utilized by chemokine receptors, resulting in leukocytosis. ACT acts locally at the site of infection, through catalytic activity of adenylate cyclase domain and hemolysin activity of RTX domain on APCs. The immunomodulatory activity of PT, ACT along with other produced by $B$. pertussis allow for evasion of the innate immune response and persistence of infection. 


\section{Adenylate cyclase toxin}

Adenylate cyclase toxin (ACT, CyaA) is a large $177 \mathrm{kDa}$ monomeric protein, that consists of five regions: 1) N-terminal adenylate cyclase domain (AC), 2) a central hydrophobic domain, 3) a modification region responsible for acylation of the protein, 4), a repeat in toxin (RTX) hemolysin binding domain, and 5) a C-terminal secretion signal 61,73-76. ACT is secreted through a type I secretion system, through a channel formed by the CyaBDE proteins ${ }^{77}$. Following secretion, ACT is modified by acylation at sites K860 and K983.

This process is essential for toxin activity and it has been shown that acylation enhances binding to complement receptor 3 (CR3, aM 3 integrin, CD11b/CD18) ${ }^{78}$. A recent hypothesis proposes that FHA may play a role in the delivery of ACT by binding to newly secreted ACT; thereby, retaining the toxin on the surface of bacteria ${ }^{79}$. This hypothesis is supported by findings that suggest delivery of ACT requires a close association of live bacteria with target host cells ${ }^{80}$. Additionally, activation of the toxin also requires calcium, which binds in the RTX motifs of the binding domain, forming beta rolls ${ }^{81}$. Upon, this activation the $\mathrm{RTX}$ region can then bind $\mathrm{CR} 3$, leading to the penetration of host cell membrane by the hydrophobic region of ACT ${ }^{82}$. This allows for the translocation of the $A C$ domain into the cytoplasm of the host cell ${ }^{83}$. Calmodulin present in the cytoplasm of the host cell increases the catalytic activity of ACT, which drastically increases cellular cAMP to supraphysiological levels ${ }^{84}$. The increases in CAMP caused by AC activity of ACT has been shown to inhibit activation of antigenpresenting cells (APCs), as well as induce apoptosis ${ }^{84-86}$. Thus, ACT, along with PT has significant immunomodulatory properties, leading to the classification of these two toxins as the two most important virulence factors of $B$. pertussis pathogenesis ${ }^{61}$. Despite the immunomodulatory properties of $A C T$, it has yet to be included in newer generation pertussis vaccines. Limited studies have evaluated ACT as a potential antigen. Guiso et al. demonstrated purified ACT was protective in a murine intranasal challenge against $B$. pertussis ${ }^{87}$. Furthermore, the same group demonstrated anti-ACT antibodies were capable of neutralizing ACT and inhibiting ACT activity on neutrophils 88. These studies yielded encouraging results; however, after the widespread 
incorporation of DTaP into vaccine schedules progress on Pertussis vaccine research had slowed. Yet, ACT remains a strong antigen candidate for future pertussis vaccine development.

\section{Adhesin factors of $B$. pertussis}

FHA is a large, 232-kDa adhesin produced by all Bordetella spp. Studies suggest that the protein has multiple binding sites, which facilitate binding of $B$. pertussis to monocytes, macrophages, ciliated respiratory cells, and nonciliated epithelial cells ${ }^{89-91}$. FHA has been shown to be a strong immunogen and serum antibodies to FHA have been found in patients following natural infection and after immunization ${ }^{92}$. Due to the protein's immunogenicity and role in attachment, it is considered a major virulence factor and included in most DTaP vaccines.

Another group of antigens included in current 5-component DTaP formulations is the Fim proteins. Fim proteins are filamentous, polymeric cell surface proteins, that bind host cells. They are also expressed during the $\mathrm{Bvg}^{+}$growth phase, and like other vags have been shown to play a critical role in $B$. pertussis pathogenesis and adhesion to host cells ${ }^{93}$. In vivo studies using Fim-negative strains have demonstrated reduced growth in the trachea and nasopharynx of mice ${ }^{94}$, and reduced bacterial adherence in vitro ${ }^{95}$. Two serotypes of fimbriae are most commonly observed in clinical isolates, Fim2 and Fim3, and are expressed in a serotype-specific manner ${ }^{96}$. It has been demonstrated that Fim proteins are involved in bacterial agglutination, and included in current DTaP formulations as agglutinogens ${ }^{97}$.

The continued research of $B$. pertussis pathogenesis has driven antigen selection for current acellular vaccines. As the field pushes for the development of the next generation of pertussis vaccines there is evidence that other virulence factors of $B$. pertussis have the potential for inclusion into new vaccines.

\section{Immunity to B. pertussis}

Pertussis cases are most severe in infants and can even be fatal. However, the majority of individuals are able to clear the infection. Studies using animal models have suggested that cells of the innate immune response are able to control the early infection in naïve animals ${ }^{98}$. The innate immune response generated following 
challenge with $B$. pertussis has been shown to consists of antimicrobial peptides, components of the complement system, and effector cells such as macrophages, neutrophils, natural killer cells, and dendritic cells ${ }^{99}$. However, complete clearance requires the development of an adaptive, B. pertussis-specific response. Immune subversion of the innate immune response and persistence of $B$. pertussis infection are capable of delaying the generation of an adaptive immune response, suggesting that the interface between the innate and adaptive immune responses is crucial to controlling infection.

In the natural immune response, adherent bacteria colonize the respiratory epithelium. B. pertussis virulence factors act as pathogen-associated molecular patterns (PAMPS) such as: lipooligosacharide (LOS), ACT, FHA, PT. These PAMPS bind pattern recognition receptors present on mucosal epithelial cells, macrophages and dendritic cells (DCs) leading to a cascade of pro-inflammatory responses ${ }^{100,101}$. Cytokines such as: interleukin-6 (IL-6), interleukin-1 $\beta$ (IL-1 $\beta$ ), interleukin-12 (IL-12), tumor necrosis factor alpha (TNF- $\alpha$ ) and interleukin-17 (IL-17) have been shown to be produced early in B. pertussis infection and lead to recruitment of macrophages, neutrophils, DCs, and natural killer T cells to the respiratory tract ${ }^{69,98,102-104}$.

Macrophages are long-lived phagocytic cells capable of residing in the tissue. Thus, they are one of the first cell types that play a role in recognition of $B$. pertussis. In animal studies it has been shown that upon challenge with $B$. pertussis a large percentage of the bacteria are cleared early in infection, suggesting that macrophages have a role in clearance of the pathogen ${ }^{65,105}$. Studies in the mouse model have highlighted the role of macrophages in early $B$. pertussis infection, by showing that depletion of airway macrophages enhanced the infection ${ }^{65}$. It was previously mentioned that macrophages are susceptible to toxins, PT and ACT. There is evidence that some $B$. pertussis survives phagocytosis and can persist in macrophages. This suggests that dysregulation caused by bacterial toxins could lead to an intracellular niche and persistent infection ${ }^{106-108}$. Thus, there is potential that neutralization of both toxins would be a beneficial property of future pertussis vaccines. 
Neutrophils have been shown to have an interesting role in the clearance of $B$. pertussis. In the mouse model following natural infection by the bacteria, neutrophils were shown to not play an essential role in bacterial clearance ${ }^{109}$. However, when bacteria are opsonized, neutrophils do play a significant role in bacterial clearance ${ }^{109}$. Furthermore, the formation of neutrophil extracellular traps (NETs) has been observed following infection with $B$. pertussis ${ }^{110}$. Like macrophages, neutrophils as well are affected by $B$. pertussis toxins through inhibition of chemotaxis, phagocytosis, NET formation from ADP-ribosylation and CAMP accumulation ${ }^{111}$. The role of neutrophils in pertussis has not been fully explored and provides an interesting avenue for future research.

\section{Adaptive immune response}

The generation of an adaptive immune response is necessary for complete protection against $B$. pertussis. DCs from the infected respiratory tract migrate to lymph nodes to present antigen to naïve $T$ cells. The lungs of naïve adult mice do not contain high numbers of $T$ cells; however, following infection of $B$. pertussis $T$ cells are recruited to the lung which correlates with bacterial clearance from the tissue ${ }^{101}$. A critical role of $\mathrm{CD}^{+} \mathrm{T}$ cells in pertussis immunity was shown using athymic nu/nu mice, as challenge resulted in persistent infection or death ${ }^{112}$. Adoptive transfer of convalescent splenic $T$ cells into these athymic mice cleared the infection. Conversely, similar experiments using transfer of $B$. pertussis-specific $C D 8^{+} T$ cells to sublethally irradiated BALB/c mice did not result in clearance of infection, suggesting cellular responses mediated by $\mathrm{CD}^{+}$ $T$ cells play an important role in immunity to $B$. pertussis ${ }^{112}$. In particular, $\mathrm{CD} 4^{+} \mathrm{T}$ cells from infected mice produce IFN- $\gamma$ and IL-17, and not Th2 associated cytokines ${ }^{113}$. Furthermore, IFN- $\gamma^{-1}$ mice have a significantly reduced ability to clear the pathogen, and lethal disseminating $B$. pertussis infections have also been observed ${ }^{113,114}$. Studies using IL-17A ${ }^{-/}$mice, also reached similar findings, determining greater bacterial burden, and decreased neutrophil concentrations in the lungs of infected knockout mice compared to WT mice ${ }^{115}$. Data from baboons infected with $B$. pertussis correlate with data from mouse model as both models generate Th1 and Th17 immune responses following infection and colonization of $B$. pertussis ${ }^{116}$. Taken together, data from mouse 
and baboon models demonstrate that the natural infection of $B$. pertussis is cleared through the induction of a mixed Th1 and Th17 immune response ${ }^{116}$.

\section{Mucosal immune response}

$B$. pertussis is strictly a pathogen of the respiratory system. In humans all but the most severe cases occur in the upper respiratory tract ${ }^{5}$. In the mouse model, B. pertussis infection also remains in the respiratory tract and only in immunocompromised models or neonatal mice has dissemination into other organs been documented 114,117. Yet today, all commercially available pertussis vaccines are given parenterally and have not been observed to induce local immune responses in the respiratory system. Therefore, it seems that this under-explored avenue of immunity could play an important role against $B$. pertussis. Findings from the non-human primate pertussis model have strengthened this possibility. As mentioned early, Warfel et al., demonstrate DTaP immunization in baboons did not inhibit transmission of $B$. pertussis to another animal 118. While WP immunization inhibited transmission, B. pertussis colonization persisted for more than 19 days. Only in baboons that had been previously challenged with $B$. pertussis and convalescent at the time of challenge, was colonization inhibited. In another study by the same group, a strong IL-17 response was measured in nasopharyngeal wash fluid following $B$. pertussis infection ${ }^{116}$. Upon, re-challenge of convalescent baboons Th-17 associated cytokines were increased early in infection. The strength of the baboon work comes from relevance to $B$. pertussis pathogenesis in humans. However, non-human primate models are unobtainable for most researchers. Mice share similar $\mathrm{T}$ cell responses to humans and through the mouse model protection from the mucosal immune response can be currently addressed.

\section{Immunoglobulin A in the respiratory tract}

The induction of a mucosal immune response is associated with production of IgA antibody. In the mucosal surfaces IgA is the predominate isotype, where it exists in the dimeric form as secretory IgA (SIgA). Mucosal IgAs are known to block adhesion of bacteria and neutralize toxins, and serum $\lg A$ can facilitate receptor-mediated phagocytosis in other bacterial respiratory infections ${ }^{119}$. In a study using $\lg \mathrm{A}^{-/-}$mice, it was shown that upon challenge with $B$. pertussis infected mice did not exhibit a 
statistically different bacterial burden compared to WT ${ }^{120}$. More so, upon secondary challenge there was still no difference, suggesting that IgA did not play a critical role in protection in a natural infection or a primed response. Interestingly, IgA antibodies did appear to play a significant role in protection against, the closely related, Bordetella bronchiseptica clearance in the upper-respiratory tract; however, not in the lowerrespiratory tract ${ }^{120}$. The authors do state that this finding may be due to $B$. pertussis infecting human hosts, while $B$. bronchiseptica effects mainly other mammals. However, recent data has determined contradicting findings ${ }^{121}$. SlgA antibodies are transported across the mucosa through the polymeric Ig receptor. Using a mouse model deficient in this receptor, the specific role of SIgA in the respiratory tract was determined in the aforementioned study. Solans et. al. found that following intranasal administration of a live-attenuated B. pertussis vaccine, BPZE1, that in SIgA-deficient mice protection was strongly diminished compared to WT mice ${ }^{121}$. Additionally, the authors demonstrate that IL-17 plays a critical role in the production of nasal SIgA following BPZE1 immunization, as IL17\% mice produced only background levels of the antibody. Furthermore, in these mice the vaccine did not inhibit colonization. In human data, infection has been shown to be correlated with $B$. pertussis-specific IgA in the nasal secretions of infected patients ${ }^{122}$. It was found that these antibodies were detected at 23 weeks after infection, but the response is reduced to low levels around 6 months. A

possible protective role of human IgA was shown in vitro where anti-B. pertussis IgA antibodies from serum and nasal washes of convalescent individuals were shown to inhibit adherence of $B$. pertussis to human respiratory cells ${ }^{123}$.

\section{Local cellular mucosal immune response}

In the pathogenesis of $B$. pertussis, the first cells to encounter the pathogen are those of the lung-resident innate immune cells. The process of antigen-presentation via macrophages and DCs to naïve T cells in the draining lymph nodes is well understood, as described earlier. The role of Th1 and Th17 immune responses to $B$. pertussis are also well described though, most of this data comes from peripheral T cells ${ }^{114,115,124}$. It has been shown that $T$ cells from the lung and those from the spleen respond differently to bacterial antigens, and suggests a compartmentalization between $T$ cells of periphery 
and those of the lung ${ }^{125}$. More recently, it has been shown that lung $C D 4^{+}$specific to $B$. pertussis do still secrete IFN-y and/or IL-17 112,126.

The group of Kingston Mills has identified a portion of the $\mathrm{CD}^{+} \mathrm{T}$ cells secreting IFN- $\gamma$ and IL-17 are from a population known as tissue-resident memory T cells (TRM) ${ }^{127}$. These cells exhibit classical memory $T$ cell effector markers, $\mathrm{CD}_{4} 4^{+}$and $\mathrm{CD}^{2} \mathrm{~L}^{-}$, along with expression of surface markers associated with tissue retention $\mathrm{CD}^{2} 9^{+}$and/or $\mathrm{CD}_{103^{+}}{ }^{127,128}$. An innovative method was used to confirm that these $\mathrm{T}_{\mathrm{RM}}$ cells are indeed not circulating and retained in tissue. An anti-CD45 labeled-antibody was administered intravenously prior to euthanasia, thus labeling cells that were circulating as $C D 45^{+}$, while cells retained in the tissue would not be labeled. Then using another anti-CD45 labeled-antibody with a different fluorophore, cells were labeled in single-cell suspension along with other markers, $T_{\text {RM }}$ cells that were negative for the first anti-CD45 antibody, and positive for the second were classified as cells that were resident in the tissue ${ }^{127}$. In the study described above, these TRM cells provide a protective role following the secondary infection of a mouse previously infected with $B$. pertussis. Additionally, these studies were repeated and found that these cells persisted in the lung tissue for several months following the initial infection, and then expanded after rechallenge. The finding that these mucosal resident $\mathrm{T}_{\mathrm{RM}}$ are capable of secreting IL-17 and IFN- $\gamma$, suggest that this population may play a critical role in recruitment of phagocytes during the secondary immune response. More evidence suggesting a critical role was shown when migration of $\mathrm{CD}^{+} \mathrm{T}$ cells was chemically inhibited during secondary challenge with $B$. pertussis, and the infection was still cleared due to the presence of $\mathrm{T}_{\mathrm{RMs}}$ in the lung, this was also shown using adoptive transfer with similar findings ${ }^{127}$. The findings described in this section provide strong evidence that the generation of a local mucosal immune response plays a role in the clearance of $B$. pertussis following previous challenge. However, the generation of a mucosal immune response has not been observed following parenteral immunization with DTaP vaccines 129. 


\section{Pertussis vaccine-mediated immunity}

It is clear that widespread use of WP vaccines was effective. By the 1970s the average yearly rate of pertussis cases in the United States dropped to $<1$ per 100,000 population, compared to 157 cases per 100,000 population in the $1950 \mathrm{~s}^{130}$. These vaccines, still used today, are composed of killed whole $B$. pertussis organisms. As, a result the vaccines consist of numerous potential antigens and adjuvants, creating a highly immunogenic vaccine. However, this creates a degree of reactogenicity, mostly attributed to the presence of LOS ${ }^{131}$. It has been discussed that the adverse effects of wPs caused the switch in vaccine schedules to DTaP vaccines, and that following the change pertussis cases are re-emerging. Following the resurgence of pertussis, work has focused on understanding the differences in the vaccine-induced immune response following DTaP or wP immunization. While, it is known that DTaP-induced protection wanes significantly each year, initially strong IgG serum titers are observed ${ }^{132}$. A recent study demonstrated DTaP-induced IgG levels remain higher than individuals immunized by WP until 6 years of age; however, WP protection persists longer 133. Data characterizing the $\mathrm{T}$ cell responses in children following DTaP or WP priming suggests greater differences between the immunizations. Children receiving DTaP demonstrated Th2 responses, while children receiving WP preferentially induce Th1 cells ${ }^{134,135 .}$ Interestingly, current data suggests that the $T$ cell responses induced by initial vaccine priming are imprinted. Data from human patients suggests that Tdap booster immunizations do not alter the $T$ cell responses or IgG antibody subtypes induced by WP, as these patients still produce the response elicited by the WP vaccine ${ }^{133,136}$. 
Table 1: Immune response to B. pertussis following previous infection or vaccination. Summary of current literature in mice, baboons, and humans. Figure adapted from Wilk et al. ${ }^{11}$, Kapil and Merkel ${ }^{102}$, and Brummelman et al. ${ }^{101}$.

\begin{tabular}{|c|c|c|c|}
\hline & Infection & $\begin{array}{l}\text { Pertussis Whole- } \\
\text { cell vaccine (wP) }\end{array}$ & $\begin{array}{l}\text { Pertussis acellula } \\
\text { vaccine (DTaP) }\end{array}$ \\
\hline $\begin{array}{l}\text { Immune } \\
\text { Response: }\end{array}$ & Th1/Th17 & Th1/Th17 & Th2 \\
\hline $\begin{array}{l}\text { Antibody } \\
\text { Response: }\end{array}$ & $\lg G 2 a$ & $\begin{array}{l}\lg G 2 a \\
\lg G 1\end{array}$ & $\begin{array}{l}\lg G 1 \\
\lg E\end{array}$ \\
\hline $\begin{array}{c}\text { Mucosal } \\
\text { Response: }\end{array}$ & $\begin{array}{l}T_{R M} \\
\lg A\end{array}$ & $\begin{array}{l}T_{R M} \\
\lg A\end{array}$ & None \\
\hline $\begin{array}{l}\text { Mechanism } \\
\text { of Protection: }\end{array}$ & $\begin{array}{l}\text { Robust } \\
\text { proinflammatory } \\
\text { response }\end{array}$ & $\begin{array}{l}\text { Robust } \\
\text { proinflammatory } \\
\text { response }\end{array}$ & $\begin{array}{c}\text { Toxin } \\
\text { neutralizing } \\
\text { antibodies }\end{array}$ \\
\hline $\begin{array}{l}\text { Protection } \\
\text { against } \\
\text { colonization: }\end{array}$ & High & Moderate & Low \\
\hline $\begin{array}{l}\text { Persistence } \\
\text { of Protection: }\end{array}$ & Good & Moderate & Poor \\
\hline
\end{tabular}

The vaccine-induced responses following challenge with $B$. pertussis have been well characterized in mouse models. The response to $B$. pertussis in a WP immunized mouse mirrors the response to natural infection. A strong pro-inflammatory response is elicited in response to infection, resulting in enhanced bactericidal activity of macrophages and neutrophils 102,124,137. In mice, the IgG profile following WP immunization is made up primarily of $\lg \mathrm{G} 2 \mathrm{a} / \mathrm{c}$, as well as $\lg \mathrm{A}$, and $\lg \mathrm{G} 1$ antibodies, suggesting a Th1 response, whereas the IgG antibody isotype IgG2a/c (depending on 
mouse strain) ${ }^{105,115}$. This pro-inflammatory response has been shown to be dependent upon IFN- $\gamma$, as is natural infection, wP-primed IFN- $\gamma^{-1-}$ mice could not clear a challenge with $B$. pertussis ${ }^{115,138}$. Similarly, IL-17 has been shown to be induced following WPinduced protection, and clearance of $B$. pertussis was delayed in $\mathrm{IL}-17^{-1-}$ compared to WT mice, although mice did clear the infection eventually ${ }^{115}$. Recent work by Wilk et al. has demonstrated a novel finding suggesting that clearance of $B$. pertussis from the nasal cavity is dependent upon IL-17, as the $\mathrm{T}_{\mathrm{RM}}$ population was not detected in IL-17-mice, nor could they clear the infection in the nasal cavity ${ }^{129}$. Interestingly, the $T_{R M}$ population and more so bacterial clearance in the nasal cavity only occurred following natural infection with $B$. pertussis or with intraperitoneal wP immunized mice. Clearance from the nasal cavity and the $T_{R M}$ population were not found following challenge of DTaP or Tdap primed mice.

DTaP on the other hand consists of high doses of 3-5 B. pertussis antigens mainly: PT, PRN, FHA, Fim2 and Fim3. Thus, DTaP immunizations induce a more specific response that is capable of neutralizing the symptoms of the disease; however, it does not inhibit colonization or transmission ${ }^{118}$. In mice, DTaP induces a predominantly IgG1 driven humoral immune response. It is known that in mice DTaP induces a Th2 response, but also a Th17 mediated response. This was further shown through the finding that DTaP immunized $\mathrm{IL}-4^{-/-}$mice were fully protected from infection, and the protection was mediated through IL-17. The Th17 response is known to be induced by aluminum used as an adjuvant. The induction of a Th17 response has not been shown to occur in humans or non-human primates and is specific to mice $101,115,118$. Although a Th17 immune response is observed in DTaP immunized mice, it is not sufficient to induce the TRM population that was induced with wP immunization, which may play a role in the duration of memory between the vaccine-induced responses. It is clear that DTaP-vaccine induced immunity has various shortcomings including narrow specificity, different Th-cell subtype response, and different memory T cell populations.

The goal of this body of work was to better understand and improve upon the current DTaP vaccine-induced immune response. The development of DTaP vaccines occurred at a time when an answer to the reactogenic WP vaccines was desperately needed. 
Thus, the development process occurred quickly, the duration and type of immune response elicited were not designed to be tested in the safety and efficacy trials. We aimed to characterize the DTaP vaccine-induced response through a mouse model capable of addressing vaccine efficacy beyond production of $B$. pertussis-specific IgG antibodies. In chapter 2, we characterize the neutrophil response to $B$. pertussis following immunization and challenge. Using, a neutrophil reporter mouse line, NeCre luc we observed the drastic neutrophil response following challenge of a wP-primed mouse, compared to DTaP protection and even natural infection. We used RNAseq analysis to observe transcriptional changes between the vaccinated groups to further characterize pro-inflammatory response. The work in chapter 3 , discusses the titration of DTaP immunization dose to a relevant immunization dose for murine studies. In this chapter, we aimed to determine a sub-optimal vaccine dose. Defining this dose would enable us to determine effects of potential antigens. Furthermore, we implemented an antigen of a key virulence factor of $B$. pertussis, ACT. The antigen (RTX) induced a strong serum IgG response that was capable of neutralizing the toxin in vitro. Inclusion of the antigen resulted in increased bacterial clearance and a reduction in the proinflammatory response following $B$. pertussis infection. Data from non-human primates and mice studies suggest that the lack of clearance of $B$. pertussis from the upperrespiratory in DTaP protected mice was a shortcoming of the vaccine. In chapter 4 , we address the mucosal immune response by characterizing DTaP vaccine-induced immunity when the vaccine was administered intranasally. In this work we determined that intranasal immunization with DTaP can generate a local mucosal immune response. When the vaccine was administered with a Th17 promoting adjuvant we detected a local IL-17 response at the site of infection and production of IgA in the respiratory tract. In chapter 5 , the implications of these works in the generation of future pertussis vaccines are discussed. The current state of pertussis vaccine research is at a crossroads due to the uncertainty of which vaccine avenue to pursue, this work establishes a safe, intranasal vaccine with parameters that could be used to establish protection in mice and non-human primates. 


\section{References}

1 CDC, Baldy SWRLM. Manual for the surveillance of vaccine-preventable diseases. Atlanta, GA: Centers for Disease Control and Prevention; 2015.

2 Paddock CD, Sanden GN, Cherry JD, Gal AA, Langston C, Tatti KM, et al. Pathology and Pathogenesis of Fatal Bordetella pertussis Infection in Infants. Clin Infect Dis 2008;47:328-38. https://doi.org/10.1086/589753.

3 Mattoo S, Cherry JD. Molecular pathogenesis, epidemiology, and clinical manifestations of respiratory infections due to $<i>$ Bordetella pertussis $<i />$ and other Bordetella subspecies. Clin Microbiol Rev 2005;18:326-82. https://doi.org/10.1128/CMR.18.2.326-382.2005.

4 Heininger U, Klich K, Stehr K, Cherry JD. Clinical findings in Bordetella pertussis infections: results of a prospective multicenter surveillance study. Pediatrics 1997;100:E10. https://doi.org/10.1542/peds.100.6.e10.

5 Mattoo S, Cherry JD. Molecular pathogenesis, epidemiology, and clinical manifestations of respiratory infections due to Bordetella pertussis and other Bordetella subspecies. Clin Microbiol Rev 2005;18:326-82. https://doi.org/10.1128/CMR.18.2.326-382.2005.

6 Skoff TH, Hadler S, Hariri S. The Epidemiology of Nationally Reported Pertussis in the United States, 2000-2016. Clin Infect Dis 2018;68:1634-40. https://doi.org/10.1093/cid/ciy757.

7 Harnden A, Grant C, Harrison T, Perera R, Brueggemann AB, Mayon-White R, et al. Whooping cough in school age children with persistent cough: prospective cohort study in primary care. BMJ 2006;333:174-7. https://doi.org/10.1136/bmj.38870.655405.AE.

8 Warfel JM, Beren J, Merkel TJ. Airborne transmission of bordetella pertussis. $\mathrm{J}$ Infect Dis 2012;206:902-6. https://doi.org/10.1093/infdis/jis443.

9 Deen JL, Mink CM, Cherry JD, Christenson PD, Pineda EF, Lewis K, et al. Household Contact Study of Bordetella pertussis Infections. Clin Infect Dis 1995;21:1211-9. https://doi.org/10.1093/clinids/21.5.1211.

10 Stehr K, Cherry JD, Heininger U, Schmitt-Grohé S, uberall M, Laussucq S, et al. A comparative efficacy trial in Germany in infants who received either the Lederle/Takeda acellular pertussis component DTP (DTaP) vaccine, the Lederle whole-cell component DTP vaccine, or DT vaccine. Pediatrics 1998;101:1-11. https://doi.org/10.1542/peds.101.1.1.

11 Rohani P, Scarpino S V. Pertussis : epidemiology, immunology, and evolution. 1st ed. New York: Oxford University Press; 2019.

12 Cherry JD. Historical review of pertussis and the classical vaccine. J Infect Dis 1996;174 Suppl 3:S259-63. 
https://doi.org/10.1093/infdis/174.supplement_3.s259.

13 Macdonald H, Macdonald EJ. Experimental Pertussis. J Infect Dis 1933;53:32830. https://doi.org/10.1093/infdis/53.3.328.

14 Organization WH. Pertussis Vaccines: WHO position paper-August 2015 2018:145-68.

15 Kendrick PL. Can Whooping Cough Be Eradicated? J Infect Dis 1975;132:70712. https://doi.org/10.1093/infdis/132.6.707.

16 Cody CL, Baraff LJ, Cherry JD, Marcy SM, Manclark CR. Nature and Rates of Adverse Reactions Associated with DTP and DT Immunizations in Infants and Children. Pediatrics 1981;68:.

17 BYERS RK, MOLL FC. ENCEPHALOPATHIES FOLLOWING PROPHYLACTIC PERTUSSIS VACCINE. Pediatrics 1948;1:

18 Miller DL, Ross EM, Alderslade R, Bellman MH, Rawson NS. Pertussis immunisation and serious acute neurological illness in children. Br Med J (Clin Res Ed) 1981;282:1595-9. https://doi.org/10.1136/bmj.282.6276.1595.

19 Romanus V, Jonsell R, Bergquist SO. Pertussis in Sweden after the cessation of general immunization in 1979. Pediatr Infect Dis J 1987;6:364-71.

20 Sato Y, Kimura M, Fukumi H. Development of a pertussis component vaccine in Japan. Lancet (London, England) 1984;1:122-6.

21 Sato H, Sato Y. Protective antigens of Bordetella pertussis mouse-protection test against intracerebral and aerosol challenge of B. pertussis. Dev Biol Stand 1985;61:461-7.

22 Kimura M. Japanese clinical experiences with acellular pertussis vaccines. Dev Biol Stand 1991;73:5-9.

23 Noble GR, Bernier RH, Esber EC, Hardegree MC, Hinman AR, Klein D, et al. Acellular and whole-cell pertussis vaccines in Japan. Report of a visit by US scientists. JAMA 1987;257:1351-6.

24 Kendrick PL, Eldering G, Dixon MK, Misner J. Mouse Protection Tests in the Study of Pertussis Vaccine: A Comparative Series Using the Intracerebral Route for Challenge. Am J Public Heal Nations Heal 1947;37:803-10.

25 Guiso N, Capiau C, Carletti G, Poolman J, Hauser P. Intranasal murine model of Bordetella pertussis infection. I. Prediction of protection in human infants by acellular vaccines. Vaccine 1999;17:2366-76. https://doi.org/10.1016/S0264410X(99)00037-7.

26 Xing D, Gaines Das R, Douglas-Bardsley A, Asokanathan C, Corbel M. An international collaborative study of the effect of active pertussis toxin on the modified Kendrick test for acellular pertussis vaccines. Biologicals 2014;42:101-8. https://doi.org/10.1016/j.biologicals.2013.11.008. 
27 Xing D, Markey K, Gaines Das R, Feavers I. Whole-cell pertussis vaccine potency assays: the Kendrick test and alternative assays. Expert Rev Vaccines 2014;13:1175-82. https://doi.org/10.1586/14760584.2014.939636.

28 Cherry JD, Gornbein J, Heininger U, Stehr K. A search for serologic correlates of immunity to Bordetella pertussis cough illnesses. Vaccine 1998;16:1901-6.

29 Hill Elam-Evans LD, Yankey D, Singleton JA, Kang Y. HA. Vaccination Coverage Among Children Aged 19-35 Months - United States, 2017. MMWR Morb Mortal Wkly Rep 2018 2017:1123-1128.

https://doi.org/http://dx.doi.org/10.15585/mmwr.mm6740a4External.

30 Lacombe K, Yam A, Simondon K, Pinchinat S, Simondon F. Risk factors for acellular and whole-cell pertussis vaccine failure in Senegalese children. Vaccine 2004;23:623-8. https://doi.org/10.1016/j.vaccine.2004.07.007.

31 Sheridan SL, Frith K, Snelling TL, Grimwood K, McIntyre PB, Lambert SB. Waning vaccine immunity in teenagers primed with whole cell and acellular pertussis vaccine: recent epidemiology. Expert Rev Vaccines 2014;13:1081-106. https://doi.org/10.1586/14760584.2014.944167.

32 Schure R-M, Hendrikx LH, de Rond LGH, Öztürk K, Sanders EAM, Berbers GAM, et al. Differential T- and B-Cell Responses to Pertussis in Acellular VaccinePrimed versus Whole-Cell Vaccine-Primed Children 2 Years after Preschool Acellular Booster Vaccination. Clin Vaccine Immunol 2013;20:1388-95. https://doi.org/10.1128/CVI.00270-13.

33 Smits K, Pottier G, Smet J, Dirix V, Vermeulen F, De Schutter I, et al. Different T cell memory in preadolescents after whole-cell or acellular pertussis vaccination. Vaccine 2013;32:111-8. https://doi.org/10.1016/j.vaccine.2013.10.056.

34 Witt MA, Katz PH, Witt DJ. Unexpectedly Limited Durability of Immunity Following Acellular Pertussis Vaccination in Preadolescents in a North American Outbreak. Clin Infect Dis 2012;54:1730-5. https://doi.org/10.1093/cid/cis287.

35 Klein NP, Bartlett J, Rowhani-Rahbar A, Fireman B, Baxter R. Waning Protection after Fifth Dose of Acellular Pertussis Vaccine in Children. N Engl J Med 2012;367:1012-9. https://doi.org/10.1056/NEJMoa1200850.

36 Klein NP, Bartlett J, Fireman B, Baxter R. Waning Tdap Effectiveness in Adolescents. Pediatrics 2016;137:e20153326-e20153326. https://doi.org/10.1542/peds.2015-3326.

37 Witt MA, Arias L, Katz PH, Truong ET, Witt DJ. Reduced Risk of Pertussis Among Persons Ever Vaccinated With Whole Cell Pertussis Vaccine Compared to Recipients of Acellular Pertussis Vaccines in a Large US Cohort. Clin Infect Dis 2013;56:1248-54. https://doi.org/10.1093/cid/cit046.

38 Althouse BM, Scarpino S V. Asymptomatic transmission and the resurgence of Bordetella pertussis. BMC Med 2015;13:146. https://doi.org/10.1186/s12916-0150382-8. 
39 Healy CM, Rench MA, Wootton SH, Castagnini LA. Evaluation of the Impact of a Pertussis Cocooning Program on Infant Pertussis Infection. Pediatr Infect Dis J 2015;34:22-6. https://doi.org/10.1097/INF.0000000000000486.

40 Carcione D, Regan AK, Tracey L, Mak DB, Gibbs R, Dowse GK, et al. The impact of parental postpartum pertussis vaccination on infection in infants: A populationbased study of cocooning in Western Australia. Vaccine 2015;33:5654-61. https://doi.org/10.1016/j.vaccine.2015.08.066.

41 Masseria C, Martin CK, Krishnarajah G, Becker LK, Buikema A, Tan TQ. Incidence and Burden of Pertussis Among Infants Less Than 1 Year of Age. Pediatr Infect Dis J 2017;36:e54-61. https://doi.org/10.1097/INF.0000000000001440.

42 Warfel JM, Zimmerman LI, Merkel TJ. Acellular pertussis vaccines protect against disease but fail to prevent infection and transmission in a nonhuman primate model. Proc Natl Acad Sci 2014;111:787-92. https://doi.org/10.1073/pnas.1314688110.

43 Mooi FR. Bordetella pertussis and vaccination: The persistence of a genetically monomorphic pathogen. Infect Genet Evol 2010;10:36-49. https://doi.org/10.1016/j.meegid.2009.10.007.

44 Bart MJ, Harris SR, Advani A, Arakawa Y, Bottero D, Bouchez V, et al. Global Population Structure and Evolution of Bordetella pertussis and Their Relationship with Vaccination. MBio 2014;5:e01074. https://doi.org/10.1128/mBio.01074-14.

45 Weigand MR, Peng Y, Loparev V, Batra D, Bowden KE, Burroughs M, et al. The History of Bordetella pertussis Genome Evolution Includes Structural Rearrangement. J Bacteriol 2017;199:e00806-16. https://doi.org/10.1128/JB.00806-16.

46 Octavia S, Maharjan RP, Sintchenko V, Stevenson G, Reeves PR, Gilbert GL, et al. Insight into Evolution of Bordetella pertussis from Comparative Genomic Analysis: Evidence of Vaccine-Driven Selection. Mol Biol Evol 2011;28:707-15. https://doi.org/10.1093/molbev/msq245.

47 Sealey KL, Belcher T, Preston A. Bordetella pertussis epidemiology and evolution in the light of pertussis resurgence. Infect Genet Evol 2016;40:136-43. https://doi.org/10.1016/j.meegid.2016.02.032.

48 Pawloski LC, Queenan AM, Cassiday PK, Lynch AS, Harrison MJ, Shang W, et al. Prevalence and molecular characterization of pertactin-deficient Bordetella pertussis in the United States. Clin Vaccine Immunol 2014;21:119-25. https://doi.org/10.1128/CVI.00717-13.

49 Barkoff A-M, Mertsola J, Pierard D, Dalby T, Hoegh SV, Guillot S, et al. Pertactindeficient Bordetella pertussis isolates: evidence of increased circulation in Europe, 1998 to 2015. Euro Surveill 2019;24:. https://doi.org/10.2807/15607917.ES.2019.24.7.1700832. 
50 Martin SW, Pawloski L, Williams M, Weening K, DeBolt C, Qin X, et al. PertactinNegative Bordetella pertussis Strains: Evidence for a Possible Selective Advantage. Clin Infect Dis 2015;60:223-7. https://doi.org/10.1093/cid/ciu788.

51 Datta SS, Toikilik S, Ropa B, Chidlow G, Lagani W. Pertussis outbreak in Papua New Guinea: the challenges of response in a remote geo-topographical setting. West Pacific Surveill Response J 2012;3:3-6. https://doi.org/10.5365/wpsar.2012.3.3.008.

52 Templeton KE, Scheltinga SA, van der Zee A, Diederen BMW, van Kruijssen AM, Goossens $\mathrm{H}$, et al. Evaluation of real-time PCR for detection of and discrimination between Bordetella pertussis, Bordetella parapertussis, and Bordetella holmesii for clinical diagnosis. J Clin Microbiol 2003;41:4121-6. https://doi.org/10.1128/jcm.41.9.4121-4126.2003.

53 Glare EM, Paton JC, Premier RR, Lawrence AJ, Nisbet IT. Analysis of a repetitive DNA sequence from Bordetella pertussis and its application to the diagnosis of pertussis using the polymerase chain reaction. J Clin Microbiol 1990;28:1982-7.

54 Pinto M V., Merkel TJ. Pertussis disease and transmission and host responses: insights from the baboon model of pertussis. $J$ Infect 2017;74:S114-9. https://doi.org/10.1016/S0163-4453(17)30201-3.

55 Melvin JA, Scheller E V., Miller JF, Cotter PA. Bordetella pertussis pathogenesis: Current and future challenges. Nat Rev Microbiol 2014;12:274-88. https://doi.org/10.1038/nrmicro3235.

56 Merkel TJ, Stibitz S, Keith JM, Leef M, Shahin R. Contribution of regulation by the bvg locus to respiratory infection of mice by Bordetella pertussis. Infect Immun 1998;66:4367-73.

57 Scarlato V, Aricò B, Prugnola A, Rappuoli R. Sequential activation and environmental regulation of virulence genes in Bordetella pertussis . EMBO J 1991;10:3971-5.

58 Hegerle N, Rayat L, Dore G, Zidane N, Bedouelle H, Guiso N. In-vitro and in-vivo analysis of the production of the Bordetella type three secretion system effector $A$ in Bordetella pertussis , Bordetella parapertussis and Bordetella bronchiseptica. Microbes Infect 2013;15:399-408. https://doi.org/10.1016/j.micinf.2013.02.006.

59 Moon K, Bonocora RP, Kim DD, Chen Q, Wade JT, Stibitz S, et al. The BvgAS Regulon of Bordetella pertussis . MBio 2017;8:. https://doi.org/10.1128/mBio.01526-17.

60 Carbonetti NH, Artamonova G V, Mays RM, Worthington ZE V. Pertussis toxin plays an early role in respiratory tract colonization by Bordetella pertussis . Infect Immun 2003;71:6358-66. https://doi.org/10.1128/iai.71.11.6358-6366.2003.

61 Carbonetti NH. Pertussis toxin and adenylate cyclase toxin: Key virulence factors of Bordetella pertussis and cell biology tools. Future Microbiol 2010;5:455-69. https://doi.org/10.2217/fmb.09.133. 
62 Armstrong GD, Howard LA, Peppler MS. Use of glycosyltransferases to restore pertussis toxin receptor activity to asialoagalactofetuin. $\mathrm{J}$ Biol Chem 1988;263:8677-84.

63 Hazes B, Boodhoo A, Cockle SA, Read RJ. Crystal structure of the pertussis toxin-ATP complex: a molecular sensor. J Mol Biol 1996;258:661-71. https://doi.org/10.1006/jmbi.1996.0277.

64 Katada T, Tamura M, Ui M. The A protomer of islet-activating protein, pertussis toxin, as an active peptide catalyzing ADP-ribosylation of a membrane protein. Arch Biochem Biophys 1983;224:290-8.

65 Carbonetti NH, Artamonova G V, Van Rooijen N, Ayala VI. Pertussis toxin targets airway macrophages to promote Bordetella pertussis infection of the respiratory tract. Infect Immun 2007;75:1713-20. https://doi.org/10.1128/IAI.01578-06.

66 Andreasen C, Carbonetti NH. Role of neutrophils in response to Bordetella pertussis infection in mice. Infect Immun 2009;77:1182-8. https://doi.org/10.1128/IAI.01150-08.

67 Morse SI, Morse JH. Isolation and properties of the leukocytosis- and lymphocytosis- promoting factor of Bordetella pertussis . J Exp Med 1976;143:1483-502. https://doi.org/10.1084/jem.143.6.1483.

68 Pittman M. History of the development of pertussis vaccine. Dev Biol Stand 1991;73:13-29.

69 Skarlupka AL, Linz B, Maynard JA, Harvill ET. Basics of pertussis pathogenesis. In: Rohani P, Scarpino S V, editors. Pertussis Epidemiol. immunuology, Evol. 1st ed. New York: Oxford University Press; 2019. p. 26-41.

70 Kapil P, Papin JF, Wolf RF, Zimmerman LI, Wagner LD, Merkel TJ. Maternal Pertussis Vaccination • JID 2018:217 (15 April) • 1231 The Journal of Infectious Diseases n.d. https://doi.org/10.1093/infdis/jiy022.

71 Carbonetti NH. Contribution of pertussis toxin to the pathogenesis of pertussis disease. Pathog Dis 2015;73:ftv073. https://doi.org/10.1093/femspd/ftv073.

72 Carbonetti NH, Artamonova G V., Andreasen C, Bushar N. Pertussis toxin and adenylate cyclase toxin provide a one-two punch for establishment of Bordetella pertussis infection of the respiratory tract. Infect Immun 2005;73:2698-703. https://doi.org/10.1128/IAI.73.5.2698-2703.2005.

73 Ladant D, Ullmann A. Bordetella pertussis adenylate cyclase: a toxin with multiple talents. Trends Microbiol 1999;7:172-6. https://doi.org/10.1016/S0966842X(99)01468-7.

74 Ehrmann IE, Gray MC, Gordon VM, Gray LS, Hewlett EL. Hemolytic activity of adenylate cyclase toxin from Bordetella pertussis . FEBS Lett 1991;278:79-83.

75 Wang X, Maynard JA. The Bordetella adenylate cyclase repeat-in-toxin (RTX) domain is immunodominant and elicits neutralizing antibodies. J Biol Chem 
2015;290:3576-91. https://doi.org/10.1074/jbc.M114.585281.

76 Sebo P, Osicka R, Masin J. Adenylate cyclase toxin-hemolysin relevance for pertussis vaccines. Expert Rev Vaccines 2014;13:1215-27. https://doi.org/10.1586/14760584.2014.944900.

77 Vojtova J, Kamanova J, Sebo P. Bordetella adenylate cyclase toxin: a swift saboteur of host defense. Curr Opin Microbiol 2006;9:69-75. https://doi.org/10.1016/j.mib.2005.12.011.

78 El-Azami-El-Idrissi M, Bauche C, Loucka J, Osicka R, Sebo P, Ladant D, et al. Interaction of Bordetella pertussis adenylate cyclase with CD11b/CD18: Role of toxin acylation and identification of the main integrin interaction domain. $\mathrm{J}$ Biol Chem 2003;278:38514-21. https://doi.org/10.1074/jbc.M304387200.

79 Nash ZM, Cotter PA. Bordetella filamentous hemagglutinin, a model for the two partner secretion pathway. Microbiol Spectr 2019;7:.

https://doi.org/10.1128/MICROBIOLSPEC.PSIB-0024-2018.

80 Gray MC, Donato GM, Jones FR, Kim T, Hewlett EL. Newly secreted adenylate cyclase toxin is responsible for intoxication of target cells by Bordetella pertussis . Mol Microbiol 2004;53:1709-19. https://doi.org/10.1111/j.13652958.2004.04227.x.

81 Bauche C, Chenal A, Knapp O, Bodenreider C, Benz R, Chaffotte A, et al. Structural and functional characterization of an essential RTX subdomain of Bordetella pertussis adenylate cyclase toxin. J Biol Chem 2006;281:16914-26. https://doi.org/10.1074/jbc.M601594200.

82 Saukkonen K, Cabellos C, Burroughs M, Prasad S, Tuomanen E. Integrinmediated localization of Bordetella pertussis within macrophages: role in pulmonary colonization. J Exp Med 1991;173:1143-9. https://doi.org/10.1084/jem.173.5.1143.

83 Vojtova-Vodolanova J, Basler M, Osicka R, Knapp O, Maier E, Cerny J, et al. Oligomerization is involved in pore formation by Bordetella adenylate cyclase toxin. FASEB J 2009;23:2831-43. https://doi.org/10.1096/fj.09-131250.

84 Hewlett EL, Donato GM, Gray MC. Macrophage cytotoxicity produced by adenylate cyclase toxin from Bordetella pertussis : more than just making cyclic AMP! Mol Microbiol 2006;59:447-59. https://doi.org/10.1111/j.13652958.2005.04958.x.

85 Skopova K, Tomalova B, Kanchev I, Rossmann P, Svedova M, Adkins I, et al. Cyclic AMP-elevating capacity of adenylate cyclase toxin-hemolysin is sufficient for lung infection but not for full virulence of Bordetella pertussis . Infect Immun 2017;85:. https://doi.org/10.1128//AI.00937-16.

86 Khelef N, Guiso N. Induction of macrophage apoptosis by Bordetella pertussis adenylate cyclase-hemolysin. FEMS Microbiol Lett 1995;134:27-32. https://doi.org/10.1111/j.1574-6968.1995.tb07909.x. 
87 Guiso N, Szatanik M, Rocancourt M. Protective activity of Bordetella adenylate cyclase-hemolysin against bacterial colonization. Microb Pathog 1991;11:423-31. https://doi.org/10.1016/0882-4010(91)90038-C.

88 Weingart CL, Mobberley-Schuman PS, Hewlett EL, Gray MC, Weiss AA. Neutralizing antibodies to adenylate cyclase toxin promote phagocytosis of Bordetella pertussis by human neutrophils. Infect Immun 2000;68:7152-5. https://doi.org/10.1128/iai.68.12.7152-7155.2000.

89 Melvin JA, Scheller E V., Noël CR, Cotter PA. New Insight into Filamentous Hemagglutinin Secretion Reveals a Role for Full-Length FhaB in Bordetella Virulence. MBio 2015;6:. https://doi.org/10.1128/mBio.01189-15.

90 Ishibashi Y, Relman DA, Nishikawa A. Invasion of human respiratory epithelial cells by Bordetella pertussis : Possible role for a filamentous hemagglutinin ArgGly-Asp sequence and a5ß1 integrin. Microb Pathog 2001;30:279-88. https://doi.org/10.1006/mpat.2001.0432.

91 Ahmed SS, Ellis RW, Rappuoli R. Technologies for Making New Vaccines. In: Plotkin SA, OrenStein WA, Offit PA, editors. Plotkin's Vaccines. Seventh. Philadelphia, PA: Elsevier; 2018. p. 1343-53.

92 Scheller E V., Cotter PA. Bordetella filamentous hemagglutinin and fimbriae: critical adhesins with unrealized vaccine potential. Pathog Dis 2015;73:ftv079. https://doi.org/10.1093/femspd/ftv079.

93 Willems R, Paul A, van der Heide HG, ter Avest AR, Mooi FR. Fimbrial phase variation in Bordetella pertussis : a novel mechanism for transcriptional regulation. EMBO J 1990;9:2803-9.

94 Geuijen CA, Willems RJ, Bongaerts M, Top J, Gielen H, Mooi FR. Role of the Bordetella pertussis minor fimbrial subunit, FimD, in colonization of the mouse respiratory tract. Infect Immun 1997;65:4222-8.

95 Guevara C, Zhang C, Gaddy JA, Iqbal J, Guerra J, Greenberg DP, et al. Highly differentiated human airway epithelial cells: a model to study host cell-parasite interactions in pertussis. Infect Dis (London, England) 2016;48:177-88. https://doi.org/10.3109/23744235.2015.1100323.

96 Meade BD, Lynn F, Reed GF, Mink CM, Romani TA, Deforest A, et al. Relationships between functional assays and enzyme immunoassays as measurements of responses to acellular and whole-cell pertussis vaccines. Pediatrics 1995;96:595-600.

97 Li ZM, Brennan MJ, David JL, Carter PH, Cowell JL, Manclark CR. Comparison of type 2 and type 6 fimbriae of Bordetella pertussis by using agglutinating monoclonal antibodies. Infect Immun 1988;56:3184-8.

98 Higgs R, Higgins SC, Ross PJ, Mills KHG. Immunity to the respiratory pathogen Bordetella pertussis . Mucosal Immunol 2012;5:485-500.

https://doi.org/10.1038/mi.2012.54. 
99 Solans L, Locht C. The Role of Mucosal Immunity in Pertussis. Front Immunol 2018;9:. https://doi.org/10.3389/FIMMU.2018.03068.

100 Nishida M, Suda R, Nagamatsu Y, Tanabe S, Onohara N, Nakaya M, et al. Pertussis Toxin Up-regulates Angiotensin Type 1 Receptors through Toll-like Receptor 4-mediated Rac Activation. J Biol Chem 2010;285:15268-77. https://doi.org/10.1074/jbc.M109.076232.

101 Brummelman J, Wilk MM, Han WGH, van Els CACM, Mills KHG. Roads to the development of improved pertussis vaccines paved by immunology. Pathog Dis 2015;73:ftv067. https://doi.org/10.1093/femspd/ftv067.

102 Kapil P, Merkel TJ. Pertussis vaccines and protective immunity. Curr Opin Immunol 2019;59:72-8. https://doi.org/https://doi.org/10.1016/j.coi.2019.03.006.

103 Gregg KA, Harberts E, Gardner FM, Pelletier MR, Cayatte C, Yu L, et al. Rationally designed TLR4 ligands for vaccine adjuvant discovery. MBio 2017;8:. https://doi.org/10.1128/mBio.00492-17.

104 CARBONETTI N. Immunomodulation in the pathogenesis of Bordetella pertussis infection and disease. Curr Opin Pharmacol 2007;7:272-8. https://doi.org/10.1016/j.coph.2006.12.004.

105 Higgs R, Higgins SC, Ross PJ, Mills KHG. Immunity to the respiratory pathogen Bordetella pertussis . Mucosal Immunol 2012;5:485-500. https://doi.org/10.1038/mi.2012.54.

106 Lamberti YA, Hayes JA, Perez Vidakovics ML, Harvill ET, Rodriguez ME. Intracellular trafficking of Bordetella pertussis in human macrophages. Infect Immun 2010;78:907-13. https://doi.org/10.1128/IAI.01031-09.

107 Lamberti Y, Gorgojo J, Massillo C, Rodriguez ME. Bordetella pertussis entry into respiratory epithelial cells and intracellular survival. Pathog Dis 2013;69:194-204. https://doi.org/10.1111/2049-632X.12072.

108 Valdez HA, Oviedo JM, Gorgojo JP, Lamberti Y, Rodriguez ME. Bordetella pertussis modulates human macrophage defense gene expression. Pathog Dis 2016;74:ftw073. https://doi.org/10.1093/femspd/ftw073.

109 Weingart CL, Weiss a a. Bordetella pertussis virulence factors affect phagocytosis by human neutrophils. Infect Immun 2000;68:1735-9. https://doi.org/10.1128/IAI.68.3.1735-1739.2000.

110 Eby JC, Gray MC, Hewlett EL. Cyclic AMP-mediated suppression of neutrophil extracellular trap formation and apoptosis by the Bordetella pertussis adenylate cyclase toxin. Infect Immun 2014;82:5256-69. https://doi.org/10.1128/IAI.0248714.

111 Eby JC, Hoffman CL, Gonyar LA, Hewlett EL. Review of the neutrophil response to Bordetella pertussis infection. Pathog Dis 2015;73:ftv081.

https://doi.org/10.1093/femspd/ftv081. 
112 Mills KH, Barnard A, Watkins J, Redhead K. Cell-mediated immunity to Bordetella pertussis : role of Th1 cells in bacterial clearance in a murine respiratory infection model. Infect Immun 1993;61:399-410.

113 Barbic J, Leef MF, Burns DL, Shahin RD. Role of gamma interferon in natural clearance of Bordetella pertussis infection. Infect Immun 1997;65:4904-8. https://doi.org/10.1371/journal.ppat.1003264.

114 Mahon BP, Sheahan BJ, Griffin F, Murphy G, Mills KH. Atypical disease after Bordetella pertussis respiratory infection of mice with targeted disruptions of interferon-gamma receptor or immunoglobulin mu chain genes. J Exp Med 1997;186:1843-51.

115 Ross PJ, Sutton CE, Higgins S, Allen AC, Walsh K, Misiak A, et al. Relative contribution of Th1 and Th17 cells in adaptive immunity to Bordetella pertussis : towards the rational design of an improved acellular pertussis vaccine. PLoS Pathog 2013;9:e1003264. https://doi.org/10.1371/journal.ppat.1003264.

116 Warfel JM, Merkel TJ. Bordetella pertussis infection induces a mucosal IL-17 response and long-lived Th17 and Th1 immune memory cells in nonhuman primates. Mucosal Immunol 2013;6:787-96. https://doi.org/10.1038/mi.2012.117.

117 Scanlon KM, Snyder YG, Skerry C, Carbonetti NH. Fatal Pertussis in the Neonatal Mouse Model Is Associated with Pertussis Toxin-Mediated Pathology beyond the Airways. Infect Immun 2017;85:. https://doi.org/10.1128/IAI.00355-17.

118 Warfel JM, Zimmerman LI, Merkel TJ. Acellular pertussis vaccines protect against disease but fail to prevent infection and transmission in a nonhuman primate model. Proc Natl Acad Sci 2014;111:787-92. https://doi.org/10.1073/pnas.1314688110.

119 Rodríguez A, Tjärnlund A, Ivanji J, Singh M, García I, Williams A, et al. Role of $\lg A$ in the defense against respiratory infections. Vaccine 2005;23:2565-72. https://doi.org/10.1016/j.vaccine.2004.11.032.

120 Wolfe DN, Kirimanjeswara GS, Goebel EM, Harvill ET. Comparative Role of Immunoglobulin A in Protective Immunity against the Bordetellae. Infect Immun 2007;75:4416. https://doi.org/10.1128/IAl.00412-07.

121 Solans L, Debrie A-S, Borkner L, Aguiló N, Thiriard A, Coutte L, et al. IL-17dependent SIgA-mediated protection against nasal Bordetella pertussis infection by live attenuated BPZE1 vaccine. Mucosal Immunol 2018;11:1753-62. https://doi.org/10.1038/s41385-018-0073-9.

122 Goodman YE, Wort AJ, Jackson FL. Enzyme-linked immunosorbent assay for detection of pertussis immunoglobulin A in nasopharyngeal secretions as an indicator of recent infection. J Clin Microbiol 1981;13:286-92.

123 Tuomanen El, Zapiain LA, Galvan P, Hewlett EL. Characterization of antibody inhibiting adherence of Bordetella pertussis to human respiratory epithelial cells. J Clin Microbiol 1984;20:167. 
124 Redhead K, Watkins J, Barnard A, Mills KHG. Effective immunization against Bordetella pertussis respiratory infection in mice is dependent on induction of cell-mediated immunity. Infect Immun 1993;61:3190-8. https://doi.org/10.1371/journal.ppat.1003264.

125 Mc Guirk P, Mahon BP, Griffin F, Mills KHG. Compartmentalization of T cell responses following respiratory infection with Bordetella pertussis : hyporesponsiveness of lung $T$ cells is associated with modulated expression of the co-stimulatory molecule CD28. Eur J Immunol 1998;28:153-63. https://doi.org/10.1002/(sici)1521-4141(199801)28:01<153::Aidimmu153>3.0.Co;2-\#.

126 Dunne A, Ross PJ, Pospisilova E, Masin J, Meaney A, Sutton CE, et al. Inflammasome Activation by Adenylate Cyclase Toxin Directs Th17 Responses and Protection against Bordetella pertussis . J Immunol 2010;185:1711-9. https://doi.org/10.4049/jimmunol.1000105.

127 Wilk MM, Misiak A, McManus RM, Allen AC, Lynch MA, Mills KHG. Lung CD4 Tissue-Resident Memory T Cells Mediate Adaptive Immunity Induced by Previous Infection of Mice with <em>Bordetella pertussis </em>. J Immunol 2017;199:23343. https://doi.org/10.4049/jimmunol.1602051.

128 Mueller SN, Mackay LK. Tissue-resident memory T cells: local specialists in immune defence. Nat Rev Immunol 2016;16:79-89.

https://doi.org/10.1038/nri.2015.3.

129 Wilk MM, Borkner L, Misiak A, Curham L, Allen AC, Mills KHG. Immunization with whole cell but not acellular pertussis vaccines primes CD4 TRM cells that sustain protective immunity against nasal colonization with Bordetella pertussis . Emerg Microbes Infect 2019;8:169-85. https://doi.org/10.1080/22221751.2018.1564630.

130 Mattoo S, Cherry JD. Molecular pathogenesis, epidemiology, and clinical manifestations of respiratory infections due to Bordetella pertussis and other Bordetella subspecies. Clin Microbiol Rev 2005;18:326-82. https://doi.org/10.1128/CMR.18.2.326-382.2005.

131 Higgins SC, Jarnicki AG, Lavelle EC, Mills KHG. TLR4 Mediates Vaccine-Induced Protective Cellular Immunity to Bordetella pertussis : Role of IL-17-Producing T Cells. J Immunol 2006;177:7980-9. https://doi.org/10.4049/jimmunol.177.11.7980.

132 Storsaeter J, Hallander HO, Gustafsson L, Olin P. Levels of anti-pertussis antibodies related to protection after household exposure to Bordetella pertussis . Vaccine 1998;16:1907-16.

133 van der Lee S, Hendrikx LH, Sanders EAM, Berbers GAM, Buisman A-M. WholeCell or Acellular Pertussis Primary Immunizations in Infancy Determines Adolescent Cellular Immune Profiles. Front Immunol 2018;9:51. https://doi.org/10.3389/fimmu.2018.00051.

134 Ausiello CM, Urbani F, la Sala A, Lande R, Cassone A. Vaccine- and antigen- 
dependent type 1 and type 2 cytokine induction after primary vaccination of infants with whole-cell or acellular pertussis vaccines. Infect Immun 1997;65:2168-74.

135 Ryan M, Murphy G, Ryan E, Nilsson L, Shackley F, Gothefors L, et al. Distinct T-cell subtypes induced with whole cell and acellular pertussis vaccines in children. Immunology 1998;93:1-10. https://doi.org/10.1046/j.13652567.1998.00401.x.

136 da Silva Antunes R, Babor M, Carpenter C, Khalil N, Cortese M, Mentzer AJ, et al. Th1/Th17 polarization persists following whole-cell pertussis vaccination despite repeated acellular boosters. J Clin Invest 2018;128:3853-65. https://doi.org/10.1172/JCI121309.

137 Ross PJ, Sutton CE, Higgins S, Allen AC, Walsh K, Misiak A, et al. Relative contribution of Th1 and Th17 cells in adaptive immunity to Bordetella pertussis : towards the rational design of an improved acellular pertussis vaccine. PLOS Pathog 2013;9:e1003264. https://doi.org/10.1371/journal.ppat.1003264.

138 Mills KHG, Ryan M, Ryan E, Mahon BP. A murine model in which protection correlates with pertussis vaccine efficacy in children reveals complementary roles for humoral and cell- mediated immunity in protection against Bordetella pertussis . Infect Immun 1998;66:594-602. https://doi.org/10.1371/journal.ppat.1003264. 


\section{Chapter 2 - Characterizing the innate and adaptive responses of immunized mice to Bordetella pertussis infection using in vivo imaging and transcriptomic analysis}

Submitted 2019 to bioRxiv, Preprint server for Biology

Dylan T. Boehm ${ }^{\mathrm{a}}$, Melinda E. Varney ${ }^{\mathrm{a}}$, Ting Y. Wonga, Evan S. Nowak ${ }^{\mathrm{a}}$, Emel SenKilic $^{a}$, Jesse Halla, Shelby D. Bradford ${ }^{a}$, Katherine DeRoos ${ }^{a}$, Justin Bevere ${ }^{a}$, Matthew Epperly $^{\mathrm{a}}$, Jennifer A. Maynard ${ }^{\mathrm{b}}$, Erik L. Hewlett ${ }^{\mathrm{c}}$, Mariette Barbier ${ }^{\mathrm{a}}$, and F. Heath Damron $^{a^{*}}$

a Department of Microbiology, Immunology, and Cell Biology, West Virginia University, Morgantown, WV, USA

${ }^{b}$ Department of Chemical Engineering, University of Texas at Austin, Austin, TX 78712, USA

c Department of Medicine, Division of Infectious Diseases and International Health, University of Virginia, Charlottesville, Virginia, USA 


\section{Abstract}

Bordetella pertussis (B. pertussis) is the causative agent of pertussis (whooping cough). Since the 1990s, pertussis has re-emerged in the United States despite an estimated $95 \%$ vaccine coverage. Our goal was to characterize neutrophil responses and gene expression profiles of murine lungs in the context of vaccination and $B$. pertussis challenge. We utilized a bioluminescent neutrophil mouse model (NECre luc) to track neutrophil recruitment. NECre luc mice were immunized with whole cell vaccine (WCV), acellular vaccine (ACV), or a truncated adenylate cyclase toxoid (RTX) vaccine. Neutrophil recruitment was measured in live mice across time and corroborated by flow cytometry and other data. WCV immunized mice showed signs of neutrophilia in response to $B$. pertussis challenge. Mice immunized with either ACV or WCV cleared the challenge infection; however immunization with RTX alone was not protective. RNA sequencing revealed distinctive gene expression profiles for each immunization group. We observed an increase in expression of genes associated with responses to infection, and changes in expression of distinct genes in each vaccine group, providing a complex view of the immune response to $B$. pertussis infection in mice. This study suggests that combination of immunological analysis with transcriptomic profiling can facilitate discovery of pre-clinical correlates of protection for vaccine development. 


\section{Introduction}

Pertussis is a human disease primarily caused by a respiratory infection of the Gramnegative pathogen Bordetella pertussis (B. pertussis). The hallmark of pertussis is a distinctive whooping cough. What is surprising about pertussis is that the cause of the cough has never been elucidated, which highlights the fact that there are many underresearched aspects of this disease. Aerosolized B. pertussis bacterium are inhaled and adhere to airway respiratory epithelial cells through bacterial adhesins such as filamentous hemagglutinin (FHA), fimbriae and pertactin ${ }^{1}$. After colonization B. pertussis express multiple toxins including pertussis toxin (PT) and adenylate cyclase toxin (ACT). B. pertussis releases PT, which dysregulates the immune response through ADP-ribosylation of the G-protein $\alpha$-subunit of cytokine receptors present on a range of leukocytes ${ }^{2-5}$. The secretion of PT has long range effects, ACT is thought to act locally on host cells by converting ATP into supraphysiological levels of CAMP, further dysregulating the host immune response ${ }^{6}$.

In the 1940s, an effective whole cell vaccine (WCV) was developed and as a result, basic research efforts on $B$. pertussis decreased. The WCVs were highly reactogenic and caused prolonged and unusual crying after administration, hyporesponsivness, and febrile convulsions ${ }^{7-9}$. These issues led to the development of acellular vaccines (ACV), known today as DTaP/Tdap (hereafter referred to as ACV). The ACVs utilize an alum adjuvant and induce a Th2 response to several key virulence factors such as PT, FHA, fimbriae, and pertactin depending on vaccine formulations. Whereas the ACV induces a Th2 response in human and mice, WCV immunization and $B$. pertussis infection promote a Th1/Th17-type response ${ }^{10-14}$. Since the replacement of the WCV with the ACVs there has been a remarkable increase in the number of cases in the US, with the number of reported cases in 2012 equaling that of 1954. Multiple factors potentially played a role in the return of pertussis in the US. It has been documented that the Th2 response induced by ACV immunization may be inferior to the Th1/Th17 response directed by the $\mathrm{WCV}^{15}$. Additionally, ACV protection wanes significantly as early as one year after vaccination, and by 4 years after vaccination vaccine effectiveness is only $9 \%{ }^{16}$. Furthermore, in a non-human primate model it has been shown that ACV immunized baboons challenged with $B$. pertussis are capable of 
carrying the infection and transmitting aerosolized B. pertussis to naïve baboons ${ }^{17}$. Additionally, the findings of an epidemiological study suggest that asymptomatic transmission of pertussis is indeed occurring in the human population, and has a role in the increase of pertussis incidents ${ }^{18}$. These recent findings demonstrate the shortcomings of the ACV. Utilizing new technologies that were not available during the developments of the WCV or ACVs we can further investigate differences between WCV and ACV protection. Adenylate cyclase toxin (ACT) is an essential virulence factor of $B$. pertussis and a known protective antigen ${ }^{19-21}$. ACT contains two main domains: adenylate cyclase and the Repeats-in-Toxin (RTX). In the absence of the AC domain, the protein is non-toxic and referred to simply as $\mathrm{RTX}^{22}$. In a previous study, sera from mice immunized with RTX contained antibodies capable of neutralizing ACT. ACT antigens are not included in any commercial formulations of ACVs and many have proposed that its inclusion could improve protection ${ }^{6}$.

Neutrophils play a role in both the innate and adaptive arms of the immune system. Following $B$. pertussis infection of a naïve mouse, neutrophils reach peak recruitment between 5 and 7 days after challenge ${ }^{23,24}$. This occurs after the bacterial burden has begun to decrease. Subsequent studies have determined that neutrophil depletion prior to $B$. pertussis infection in naïve mice does not increase bacterial burden. However, when immunized mice are challenged with $B$. pertussis following depletion of neutrophils the $B$. pertussis bacterial burden increase, demonstrating a protective role of neutrophils in immunized mice ${ }^{25}$. As previously mentioned, $B$. pertussis toxins $\mathrm{PT}$ and ACT both affect neutrophils and their recruitment ${ }^{6,26-28}$. These toxins contribute to a significant rise in the number of circulating white blood cells and in severe cases leukocytosis is linked to fatality ${ }^{1}$. Leukocytosis was first described in pertussis patients as early as the $1890 \mathrm{~s}^{29,30}$. In the 1960's, Stephen Morse observed leukocytosis following WCV immunization ${ }^{31}$, which he hypothesized was caused by PT, then known as leukocytosis-inducing factor ${ }^{32}$. Therefore, it can be conceived that the toxin associated effects on neutrophils would be neutralized following ideal $B$. pertussis immunization, and this raises the question of where and when neutrophils are recruited in an ACV or WCV protected mouse? 
In this study, we utilized in vivo imaging systems (IVIS) to characterize the neutrophil responses to $B$. pertussis challenge in mice vaccinated with ACV or WCV. We implemented NECre luc mice, a luminescent neutrophil reporter mouse strain, previously used in a Bacillus anthracis infection model to track neutrophil recruitment following $B$. pertussis challenge ${ }^{33}$. To do so, we: 1 ) validated the NECre luc mouse as a model for tracking neutrophil recruitment in response to $B$. pertussis infection, 2) tracked the spatiotemporal localization of neutrophils in response to $B$. pertussis challenge in ACV and WCV vaccinated mice, and 3) and characterized vaccine-associated gene expression profiles of the infected lungs from each group using RNA sequencing. The NeCRE luc mouse ${ }^{33}$ was used to measure the presence and relative quantities of neutrophils in live mice during $B$. pertussis challenge. We then corroborated our findings with flow cytometry analysis to quantify neutrophils in the blood and airway of naïve and immunized mice. WCV immunization resulted in robust neutrophil recruitment and clearance of $B$. pertussis, but also resulted in morbidity of the NeCre luc mice. RNAseq analysis was performed on the lungs of naïve or immunized mice at both early and late time-points after challenge with $B$. pertussis. We observed specific gene signatures for each vaccine, and we also identified gene expression profiles that had not been associated with $B$. pertussis or immunization. Neutrophil specific gene expression corroborated our cellular analysis of the respiratory tract demonstrating increased expression of neutrophil specific genes in WCV immunized mice. Furthermore, we determined the T-helper cell gene expression profiles of lung transcriptomes and corroborated the activation of Th1 specific gene expression to increased levels of Th1associated cytokine production in the lungs of WCV immunized mice. Utilizing the depth of data generated through RNAseq, we could classify specific B cell clonotypes present in the lung of each immunization group. This technique allowed for analysis of the diversity and frequency of the immunoglobulin repertoire of immunized groups. We hypothesize that these approaches can continue to be applied to other pathogen to host interactions to characterize the underpinnings to disease progression and immunological responses. 


\section{Results}

ACV and WCV immunization of NECre luc mice results in clearance of $B$. pertussis, however WCV immunized mice experience increased morbidity and mortality.

To characterize the effects of vaccination on neutrophil recruitment, NECre luc mice were immunized with the vaccines as indicated in Supplementary Table S1, and then boosted with the same vaccine 21 days later by intraperitoneal injection. We then compared the neutrophil recruitment in naïve, ACV, WCV, and RTX-only vaccinated NECre luc mice during $B$. pertussis challenge. At day 35 post initial immunization, NECre luc mic were challenged with virulent $B$. pertussis strain UT25 (Fig. 1) by intranasal instillation. A challenge dose of $2 \times 10^{7} \mathrm{CFUs} B$. pertussis does not typically cause morbidity in immunocompetent mice such as CD-1 or C57B6/J (data not shown). We originally planned to euthanize 4 mice per group on each experimental day $(1,2,4$, and 9). However, 2 of 13 naïve challenged NECre luc mice were euthanized at day 2 due to morbidity (Fig. 2). At day 6, we observed additional unexpectedly morbidity in the WCV group, which required euthanasia of all remaining WCV immunized NECre luc mice (Fig. 2). Conversely, no morbidity was observed in the ACV and RTX immunized mice 9 days post $B$. pertussis challenge (Fig. 2). We were surprised to observe morbidity in the WCV immunized mice. Upon determining viable bacterial burden from lung, trachea, and nasal lavage, we observed clearance of $B$. pertussis to our limits of detection in both ACV and WCV immunized mice compared to naïve infection (Fig. 3abc). Taken together, these data suggested that morbidity of the WCV immunized mice was not fully attributed to bacterial burden. Additionally, we determined that vaccination with RTX resulted in 100 percent survival out to 9 days post-challenge $(\mathrm{pc})$, while bacterial burden were similar to naïve infected mice, suggesting that RTX immunization did not improve bacterial clearance (Fig. 3abc).

\section{Analysis of serological responses of immunized mice.}

As expected, the ACV immunized mice were protected against challenge as they rapidly cleared the $B$. pertussis challenge dose (Fig. $3 a b c$ ). WCV immunized mice cleared $B$. pertussis, but this response was delayed compared to the ACV mice. To determine the 
efficacy of immunization on antibody production, we performed serology analysis. PT is a common antigen of both the ACV and WCV and we observed a significantly higher concentration of anti-PT in the ACV mice compared to the WCV (Fig. 3d). Immunization of NECre mice with RTX with alum adjuvant resulted in high anti-RTX antibody titers (Fig. 3e) despite the fact that we did not see increased clearance due to RTX immunization. In another study, we immunized CD1 mice with RTX and alum adjuvant and we also saw no clearance of $B$. pertussis compared to non-vaccinated mice (data not shown). Our data suggest that, while immunization with RTX induces antibody production, it is not sufficient to protect mice from $B$. pertussis infection as a single, antigen vaccine.

\section{Spatiotemporal IVIS imaging of neutrophils in naïve and vaccinated NECre luc mice.}

Neutrophil accumulation in NECre luc mice were visualized on days 1, 2, 4, 6 and 9 post-challenge. Mice were IP injected with the high sensitivity luciferin CycLuc1 ${ }^{33}$ before imaging. A Lumina II IVIS (Xenogen) was used to capture luciferase-driven neutrophil luminescence at indicated time-points (Fig. 4 and Supplementary Fig. 1). Luminescent signal of the whole body (Fig. 4d) or nasal cavity (Fig. 4e) of vaccinated mice was then compared to signal from naïve, non-infected mice and calculated as fold change. At day 1 post-challenge, we detected significantly higher luminescence in naïve challenged mice compared to naïve non-challenged mice, suggesting that there is a higher neutrophil accumulation in challenged mice. Furthermore, whole body luminescence in WCV immunized and challenged mice was significantly higher than the whole body luminescence of naïve, ACV and RTX B. pertussis challenged mice at day 1 (Fig. 4d). In general, luminescence signals of all groups decreased across days 2 and 4. However, in the WCV group at day 6 , we detected an increase in signal in the nasal cavity (71-fold) and the whole body (47-fold) compared to naïve not challenged mice (Fig. 4de). Due to variability between animals, this increase was not significant compared to other groups. At this point WCV immunized mice were morbid and required euthanasia. Taken together this data suggests that WCV immunization prior to challenge resulted in increased neutrophil accumulation compared to other vaccination 
groups. We next aimed to corroborate our IVIS luminescence data with flow cytometry analysis of respiratory tissues.

\section{Validation of neutrophil infiltration in respiratory tissue by flow cytometry}

Luminescent signal of luciferase-expressing cells correlates with the localization of neutrophils in NECre luc mice ${ }^{33}$. To confirm this, we measured the relative number of neutrophils by performing flow cytometry analysis on respiratory tissue following IVIS imaging. Single cell suspensions from lung tissue or nasal lavage were labelled with antibodies recognizing cell surface markers. We defined neutrophils as CD11b+GR-1+ live cells (Supplementary Fig. 2). Similar to the IVIS data, all B. pertussis challenged groups had significantly higher neutrophil percentages in nasal lavage compared to naïve non-challenged mice (Fig. 4f). At day 1 post $B$. pertussis challenge, WCV immunized mice had a significantly higher percentage of neutrophils than ACV immunized mice in nasal lavage. The percentages of neutrophils decreased from day 1 to day 2 in all vaccinated groups, as well as the naïve group. At day 4, WCV immunized mice exhibited higher levels of neutrophils than those observed at day 2 (Fig. 4g). Correlating with the nasal lavage, WCV mice exhibited significantly higher percentages of neutrophils in the lungs than ACV mice. Lung neutrophils at day 4 were not statistically significant between any of the groups. However, by day 6 there was a 53\% increase in lung neutrophils compared to day 4 in the WCV group, which directly correlates with the increased luminescence signals measured by IVIS imaging (Fig. 4g).

WCV immunized NECre luc mice induce a more robust Th1 immune response compared to ACV immunized mice.

It has been previously documented that upon B. pertussis challenge, WCV immunized mice elicit a Th1/Th17 immune response, similar to a naïve infection while ACV immunized mice generate primarily a Th2 response ${ }^{10-12,34}$. We hypothesized that similar responses are triggered by WCV and ACV in the NECre luc model. To test this hypothesis, we determined the cytokine profiles in lungs of immunized and challenged mice using electrochemiluminescent sandwich immunoassays. The Th1 response was determined by increased levels of IFN- $\gamma$, IL-12p70, IL-1 $\beta$ and TNF- $\alpha$ (Fig. 5ab), while 
the Th17 response was qualified by increased IL-17A and IL-6 (Fig. 5cde). Th2 responses were associated to increased levels in IL-4 and IL-5.

As expected, we observed an increased Th1 response in the WCV vaccinated NECre luc mice compared to the ACV group. We also observed an increase in the Th1 response in naive challenged mice compared to non-challenged mice or the ACV group. Interestingly, the highest levels of IL-17 were observed in WCV NECre luc mice (Fig. 5c). The levels of Th2-associated cytokines, IL-4 and IL5 were similar between the WCV and ACV groups. (Supplementary Fig. 3). The lung cytokine profile from NECre luc mice immunized with RTX resembled a profile of naïve $B$. pertussis challenged mice with the exception of IL-6, which was lower in the RTX group. Taken together these data from the NECre luc mouse model corroborates previous findings by displaying a strong Th1/Th17 response in response to $B$. pertussis in WCV vaccinated mice.

Characterizing the gene expression profiles of the lungs of immunized and naïve mice after $B$. pertussis challenge

Current and past $B$. pertussis immune response studies have focused on a limited set of known immunological responses to $B$. pertussis challenge such as: bacterial burden, antibody response, recruitment of phagocytes, cytokine profiles, and others ${ }^{35,36}$. Whooping cough is a toxin-mediated disease, due mainly to the activity of pertussis and adenylate cyclase toxins. These toxins are released and affect a wide array of host cells $^{4,25}$. It is therefore conceivable that $B$. pertussis infection would have a broader effect on the respiratory tissue beyond the induction of predictable immune response factors. To address this, we employed RNA sequencing (RNAseq) to observe the effect of $B$. pertussis challenge on the lungs of immunized and naïve NECre luc mice at a transcriptomic level. This allowed us to characterize the transcriptome response to infection and vaccine-induced responses. We hypothesized that regardless of the vaccine administered, we would identify a set of genes differentially regulated in all challenged mice. Additionally, we sought to discern the unique gene expression profiles in response to challenge in each immunized group.

To perform RNAseq analysis, lungs from NECre luc mice were harvested at an early time-point (day 1 ) following $B$. pertussis challenge and at a late time-point (day 6 or 9). 
RNA from the lungs of the mice was isolated. Libraries were prepared and sequenced on the Illumina HiSeq 1500 platform. To characterize transcriptional responses to $B$. pertussis challenge in naïve or vaccine mice (ACV, WCV, RTX), we compared the challenged samples to control mice that were not immunized nor challenged. Based on this, we expected to determine both the immunological (innate and adaptive) and the non-immunological responses to $B$. pertussis challenge. We determined the differentially expressed genes (DEGs) for both early (day 1 ) and late (day 6 or 9 depending on group) time-points. The total numbers of differentially regulated genes are shown in Fig. 6ac. Overall, there were more differentially genes at the later time-point than early after challenge (Fig. 6ac). At day 1 post-challenge, the WCV group had the most genes activated (Fig. 6a, Supplementary Fig. 5). Of the genes that were upregulated at day 1 in the WCV, 29.7\% were not found upregulated in the other groups (464 of 1,560). At the late time-points, there were more common upregulated genes and only $14.1 \%$ of the genes were unique to the WCV. We found that $12.6 \%$ of unique upregulated genes (196) were common to all groups either challenged with $B$. pertussis following immunization or naïve challenge, suggesting that these genes were common to infection (Fig. 6b). At the later time-point, 19.8\% of unique upregulated genes (867 of $6,595)$ were shared by the WCV, ACV, RTX groups, while this number was much lower at the early time point (8.5\% of DEGs, 133 of 1,560) (Fig. 6bd). Cumulatively, this data suggests that: 1) WCV vaccination induces a more diverse early response to $B$. pertussis challenge due to the higher number of unique genes compared to other groups, and 2) at the later time-point all vaccinated groups have a more similar lung transcriptome.

Ingenuity Pathway Analysis (IPA) was performed to determine enrichment analyses of gene expression signatures in the context of each immunization. Using IPA Knowledgebased enrichment analysis, statistically significant genes were grouped into disease and function categories. A system of genes was considered enriched based on Fisher's exact test on the ratio of represented genes. At the early time-point we, observed that the largest changes were associated with genes involved in immune cell trafficking of the WCV group (Fig 7a). Genes with the highest fold changes within this system were associated with activation and migration of leukocytes (Fig. 7b). Additionally, we 
observed significant enrichment of genes associated with the humoral and cellmediated immune responses (Fig. 7b). Pertussis toxin affects multiple aspects of the cardiovascular system such as blood pressure, leukocyte migration, etc ${ }^{7,37,38}$. In both naïve and immunized mice, we observed gene expression signatures related to cardiovascular diseases (Fig. 7ab). ACV immunization results in high amounts of antiPT antibodies (Fig. 4d), and we observed fewer genes significantly altered in the ACV immunized mice, suggesting positive effects of anti-PT antibodies on neutralization of toxin activity. At the late time-point, immune cell trafficking gene expression seemed to decrease in naïve, ACV, and RTX groups; however, the expression of these genes was still high in the WCV group. As expected, immune-related gene signatures dominated the responses to $B$. pertussis infection. However, thousands of genes significantly dysregulated due to infection at both time-points were not related to immune response or were not sufficiently characterized to be assigned a function. Analysis of the lung transcriptomes corroborated the findings of flow cytometry and cytokine profile analyses, further supporting that there is an increase in leukocyte activity in the respiratory tissue of WCV immunized mice compared to ACV or RTX immunized mice following $B$. pertussis challenge.

\section{Analysis of neutrophil-specific gene signatures}

Since we observed that neutrophils accumulate in the lung of WCV immunized NECre luc mice, we chose to delve further into the changes in expression of genes associated with neutrophil activity. To compare the neutrophil response between the groups, we selected genes with GO terms associated with neutrophil migration and activation and plotted the relative fold changes for this subset of genes for each immunization group (Fig. 8). The expression levels of these genes from either the naïve challenged, or immunized and challenged groups was compared to naïve, not infected control mice (Fig. 8).

$\mathrm{Cxcl} 2$ is a chemokine produced by monocytes and neutrophils at sites of infection ${ }^{39}$. Previously it has been shown that convalescent mice previously challenged with $B$. pertussis express lower amounts of $c x c / 2$ when re-challenged with $B$. pertussis than naïve mice ${ }^{40}$. This suggests that the adaptive responses in these mice negate the need 
for the innate production of $\mathrm{Cxcl} 2$. Supporting this hypothesis, we observed the highest expression levels of $\mathrm{Cxcl} 2$ in naïve and RTX immunized mice, which had similar bacterial burdens (Fig. 7bd and Fig. 8be). The induction of $C x c / 2$ expression was lower in ACV vaccinated mice than naïve mice (Fig. 8ac). The expression of $\mathrm{Cxcl} 2$ also increased in the WCV mice, but remained high at the late time-point (Fig. 8b). Our serological analysis indicated that WCV immunization only induced minimal anti-PT production, whereas ACV induced high amounts of anti-PT (Fig. 3d). PT has been shown to induce production of Cxcl2 in TY10 cerebral endothelial cells which further suggests a causal relationship between non-neutralized PT and Cxcl2 production ${ }^{41}$.

All of the groups showed increases in a number of other $\mathrm{C}-\mathrm{X}-\mathrm{C}$ and $\mathrm{C}-\mathrm{C}$ family chemokines (cxc/3, cxcl1, cc/3, ccl4, cc/6, and cc/17). Cxcl1 gene expression was increased at day 1 in all groups compared to the non-infected control (Fig. 8). We also observed an increase in IL-23 expression in the WCV group (Fig. 7b), which might be associated with the IL-17 cytokine response measured in the WCV lung tissue. Tumor necrosis factor (tnf) gene expression was increased in WCV, RTX, and ACV mice compared to non-infected mice. However, Tnf expression levels decreased in ACV mice and were lower than those observed in uninfected mice at day 9 (Supplementary Fig. 4). Ross et al. demonstrated that IL-17A promotes production of CXCL1 during $B$. pertussis challenge ${ }^{10}$. In our cytokine analysis, only the WCV mice had sufficient IL-17 to be detected in the lung homogenates (Fig. 5c). Taken together these data suggest that although we did not observe high IL-17A gene expression in the transcriptome or in the IL-17 cytokine analysis, there could still be sufficient IL-17A in the Naïve, ACV, and RTX challenged mice that could account for the increased $\mathrm{Cxc} / 1$ gene expression.

We observed that S100a9 and S100a8 were highly upregulated in WCV and RTX groups (Fig. 7 and Fig. 8b). S100a9 is a major calcium-binding protein of neutrophils, which forms a heterodimer with S100a8, and promotes polymerization of microtubules. Interestingly, S100a8/a9 is produced mainly by neutrophils and high plasma levels of S100a8/a9 correlate with higher blood neutrophil blood levels in heathy human adults ${ }^{42}$. S100a8/a9 promotes transendothial phagocyte migration by promoting tubulin polymerization ${ }^{43}$. Additionally, monocytes isolated from human patients with cardiac 
injury have been found to be particularly responsive to S100a8/a9 and secrete increased amounts of TNF- $\alpha$ and IL-6 ${ }^{44}$. S100a8/a9 can induce neutrophil chemotaxis and adherence, but PT can block this function of S100a8/a945. These data suggest that PT induction of leukocytosis may be related to the role of S100a8/a9 in leukocyte recruitment. S100a8/a9 expression was lowest in the ACV mice which had the highest amount of serum anti-PT.

Overall the WCV mice had higher expression of more neutrophil genes than any of the other groups (Fig. 7). Expression of these neutrophil signatures did not return to uninfected levels. These observations corroborates IVIS (Fig. 4de) and flow cytometry analyses (Fig. 4fg). Neutrophil gene expression in ACV mice was dynamic and returned to levels lower than uninfected mice by day 9. ACV mice did not have detectable $B$. pertussis in their lungs at day 9 , indicating that the initial challenge does had been cleared (Fig. 3). ACV and WCV signatures were the most dissimilar, while the RTX and naïve mice transcriptome profiles were the most similar (Fig. 7). This correlates with the fact that RTX mice still have detectable $B$. pertussis in their nares, trachea and lungs at day 9 at levels similar to those observed in non-vaccinated mice. These data confirm our neutrophil observations but also enhance our analysis of the neutrophil dynamic in the context of vaccination status and $B$. pertussis challenge.

\section{B cell gene expression signatures in relation to immunization status.}

It has been established that $B$ cells are required for the clearance of $B$. pertussis in mice and that ACV immunization protects through neutralizing antibodies ${ }^{46,47}$. To further investigate antibody production between WCV and ACV immunized mice, we aimed to determine the diversity of $B$ cell clone expansion between vaccinated and nonvaccinated challenged mice. Using MiXCR software, the same illumina sequenced reads mentioned above were analyzed specifically for immunoglobulin profiling ${ }^{48}$. Individual B cell clones were identified by B cell receptor sequence and clonal expansion was accessed by immunoglobulin diversity and frequency between the vaccinated and challenged, naïve challenged, and non-challenged control groups. At the early time-point day 1 post-challenge, immunoglobulin diversity and frequency of $B$ cells depicted a close relation between the ACV and RTX vaccination groups, as also 
did WCV and naïve challenged mice (Supplementary Fig. 6a). This response shifted by the late time-point (days 6 or 9), when we observed that WCV and ACV groups were more similar. Using this approach, we were able to determine the individual variable and joining segments abundance, and the frequency of which a particular was found. Not surprisingly, we observed the highest degree of diversity at the late time-point. WCV and ACV were the most similar due to a higher number and abundance of clonotypes (Supplementary Fig. 6b). We focused our analysis on the diversity of the third complementarity-determining region (CDR3) of the heavy Ig chain, because this region has been shown to be necessary for antigen specificity ${ }^{49}$. The highest abundant CDR3 region sequence, NQHLFW, was present only in the WCV immunized mice, while the other 10 highest CDR3 regions were shared across vaccine groups at the early and late time-points (Supplementary Table 6 and Supplementary Table 7). Together this data suggests that clones generated from acellular based vaccines (ACT and RTX) are more closely related because they are generated to a limited number of antigens. However, by the late time-point, proliferation of specific B cell clones generated from WCV has created the same diversity seen in the ACV clones. The B cell clonotype analysis suggests that we can aim to further understand these populations in the future, but we will need to increase our sequencing depth by directly isolating the cell populations from the immunized mice instead of analyzing the full lung tissue. It may also be interesting to probe the $T$ cell receptor clonotypes using the same method.

\section{Discussion}

In this study, we compared the innate and adaptive immune responses of immunized NECre luc mice vaccinated with ACV or WCV vaccines, to mice immunized with a truncated ACT toxin absorbed to alum (RTX), or non-vaccinated naïve mice following challenge with $B$. pertussis. ACV and WCV immunized mice cleared $B$. pertussis challenge but distinctive immune responses were observed. We also determined that the RTX antigen alone was not protective as a single antigen vaccine with alum adjuvant in NeCre luc mice. We utilized IVIS imaging to track the recruitment of neutrophils to the respiratory tract of challenged mice from days 1 to 9 post-challenge. The data obtained illustrated a dramatic increase in the amount of neutrophils recruited 
to the lungs and nasal cavity of WCV immunized mice compared to ACV immunized mice when followed by $B$. pertussis challenge. These data were also supported by flow cytometry and cytokine analysis. Furthermore, by performing RNA sequencing on the lung transcriptome during infection in vaccinated or naïve mice, we described unique gene expression profiles depending on the vaccination status of the mice. We also used RNA sequencing to begin to describe the immunoglobulin diversity induced by each vaccine.

Upon initial B. pertussis challenge, we observed activation of neutrophil associated genes in all groups, however it was only in the WCV immunized mice that we saw increased neutrophil genes expression still elevated at the late time-point. Neutrophilia and elevated neutrophil related genes expression at the late time-point, when bacterial numbers in the airways are greatly reduced, indicates either over activation of neutrophil "chemokine storm," or possibly a lack of neutralization of bacterial toxins. Multiple studies suggest that PT is responsible for neutrophilia and leukocytosis following $B$. pertussis infection ${ }^{13,32,50,51}$. Immunization with the ACV induced 1,146-fold more anti-PT antibody production than WCV immunization at day 2 (Fig. $3 d$ ). It is possible that the low levels of anti-PT production in WCV mice resulted in insufficient neutralization of PT, resulting in exacerbated neutrophilia. Surprisingly, the highest neutrophil accumulation was determined at day 6 post-challenge when bacterial levels were similar to those of ACV protected mice (Fig. 3). These findings suggest that while WCV immunization is protective at clearing infection in NECre luc mice, it also induced severe neutrophilia. Interestingly, the neutrophil response in WCV mice decreased at days 2 and 4 but highly increased at day 6 (Fig. 4). At day 2, we observed a significant increase in IL-17 in the lungs of WCV mice (Fig. 5c) that could result from the increased IL-17 expression in these mice. Stephen Morse observed dose-dependent leukocytosis in mice following vaccination with killed $B$. pertussis ${ }^{31}$. In this study, NECre luc mice were immunized with $1 / 5^{\text {th }}$ the human dose of WCV. We now realize that this dose is likely well above the proportional weight of a mouse compared to a human. It is possible that this high vaccine dose induced the hyper leukocytosis, similar to the dosedependent increase that Morse observed. It is known that hyperleukocytosis is associated with death in infant cases, and that PT is responsible for inducing 
leukocytosis ${ }^{1,7,52}$. Furthermore, mice and baboons infected with $B$. pertussis and then treated with anti-PT antibodies had lower levels of leukocytosis compared to noninfected controls ${ }^{50}$. An epidemiological study found that in unvaccinated individuals with pertussis, $72 \%$ of patients experienced leukocytosis ${ }^{53}$. In this study, we observed that WCV immunized NECre luc mice experienced neutrophilia and morbidity, which highlights the potential issues of using WCVs. It would be interesting to determine the relationship of neutrophilia and WCV immunization in epidemiological past studies but we have not been successful in identifying a study that specifically looked at neutrophilia because studies most note general leukocytosis.

In NECre luc mice, the ACV was clearly more protective and less detrimental to the mice than the WCV. Truncated and full-length ACT purified from B. pertussis culture has been shown to be a protective antigen ${ }^{21}$. Wang et al. described the RTX region of ACT as highly immunogenic and easily purified as a recombinant protein ${ }^{22}$. We have observed that immunization of CD1 mice with RTX and alum adjuvant results in high anti-RTX titers (data not shown). Here, we immunized NECreluc mice with RTX and alum, but no protection was observed (Fig. 3abc). The previous studies were performed with strain 18323 and the antigen was directly isolated from $B$. pertussis ${ }^{21,54}$. It is now known that strain 18323, is an outlier compared to most other global $B$. pertussis strains and Guiso et $\mathrm{a}^{21}$. noted that strain 18323 produces less PT than the Tohama I type strain. From our work, we know that UT25 produces more PT and ACT than Tohama I (data not shown). It is possible that due to the increased PT levels produced by UT25, vaccination with RTX was not sufficient to block colonization and proliferation in vivo. Although RTX immunization did not result in clearance of $B$. pertussis from NECre luc mice, we did observe a reduction in the pro-inflammatory cytokine IL-6 in the RTX group compared to not vaccinated and WCV vaccinated and challenged mice (Fig. 5e). PT and ACT have both been shown to induce the production of IL-6 in human cell lines ${ }^{55,56}$. The anti-ACT antibodies generated by RTX vaccination may play a role in reducing the levels of IL-6 due to reducing the activity of ACT. We hypothesize that it is necessary to neutralize both PT and ACT to provide optimal protection and in future studies, we will test RTX as an antigen in a multivalent ACV containing PT antigen. 
Using standard immunological analyses, we observed typical Th2 and Th1/17 responses in ACV and WCV immunized mice respectively. $B$. pertussis utilizes PT and ACT to facilitate survival in the host and most studies about the PT/ACT specific effects have been perform on cell cultures in vitro. Microarray analysis has been used to profile the lung transcriptome of mice challenged with $B$. pertussis ${ }^{40,57}$. Here, we sought to use RNAseq to investigate the overall gene expression profiles of the murine lung in response to $B$. pertussis challenge. Analysis of the lung transcriptome 1 day after $B$. pertussis challenged exhibited a distinct profile in mice vaccinated with WCV compared to ACV, RTX, or naïve infected mice. This response was consistent with our WCV cytokine profile demonstrating a strong pro-inflammatory response. Similarly, transcriptome data from others during early $B$. pertussis infection noted an increase in chemokines such as $\mathrm{Cxcl} 2, \mathrm{Cxcl} 10, \mathrm{Cxcl} 3, \mathrm{Ccl} 3, \mathrm{Cxcl} 1$, and $\mathrm{Ccl} 4{ }^{57,58}$. In Raeven et al, convalescent mice previously infected with $B$. pertussis exhibited higher expression of these chemokines compared to naïve mice, similar to our whole cell transcriptome where these chemokines are consistently higher than the naïve mice (Fig. 7 and Fig. 8). We observed the highest expression of these chemokines in the naïve and RTX mice groups, where these groups had a similar gene profile of genes belonging to the immune cell trafficking and cardiovascular disease annotations (Fig. 7). These findings, along with higher bacterial burden (Fig. 3) suggest that RTX-alum immunization does not provide sufficient protection.

Our overall transcriptomic profiling revealed thousands of significant gene expression changes (Fig. 6). After analyzing the neutrophil (Fig. 8), and innate gene changes (Supplementary Fig. 4), we then sought to specifically characterize B cell clone diversity. The RNAseq reads were re-processed with the MiXCR algorithm and we analyzed the abundance and diversity of VDJ clonotypes (Supplementary Fig 5). Besides the $T$ cell response differences of the ACV and WCV (Th2 V. Th1/Th17) another significant difference between these two vaccines is the number of antigens. ACVs have 3-5 antigens (PT, FHA, PRN, FIM2/3) but the WCV hypothetically has $\sim 3,000$ antigens. In light of this, it would be logical to hypothesize that the WCV would induce a vast antibody repertoire as measured by many VDJ clonotypes. However, we observed a greater diversity and abundance in the ACV group compared to the WCV at 
both early and late time-points (Supplementary Fig. S6). It is important to point out that this analysis was performed on total lung RNA. If we were to isolate B cells and sequence deeper we would expect to more thoroughly characterize the repertoire. These data are interesting but we do not know which clone types result in functional antibodies that protect against $B$. pertussis. Further analysis is required to bridge the gap between clonotypes and functional/protective antibodies.

Using NeCRE luc mice, we performed tracking neutrophil recruitment during a $B$. pertussis respiratory infection. These data demonstrate how analysis of cellular responses through in vivo imaging can be used to provide a quantitative parameter throughout a study. This model can be applied to other bacterial infection models or cancer tumor progression models where following the same mouse throughout a study would be beneficial. In this study, we also added next generation sequencing technology to expand upon the immunological findings. RNAseq analysis can be further employed to understand key cell populations and how they are impacted by both immunization and $B$. pertussis challenge. Our current goal is to continue to refine the murine challenge models with new technological approaches in order to facilitate formulation of new pertussis vaccines. The findings of this study suggest that by integrating RNAseq analysis with classic immunological techniques, it is possible to illuminate novel intricacies of vaccine induced immunity to $B$. pertussis.

\section{Methods}

\section{Bacteria and culture conditions}

B. pertussis strain UT25 (UT25Sm1) were cultured on Bordet-Gengou (BG) agar (1906) supplemented with 15\% defibrinated sheep blood (Hemostat Laboratories) for $48 \mathrm{~h}$ at $36^{\circ} \mathrm{C}^{59}$. B. pertussis was then transferred from BG plates to three flasks of $12 \mathrm{ml}$ of modified Stainer-Scholte liquid medium (SSM) ${ }^{60}$. SSM cultures were not supplemented with cyclodextrin (Heptakis(2,6-di-O-methyl)- $\beta$-cyclodextrin). SSM cultures were grown for $\sim 22 \mathrm{~h}$ at $36^{\circ} \mathrm{C}$ with shaking at $180 \mathrm{rpm}$ until the OD600 reached 0.5 on a $1 \mathrm{~cm}$ path width spectrophotometer (Beckman Coulter DU 530). The cultures were then diluted to $1 \times 10 \% \mathrm{ml}$ with SSM. 


\section{Mouse Strains}

All mouse strains used were bred in a specific pathogen-free experimental conditions within the Office of Laboratory Animal Resources vivarium at West Virginia University. Mice were aged 8-12 weeks, male and female sex mice were equally assigned to all vaccination groups. 129-Elane ${ }^{\mathrm{tm} 1(\mathrm{cre}) \mathrm{Roes}} / \mathrm{H}$ mice (Medical Research Council, London, UK) were crossed with FVB.129S6(B6)-Gt(ROSA)26Sortm1(luc)kael/J mice (Jackson labs; 0051225) resulting in NECre luc progeny. NECre luc mice were injected with CycLuc 1 lucferin (EMD Millipore, Darmstadt, Germany) and confirmed to be luminescent using Xenogen Lumina $\|^{61}$. Upon intraperitoneal injection of a luciferase substrate (luciferin), neutrophils emit luminescence that is detectable using a high sensitivity live animal imaging system (Xenogen IVIS Lumina II).

\section{Vaccines used in study and administration}

All vaccines were formulated into $200 \mu \mathrm{l}$ doses with the antigen content described below. $100 \mu$ of INFANRIX (GSK) human vaccine (DTaP) which is 1/5 human dose of the vaccine, was mixed in $100 \mu \mathrm{l}$ of PBS. The NIBSC WHO standard Bordetella pertussis whole-cell vaccine (NIBSC code 94/532) was received lyphophilzed and reconstituted in $1 \mathrm{ml}$ of PBS. At this concentration one human dose is $100 \mu$ l therefore, $20 \mu \mathrm{l}$ was mixed with $180 \mu \mathrm{l}$ of PBS, which is $1 / 5$ of the human dose (66 $\mu \mathrm{g}$ of total protein). A truncated ACT protein (RTX) was purified as previously described ${ }^{22} 5.6 \mathrm{ug}$ of RTX was combined with $100 \mu \mathrm{l}$ alum adjuvant (Alhydrogel $₫$, InvivoGen) corresponding to $1 \mathrm{mg}$ of aluminum hydroxide. All immunizations occurred by intraperitoneal injection. Unvaccinated mice received $200 \mu \mathrm{l}$ of sterile PBS.

\section{Vaccination and Challenge with Bordetella pertussis}

NECre luc mice were bred to ages ranging from (8-12 weeks). Mice were vaccinated at day 0 , and then boosted 21 days later. Thirty-five days post initial vaccination, $B$. pertussis UT25 was grown as described above, and provided as a challenge dose at $2 \mathrm{x}$ $10^{7} \mathrm{CFU}$ in $20 \mu \mathrm{l}$. Mice were anesthetized by intraperitoneal injection of $200 \mu \mathrm{l}$ of ketamine $(6.7 \mathrm{mg} / \mathrm{ml})$ and xylazine $(1.3 \mathrm{mg} / \mathrm{ml})$ in $0.9 \%$ saline. Two $10 \mu \mathrm{l}$ doses of bacteria were administered through nasal inhalation into each nostril of the mouse. Mice 
from each of the groups were challenged WCV (8), ACV (8), RTX (6), and naïve control (PBS injection) (5).

\section{Collection of murine samples and determination of bacterial burden}

On days 1,2,4,6, and $9 \mathrm{pc}$, mice were euthanized by intraperitoneal injection of pentobarbital and dissected in a biosafety cabinet under BSL-2 conditions. Blood was collected by cardiac puncture, serum was separated by centrifugation through a BD Microtainer SST blood collector (BD), or blood for complete blood cell counts were collected in BD Microtainer Tubes with $\mathrm{K}_{2}$ EDTA (BD). Trachea and lungs were removed, placed in $1 \mathrm{ml}$ PBS, and then homogenized. Trachea tissue was homogenized by a Brinkman Homogenizer (Polytron), while lung tissue was dissociated using a Dounce homogenizer (Kimble Chase). To determine viable B. pertussis in the nares, $1 \mathrm{ml}$ of PBS was flushed through the nares and collected. In order to determine bacterial burden $100 \mu \mathrm{l}$ of homogenate or nasal lavage was serial diluted in sterile PBS. Four $10 \mu \mathrm{l}$ aliquots of each serial dilutions were plated on BG containing streptomycin $(100 \mu \mathrm{g} / \mathrm{ml})$ to ensure only UT25 $\mathrm{B}$. pertussis grew on the plates. After $72 \mathrm{~h}$ at $36^{\circ} \mathrm{C}$ colony forming units (CFUs) were counted and the bacterial burden per tissue was calculated. Due to the serial dilutions plated our limit of detection was $10^{3} \mathrm{CFUs}$ per $\mathrm{ml}$ or organ. CFUs from experimental groups at each time-point were compared to the naive (PBS injected) and challenged group by a two-tailed unpaired t test using the software package Prism 7 (GraphPad, La Jolla, CA). All experiments were performed in accordance with the National Institutes of Health Guide for the Care and Use of Laboratory Animals. All murine infection experiments were performed per protocols approved by the West Virginia University Institutional Animal Care and Use Committee (protocol number Damron 14-1211).

\section{IVIS imaging}

NECre luc mice received $83 \mathrm{mg} / \mathrm{kg}$ of CycLuc1 luciferin by IP injection $(100 \mu \mathrm{l})$. Five minutes after injection mice were anesthetized with $3 \%$ isoflurane, mixed with oxygen from the XGI-8 gas anesthesia system supplied with a Xenogen IVIS Lumina II. Luminescent signals were acquired during a five-minute exposure. Acquisition was performed using Living Image 2.5 software (Xenogen). Images were acquired with a 
binning of 4. Following imaging, mice were either euthanized for dissection and extraction of tissue samples or returned to the vivarium. Luminescence was determined by quantification of photons emitted per second generated in each region of interest (ROI): whole mouse image or nasal cavity. The background of each image was subtracted from the ROI, background photons were defined by the average of two ROIs away from the mouse, for the same image. Data was expressed as relative fold change between target ROIs from experimental groups to an average of ROls from control mice that were not vaccinated or challenged. Group comparisons were analyzed by one-way analysis of variance (ANOVA) followed by a Tukey's multiple-comparison test using Prism 7.

\section{Preparation of tissue and flow cytometry analysis}

Blood, lung, and cells isolated from nasal lavage were analyzed by flow cytometry. A $100 \mu \mathrm{l}$ sample was removed from lung homogenate of all samples and filtered through a $70 \mu \mathrm{m}$ cell strainer, then centrifuged at $1000 \times g$ for 5 mins to pellet cells. Supernatant was removed, and RBC lysis buffer (BD Pharm lysis) added incubated at $37^{\circ} \mathrm{C}$ for $2 \mathrm{~min}$, then pelleted using the same centrifugation conditions. Cells were resuspended in 500 $\mu \mathrm{l}$ of PBS $+1 \%$ FBS, $100 \mu \mathrm{l}$ was aliquoted for antibody staining. After cardiac puncture blood was placed in EDTA containing tube (BD Bioscience), RBC were lysed using Pharmlyse (BD Biosciences) with a 15 min room temperature incubation and then prepared in a similar manner to other tissues. All cell suspension samples were incubated on ice in PBS and 1\% FBS for blocking. Antibodies against specific cell surface markers: PE-conjugated GR-1 (BD, 553128) Alexa Fluor 700-conjugated CD11b (Biolegend, 101222) were added to cell suspensions and incubated in the dark for $1 \mathrm{~h}$ at $4^{\circ} \mathrm{C}$. Lung, blood, and nasal wash suspensions were pelleted, and resuspended in PBS prior to analysis. Samples were read using LSR Fortessa (BD), and analyzed using FlowJo v10 (FlowJo, LLC). PMNs were classified as CD11 b+Gr-1 ${ }^{+}$ single, live cells.

\section{Cytokine Quantification}

Lung homogenates were pelleted by centrifugation and then supernatant was collected and stored at $-80^{\circ} \mathrm{C}$ until analysis. Concentration of cytokines in the lungs of vaccinated 
and challenged mice were determined by quantitative sandwich immunoassays, Meso Scale Discovery (Rockville, MD) V-PLEX Proinflammatory Panel (K15048G-1) and Mouse IL-17 Ultra-Sensitive kits (K152ATC-1), following manufacturer's instructions. Data was analyzed by one-way ANOVA, with a Tukey's multiple-comparison test for each time-point.

\section{Serology}

Vaccinated and challenged mouse serological responses to RTX, and PT were determined by qualitative ELISA. High-binding 96-well ELISA plates were coated overnight at $4^{\circ} \mathrm{C}$ with $50 \mu \mathrm{l}$ of purified RTX or Pt in PBS at a concentration of $1 \mu \mathrm{g} / \mathrm{mL}$. Purified B. pertussis antigens, were obtained from Dr. Jennifer Maynard. Serum samples from NVNC, PBS, ACV, and WCV serum titers were analyzed for PT, as the RTX group had no PT in vaccine. Samples from NVNC, PBS, WCV, and RTX were analyzed for serum antibody titers to RTX, as ACV would not be expected to have RTX titers because no RTX was included in vaccine. Plates were then washed with PBS + 1\% Tween 20 (PBS-T), then blocked with 5\% milk in PBS-T for 1 hour at room temperature. Sera were diluted to a concentration in the linear dose range for each antigen. Plates were incubated for $2 \mathrm{~h}$ at $37^{\circ} \mathrm{C}$ with serial diluted serum samples from vaccinated groups. Following 3 PBS-T washes, 1:4000 goat anti-mouse IgG-AP (Southern Biotech), secondary antibody was added and incubated $1 \mathrm{~h}$ at $37^{\circ} \mathrm{C}$. Plates were washed, then developed for $30 \mathrm{~min}$ with $100 \mu \mathrm{l}$ p-nitrophenyl phosphate. Colorimetric signal was measured using Spectramax i3 (Molecular Devices) at 450nm. An average of blanks was subtracted from all absorbances and used as a baseline detection limit. The minimum detection limit above the baseline was analyzed by oneway ANOVA, with a Tukey's multiple-comparison test for each time-point using Prism 7.

\section{Isolation of Lung RNA, illumina library preparation, and sequencing}

Lung RNA was prepared similar to previously reported ${ }^{62}$ with the following modifications. The freshly isolated NECre luc mice lungs were homogenized and RNA was prepared immediately using RNeasy purification kit (Qiagen). Each lung was placed in $1 \mathrm{ml}$ of sterile PBS and then $2 \mathrm{ml}$ of TE lysozyme $(1 \mathrm{mg} / \mathrm{ml})$ was added and allowed to incubate for $10 \mathrm{~min}$ on ice. $2 \mathrm{ml}$ of RLT buffer was added and incubated for 
another $10 \mathrm{~min}$ on ice. The homogenate was then pushed through a syringe needle. The homogenates were pelleted by centrifugation at 20,800 (max speed microfuge) for $10 \mathrm{~min}$. The supernatant was extracted and then $2.8 \mathrm{ml}$ of $100 \% \mathrm{EtOH}$ was added to each tube. This supernatant of each mouse sample was then disturbed to four RNeasy tubes for RNA isolation. The RNA was eluted and pooled into one sample per mouse. The resulting RNA was quantified on a Qubit 3.0 (ThermoFisher) with the high intensity assay kit. Next, the RNA integrity was assessed using Agilent BioAnalyzer RNA Pico chip. All samples were then submitted Ribo-zero rRNA depletion (illumina) and reassessed for RNA integrity. rRNA depleted mRNA samples were then fragmented and prepared into libraries using illumina ScriptSeq Complete Gold (Epidemiology). Libraries were checked for quality control with KAPA qPCR QC assay (KAPA Biosystems). The libraries (33 total) were then sequenced on an illumina HiSeq at the Marshall University Genomics Core facility on 2 lanes of 2x50B. pertussis. Sequencing data were deposited to the Sequence Read Archive (SRA) and are available under the reference number SRA587785, BioProject number PPJNA394758.

\section{RNAseq bioinformatics analyses}

The reads were analyzed using the software CLC Genomics workbench 9.5. Mus musculus genome was downloaded from NCBI (version GRCm38.78). Reads were mapped against the genome using the following settings for mapping: mismatch cost $=$ 2 , insertion cost $=3$, deletion cost $=3$, length fraction $=0.8$, similarity fraction $=0.8$. RPKM values were generated using default parameters for CLC Genomics. On average $\sim 20$ million reads were obtained for each sample and with stringent parameters $\sim 73 \%$ mapped to the murine genome with our mapping parameters. Fold changes in gene expression and statistical analyses were performed using an Extraction of Differential Gene Expression (EDGE) test $p$ value. Expression data for each gene was considered significant if the $p$ value was less than 0.05 . Venn diagrams were generated using Venny $2.1^{63}$. Fold-change of gene expression data was plotted relative to nonchallenged control groups at each time-point. Supplementary Table 8 contains the exported gene expression analysis worksheets and statistical analyses. Gene list were created using GO terms acquired though AmiGO 2 database including neutrophil 
(CL:000075), neutrophil activation (GO:0042119), neutrophil migration (GO:1990266), innate immune response (GO: 0045087), T-helper 1 type immune response (GO:0042088), type 2 immune response (GO:0042092), and T-helper 17 type immune response (GO:0072538) ${ }^{64}$.

\section{Pathway Enrichment Analysis}

Ingenuity Pathway Analysis (Qiagen) was utilized to map lung transcriptomes to biological and disease functions. Lung transcriptomes were loaded to IPA, and comparative analyses were performed at early and late time-points. Gene expression fold-changes were mapped to higher-order disease and function defined by the IPA knowledge base, and a gene enrichment analysis was performed on early and late timepoints. A Fisher Exact T-test was used to determine statistically significant $(p<0.05) p$ values of the functions that made up the higher-order disease category and represented as a range of $p$-values. The relative fold-change of these identified genes was determined, and the 10 highest fold-changes were represented as heat maps.

\section{Immunoglobulin and T-cell receptor profiling}

B cell clones were identified using MiXCR software (MiLabratory), capable of generating quantitated clonotypes of immunoglobulins ${ }^{48}$. The same paired-end Illumina sequenced reads mentioned above were merged using concatenation, then imported into MiXCR software. Merged reads were aligned to each other to generate clonotypes of based on VDJ segment regions of unique immunoglobins specific to each sample. Clone data for each sample was grouped based on vaccine received, and time-point. Clonotypes from each sample were then separated into T and B cells, based on T-cell receptor or B-cell receptor specific sequences. Prepared data files were then imported into VDJtools (MiLabratory), for data representation according to established protocol ${ }^{65}$. Briefly, data was represented to show $\mathrm{V}$-J diversity, and quantify unique clonotypes using dendrograms and chord diagrams. 


\section{Acknowledgements}

B. pertussis strain UT25 was kindly provided by Dr. Sandra Armstrong (University of Minnesota). The NeCre luc mice were developed by lan Glomski and we thank him for originally providing the mice and developing the imaging methodologies. The Elane mice were kindly provided by MRC Harwell (Oxfordshire, UK). RTX antigen was graciously purified by Andrea DiVenere, University of Texas at Austin. We would like to thank the following WVU facilities: Genomics Core, Office of Laboratory Animal Resources (OLAR) for support with the murine studies, Animal Models and Imaging (U54 GM104942) for support IVIS imaging, the flow cytometry and single cell (S10 OD016165). We would like to acknowledge Kathy Brundage for flow cytometry support, Sarah McLaughlin for IVIS support and Ryan Percifield for next generation sequencing library preparation. D.T.B. was supported by the WVU HSC Office of Research and Graduate Education, graduate student fellowship from the West Virginia NASA Space Grant Consortium, and the Jennifer Gossling Fellowship. Mackenna Boone was supported by a WV-InBRE summer fellowship. Additional support was provided by NIH/NIAID grant RO1 AI1018000 (E.L.H.) and NIH/NIAID grant RO1 A1122753 (J.A.M). This work was also supported by funding from National Institutes of Health HHSN272201200005C-416476 and laboratory startup funds from West Virginia University to F.H.D. The Marshall University CORE facilities and RNA sequencing were funded by the WV InBRE grant P20 GM103434.

\section{Author contributions}

All authors participated in the composition and review of the manuscript. D.T.B. designed experiments, performed IVIS imaging, raised and dissected mice, analyzed immunological data, mapped NGS read data and calculated expression analysis. M.E.V. developed flow cytometry panels, and performed analyses. T.W. coordinated murine trials, and prepared RNA for NGS analysis. E.S.N. and E.S.K. analyzed RNAseq and developed visualizations. J.M.H. performed serological analysis. C.E., M.B., S.B., K.D., J.B., and M.E prepared and analyzed samples to measure correlates of protection on each experimental day. J.M. provided the RTX antigen. J.M., E.L.H, M.B. and F.H.D. 
designed the overall strategy of the studies. M.B. and F.H.D. formulated vaccines, directed experiment days, performed dissections, and analyzed RNAseq data.

\section{Competing financial interests}

The authors declare no competing financial interests.

\section{Supplemental Information}

Supplemental information is available under Boehm et al Supplemental Information. 


\section{Figures}

Figure 1: Experimental workflow for vaccination, B. pertussis challenge, sample acquisition, and analysis of this study.

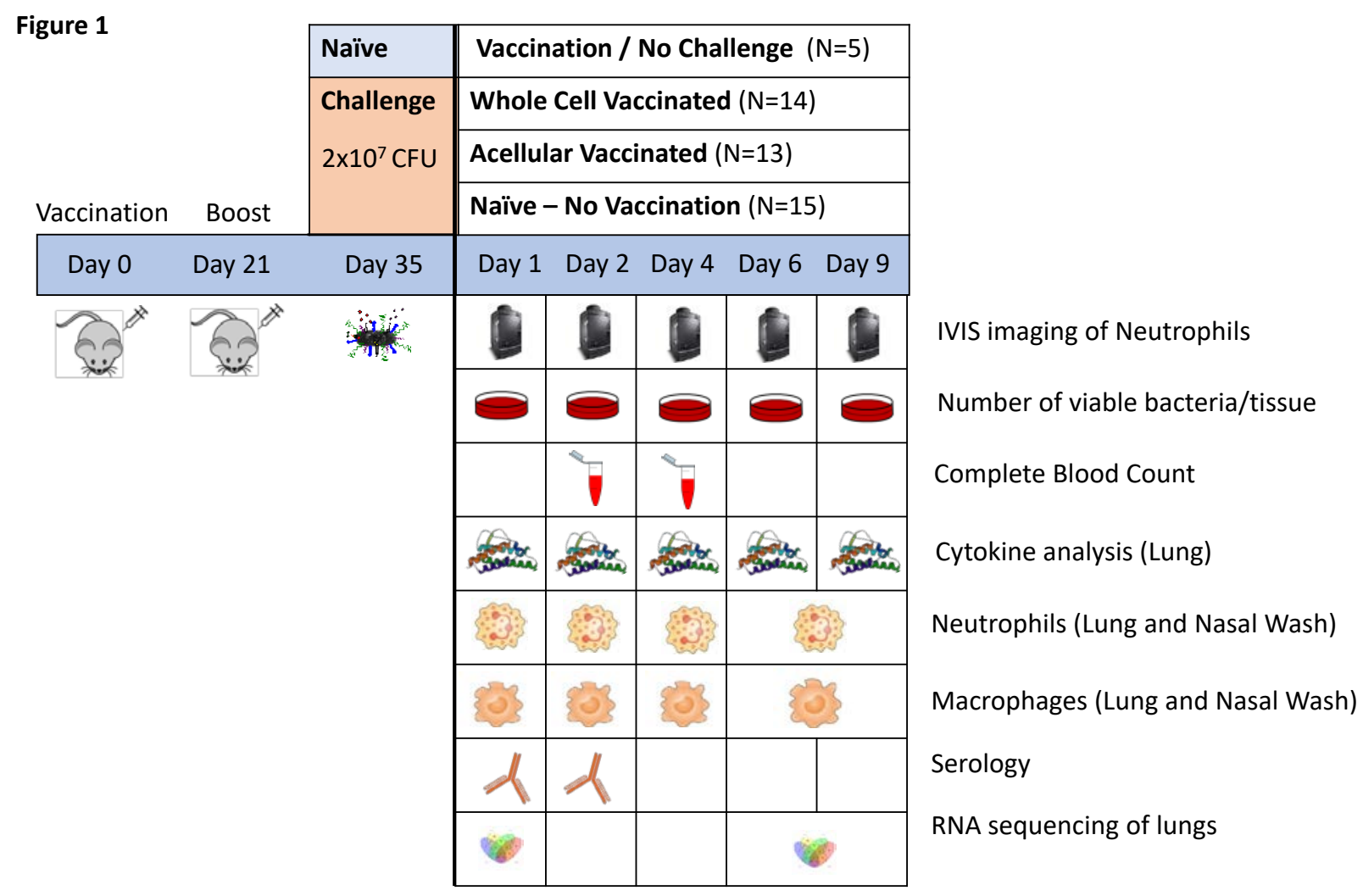

Figure 1: Experimental workflow for vaccination, B. pertussis challenge, sample acquisition, and analysis of this study. Schematic diagram of experimental design showing vaccination schedule, immunization groups, $B$. pertussis challenge, euthanasia, sampling, and analysis method on days 1,2,4, 6 , and 9 days pc in NECre luc mouse model. Mice were initially vaccinated and then given a booster dose of the same vaccine at day 21 . A challenge dose of $2 \times 10^{7}$ viable bacteria was administered by intranasal inhalation at day 35. IVIS imaging of neutrophils was monitored throughout study. The overall numbers of mice per group are indicated. At time-points shown bacterial burden, complete blood cell counts, lung cytokine profiles, lung and nasal wash neutrophil cells, serum antibody titers, and lung transcriptome were determined. To characterize the effects of vaccination, the vaccinated groups were compared to a group of not vaccinated and not challenged controls. 
Figure 2: Kaplan- Meier survival curves of NECre luc mice according to immunization group.

\section{Survival Curve}

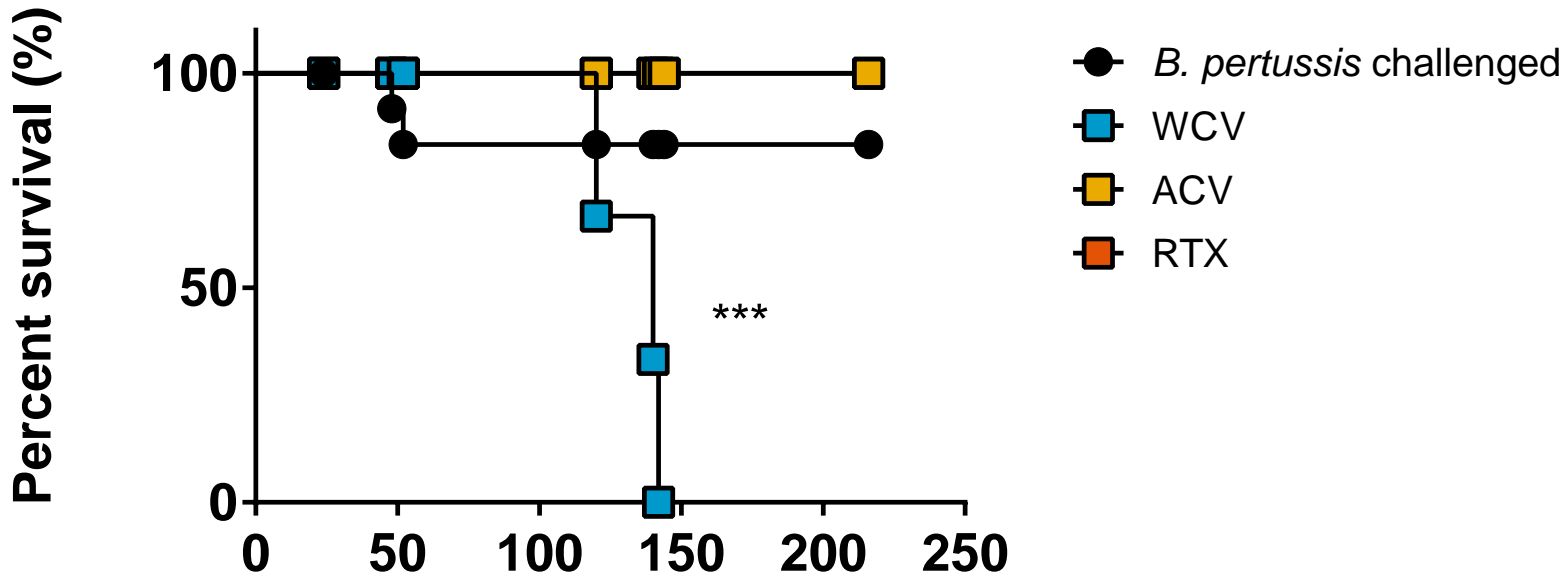

Hours post-infection

Figure 2: Kaplan- Meier survival curves of NECre luc mice according to immunization group. The survival percentage of remaining NECre luc mice (prior to scheduled euthanasia) immunized with WCV, ACV, RTX, and B. pertussis challenged, or not vaccinated and challenged with $B$. pertussis. Log-rank (Mantel-Cox) test: ${ }^{\star \star \star} p<$ 0.0005. In Fig. 1 the time-points where mice were euthanized for analysis are indicated. In this survival curve, we are only showing the mice became morbid throughout the study timeframe. 
Figure 3: Bacterial burden in respiratory tissue and serological responses to $B$. pertussis challenge of immunized and naïve NECre luc mice.
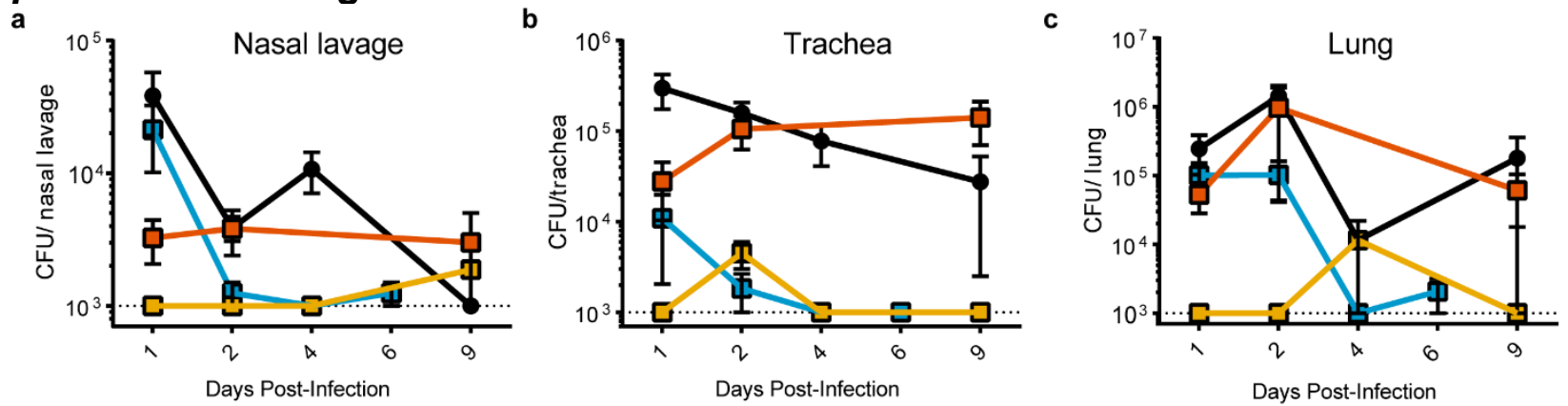

d PT Antigen
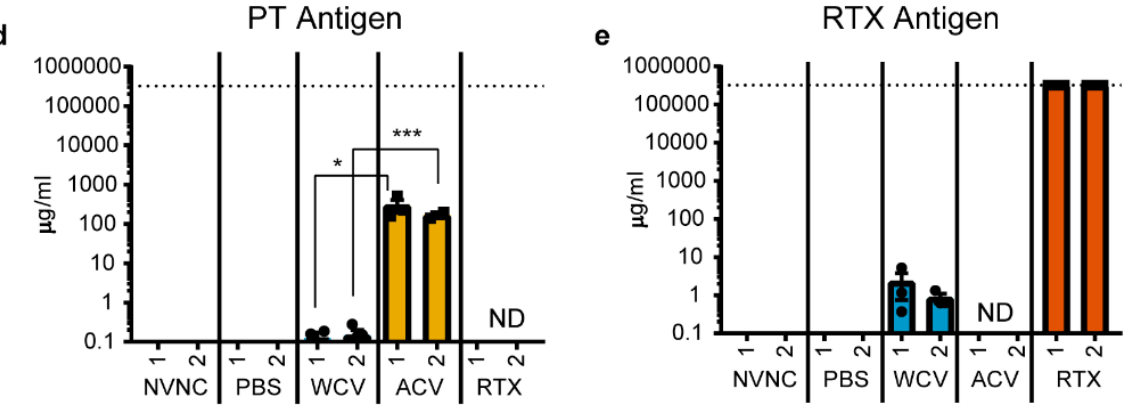

- Naive

D WCV

$\square$ ACV

口 RTX

O NVNC

Figure 3: Bacterial burden in respiratory tissue and serological responses to $B$. pertussis challenge of immunized and naïve NECre luc mice. Mice were vaccinated with PBS control vehicle, WCV, ACV, or RTX then B. pertussis challenged. At days $1,2,4,6$ and $9 \mathrm{pc}$ the bacterial burdens were determined by culturing of $(A)$ nasal lavage, homogenates of (B) trachea and (C) lung on BG agar. The dashed line at 1000 CFUs represents the lower limit of detection, due to plating. Data in each group were compared to PBS control using an unpaired two-tailed t-test. Significant differences are not indicated on the graphs for clarity (Supplementary Table 2 indicates all statistics performed). 
Figure 4: IVIS imaging and flow cytometric analysis of naïve and immunized NECre luc mice post challenge with $B$. pertussis.

a

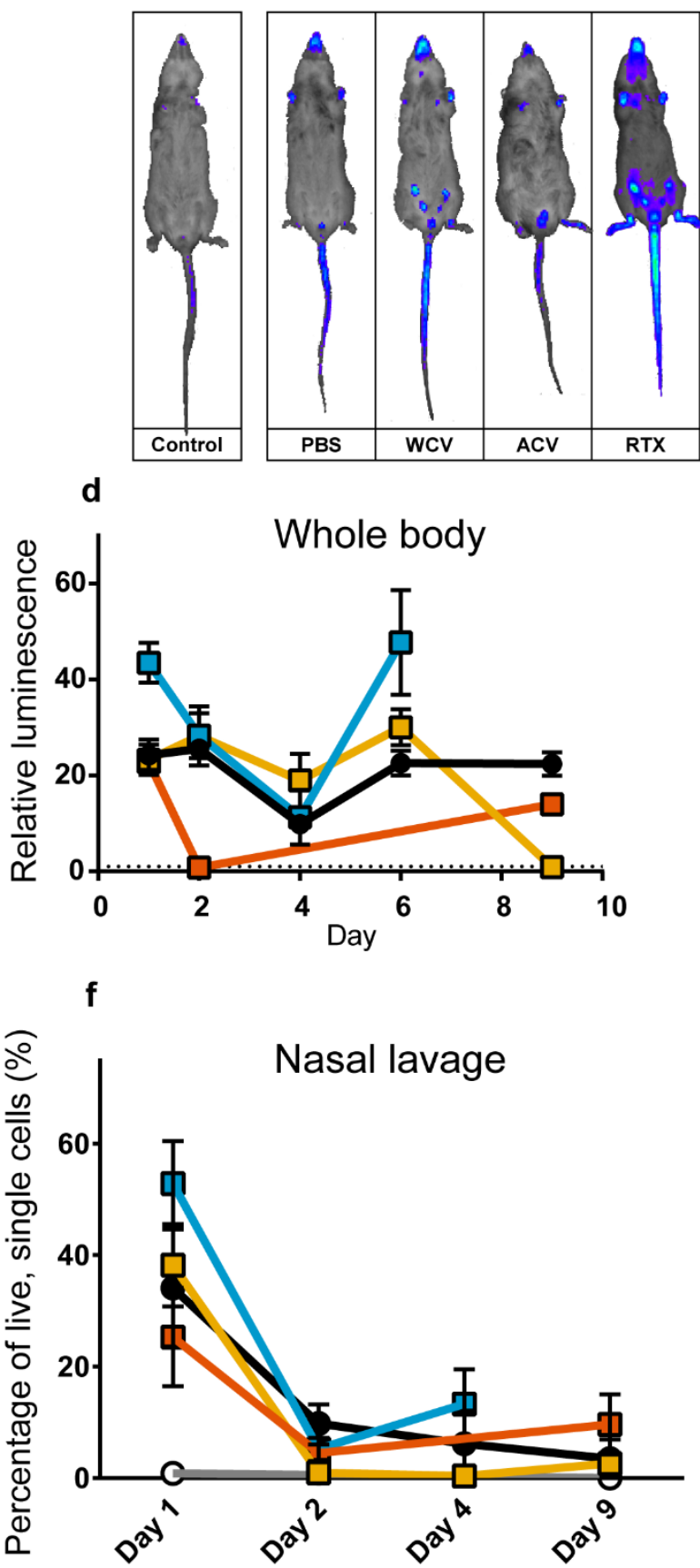

b

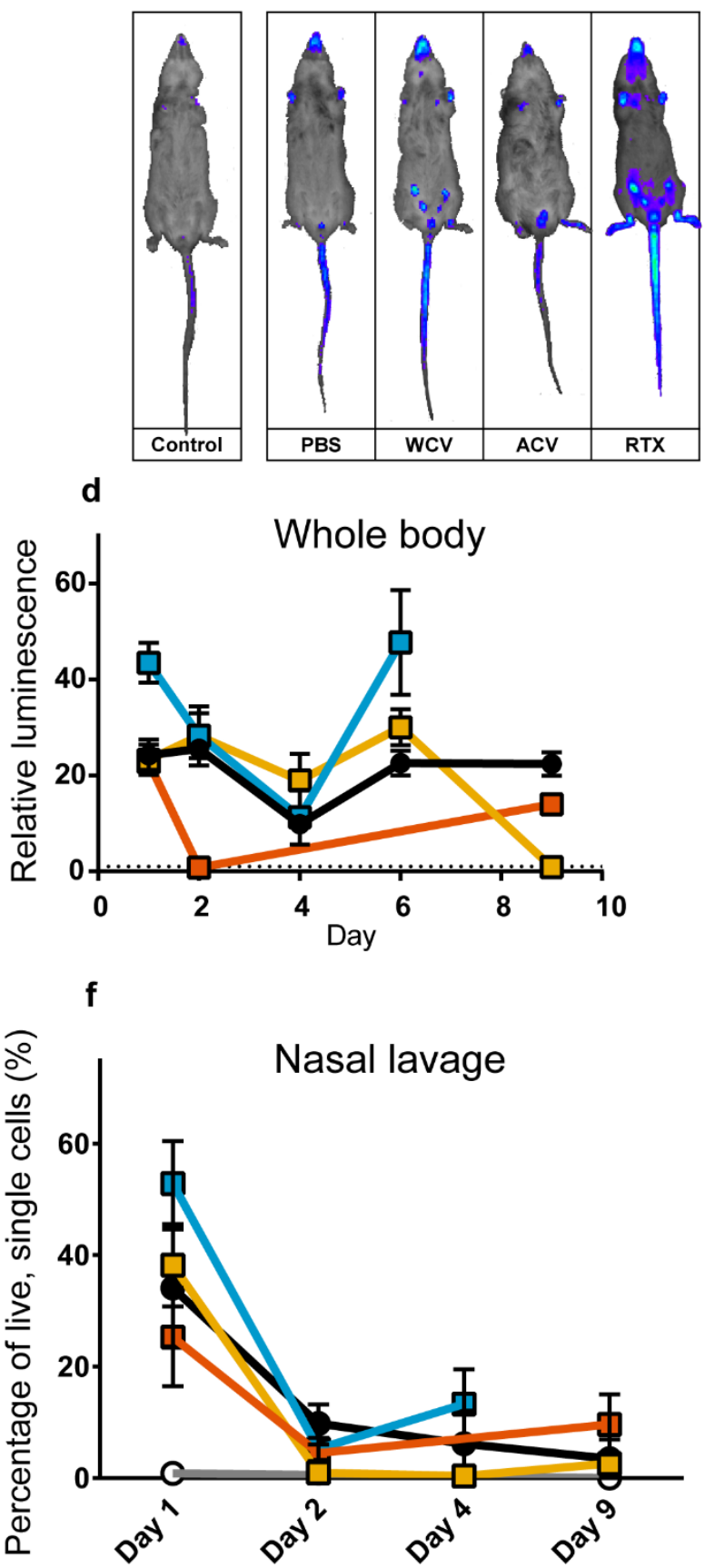

d

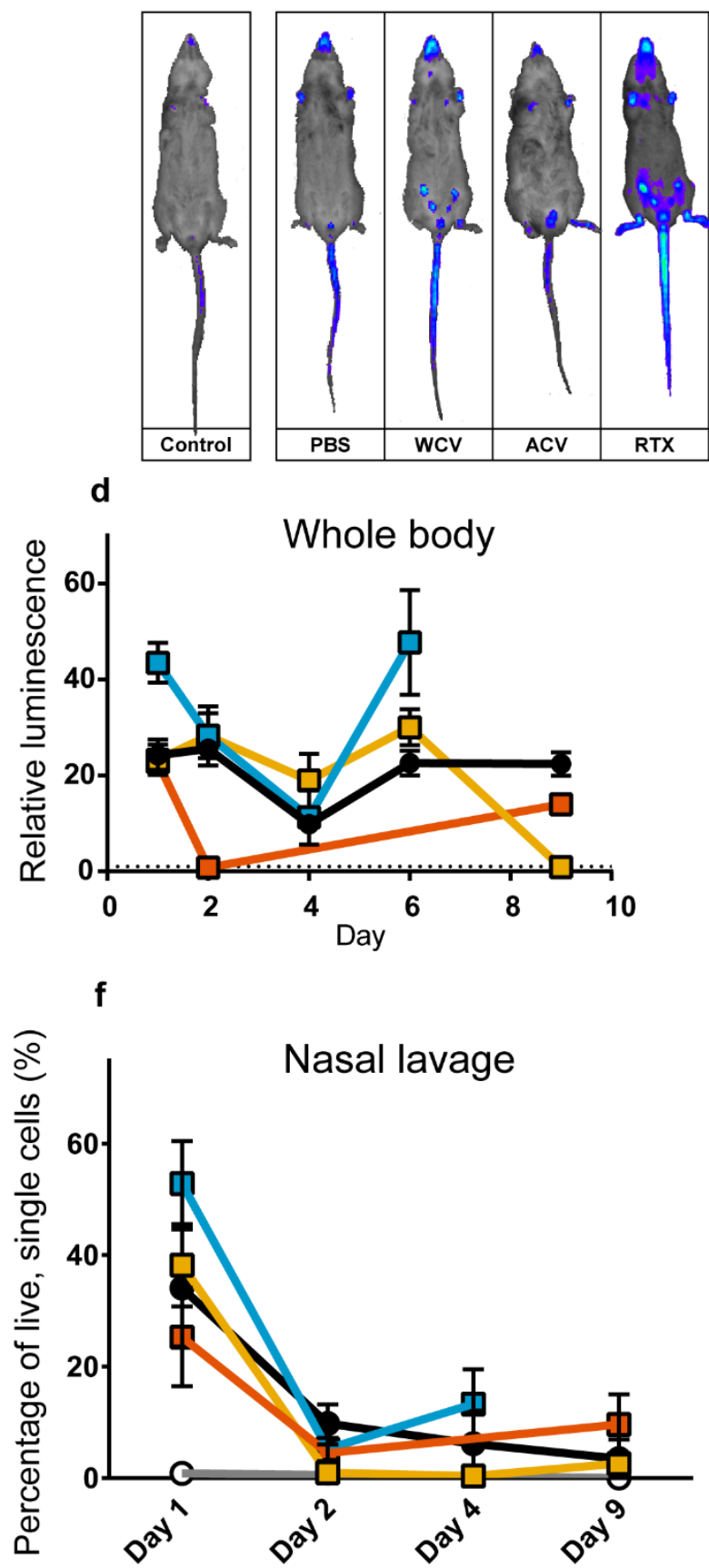

f

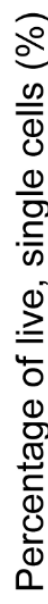

Day 1 c Day 6
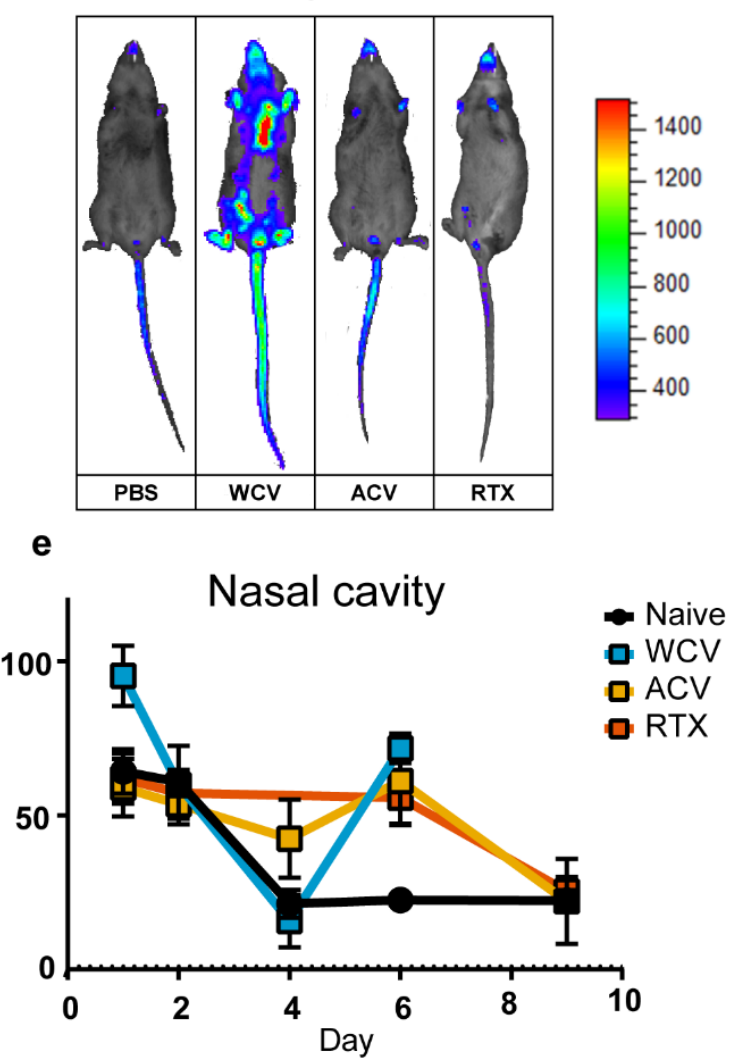

g

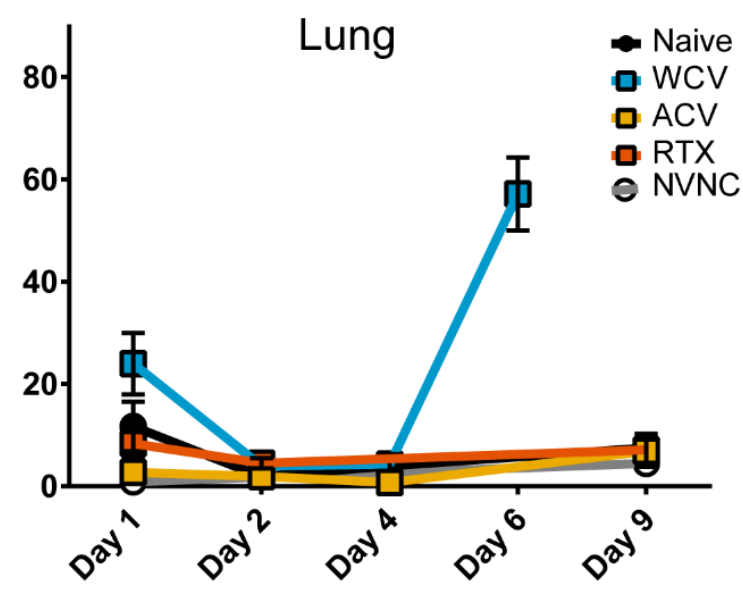

Figure 4: IVIS imaging and flow cytometric analysis of naïve and immunized NECre luc mice post challenge with $B$. pertussis. IVIS imaging of luminescent neutrophils in anesthetized mice following luciferin injection. (A) Representative images of naïve, not $B$. pertussis challenged NECre luc mice. (B) Representative images of 
PBS control, WCV, ACV, and RTX immunized and B. pertussis challenged NECre luc mice at day $1 \mathrm{pc}$ and $(C)$ day $6 \mathrm{pc}(\mathrm{D}$ and $\mathrm{E})$. Luminescence was measured on Xenogen IVIS Lumina II. Relative luminescence levels quantified by fold change of emitted photons/second of NECre luc mice following $B$. pertussis challenge $(\mathrm{N}=3-5)$ compared to average luminescence of naïve, not challenged NECre luc mice $(N=5)$. Neutrophil luminescence of $(D)$ whole animal signal and $(E)$ nasal cavity was determined at days $1,2,4,6$, and 9 pc. (f) Quantification of the percentage of live, single cells classified as neutrophils (GR-1+CD11b+) detected in nasal lavage (f) and lung homogenate $(g)$. Significant differences are not indicated on the graphs for clarity, but are included in Supplementary Table 3. 
Figure 5: Analysis of cytokine profiles from lungs of naïve and immunized NECre luc mice post challenge with $B$. pertussis.

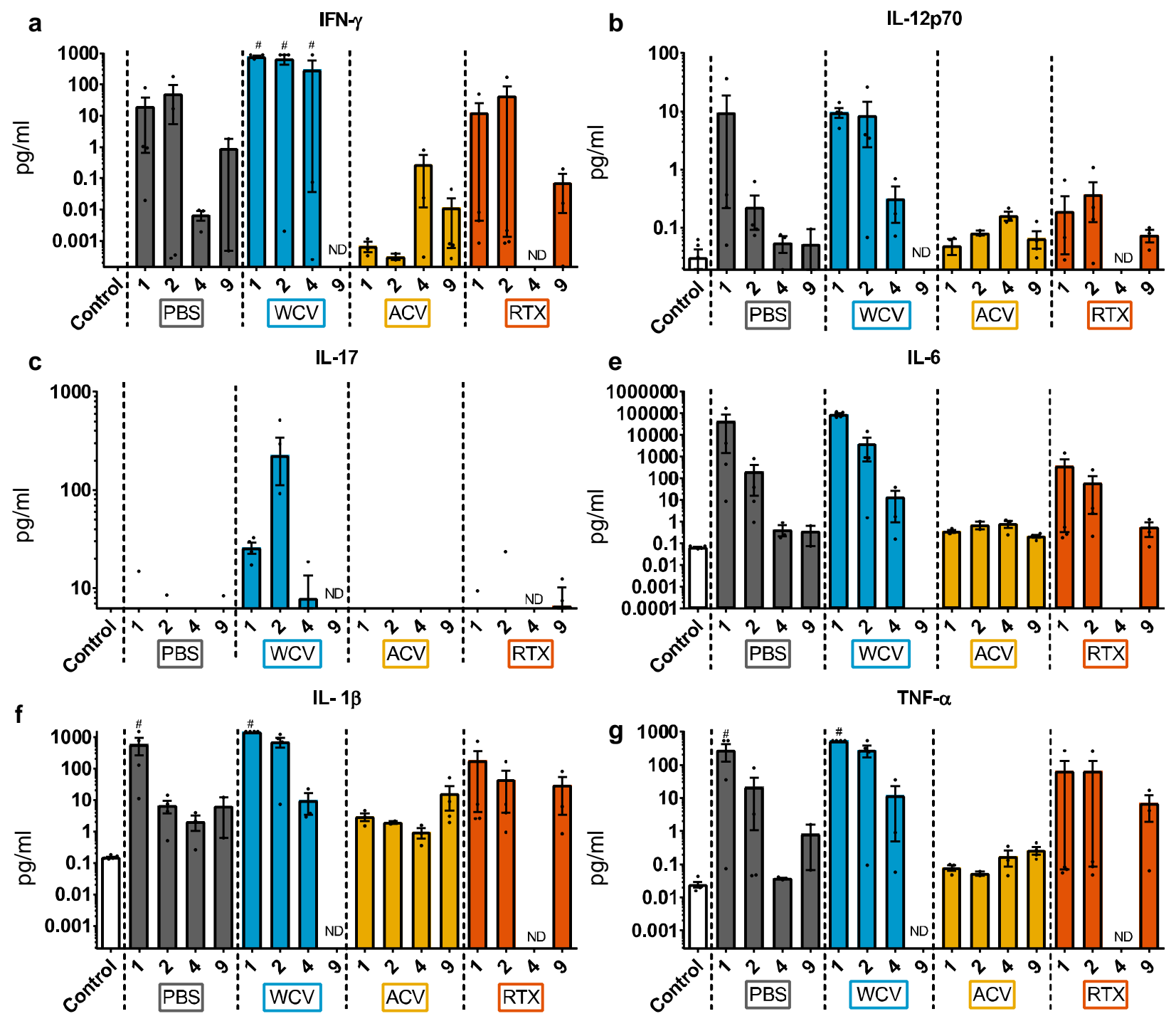

Figure 5: Analysis of cytokine profiles from lungs of naïve and immunized NECre luc mice post challenge with $\boldsymbol{B}$. pertussis. Th1 associated cytokines from the supernatant of lung homogenates were analyzed at days 1,2,4 and 9 pc. Cytokines (a) IFN- $\gamma$, (b) IL-12p70, (c) IL-17, (d) IL-6, (e) IL-1ß, (f) TNF- $\alpha$ were quantified using electrochemiluminescence immunoassays. Significant differences are not indicated on the graphs for clarity but, are included in Supplementary Table 4. ND: Sample not determine, \#: data above upper limits of detection. 
Figure 6: Lung transcriptome profile of vaccinated and challenged NECre luc mice.

\section{EARLY}

a

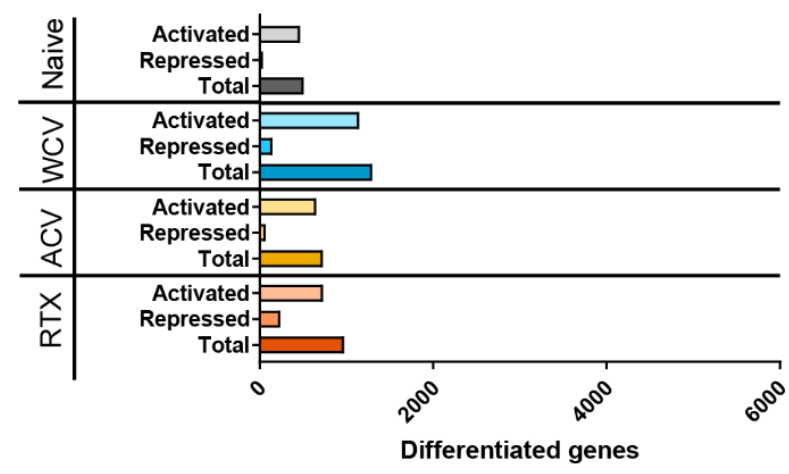

b

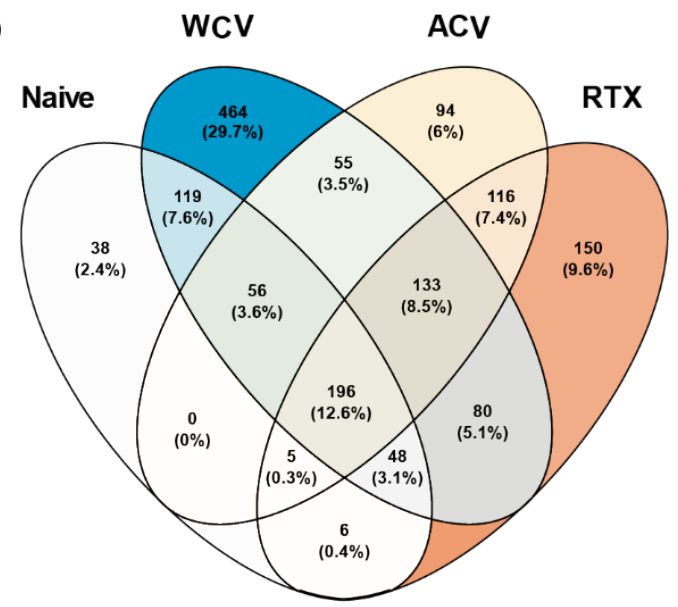

LATE
C

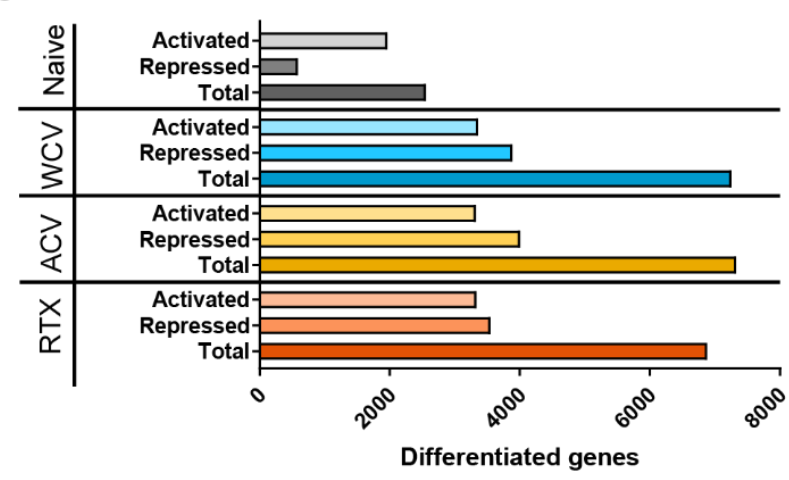

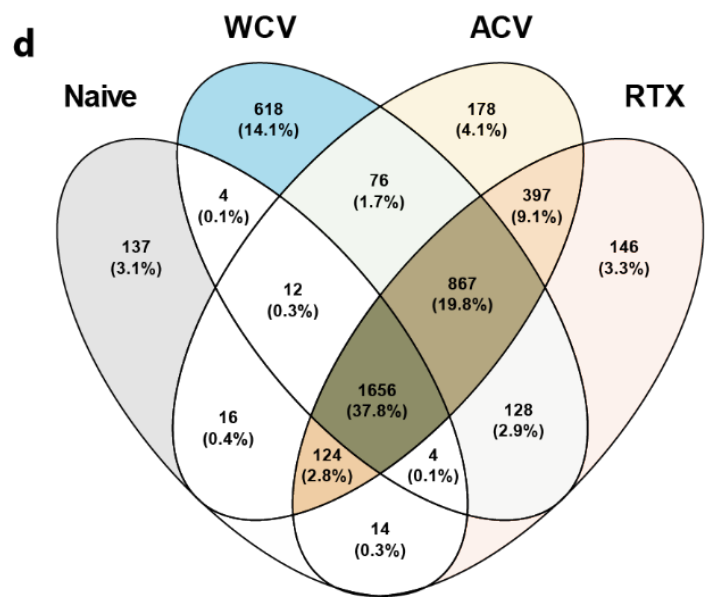

Figure 6: Lung transcriptome profile of vaccinated and challenged NECre luc mice. RNA sequencing was performed on total RNA from homogenized lung on days 1 (Early) or 6/9 (Late) following challenge with B. pertussis. (a) The total number of statically differentiated genes found in lung transcriptome at early (a) or late (c) timepoints. Statistically differentiated genes were categorized as those that were activated or repressed. Venn diagram of statistically differentiated genes either unique or common to vaccinated or naïve groups at early (b) or late (d) time-points. 
Figure 7: Enrichment analysis and relative fold-changes of genes upregulated following $B$. pertussis challenge.
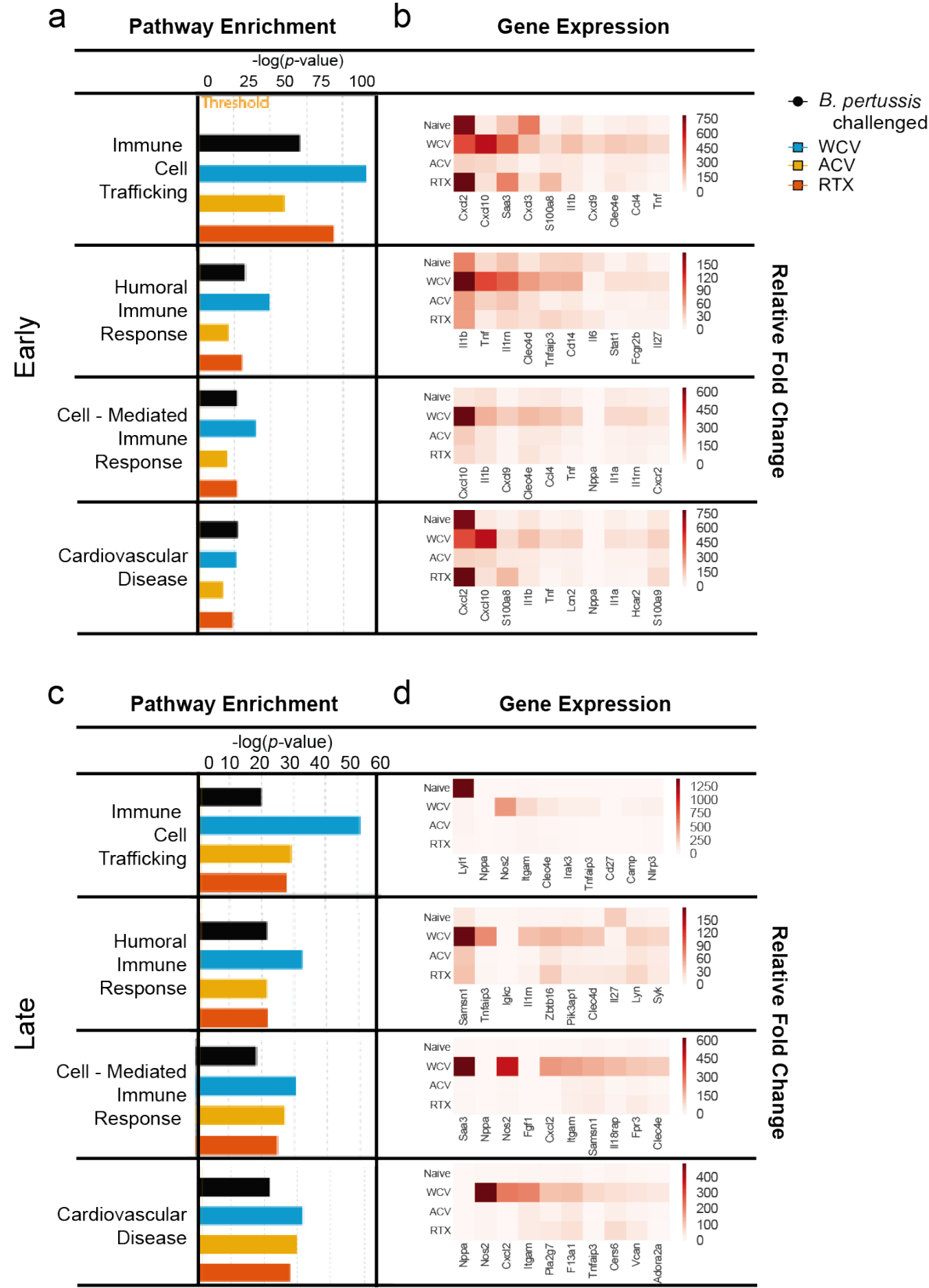

Figure 7: Enrichment analysis and relative fold-changes of genes upregulated following $\boldsymbol{B}$. pertussis challenge. Early (day 1)(a), and late (day 9)(b) IPA comparative analysis of lung transcriptomes from vaccinated and non- 
vaccinated/challenged groups was performed, gene enrichment $p$-values are represented in -log scale. $P$-value was calculated by IPA software based on the number of genes found in a certain data set to the total number of genes associated to a particular function in the IPA knowledge base. Threshold indicates the significance $(p<0.05$, Fisher's exact t-test) Black-PBS, Blue - WCV, Yellow-ACV, Red - RTX. Relative fold changes of ten highest genes associated with a particular function annotation present in either of the experimental groups. 
Fig. 8: Differentiated genes associated with neutrophil recruitment at early and late time-points following $B$. pertussis challenge.

a

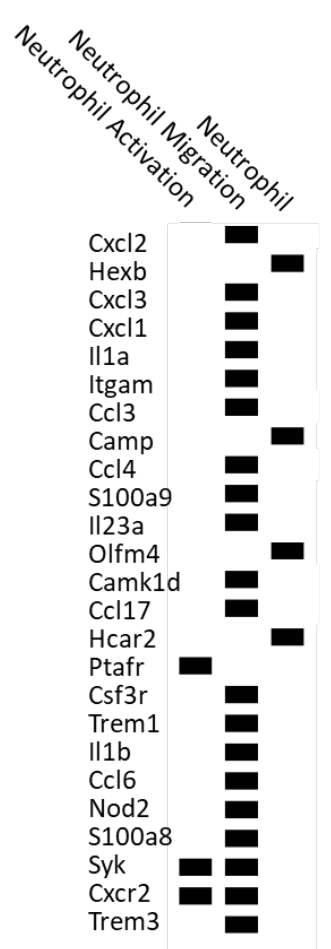

b

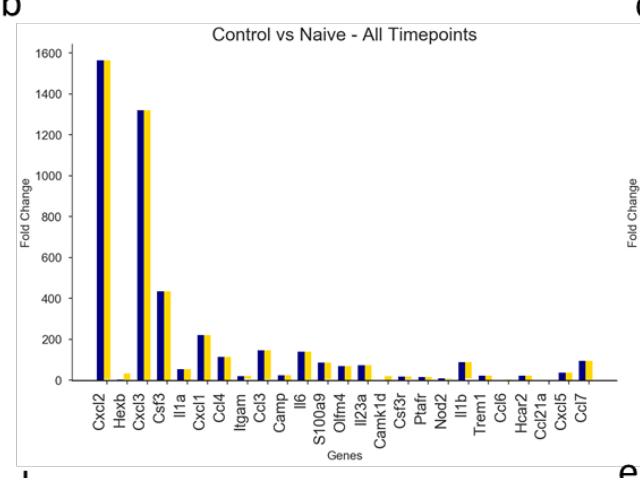

d

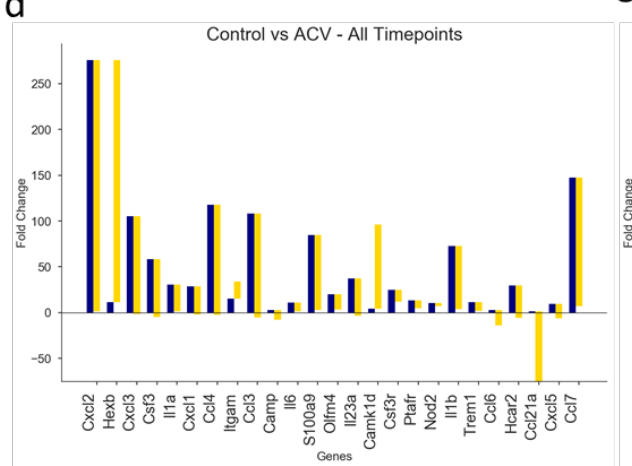

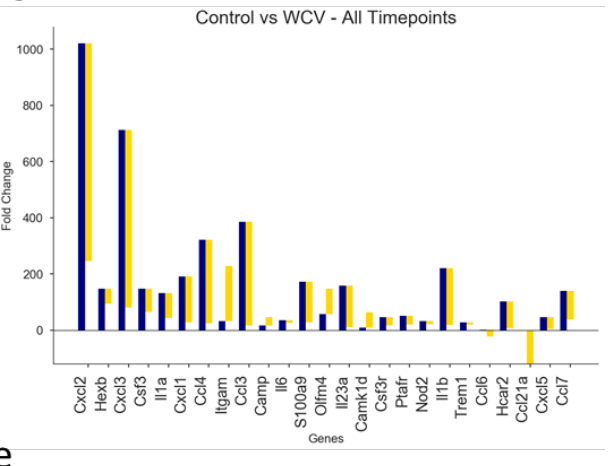

e

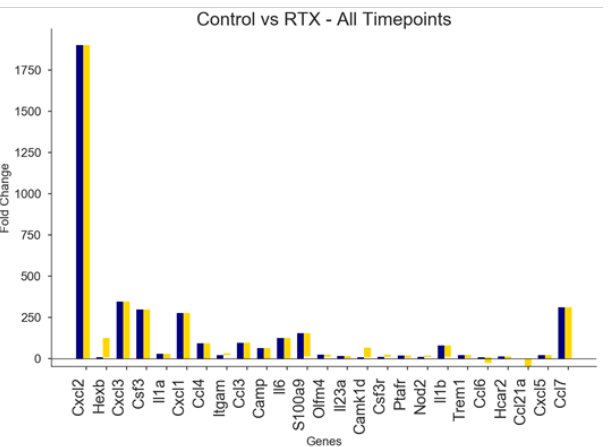

Fig. 8: Differentiated genes associated with neutrophil recruitment at early and late time-points following $B$. pertussis challenge. Gene expression profiles are shown for (a) Naïve, (b) WCV, (c) ACV, and (d) RTX immunized and challenged mice. Blue and gold bars indicate the fold change compared to control non-challenge nonvaccinated mice at early and late time-points, respectively. Genes shown are the 25 genes with the highest variance across all groups between early and late time-point. Genes were ranked based on variance in fold change across groups for the early and late time-points, separately. 


\section{Supplemental Information}

Characterizing the innate and adaptive responses of immunized mice to Bordetella pertussis infection using in vivo imaging and transcriptomic analyses

Dylan T. Boehm ${ }^{\mathrm{a}}$, Melinda E. Varneya, Ting Y. Wonga, Evan S. Nowak ${ }^{\mathrm{a}}$, Emel SenKilic $^{a}$, Jesse Halla ${ }^{a}$ Shelby D. Bradford ${ }^{a}$, Katherine DeRoos ${ }^{a}$, Justin Bevere ${ }^{a}$, Matthew Epperly $^{a}$, Jennifer A. Maynard ${ }^{b}$, Erik L. Hewlett ${ }^{c}$, Mariette Barbiera, and F. Heath Damron $^{\mathrm{a}^{*}}$

a Department of Microbiology, Immunology, and Cell Biology, West Virginia University, Morgantown, WV, USA

${ }^{\mathrm{b}}$ Department of Chemical Engineering, University of Texas at Austin, Austin, TX 78712, USA

c Department of Medicine, Division of Infectious Diseases and International Health, University of Virginia, Charlottesville, Virginia, USA 
Supplementary Fig. 1: IVIS imaging of naïve and immunized NECre luc mice post challenge with $\mathrm{Bp}$ as image was acquired from Xenogen IVIS Lumina II.

a
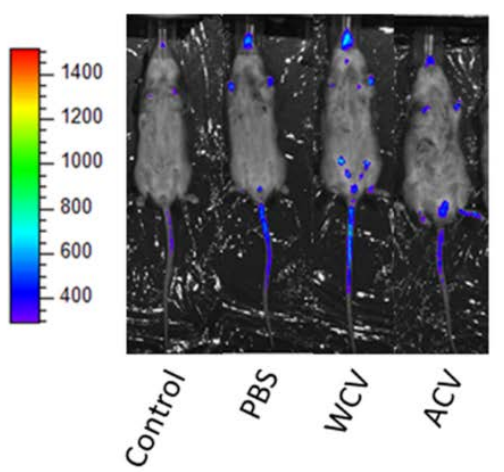

C

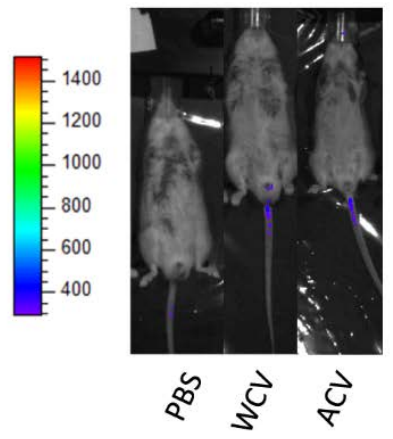

b

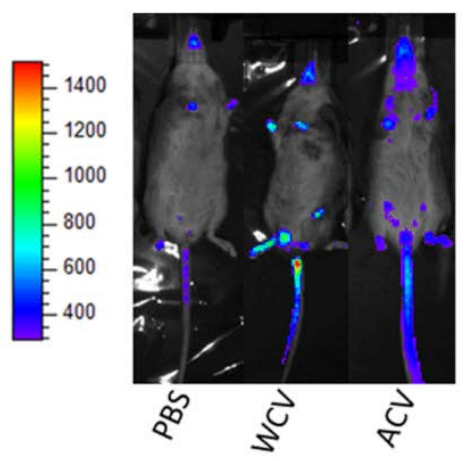

d

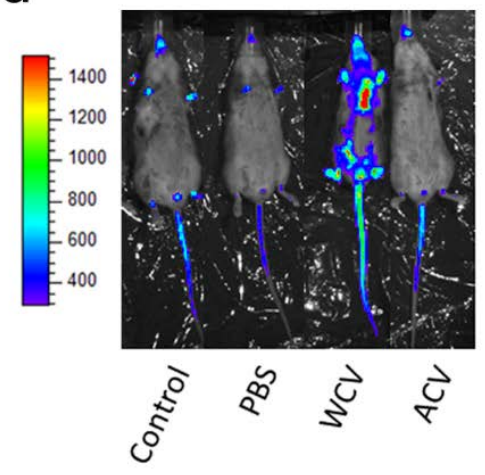

Supplementary Fig. 1: IVIS imaging of naïve and immunized NECre luc mice post challenge with $B p$ as image was acquired from Xenogen IVIS Lumina II. Images at day (a) 1, (b) 2, (c) 4, and (d) 6 - WCV or 9 - Control (Non-vaccinated/ no challenge), PBS, and ACV. Relative luminescence was quantified based on region of interest luminescence in these non-adjusted images. 
Supplementary Table 1. Compositions of vaccines of this study

\begin{tabular}{|l|c|c|c|}
\hline & \multicolumn{3}{|c|}{ Vaccine groups } \\
\hline Vaccine component $^{*}$ & $\mathbf{a P}_{\left(\mathbf{1} 5^{\text {th }}\right.}$ human dose $)$ & WP P**$^{* *}$ & $\mathbf{R T X}+$ alum \\
\hline Pertussis Toxoid $^{\star \star *}$ & 5 & 0.4 & 0 \\
\hline Filamentous Hemagglutinin & 5 & 3.5 & 0 \\
\hline Pertactin & 1.6 & 0.3 & 0 \\
\hline RTX & 0 & 0.2 & 10 \\
\hline Aluminum hydroxide & 125 & 0 & $1(\mathrm{mg})$ \\
\hline Other antigens/adjuvants & 0 & 62 & 0 \\
\hline
\end{tabular}

*All masses of antigens or adjuvant are indicated in $\mu \mathrm{g}$

** This estimate is based on the number of peptides identified for each antigen in the WP. The percentage is then used to estimate the potential mass based on the fact that the wP dose used in this study contained $66 \mu \mathrm{g}$ of total protein.

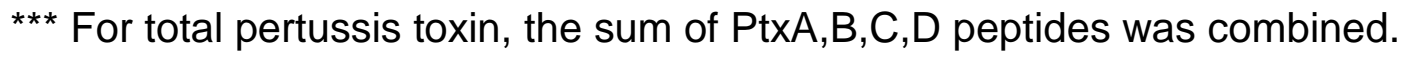


Supplementary Table 2. Statistical analysis from Figure 3abc.

\begin{tabular}{|c|l|r|l|r|l|r|}
\hline & Day 1 & Day 2 & Day 4 & \\
\hline \multirow{3}{*}{ Trachrea } & ACV & 0.0524 & WCV & 0.0341 & & \\
\cline { 2 - 7 } & WCV & 0.059 & & & & \\
\cline { 2 - 7 } & RTX & 0.0729 & & & & \\
\hline $\begin{array}{c}\text { Nasal } \\
\text { Lavage }\end{array}$ & ACV & 0.0984 & ACV & 0.0764 & ACV & 0.0567 \\
\cline { 2 - 6 } & & & WCV & 0.0209 & WCV & 0.0567 \\
\hline
\end{tabular}

Unpaired two-tailed T tests to PBS challenged group 


\section{Supplementary Table 3: Relevant statistical differences in relative fold changes of neutrophil luminescence of Bp challenged mice.}

Luminescence was analyzed by one-way ANOVA with a Tukey's multiple comparison test at each time point.

\begin{tabular}{|c|c|c|c|c|c|c|c|c|c|c|c|c|}
\hline \multirow{8}{*}{ Whole Mouse } & Day 1 & $P$ value & Day 2 & P value & Day 3 & P value & Day 4 & Pvalue & Day 6 & $P$ value & Day 9 & $P$ value \\
\hline & non-infected vs. PBS & 0.0002 & non-infected vs. PBS & 0.0004 & non-infected vs. PBS & 0.002 & non-infected vs. ACV & 0.013 & non-infected vs. WCV & 0.0001 & non-infected vs. PBS & 0.0006 \\
\hline & non-infected vs. WCV & $<0.0001$ & non-infected vs. WCV & 0.0002 & non-infected vs. WCV & $<0.0001$ & & & non-infected vs. ACV & 0.0035 & non-infected vs. RTX & 0.0076 \\
\hline & non-infected vs. ACV & 0.0004 & non-infected vs. ACV & 0.0003 & non-infected vs. ACV & 0.0003 & & & non-infected vs. RTX & 0.0042 & PBS vs. ACV & $<0.0001$ \\
\hline & non-infected vs. RTX & 0.0007 & PBS vs. RTX & 0.0009 & non-infected vs. RTX & 0.0001 & & & & & ACV vs. RTX & 0.0003 \\
\hline & PBS vs. WCV & 0.0007 & WCV vs. RTX & 0.0004 & PBS vs. WCV & 0.01 & & & & & & \\
\hline & WCV vs. ACV & 0.0004 & ACV vs. RTX & 0.0006 & WCV vs. ACV & 0.0102 & & & & & & \\
\hline & WCV vs. RTX & 0.0004 & & & & & & & & & & \\
\hline \multirow{6}{*}{ Nasal Cacity } & Day 1 & P value & Day 2 & $P$ value & Day 3 & P value & Day 4 & P value & Day 6 & $P$ value & Day 9 & $P$ value \\
\hline & non-infected vs. PBS & 0.0002 & non-infected vs. PBS & $<0.0001$ & non-infected vs. PBS & 0.0017 & non-infected vs. ACV & 0.0047 & non-infected vs. PBS & 0.0247 & non-infected vs. PBS & 0.0132 \\
\hline & non-infected vs. WCV & $<0.0001$ & non-infected vs. WCV & $<0.0001$ & non-infected vs. WCV & $<0.0001$ & & & non-infected vs. WCV & 0.0004 & & \\
\hline & non-infected vs. ACV & 0.0006 & non-infected vs. ACV & 0.0005 & non-infected vs. ACV & 0.0004 & & & non-infected vs. ACV & 0.0009 & & \\
\hline & non-infected vs. RTX & 0.0005 & non-infected vs. RTX & 0.0005 & non-infected vs. RTX & 0.0002 & & & non-infected vs. RTX & 0.0038 & & \\
\hline & WCV vs. ACV & 0.0262 & & & WCV vs. ACV & 0.0314 & & & & & & \\
\hline
\end{tabular}


Supplementary Fig. 2: Representative images and gating strategy for neutrophils from lung and nasal lavage tissues.

Lung homogenate

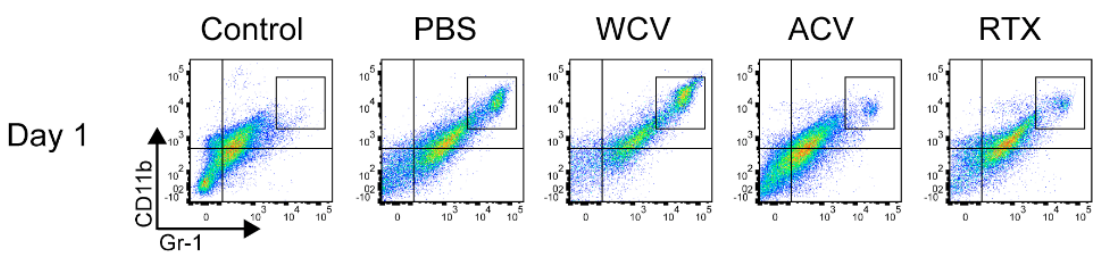

Day 2
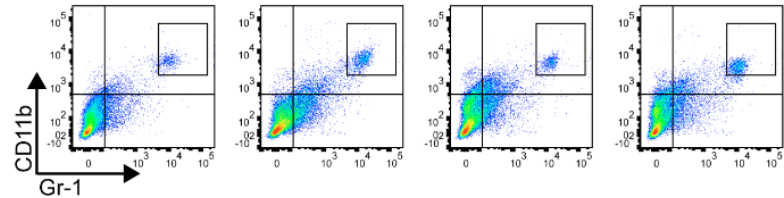

Day 4
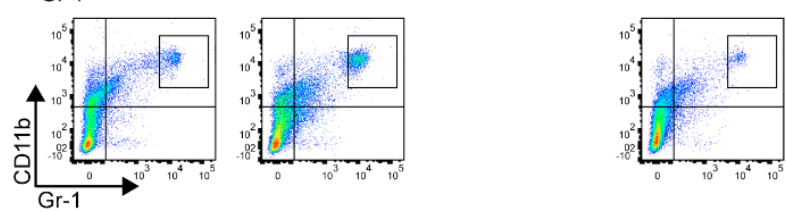

Day 9
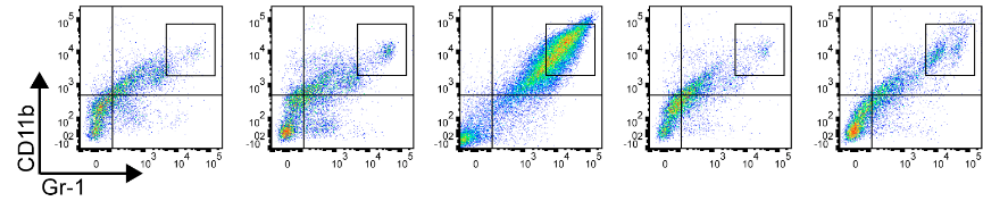

\section{$\underline{\text { Nasal Lavage }}$}

Control

PBS

WCV

ACV

RTX

Day 1
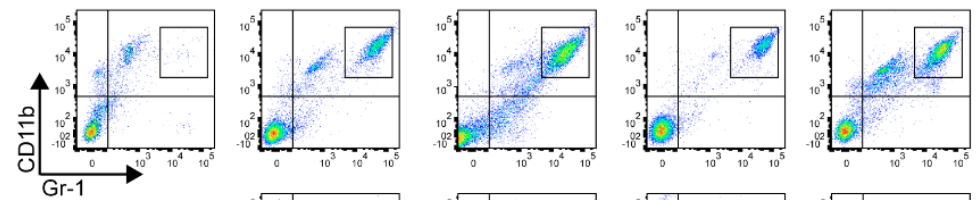

Day 2
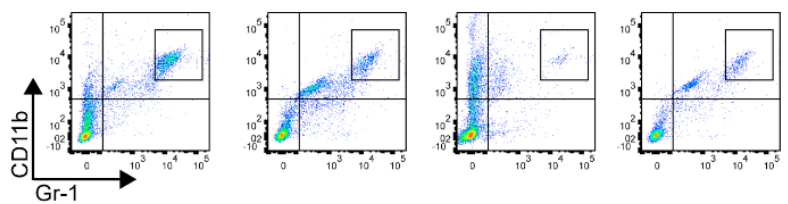

Day 4
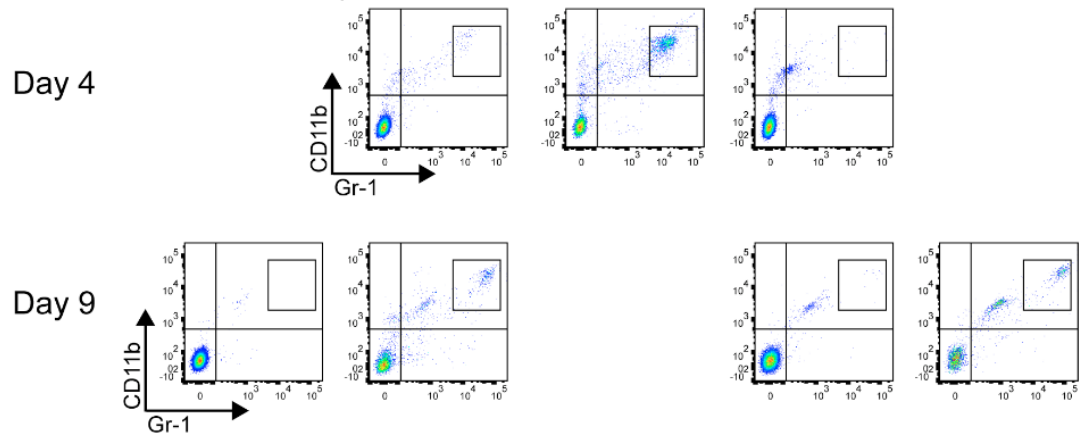
Supplementary Fig. 2: Representative images and gating strategy for neutrophils from lung and nasal lavage tissues. Neutrophils were gated as live, single cells (data not shown), quadrants demonstrate positively labeled cells determined by single marker flow cytometry controls. Region of interest shows neutrophil population $\left(\mathrm{Gr}-1^{+} \mathrm{CD} 11 \mathrm{~b}^{+}\right)$. (a) Lung and (b) nasal lavage representatives. 
Supplementary Fig. 3: Analysis of Treg and Th2 associated cytokines from lungs of naïve and immunized NECre luc mice post challenge with $B$. pertussis

a

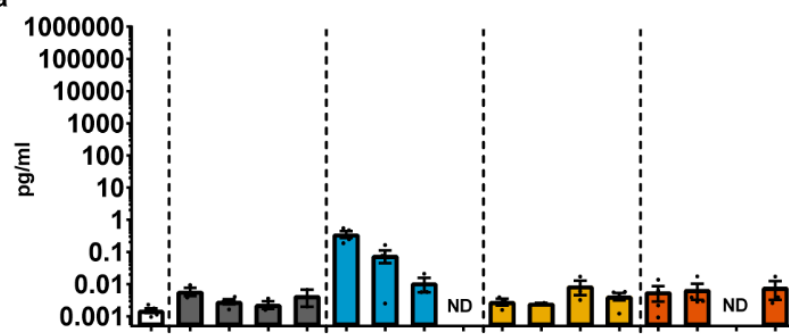

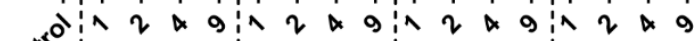

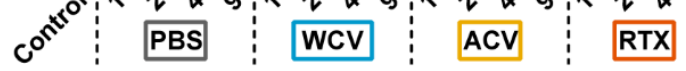

IL- 5

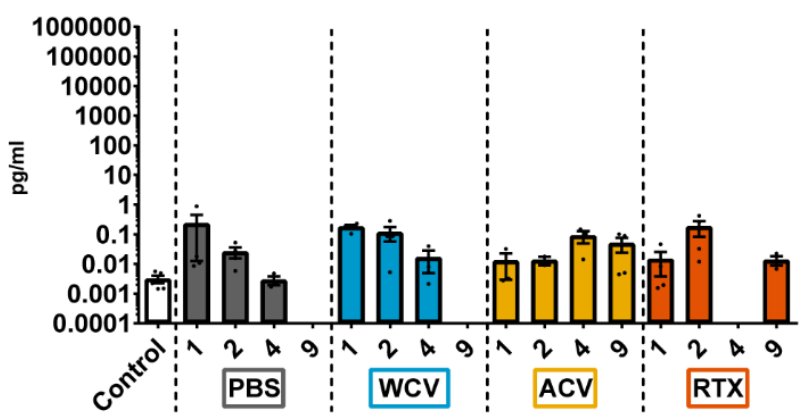

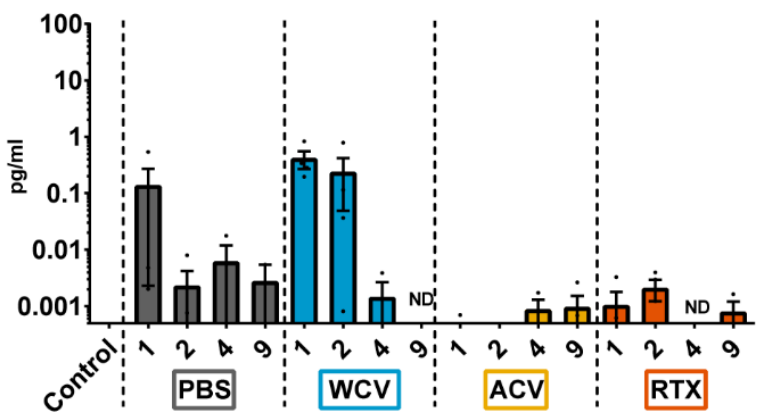

IL- 4

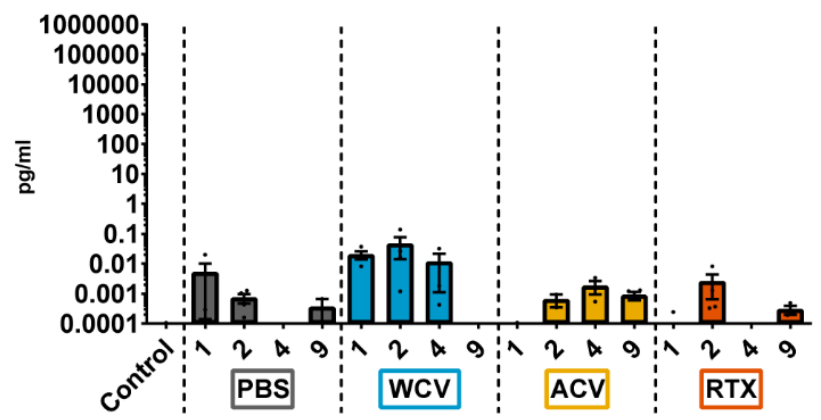

Supplementary Fig. 3: Analysis of Treg and Th2 associated cytokines from lungs of naïve and immunized NECre luc mice post challenge with $B$. pertussis. Cytokines were analyzed at days 1, 2, 4 and 9 pc, then quantified using electrochemiluminescence immunoassays. (a) Cytokines IL-10 and IL-5 associated with Treg immune response. (b) Cytokines IL-2 and IL-4 associated with a Th2 immune response. ND: Sample not determined. 
Supplementary Table 4: Relevant statistical differences between cytokine production in lungs of immunized and $B p$ challenged NECre luc mice.

Samples were analyzed by one-way ANOVA with a Tukey's multiple comparison test at each time point.

\begin{tabular}{|c|c|c|c|c|}
\hline & Day 1 & & Day 2 & \\
\hline & & $P$ value & & $P$ value \\
\hline IL-6 & NV/NC vs. WCV & 0.0251 & & \\
\hline & WCV vs. RTX & 0.0361 & & \\
\hline IFN- - & NV/NC vs. WCV & 0.0175 & NV/NC vs. WCV & 0.0022 \\
\hline & PBS vs. WCV & 0.0261 & PBS vs. WCV & 0.004 \\
\hline & WCV vs. RTX & 0.0408 & WCV vs. ACV & 0.0162 \\
\hline & WCV vs. ACV & 0.0257 & WCV vs. RTX & 0.0039 \\
\hline TNF- $\alpha$ & NVINC vs. WCV & 0.0232 & & \\
\hline & WCV vs. RTX & 0.033 & & \\
\hline $\mid \mathrm{IL}-1 \beta$ & & & NV/NC vs. WCV & 0.0044 \\
\hline & & & PBS vs. WCV & 0.0072 \\
\hline & & & WCV vs. ACV & 0.0288 \\
\hline & & & WCV vs. RTX & 0.011 \\
\hline IL-2 & NV/NC vs. WCV & 0.0224 & & \\
\hline & WCV vs. RTX & 0.0321 & & \\
\hline
\end{tabular}


Supplemental Fig. 4: Volcano plot analysis of differentiated genes at early and late time-points of immunized or naïve mice following challenge.
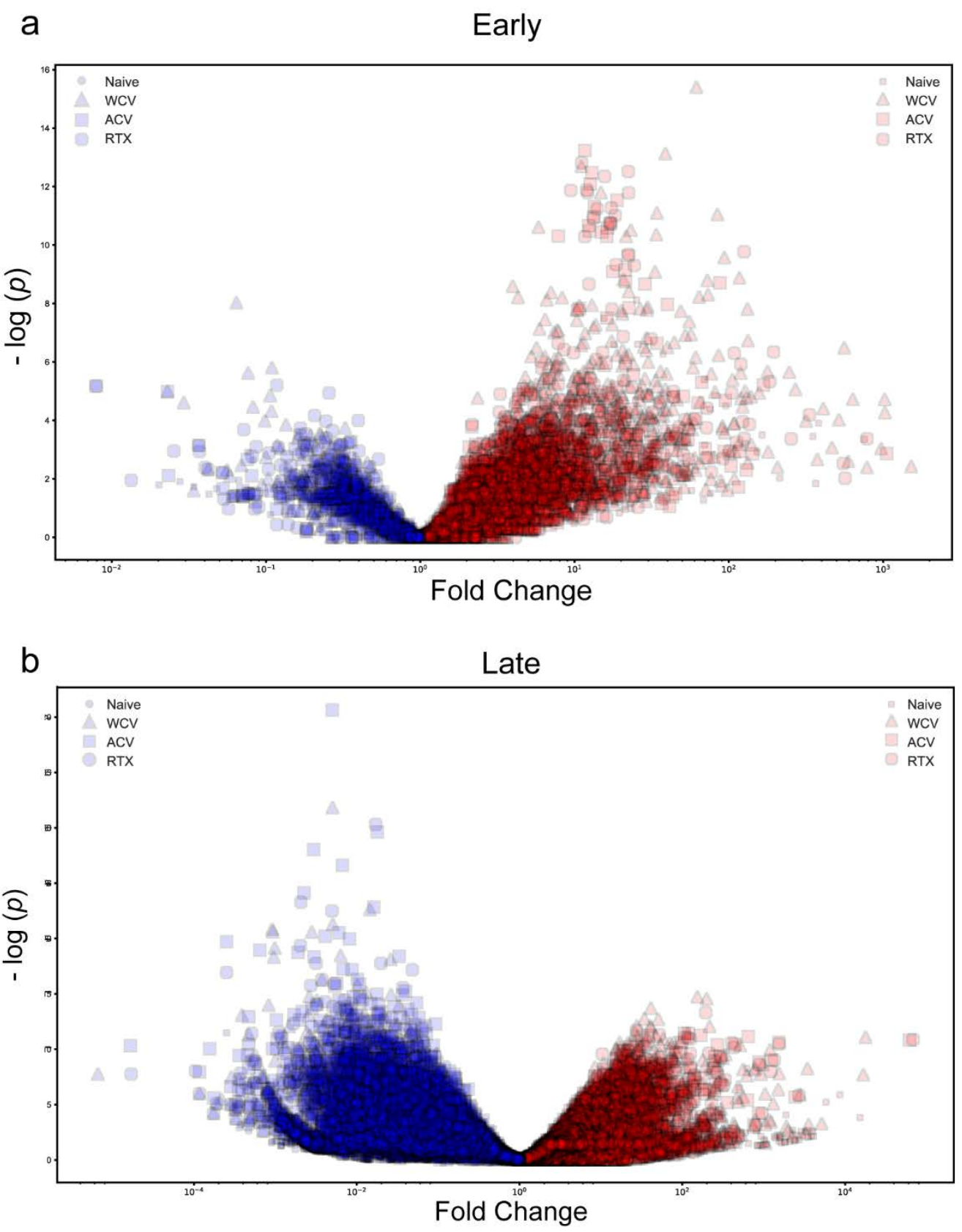

Supplemental Figure 4: Volcano plot analysis of differentiated genes at early and late time-points of immunized or naïve mice following challenge. Upregulated genes are represented in red, repressed gens are in blue. Relative fold change was calculated compared to not infected control mice. 
Supplementary Fig. 5: Gene expression profiles of T helper cell immune responses at early time point.
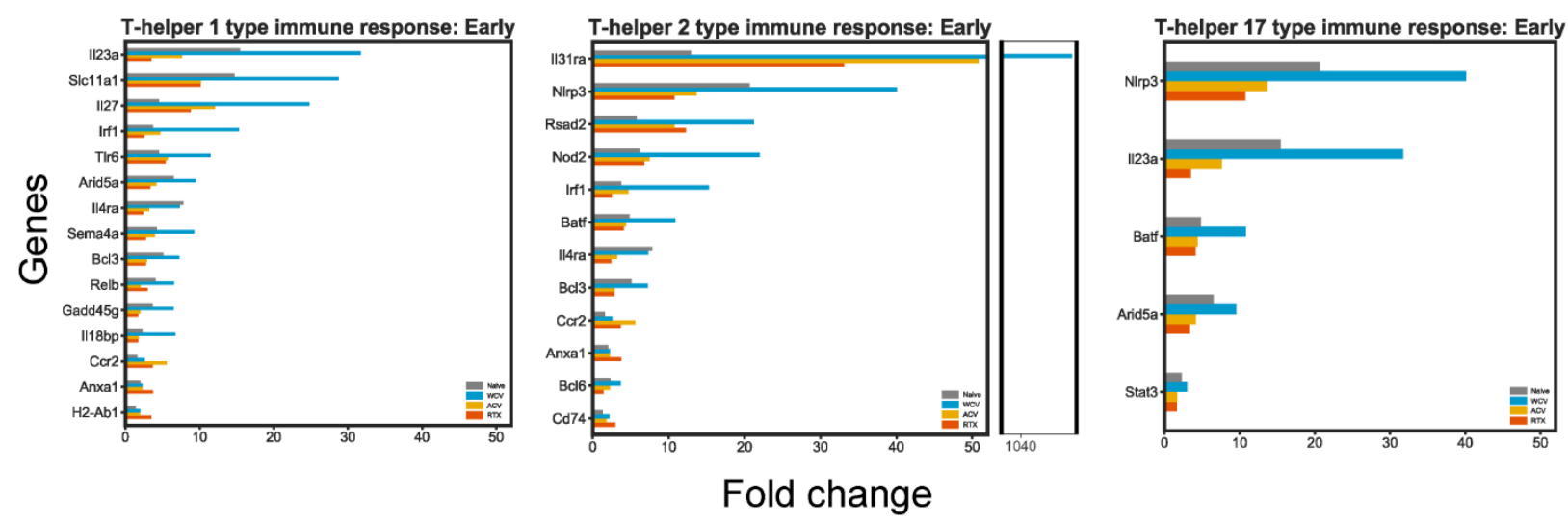

Supplementary Fig. 5: Gene expression profiles of $\mathbf{T}$ helper cell immune responses at early time point. Differentiated genes associated with (a) Th1, (b) Th2, and (c) Th17 immune responses. Fold changes are relative to NVNC mice. Genes represented are significantly different in at least one group, genes were sorted based on variance of fold change among the experimental groups. 
Supplementary Fig. 6: Repertoire of B cell clones identified from experimental groups at late time point.

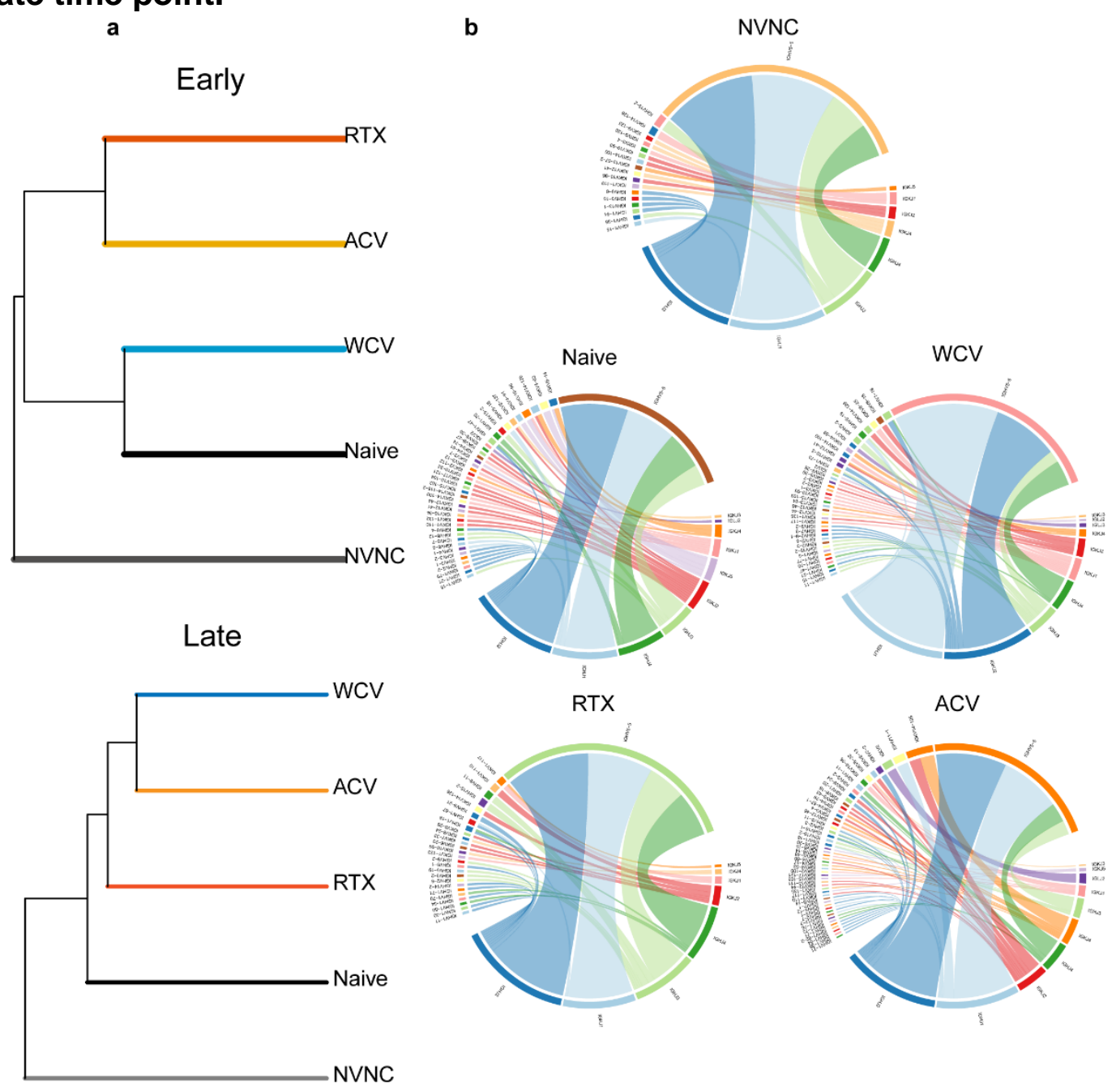

Supplemental Fig. 6: Repertoire of B cell clones identified from experimental groups at late time point. (a) Relativeness of immunoglobulin profiles between vaccinated and challenged, challenged, or non-challenged groups at late time point. (b) Immunoglobulin diversity and frequency of non-challenged control, experimental groups at late time points. Chord diagrams are used to visualize variable - joining segment diversity. Outer arcs demonstrate overall count of a particular variable (upper portion of chord diagram) or joining segment (lower portion of chord diagram) in each group. Inner ribbons represent the linked segments of a clonotype, while the thickness of the ribbon signifies frequency of the clonotype. 


\section{$\underline{\text { References }}$}

1 Paddock CD, Sanden GN, Cherry JD, Gal AA, Langston C, Tatti KM, et al. Pathology and Pathogenesis of Fatal Bordetella pertussis Infection in Infants. Clin Infect Dis 2008;47:328-38. https://doi.org/10.1086/589753.

2 Andreasen C, Powell DA, Carbonetti NH. Pertussis toxin stimulates IL-17 production in response to Bordetella pertussis infection in mice. PLoS One 2009;4:e7079. https://doi.org/10.1371/journal.pone.0007079.

3 Carbonetti NH. Pertussis toxin and adenylate cyclase toxin: Key virulence factors of Bordetella pertussis and cell biology tools. Future Microbiol 2010;5:455-69. https://doi.org/10.2217/fmb.09.133.

4 Carbonetti $\mathrm{NH}$. Contribution of pertussis toxin to the pathogenesis of pertussis disease. Pathog Dis 2015;73:ftv073. https://doi.org/10.1093/femspd/ftv073.

5 Carbonetti NH, Artamonova G V., Andreasen C, Bushar N. Pertussis toxin and adenylate cyclase toxin provide a one-two punch for establishment of Bordetella pertussis infection of the respiratory tract. Infect Immun 2005;73:2698-703. https://doi.org/10.1128/IAI.73.5.2698-2703.2005.

6 Sebo P, Osicka R, Masin J. Adenylate cyclase toxin-hemolysin relevance for pertussis vaccines. Expert Rev Vaccines 2014;13:1215-27. https://doi.org/10.1586/14760584.2014.944900.

7 Mattoo S, Cherry JD. Molecular pathogenesis, epidemiology, and clinical manifestations of respiratory infections due to Bordetella pertussis and other Bordetella subspecies. Clin Microbiol Rev 2005;18:326-82. https://doi.org/10.1128/CMR.18.2.326-382.2005.

8 Gold MS. Hypotonic-Hyporesponsive Episodes Following Pertussis Vaccination. Drug Saf 2002;25:85-90. https://doi.org/10.2165/00002018-200225020-00003.

9 Donnelly S, Loscher CE, Lynch MA, Mills KHG. Whole-cell but not acellular pertussis vaccines induce convulsive activity in mice: Evidence of a role for toxininduced interleukin-1?? in a new murine model for analysis of neuronal side effects of vaccination. Infect Immun 2001;69:4217-23. https://doi.org/10.1128/IAI.69.7.4217-4223.2001.

10 Ross PJ, Sutton CE, Higgins S, Allen AC, Walsh K, Misiak A, et al. Relative contribution of Th1 and Th17 cells in adaptive immunity to Bordetella pertussis : towards the rational design of an improved acellular pertussis vaccine. PLoS Pathog 2013;9:e1003264. https://doi.org/10.1371/journal.ppat.1003264.

11 Mills KHG, Ryan M, Ryan E, Mahon BP. A murine model in which protection correlates with pertussis vaccine efficacy in children reveals complementary roles for humoral and cell- mediated immunity in protection against Bordetella pertussis 
. Infect Immun 1998;66:594-602. https://doi.org/10.1371/journal.ppat.1003264.

12 Warfel JM, Merkel TJ. Bordetella pertussis infection induces a mucosal IL-17 response and long-lived Th17 and Th1 immune memory cells in nonhuman primates. Mucosal Immunol 2013;6:787-96. https://doi.org/10.1038/mi.2012.117.

13 Carbonetti NH. Pertussis leukocytosis: mechanisms, clinical relevance and treatment. Pathog Dis 2016;74:ftw087. https://doi.org/10.1093/femspd/ftw087.

14 Fedele G, Spensieri F, Palazzo R, Nasso M, Cheung GYC, Coote JG, et al. Bordetella pertussis commits human dendritic cells to promote a Th1/Th17 response through the activity of adenylate cyclase toxin and MAPK-pathways. PLoS One 2010;5:e8734. https://doi.org/10.1371/journal.pone.0008734.

15 Mills KHG, Ross PJ, Allen AC, Wilk MM. Do we need a new vaccine to control the re-emergence of pertussis? Trends Microbiol 2014;22:49-52. https://doi.org/10.1016/j.tim.2013.11.007.

16 Klein NP, Bartlett J, Fireman B, Baxter R. Waning Tdap Effectiveness in Adolescents. Pediatrics 2016;137:e20153326-e20153326. https://doi.org/10.1542/peds.2015-3326.

17 Warfel JM, Beren J, Merkel TJ. Airborne transmission of Bordetella pertussis . J Infect Dis 2012;206:902-6. https://doi.org/10.1093/infdis/jis443.

18 Althouse BM, Scarpino S V. Asymptomatic transmission and the resurgence of Bordetella pertussis . BMC Med 2015;13:146. https://doi.org/10.1186/s12916-0150382-8.

19 Betsou F, Sebo P, Guiso N. CyaC-mediated activation is important not only for toxic but also for protective activities of Bordetella pertussis adenylate cyclasehemolysin. Infect Immun 1993;61:3583-9.

20 Cheung GYC, Xing D, Prior S, Corbel MJ, Parton R, Coote JG. Effect of different forms of adenylate cyclase toxin of Bordetella pertussis on protection afforded by an acellular pertussis vaccine in a murine model. Infect Immun 2006;74:6797805. https://doi.org/10.1128/IAI.01104-06.

21 Guiso N, Szatanik M, Rocancourt M. Protective activity of Bordetella adenylate cyclase-hemolysin against bacterial colonization. Microb Pathog 1991;11:423-31. https://doi.org/10.1016/0882-4010(91)90038-C.

22 Wang X, Maynard JA. The Bordetella adenylate cyclase repeat-in-toxin (RTX) domain is immunodominant and elicits neutralizing antibodies. $\mathrm{J}$ Biol Chem 2015;290:3576-91. https://doi.org/10.1074/jbc.M114.585281.

23 Harvill ET, Cotter PA, Miller JF. Pregenomic comparative analysis between Bordetella bronchiseptica RB50 and Bordetella pertussis Tohama I in murine models of respiratory tract infection. Infect Immun 1999;67:6109-18.

24 Kirimanjeswara GS, Agosto LM, Kennett MJ, Bjornstad ON, Harvill ET. Pertussis toxin inhibits neutrophil recruitment to delay antibody-mediated clearance of 
Bordetella pertussis . J Clin Invest 2005;115:3594-601. https://doi.org/10.1172/JCl24609.

25 Andreasen $\mathrm{C}$, Carbonetti $\mathrm{NH}$. Role of neutrophils in response to Bordetella pertussis infection in mice. Infect Immun 2009;77:1182-8. https://doi.org/10.1128/IAI.01150-08.

26 Weiss AA, Mary GMS. Lethal infection by Bordetella pertussis mutants in the infant mouse model. Infect Immun 1989;57:3757-64.

27 Goodwin MS, Weiss AA. Adenylate cyclase toxin is critical for colonization and pertussis toxin is critical for lethal infection by Bordetella pertussis in infant mice. Infect Immun 1990;58:3445-7.

28 Locht C, Coutte L, Mielcarek N. The ins and outs of pertussis toxin n.d. https://doi.org/10.1111/j.1742-4658.2011.08237.x.

29 Froehlich. Beitrag zur pathologie des Keuchhustens. Jahrb Fur Kinderheilkd 1897;44:.

30 Meunier H. De la leucocytose dans la coqueluche. CR Soc Biol 1898;50:.

31 MORSE SI. STUDIES ON THE LYMPHOCYTOSIS INDUCED IN MICE BY BORDETELLA PERTUSSIS . J Exp Med 1965;121:49-68. https://doi.org/10.1084/jem.121.1.49.

32 Morse SI, Riester SK. Studies on the leukocytosis and lymphocytosis induced by Bordetella pertussis . I. Radioautographic analysis of the circulating cells in mice undergoing pertussis-induced hyperleukocytosis. J Exp Med 1967;125:401-8. https://doi.org/10.1084/jem.125.3.401.

33 Weiner ZP, Ernst SM, Boyer AE, Gallegos-Candela M, Barr JR, Glomski IJ. Circulating lethal toxin decreases the ability of neutrophils to respond to Bacillus anthracis. Cell Microbiol 2014;16:504-18. https://doi.org/10.1111/cmi.12232.

34 Higgs R, Higgins SC, Ross PJ, Mills KHG. Immunity to the respiratory pathogen Bordetella pertussis . Mucosal Immunol 2012;5:485-500. https://doi.org/10.1038/mi.2012.54.

35 Ross PJ, Sutton CE, Higgins S, Allen AC, Walsh K, Misiak A, et al. Relative contribution of Th1 and Th17 cells in adaptive immunity to Bordetella pertussis : towards the rational design of an improved acellular pertussis vaccine. PLoS Pathog 2013;9:e1003264. https://doi.org/10.1371/journal.ppat.1003264.

36 Alvarez Hayes J, Erben E, Lamberti Y, Principi G, Maschi F, Ayala M, et al. Bordetella pertussis iron regulated proteins as potential vaccine components. Vaccine 2013;31:3543-8. https://doi.org/10.1016/j.vaccine.2013.05.072.

37 Sawal M, Cohen M, Irazuzta JE, Kumar R, Kirton C, Brundler M-A, et al. Fulminant pertussis: A multi-center study with new insights into the clinicopathological mechanisms. Pediatr Pulmonol 2009;44:970-80. https://doi.org/10.1002/ppul.21082. 
38 Pierce C, Klein N, Peters M. Is leukocytosis a predictor of mortality in severe pertussis infection? Intensive Care Med 2000;26:1512-4.

39 Alves-Filho JC, Freitas A, Souto FO, Spiller F, Paula-Neto H, Silva JS, et al. Regulation of chemokine receptor by Toll-like receptor 2 is critical to neutrophil migration and resistance to polymicrobial sepsis. Proc Natl Acad Sci U S A 2009;106:4018-23. https://doi.org/10.1073/pnas.0900196106.

40 Raeven RHM, Van Der Maas L, Tilstra W, Uittenbogaard JP, Bindels THE, Kuipers B, et al. Immunoproteomic Profiling of Bordetella pertussis Outer Membrane Vesicle Vaccine Reveals Broad and Balanced Humoral Immunogenicity. J Proteome Res 2015;14:2929-42. https://doi.org/10.1021/acs.jproteome.5b00258.

41 Starost LJ, Karassek S, Sano Y, Kanda T, Kim KS, Dobrindt U, et al. Pertussis Toxin Exploits Host Cell Signaling Pathways Induced by Meningitis-Causing E. coli K1-RS218 and Enhances Adherence of Monocytic THP-1 Cells to Human Cerebral Endothelial Cells. Toxins (Basel) 2016;8:291. https://doi.org/10.3390/toxins8100291.

42 Cotoi OS, Dunér P, Ko N, Hedblad B, Nilsson J, Björkbacka H, et al. Plasma S100A8/A9 correlates with blood neutrophil counts, traditional risk factors, and cardiovascular disease in middle-aged healthy individuals. Arterioscler Thromb Vasc Biol 2014;34:202-10. https://doi.org/10.1161/ATVBAHA.113.302432.

43 Vogl T, Ludwig S, Goebeler M, Strey A, Thorey IS, Reichelt R, et al. MRP8 and MRP14 control microtubule reorganization during transendothelial migration of phagocytes. Blood 2004;104:4260-8. https://doi.org/10.1182/blood-2004-020446.

44 Kumar H, Kumagai Y, Tsuchida T, Koenig PA, Satoh T, Guo Z, et al. Involvement of the NLRP3 Inflammasome in Innate and Humoral Adaptive Immune Responses to Fungal -Glucan. J Immunol 2009;183:8061-7. https://doi.org/10.4049/jimmunol.0902477.

45 Gabrilovich DI. The Neutrophils: New Outlook for Old Cells. Singapore, UNITED STATES: Imperial College Press; 2014.

46 Mahon BP, Sheahan BJ, Griffin F, Murphy G, Mills KH. Atypical disease after Bordetella pertussis respiratory infection of mice with targeted disruptions of interferon-gamma receptor or immunoglobulin mu chain genes. J Exp Med 1997;186:1843-51.

47 Kirimanjeswara GS, Mann PB, Harvill ET. Role of antibodies in immunity to Bordetella infections. Infect Immun 2003;71:1719-24. https://doi.org/10.1128/iai.71.4.1719-1724.2003.

48 Bolotin DA, Poslavsky S, Mitrophanov I, Shugay M, Mamedov IZ, Putintseva E V, et al. MiXCR: software for comprehensive adaptive immunity profiling. Nat Methods 2015;12:380-1. https://doi.org/10.1038/nmeth.3364. 
$49 \mathrm{Xu} \mathrm{JL}$, Davis MM. Diversity in the CDR3 region of $\mathrm{V}(\mathrm{H})$ is sufficient for most antibody specificities. Immunity 2000;13:37-45. https://doi.org/10.1016/S10747613(00)00006-6.

50 Nguyen AW, Wagner EK, Laber JR, Goodfield LL, Smallridge WE, Harvill ET, et al. A cocktail of humanized anti-pertussis toxin antibodies limits disease in murine and baboon models of whooping cough. Sci Transl Med 2015;7:316ra195. https://doi.org/10.1126/scitrans/med.aad0966.

51 Im S-Y, Wiedmeier SE, Cho B-H, Lee DG, Beigi M, Daynes RA. Dual effects of pertussis toxin on murine neutrophils in vivo. Inflammation 1989;13:707-26. https://doi.org/10.1007/BF00914314.

52 Winter K, Zipprich J, Harriman K, Murray EL, Gornbein J, Hammer SJ, et al. Risk Factors Associated With Infant Deaths From Pertussis: A Case-Control Study. Clin Infect Dis 2015;61:1099-106. https://doi.org/10.1093/cid/civ472.

53 Heininger U, Klich K, Stehr K, Cherry JD. Clinical findings in Bordetella pertussis infections: results of a prospective multicenter surveillance study. Pediatrics 1997;100:E10. https://doi.org/10.1542/peds.100.6.e10.

54 Guiso N, Rocancourt M, Szatanik M, Alonso JM. Bordetella adenylate cyclase is a virulence associated factor and an immunoprotective antigen. Microb Pathog 1989;7:373-80. https://doi.org/10.1016/0882-4010(89)90040-5.

55 Torres CA, Iwasaki A, Barber BH, Robinson HL. Differential dependence on target site tissue for gene gun and intramuscular DNA immunizations. J Immunol 1997;158:4529-32.

56 Bassinet L, Fitting C, Housset B, Cavaillon JM, Guiso N. Bordetella pertussis adenylate cyclase-hemolysin induces interleukin- 6 secretion by human tracheal epithelial cells. Infect Immun 2004;72:5530-3. https://doi.org/10.1128/IAI.72.9.5530-5533.2004.

57 Raeven RHM, Brummelman J, Pennings JLA, Nijst OEM, Kuipers B, Blok LER, et al. Molecular signatures of the evolving immune response in mice following a Bordetella pertussis infection. PLOS One 2014;9:e104548. https://doi.org/10.1371/journal.pone.0104548.

58 Moreno G, Errea A, Van Maele L, Roberts R, Léger H, Sirard JC, et al. Toll-like receptor 4 orchestrates neutrophil recruitment into airways during the first hours of Bordetella pertussis infection. Microbes Infect 2013;15:708-18. https://doi.org/10.1016/j.micinf.2013.06.010.

59 Brickman TJ, Armstrong SK. The ornithine decarboxylase gene odc is required for alcaligin siderophore biosynthesis in Bordetella spp.: Putrescine is a precursor of alcaligin. J Bacteriol 1996;178:54-60. https://doi.org/10.1128/jb.178.1.54-60.1996.

60 Stainer DW, Scholte MJ. A Simple Chemically Defined Medium for the Production of Phase I Bordetella pertussis . J Gen Microbiol 1970;63:211-20. https://doi.org/10.1099/00221287-63-2-211. 
61 Evans MS, Chaurette JP, Adams ST, Reddy GR, Paley MA, Aronin N, et al. A synthetic luciferin improves bioluminescence imaging in live mice. Nat Methods 2014;11:393-5. https://doi.org/10.1038/nmeth.2839.

62 Damron FH, Oglesby-Sherrouse AG, Wilks A, Barbier M. Dual-seq transcriptomics reveals the battle for iron during Pseudomonas aeruginosa acute murine pneumonia. Sci Rep 2016;6:. https://doi.org/Artn 39172 10.1038/Srep39172.

63 Olivers JC. Venny. An interactive tool for comparing lists with Venn's diagrams n.d.

64 Carbon S, Ireland A, Mungall CJ, Shu S, Marshall B, Lewis S, et al. AmiGo: online access to ontology and annotation data. Bioinformatics 2009;25:288-9. https://doi.org/10.1093/bioinformatics/btn615.

65 Shugay M, Bagaev D V, Turchaninova MA, Bolotin DA, Britanova O V, Putintseva E V, et al. VDJtools: Unifying Post-analysis of T Cell Receptor Repertoires. PLoS Comput Biol 2015;11:e1004503. https://doi.org/10.1371/journal.pcbi.1004503. 


\section{Chapter 3 - Evaluation of adenylate cyclase toxoid antigen in acellular pertussis vaccines using a Bordetella pertussis challenge model in mice.}

Dylan T. Boehmª, Jesse M. Halla, Ting Y. Wong ${ }^{a}$, Andrea DiVenerec, Emel Sen-Kilic ${ }^{\mathrm{a}}$, Justin R. Beverea, Shelby D. Bradford ${ }^{a}$, Catherine B. Blackwood ${ }^{a}$, Cody Elkins ${ }^{a}$, Katherine A. DeRoos ${ }^{\mathrm{a}}$, Mary C. Gray ${ }^{\mathrm{b}}$, Garret Cooperd, Melinda E. Varneya, Jennifer A. Maynard $^{\mathrm{c}}$, Erik L. Hewlett ${ }^{\mathrm{b}}$, Mariette Barbiera, and F. Heath Damron ${ }^{\mathrm{a}}$

Department of Microbiology, Immunology, and Cell Biology, West Virginia University, Morgantown, WV, USA ${ }^{a}$; Department of Medicine, Division of Infectious Diseases and International Health, University of Virginia, Charlottesville, Virginia, USA ${ }^{\mathrm{b}}$; Department of Chemical Engineering, University of Texas at Austin, Austin, TX 78712, USA ${ }^{c}$; Department of Medicine, Section of Infectious Diseases, West Virginia University

Published in Infection and Immunity 2018:IAI.00857-17.

https://doi.org/10.1128/IAl.00857-17. 


\section{Abstract}

Bordetella pertussis (B. pertussis) is the primary causative agent of pertussis (whooping cough), which is a respiratory infection that leads to a violent cough and can be fatal in infants. There is a need to develop more effective vaccines because of the resurgence of cases of pertussis in the US since the switch from the whole cell pertussis vaccines (wP) to the acellular pertussis vaccines (aP; DTaP/Tdap). Adenylate Cyclase Toxin (ACT) is a major virulence factor of $B$. pertussis that is: 1) required for establishment of infection, 2) an effective immunogen, 3) a protective antigen. The Cterminal Repeats-In-Toxin domain (RTX) of ACT is sufficient to induce production of toxin neutralizing antibodies. In this study, we characterized the effectiveness of vaccines containing the RTX antigen against experimental murine infection with $B$. pertussis. RTX was not protective as a single-antigen vaccine against $B$. pertussis challenge, and adding RTX to $1 / 5^{\text {th }}$ human dose of aP vaccine did not enhance protection. Since the doses of aP used in murine studies are not proportionate to mouse/human body masses, we titrated the aP vaccine from $1 / 20^{\text {th }}$ to $1 / 160^{\text {th }}$ of the human dose. Mice receiving $1 / 80^{\text {th }}$ human aP dose had bacterial burden comparable to naïve controls. Adding RTX antigen to the $1 / 80^{\text {th }}$ aP base resulted in enhanced bacterial clearance. Inclusion of RTX induced: production of antibodies recognizing RTX, enhanced production of anti-PT, decreased secretion of pro-inflammatory cytokines such as IL-6, and decreased recruitment of total macrophages in the lung. This study shows that adding RTX antigen to an appropriate dose of aP vaccine can enhance protection against $B$. pertussis challenge in mice. 


\section{Introduction}

Whooping cough is a disease caused by an upper respiratory tract infection that is primarily caused by the Gram-negative pathogen, Bordetella pertussis (B. pertussis) and several other Bordetellae. These infections, commonly referred to as pertussis, can be fatal in infants, but can also cause significant suffering in children, adolescents, and adults. Fortunately, two generations of vaccines have been developed and have greatly reduced the incidence of pertussis in the US and worldwide. Killed whole cell vaccines (wP) were first reported in $1933^{1}$. With the development of the Kendrick test in 1947, wPs were standardized leading to an increase in vaccine efficacy such $80 \%$ of those immunized developed long-term immunity to pertussis ${ }^{2}$. Use of the wP resulted in drastic reduction of the incidence of whooping cough in the second half of the twentieth century. However, in the 1990s, due to reactogenicity and public concern, wPs were replaced with acellular vaccines (aP; DTaP; Tdap) containing 3-5 pertussis protein antigens and alum adjuvant ${ }^{3}$. Since the introduction of aPs, increases in pertussis cases throughout the US and Europe have been observed and a number of reasons have been postulated to explain their lack of complete efficacy of the aPs ${ }^{4}$.

One of the major issues with the aPs is that their efficacy wanes each year after administration 5,6. Recent data from the non-human primate model of pertussis demonstrates that while the aPs prevent the paroxysmal whooping cough, they do not protect from colonization and transmission ${ }^{7}$. In light of the increased incidence and our appreciation of the lack of efficacy of the aP, there is a need to develop another generation of vaccines with improved efficacy and duration of protection. Various approaches have been considered for the development of the new generation of pertussis vaccines, including: 1 ) addition of new antigens to the current aPs, 2) repolarization of the host Th-2 response triggered by alum-adjuvanted aPs to a Th-1/Th17 response by changing the adjuvant and adding new antigens, or 3) re-introduction of wPs (live attenuated or killed) either as primary immunization or as a re-polarizing booster. 
B. pertussis expresses pertussis toxin (PT) and adenylate cyclase toxin (ACT) to facilitate infection and impair the host immune response in a variety of ways. Detoxified PT antigens are included in all commercial aPs. When the aP was developed, ACT was recognized as an essential virulence factor, but as of the late 1980s, ACT had not been extensively evaluated as protective antigen and was not available in purified form. It was therefore not included in aP formulations. The cya $A$ gene encodes $A C T$, a soluble toxin of 1,706 amino acids ${ }^{8}$, comprised of an adenylate cyclase (AC) domain and a Repeats in Toxin (RTX) domain ${ }^{9}$. The RTX domain is responsible for the hemolytic activity of ACT ${ }^{10}$. The cyclase domain can be delivered to eukaryotic cells where it catalyzes ATP into supraphysiological concentrations of cAMP, which impairs many cell functions and leads to cell death ${ }^{9}$. B. pertussis requires ACT to establish lethal infection in neonatal mice ${ }^{11}$, where the toxin targets macrophages, neutrophils, dendritic cells, and other integrin $\alpha_{M} \beta_{2}$ expressing cells ${ }^{9}$. ACT has both adenylate cyclase and hemolytic activities and its hemolytic activity is required for $B$. pertussis penetration into the lung parenchyma ${ }^{12}$. Underscoring its role in disease, $A C$ enzyme activity has been detected in airway samples from a non-human primate model of pertussis infection ${ }^{13}$, and humans 13, and convalescent humans have antibodies against ACT 14,15. Furthermore, adenylate cyclase activity can be detected in WPs ${ }^{16}$ and in our own mass spectral analysis we have confirmed the presence of ACT in WP vaccines (unpublished).

In light of the role of ACT in virulence and the fact that $B$. pertussis infection induces production of anti-ACT antibodies, ACT has been evaluated as a protective antigen in pre-clinical murine immunization/challenge models. Before aPs replaced wPs in the US and Europe, Guiso et al. demonstrated that a polypeptide consisting of the AC domain purified from $B$. pertussis was a protective antigen in mice ${ }^{17}$. The same group demonstrated that full length toxin purified from $B$. pertussis was protective (16). Subsequent studies showed that recombinant ACT was also a protective antigen 18,19 and an adjuvant ${ }^{20}$. While immunization with ACT results in toxin neutralizing antibodies, the presence of these antibodies do not correlate directly with protection. However, antibodies that recognize the C-terminal domain (RTX) are protective ${ }^{19}$. These studies 
were further supported by evidence that the hemolytic domain only antigen (RTX) was protective in mice against sub-lethal and lethal challenge with $B$. pertussis ${ }^{19}$. In all of the aforementioned studies, ACT antigens were evaluated as single antigen formulations without any of the aP antigens. Cheung et al. observed that inclusion of enzymatically inactive recombinant ACT enhanced protection of the $\mathrm{aP}{ }^{21}$, and that unlike previous single antigen studies, neither active nor enzymatically inactive ACT protected alone ${ }^{21}$. The study also showed that inclusion of ACT in the aP skewed the Thelper cell responses from a humoral Th2 response to a more cell-mediated Th1 response ${ }^{21}$.

Multiple studies have demonstrated the protective capacity of ACT ${ }^{8}$. In order to determine the appropriate form of ACT for use in a human vaccine, it is necessary to identify a protective toxoid form that would be readily purified for mass production. Genetic mutation of the ACT coding sequence results in an enzymatically inactive form/molecule that is protective in mice ${ }^{21}$. However, the use of a full length ACT would be problematic to for use in human vaccines due to: 1 ) the susceptibility of the toxin to proteolytic degradation 22 and 2) overexpression of ACT leads to formation of inclusion bodies in the E. coli cytoplasm ${ }^{23-25}$. A recent study confirmed that ACT is prone to aggregation and proteolysis ${ }^{9}$. Wang et al. characterized a recombinant RTX antigen that was 1) readily purified, 2) immunodominant, and 3) elicited neutralizing antibody production ${ }^{26}$. While these results are very promising, it is essential to determine the efficacy of recombinant RTX antigen in pre-clinical models of pertussis, both alone and in combination with aP. It is likely that a new aP vaccine would still include PT, FHA, and FIM antigens. Our objective was to investigate the RTX antigen with the standard aP antigens as a multivalent formulation. In this present study, inclusion of the RTX antigen into an aP formulation was evaluated with a murine immunization and challenge model. We observed that immunization with RTX alone was not protective and furthermore when either RTX or full length ACT was added to the $1 / 5^{\text {th }}$ human dose, no significant enhancement of protection occurred. The use of high amounts of the human aP in mice (such as $1 / 4^{\text {th }}, 1 / 5^{\text {th }}$, or $1 / 10^{\text {th }}$ human dose) relates back to doses intended for commercial vaccine lot validation. It is known that as little as $1 / 50^{\text {th }}$ human dose of 
$\mathrm{aP}$ is protective in mice ${ }^{27}$. We hypothesized that identification of the minimal protective and maximal non-protective aP doses would allow us to investigate enhancement of protection by inclusion of new antigens, thereby refining this evaluation platform. By performing a vaccine titration study using two-fold dilutions, we were able to identify $1 / 80^{\text {th }}$ human dose of aP as a minimal protective dose. When the RTX antigen was added into the $1 / 80^{\text {th }}$ human dose, improved bacterial clearance was observed. Inclusion of RTX antigen also enhanced antibody production to PT demonstrating the adjuvant capacity of RTX. The enhanced protection correlated with 1) decreased proinflammatory cytokine production, 2) decreased blood and lung neutrophils, and 3) decreased macrophages in the lung. This study begins to redefine murine immunization and $B$. pertussis challenge model for evaluation of pertussis vaccines and sheds light on the positive effects of including RTX as an antigen in the acellular vaccines.

\section{Materials and Methods}

\section{ACT and RTX antigen expression and purification}

ACT and RTX antigens were expressed and purified as previously described ${ }^{9}$. Following purification, antigens were stripped of endotoxin with Dextoxi-Gel ${ }^{\mathrm{TM}}$ Endotoxin Removing Gel (Thermo Scientific, 20399) and Zeba Spin columns (Thermo Scientific, 89896). Endotoxin purity was quantified with Pierce ${ }^{\circledR}$ LAL Chromogenic Endotoxin Quantitation Kit (Thermo Scientific, 88282).

\section{Composition of vaccines used in study and administration}

The INFANRIX (GSK) human vaccine (diphtheria, tetanus, and pertussis) was the aP used in this study. For the purpose of vaccinating mice, $1 / 5^{\text {th }}$ of the human dose was used $(100 \mu \mathrm{l})$. To test ACT as an additional antigen, full length ACT or RTX-ACT were purified as previously described ${ }^{9}$. Equimolar amounts of full length ACT (10 $\left.\mu \mathrm{g}\right)$ or RTX $(5.6 \mu \mathrm{g})$ were used for vaccination. Vaccine compositions are shown in Table S1. Alum and MPLA were obtained from InvivoGen. The National Institute for Biological Standards and Control WHO standard whole cell pertussis vaccine (NIBSC code 94/532) was used as the WP. One ampule of NIBSC WP contains 40 IU (10 human 
doses). For murine vaccination, the whole ampule was diluted in $1 \mathrm{ml}$ of sterile PBS and $20 \mu \mathrm{l}\left(1 / 5^{\text {th }}\right.$ of the human dose, $66 \mu \mathrm{g}$ of total protein) were used. The NIBSC wP standard used in this study has been used in other studies as a WP vaccine ${ }^{28}$. The NIBSC wP does not contain alum adjuvant, whereas commercial WP vaccine contain alum to induce antibody production to the Diptheria and Tetanus toxoids. The adjuvant alum was only added to single antigen vaccines (Table S1). INFANRIX is formulated with alum, thus we did not include additional alum for aP titration and aP supplementation with RTX. Unvaccinated mice received $200 \mu \mathrm{l}$ of sterile phosphate buffered saline (PBS) as vehicle control. Prior to administration, aP, aP-ACT, aP-RTX, and WP doses were all diluted into a final volume of $200 \mu \mathrm{l}$ with sterile PBS and administered by intraperitoneal injection.

\section{B. pertussis strains and growth conditions}

B. pertussis strain UT25 (UT25Sm1) ${ }^{29}$ was cultured on Bordet Gengou (BG) agar (Remel) ${ }^{30}$ supplemented with 15\% de-fibrinated sheep blood (Hemostat Laboratories) for $48 \mathrm{~h}$ at $36{ }^{\circ} \mathrm{C}$. Bacteria were transferred from BG plates to a flask with $20 \mathrm{ml}$ of modified Stainer-Scholte liquid medium (SSM) ${ }^{31}$. SSM cultures were not supplemented with cyclodextrin (heptakis). B. pertussis cultures in SSM were grown for $\sim 22 \mathrm{~h}$ at $36^{\circ} \mathrm{C}$ with shaking at $180 \mathrm{rpm}$ at which time the $\mathrm{OD}_{600}$ was $\sim 0.6$.

\section{Vaccination and challenge with $B$. pertussis}

Four week old CD-1 (outbred; strain code 022) mice were obtained from Charles River. Mice were vaccinated at 5 weeks of age with the vaccines described (Table S1), and then boosted with the same vaccines 21 days later. Thirty-eight days post initial vaccination, $B$. pertussis UT25 was grown as described above, and cultures were diluted to provide a challenge dose of $2 \times 10^{7}$ colony forming units (CFU) in $20 \mu$. Mice were anesthetized by intraperitoneal injection of $200 \mu \mathrm{l}$ of ketamine $(6.7 \mathrm{mg} / \mathrm{ml})$ and xylazine $(1.3 \mathrm{mg} / \mathrm{ml})$ in $0.9 \%$ saline. Mice received $20 \mu \mathrm{l}$ total challenge dose by pipetting $10 \mu \mathrm{l}$ directly into each nostril of the mouse. Four to eight mice per group were challenged, and at 1, 3, and 8 days post infection, mice were euthanized. Blood was 
collected by cardiac puncture and serum was separated by centrifugation through a BD Microtainer blood collector and stored at $-80^{\circ} \mathrm{C}$ until analysis. Trachea and lungs were removed and separately homogenized with Dounce homogenizers for determination of bacterial burden in each tissue. To determine the number of $B$. pertussis in the nares, 1 $\mathrm{ml}$ of PBS was flushed through the nares and collected. Serial dilutions in PBS were plated on BG containing streptomycin $(100 \mu \mathrm{g} / \mathrm{ml})$ to ensure that only UT25 B. pertussis were cultured. All murine infection experiments were performed according to protocols approved by the West Virginia University Animal Care and Use Committee (protocol number 14-1211.10).

\section{Cytokine analysis}

Lung homogenates were pelleted by centrifugation and the supernatants were collected and stored at $-80^{\circ} \mathrm{C}$ until analysis. The concentration of cytokines in the lungs and serum of vaccinated and challenged mice was determined by Raybiotech Mouse Inflammation Array C1 (AAM-INF-1-8) following the manufacturer's instructions. A total of $100 \mu$ l of lung homogenate supernatants or serum were pooled from 4 mice for each analysis. Quantitative analysis of the concentration of cytokines in the lungs mice was determined Meso Scale Discovery V-PLEX Proinflammatory Panel (K15048G-1) or Mouse IL-17 Ultra-Sensitive kits (K152ATC-1) per the manufacturer's instructions.

\section{Adenylate cyclase toxin neutralization assay}

ACT is, through a combination of mechanisms, cytotoxic for J774A.1 (ATCC $®$ TIB-67 TM) macrophage-like cells and protection against this effect is the basis for the toxin neutralization assay ${ }^{32}$. J774A.1 cells were cultured in Dulbecco's modified Eagle's medium with $25 \mathrm{mM}$ glucose (Gibco). J774A.1 cells $(30,000$ in $90 \mu \mathrm{l})$ were seeded in each well of a 96-well plate and allowed to attach overnight. ACT was incubated with serum samples for 10 min with mixing at $4^{\circ} \mathrm{C}$, and added to the cells at a final ACT concentration of $80 \mathrm{ng} / \mathrm{ml}$. Cells were then incubated at $37^{\circ} \mathrm{C}$ for $2 \mathrm{~h}$. The number of viable cells was determined using the CCK8 assay (Dojindo Laboratories), which measures reduction of WST-8, a water-soluble tetrazolium salt, by dehydrogenases in 
viable cells. Percent viable cells was determined for ACT alone and for serum + ACT compared to control cells by the following equation [(treatment - media without cells) / (control - media without cells)] $\times 100$.

\section{Preparation of flow cytometry samples and analysis}

Blood, lung, and cells isolated from nasal lavage or lung were analyzed by flow cytometry. A $100 \mu \mathrm{l}$ sample was removed from lung homogenate of all samples and filtered through a $70 \mu \mathrm{m}$ cell strainer, then centrifuged at $1,000 \times g$ for 5 min to pellet cells. Supernatant was removed, Pharmlyse red blood cell (RBC) lysis buffer (BD Biosciences) was added, and samples were incubated at $37^{\circ} \mathrm{C}$ for 2 min before being pelleted by centrifugation at $1,000 \times g$ for 5 min. Cells were finally re-suspended in PBS + 1\% Fetal Bovine Serum (FBS). To prepare blood samples for flow cytometry, blood collected by cardiac puncture was placed in ethylenediaminetetraacetic acid (EDTA) containing tube (BD Biosciences) and RBC were lysed using Pharmlyse with a 15 min room temperature incubation. Cells were then pelleted by centrifugation at 1,000 $\mathrm{g} g$ for 5 min and re-suspended in PBS $+1 \%$ FBS. Cells isolated by nasal lavage are in a single cell suspension by nature, thus only required centrifugation at 1,000 $\mathrm{x} g$ for 5 min to concentrate samples. All cell suspension samples were incubated on ice in PBS and 1\% FBS for blocking for $15 \mathrm{~min}$. Antibodies against specific cell surface markers were added to cell suspensions and incubated in the dark for $1 \mathrm{~h}$ at $4^{\circ} \mathrm{C}$ : PE-conjugated GR-1 (BD, 553128), Alexa Fluor 700-conjugated CD-11b (Biolegend, 101222), PerCP/Cy5.5conjugated F4/80 (Biolegend, 123128). Lung, blood, and nasal lavage suspensions were pelleted and resuspended in PBS prior to analysis. Samples were read using an LSR Fortessa (BD) flow cytometer, and analyzed using the software FlowJo v10 (FlowJo, LLC). Neutrophils and macrophages were classified as CD-11 $\mathrm{b}^{+} \mathrm{Gr}-\mathrm{1}^{+}$single, live cells or $C D-11 b^{+} F 4 / 80^{+}$single, live cells, respectively.

\section{Serological analysis of $B$. pertussis specific antibodies}

Vaccinated and challenged mouse serological responses to RTX ${ }^{26}$, PT (LIST

Biologicals), filamentous hemagglutinin (FHA; Enzo Life Sciences), and 
lipooligosaccharide (LOS; LIST Biologicals) were determined by enzyme-linked immunosorbent assays (ELISA). High-binding 96-well ELISA plates were coated overnight at $4^{\circ} \mathrm{C}$ with $50 \mu \mathrm{l}$ of purified RTX, PT, FHA in PBS at a concentration of 1 $\mu \mathrm{g} / \mathrm{mL}$ or LOS at $5 \mu \mathrm{g} / \mathrm{ml}$. IgG2a monoclonal m1B7 ${ }^{33}$ (anti-PT) and IgG2a monoclonal M1H5 ${ }^{26}$ (anti-RTX) were obtained from Dr. Jennifer Maynard. To measure FHA or LOS responses, polyclonal murine anti-FHA (Cat \#JNIH-11, NIBSC) or murine IgG3 monoclonal antibody D26E (Thermo Scientific) were used, respectively. Plates were washed with PBS + 1\% Tween 20 (PBS-T), then blocked with 5\% milk in PBS-T for $2 \mathrm{hr}$ at $37^{\circ} \mathrm{C}$. Sera were diluted to a concentration in the linear detection range for each antigen. Plates were incubated for $2 \mathrm{hr}$ at $37^{\circ} \mathrm{C}$ with serially diluted serum samples from vaccinated groups. Following 3 PBS-T washes, goat anti-mouse IgG secondary antibody conjugated to alkaline phosphatase (AP) (Southern Biotech) was diluted at 1:2,000 in blocking buffer and added to each well, and incubated $1 \mathrm{~h}$ at $37^{\circ} \mathrm{C}$. Antibody standards were prepared from anti-PT, anti-FHA, anti-LOS, anti-RTX by 2 -fold dilutions from $100 \mu \mathrm{g} / \mathrm{mL}$. Plates were washed, then developed for 30 min with $100 \mu \mathrm{l} p$ nitrophenyl phosphate. Colorimetric signal was determined by measuring sample absorbance at 450nm using Spectramax i3 (Molecular Devices). Serum antibody concentration was calculated by plotting absorbance values of samples to absorbance of known concentrations of control antibodies. Values were plotted using a logarithmic scale, and fit to a four-parameter equation. Relative EC50 was determined by fitting the absorbance values of half of the maximal response to the four-parameter equation. Dilution of EC50 was determined by plotting the calculated antibody sample concentrations to sample dilution factors and fit to a four-parameter logistic curve ${ }^{34}$. Dilutions were accounted for by multiplying antibody concentration by dilution factor.

\section{Statistical Analysis}

Experiments in this study were performed with a minimum of four biological replicates. Data were statistically analyzed with GraphPad Prizm 7. Comparisons between single groups were performed using unpaired two-tailed $t$-tests and comparisons between multiple groups were performed using one-way ANOVA with a Tukey test for multiple comparisons. 


\section{Heat map modeling and visualization of the effects of immunization on correlates of protection}

A master file containing all of the data collected during this project was compiled and linear regressions were performed for each variable measured in response to vaccination and challenge (Matlab, Mathworks) to identify variables that correlate with overall protection against $B$. pertussis challenge. A subset of those variables were then extracted and used to plot visual representations of the overall correlates of protection. For each variable, modulations were calculated as the difference between the averaged responses of the non-challenged / non-vaccinated mice, and the averaged response of the mice in each experimental group. To establish a colorimetric scale, green was set as equal to the non-challenged / non-vaccinated mice, red was used for the maximal relative increase for that variable, and blue was used for the minimal relative value for that variable.

\section{Results}

Immunization with RTX alone does not enhance clearance of B. pertussis in CD-1 mice.

ACT plays a key role in the pathogenesis of $B$. pertussis and many studies have shown that both native and recombinant ACTs can be adjuvants and/or antigens for protection against pertussis in murine challenge studies ${ }^{17-20,35-39}$. In this study, we aimed to systematically evaluate the RTX antigen (toxoid version of ACT) for inclusion into the acellular pertussis vaccine. Wang et al. previously described that recombinant RTX antigen was immunogenic and readily purified, making it an attractive candidate for further evaluation. In our previous study using NECre luc mice (transgenic strain specifically used for tracking neutrophils in live mice ${ }^{40}$ ), we did not observe protection when mice were immunized with RTX + alum adjuvant but we did observe the presence of antibodies that recognize RTX antigens via ELISA (manuscript in review). Here, we immunized outbred CD-1 mice with the RTX antigen (Table S1) formulated with alum, which is a Th2 adjuvant, or monophosphoryl lipid A, (MPLA) which is a TLR4 agonist 
that induces Th1 responses. CD-1 mice were immunized intraperitoneally with PBS vehicle control (naïve) or $1 / 5^{\text {th }}$ human dose of aP as a positive control for protection. Twenty one days later the mice were boosted with the same vaccines. Two weeks after boost, each of these groups of mice were challenged with $2 \times 10^{7} \mathrm{CFU}$ of $B$. pertussis strain UT25 by intranasal administration. When this challenge dose of $B$. pertussis is intranasally administered to anesthetized mice, $26 \%$ of the initial dose is recoverable from the lung at $30 \mathrm{~min}$ post challenge and only $5 \%\left(10^{6} \mathrm{CFU}\right)$ is recoverable at $4 \mathrm{~h}$ post challenge (data not shown). These data suggest that many of the $B$. pertussis that were instilled by intranasal administration are killed by innate immune responses during the early stages of infection. However, this dose is sufficient to establish respiratory infection, as $4 \times 10^{6} \mathrm{CFU}$ can be recovered in the airways $24 \mathrm{hr}$ post challenge in naïve (PBS control immunized) CD-1 mice (Fig. 1A).

At one and three days post $B$. pertussis challenge, we enumerated the viable bacteria in the lungs, nares and trachea of naïve, aP, RTX+alum, and RTX+MPLA immunized CD1 mice (Fig. 1A and Fig. S1). The aP immunized mice had $99.75 \%$ less $B$. pertussis at day 1 post challenge than naïve mice (Fig. $1 \mathrm{~A}$ ), and by day 3 , the bacterial load was at or below our detection limit of 100 CFUs. However, mice immunized with RTX+alum or RTX+MPLA, were not significantly protected as they contained the same amount of viable $B$. pertussis as naïve mice (Fig. $1 A$ and Fig. S1AB). Interestingly, ELISAs were performed to measure anti-ACT antibodies and showed that mice vaccinated with RTX in combination with alum or MPLA induced detectable levels of antibodies in sera and lung homogenates (data not shown).

Vaccination with wP, aP, aP+RTX, and or aP+ACT protect against $B$. pertussis challenge. We hypothesized that inclusion of RTX into a standard acellular formulation would enhance protection against $B$. pertussis challenge in mice. To test this hypothesis, we compared aP and wP to aP supplemented with ACT or RTX in a murine vaccination challenge study. CD-1 mice were immunized as indicated in Table S1 and boosted three weeks post initial vaccination. At day 38 post initial vaccination, the mice were challenged with the $B$. pertussis strain UT25. At 1,3 , and 8 days post challenge, mice were euthanized for determination of bacterial burden and collection of tissues and 
samples for analysis. Naïve mice harbored similar amounts of $B$. pertussis in the lungs (Fig.1B), trachea and nares (Fig. S1CD) as those observed previously (Fig. 1A). wP and aP-vaccinated mice had significantly lower bacterial loads in the airways than nonvaccinated mice, and aP mice had the lowest bacterial burden at day 1 and 3 . By day 8 , all wP or aP immunized mice had cleared the infection to our detection limit. (Fig. 1B). Overall these data show that in CD-1 mice with UT25 as challenge strain, the $1 / 5^{\text {th }}$ human dose of aP is protective and that at that dose, adding RTX or ACT does not enhance bacterial clearance.

\section{Serological analysis of naïve, wP, aP, $\mathrm{AP}+\mathrm{ACT}$, and $\mathrm{aP}+\mathrm{RTX}$ immunized mice.} ELISAs were performed and we observed that alum and MPLA formulated RTX or ACT vaccines, induced antibodies that recognize ACT (data not shown). Additionally, $1 / 5^{\text {th }}$ aP supplemented with either RTX or ACT also induced detectable antibody production. Surprisingly, we did not observe any antibodies that would recognize ACT in the WP immunized mice (data not shown). The lack of anti-ACT in WP immunized mice is likely due to the fact that when we analyze the WP with shotgun proteomics, we only observe 4 peptides of ACT per 1,000 total peptides in the whole cell vaccine, which shows ACT makes up a minor amount of the total protein content (data not shown).

\section{AC toxin neutralizing capacity of serum antibodies.}

Due to the lack of noticeable effects on the rate of clearance of $B$. pertussis, it was unclear that those antibodies influenced clearance of $B$. pertussis in the mouse. ACT has potent toxic effects against CD-11b/CD-18-expressing phagocytes, such as macrophages or neutrophils ${ }^{41-43}$. To determine the functional capacity of the serum antibodies induced via immunization, we utilized the AC toxin neutralization assay with J774A.1 cells ${ }^{32}$. Only sera obtained from mice vaccinated with $\mathrm{aP}+\mathrm{ACT}$ or $\mathrm{aP}+\mathrm{RTX}$ were able to neutralize the toxicity of ACT in J774A.1 macrophages (Fig. 1C). We did not observe detectable anti-ACT antibodies in the serum from wP mice, and this serum did not neutralize ACT in vitro (Fig. 1C). The AC toxin neutralization data suggest that immunization with RTX can induce production of antibodies that can neutralize the AC toxin in vitro. 
Identification of the optimal aP murine dose to determine synergy between aP and RTX. Our data and previous published studies ${ }^{9}$ show that RTX is immunogenic and could contribute to protection against $B$. pertussis when used as an antigen. However, we hypothesized that the dose of aP used (1/5 human) in these studies is too high to observe synergy between the aP and RTX. Therefore, we sought to define a physiologically appropriate aP dose, for the mouse, that could be used to evaluate the effects of inclusion of new antigens into the aP. Guiso et al. demonstrated that 1 full human dose of DTaP was most protective (SmithKline Beecham Biologicals) ${ }^{44}$ and that $1 / 4^{\text {th }}$ and $1 / 10^{\text {th }}$ of the human dose were protective yet required more time to result in sterilizing immunity compared to a full human dose. Alvarez et al. ${ }^{45}$ used $1 / 5^{\text {th }}$ human dose and supplemented with an iron regulated protein, AfuA (BP1605) and observed modest improvement in bacteria clearance compared to $1 / 5^{\text {th }}$ human $D T a P$ (INFANRIX®; GSK). In other recent studies, $1 / 50^{\text {th }}$ human human DTaP (INFANRIX®; GSK) was partially protective in BALB/c mice challenged with B3 lineage strain B1917 27,46 . These two studies encouraged us to determine if lowering the aP dose could allow us to detect measurable improvements in bacterial clearance when additional antigens improve the vaccine.

CD-1 mice were immunized mice with aP doses of $1 / 20,1 / 40,1 / 80$, and $1 / 160^{\text {th }}$ human dose (Table S2) to titrate the vaccine and identify both the effective or non-effective doses. These mice were challenged with $B$. pertussis, and viable bacteria in the lungs, trachea, and nasal lavage were determined at 1 and 3 days post challenge (Fig. 2). At day 1 post challenge, the $1 / 20^{\text {th }}$ aP group had the least bacterial burden and at day 3 , both the $1 / 20^{\text {th }}$ and $1 / 40^{\text {th }}$ were near our detection limit (Fig. $2 A B$ ) in the lungs. A similar trend was observed in the trachea and nasal washes (Fig. 2CDEF). However, the $1 / 80^{\text {th }}$ aP group had $3.5 \log$ more viable $B$. pertussis in the lungs than the $1 / 40^{\text {th }}$ aP group, suggesting $1 / 80^{\text {th }}$ was below the aP dose for maximum protection in CD-1 mice (Fig. 2B). The non-effective dose for trachea seemed to be higher than $1 / 160^{\text {th }}$ (Fig. 2CD), whereas the non-effective dose for the nasal lavage was $1 / 160^{\text {th }}$ (Fig. 2EF). Based on these data, we selected the $1 / 40^{\text {th }}$ (minimal effective dose) and the $1 / 80^{\text {th }}$ (maximal noneffective dose) to supplement with AC toxoid antigen (RTX). 
We hypothesized that neutralization of ACT in vivo would enhance bacterial clearance and that inclusion of RTX would improve the protection provided by the $1 / 80^{\text {th }}$ dose as demonstrated by decreasing viable bacteria in the lung. Testing the inclusion of RTX in the $1 / 40^{\text {th }}$ dose would allow for evaluation of other subtler variables such as cytokines production, phagocyte recruitment, and serology. CD-1 mice were therefore immunized with either $1 / 40^{\text {th }}$ or $1 / 80^{\text {th }}$ dose aP supplemented with RTX antigen (Table S2). Inclusion of RTX into the $1 / 40^{\text {th }}$ aP did not affect the bacterial burden at day 3 in the lungs (Fig. 2B) compared to $1 / 40^{\text {th }}$ aP alone, as we predicted. When RTX was added to $1 / 80^{\text {th }} \mathrm{aP}$, we saw a significant decrease in viable bacteria in the lungs at day 3 (Fig. 2B). In light of this observation, it was necessary to measure other metrics in addition to viable bacteria to further support this finding.

In the trachea and nasal lavage, we observed the similar inverse relationship between bacterial burden and decreasing vaccine concentration as in the lungs. In the trachea, there was minimal improvement of bacterial clearance with inclusion of RTX to the $1 / 40^{\text {th }}$ or $1 / 80^{\text {th }}$ base $\mathrm{aP}$. In the nares, adding RTX to $1 / 40^{\text {th }}$ aP decreased the bacterial burden at day 1 but this difference was not significant $(p=0.29)$, and no differences were observed at day 3. Overall, these data highlight the need to consider the location

of $B$. pertussis in the host in respect to vaccine efficacy in a challenge trial and also the utility of identifying sub-effective murine vaccine doses to determine if there are effects of adding new antigens into a formulation.

\section{Serological analysis of antibodies recognizing PT and RTX antigens.}

In our aP immunization titration strategy, we expected that as the aP dose decreased, the concentration of anti-PT in the serum of immunized and challenged mice would also decrease. Given that mice immunized with $1 / 40^{\text {th }}$ aP had minimal bacterial burden in all respiratory tract locations (Fig. 2), we hypothesized that $1 / 40^{\text {th }}$ aP would induce production of high concentrations of anti-PT antibodies in serum. We also hypothesized that inclusion of the RTX antigen into the $1 / 40^{\text {th }}$ dose would result in production of significant amount of anti-RTX antibodies. As expected, immunization with RTX in both $1 / 40^{\text {th }}$ and $1 / 80^{\text {th }}$ aP doses induced production of antibodies that recognize the RTX 
antigen (Fig. 3AB). Surprisingly, there was very little difference between the amounts of detectable anti-RTX in $1 / 40^{\text {th }}$ and $1 / 80^{\text {th }}$ despite the fact that there was $50 \%$ less alum adjuvant in $1 / 80^{\text {th }}$ compared to $1 / 40^{\text {th }}$. We also observed that as we decreased PT antigen and alum adjuvant by diluting the vaccine, less anti-PT was produced in response to $B$. pertussis challenge (Fig.3CD). At day 1 we saw a dose response curve for anti-PT respective of the aP dose (Fig. $3 C$ ). Anti-PT decreased as would be expected since PT antigen and alum adjuvant are decreasing respective to the vaccine dose.

The addition of RTX into the $1 / 40^{\text {th }}$ and $1 / 80^{\text {th }}$ doses significantly increased antibody production to PT compared to same dilution of aP alone $(p=0.045$ and $p=0.0039$, respectively) at day 1 post challenge. Yet these levels of anti-PT drastically decreased at day 3 post challenge (Fig. 3D). This was a puzzling observation. There are several possibilities to explain this phenomenon. These ELISAs are from serum samples and it is possible that the high amounts of anti-PT have left the serum and are in the lung or upper respiratory tract closer to where the pathogens are infecting. The second possibility is that neutralization of ACT in vivo could decrease antibody production, which is less required due to bacterial clearance. More kinetics studies should be performed on these dual antigen considerations, especially with antigens like PT and RTX which both are classified as immunogens due to their adjuvant properties.

\section{Analysis of cytokine secretion as a function of aP immunization and inclusion of RTX antigen.}

Due to the fact that including RTX into the $1 / 80^{\text {th }}$ aP dose helps lower the bacterial burden in the lungs, we then characterized lung cytokine profiles to better understand the type of immune signaling factors released during infection and their association with bacterial clearance. To do so, we measured cytokines in lung homogenate of vaccinated and naïve mice at 1 and 3 days post-challenge. IL-6 is one of the most important cytokines involved in the immune response to $B$. pertussis. Respiratory epithelial cells and macrophages have increased IL-6 mRNA and protein in response to $B$. pertussis in vitro ${ }^{47,48}$ and C57BL/6 mice have high IL-6 secretion in response to $B$. 
pertussis infection ${ }^{48}$. Specifically, ACT has been shown to induce IL-6 expression ${ }^{49}$. Furthermore, IL-6 knockout mice have increased $B$. pertussis burden in the respiratory tract compared to wild type mice due to decreased recruitment of leukocytes ${ }^{48}$. Based on these studies and our preliminary cytokine data (Fig. S2), we anticipated that as we titrated the aP dose down, we would observe increased IL-6 correlating with the increased bacterial burden (Fig. 4AB). If antibodies against RTX can neutralize ACT in vivo, we expected to observe decreased IL-6 levels in RTX vaccinated mice. Our data confirmed this idea at day 1 , but at day 3 post challenge, RTX did not affect IL-6 secretion in the $1 / 80^{\text {th }}$ vaccine dilution group (Fig. 4B).

IL-6 is related to induction of Th17 responses, and in the case of $B$. pertussis infection, PT facilitates induction of IL-17 production typically around 7 days post $B$. pertussis challenge ${ }^{50}$. At day 1 , there were no statistically significant changes were observed in IL-17 across the groups. However, at day 3, as the aP dose decreased, IL-17 was slightly increased until it was significantly different at $1 / 160^{\text {th }}$ human dose (Fig. 4D). It was surprising to observe that naïve infected mice did not have significantly more IL-17 than mice vaccinated with the $1 / 160^{\text {th }}$ dose. We interpret these data to mean that: 1) 1 and 3 days are early for IL-17 responses to $B$. pertussis as we saw biological variability within the groups and 2) these diluted vaccines are likely capable of eliciting PTneutralizing antibodies. We did not observe any effect on IL-17 concentrations due to including RTX into either the $1 / 40^{\text {th }}$ or $1 / 80^{\text {th }}$ (Fig. $4 C D$ ).

B. pertussis challenge of mice induces interferon gamma (IFN- $\gamma$ ) expression, which is necessary for clearance of $B$. pertussis from mice ${ }^{51}$. Furthermore, IFN- $\gamma$ receptor deficient mice challenged with $B$. pertussis display atypical disease leading to dissemination of $B$. pertussis and death of the mice within several weeks ${ }^{52}$. Since aP immunization results in Th2 polarization, we expected that as the aP vaccine was titrated to less effective doses, we would see an increase in IFN- $\gamma$ induced by the natural Th1 response to $B$. pertussis infection ${ }^{53}$. At day 1 , the $1 / 40^{\text {th }}$ aP and the $1 / 80^{\text {th }}$ aP displayed similar IFN-y levels in the lung. However, when RTX was added to the $1 / 80^{\text {th }}$ we observed a striking reduction of IFN- $\gamma$ at day 1 only. It is also interesting to note that naïve infected mice had lower IFN-y than $1 / 40^{\text {th }}, 1 / 80^{\text {th }}$, or $1 / 160^{\text {th }}$ aP groups at 
day 1 (Fig. 4E), yet high IFN-y was observed in the naïve infected mice at day 3 (Fig. $4 \mathrm{~F})$.

IL-1 $\beta$ mediates clearance of $B$. pertussis and resolution of inflammation in mice ${ }^{54}$. IL-1 $\beta$ deficient mice have high amounts of IL-17 that are observed at 7 days post challenge and high IFN-y at 14 days post challenge ${ }^{54}$. We wondered if neutralization of ACT in vivo would alter IL-1 $\beta$ production in the lungs of the mice. All challenged mice had very similar IL-1 $\beta$ levels at day 1 (Fig. $4 G$ ) and the levels of IL-1 $\beta$ were lowest in the $1 / 20^{\text {th }}$ aP group at day 3. Addition of RTX did not significantly decrease IL-1 $\beta$ which suggests IL-1 $\beta$ may be induced independently of ACT activity or bacterial burden.

We measured other cytokines such as IL-5, TNF- $\alpha$, KC Gro, IL-12p70, IL-10, IL-2, and IL-4, in the lung homogenates of the vaccine groups (Fig. S3 and S4). Vaccination with aP+RTX resulted in lower IL-5 at day 1 in both $1 / 40^{\text {th }}$ and $1 / 80^{\text {th }}$ groups, yet these groups returned to base aP IL-5 levels at day 5 (Fig. S4). IL-2 followed a similar trend as IL-5 (Fig. S4). We observed that Th1 related responses such as IFN- $\gamma$ were decreased by the addition of RTX to the aP.

\section{Neutrophil infiltration kinetics are not affected by inclusion of RTX into the aP.}

Pertussis is characterized by an increase in the number of circulating white blood cells. This phenomenon known as leukocytosis can lead to lung hypertension and death in the most severe cases. ACT targets the CR3 receptor expressed on many cell types including neutrophils ${ }^{55}$. Based on this, we hypothesize that neutralization of $A C T$ in vivo would alter neutrophil kinetics and recruitment profiles in the blood and airway. Andreasen and Carbonetti reported that in naïve mice, neutrophils did not play a role in

clearance of $B$. pertussis from the airway ${ }^{50,56}$. On the other hand, neutrophils played an essential role in immune mice in the same study. Another study showed: 1) that PT inhibited recruitment of neutrophils to the lung and neutrophils played a role in antibody directed clearance of $B$. pertussis by the Fcy receptor ${ }^{57}$. As the dose of the aP decreased, we observed an increase in lung and blood neutrophils by the $1 / 160^{\text {th }}$ neutrophil percentages were similar to those of naïve $B$. pertussis challenged mice. It is 
likely that as the aP dose decreased, there was less PT neutralization due to a decrease in anti-PT titers. Lung neutrophil populations of mice immunized with inclusion of RTX into the $1 / 40^{\text {th }}$ and $1 / 80^{\text {th }}$ doses were significantly reduced $(p=0.0142$ and $p=$ 0.008 , respectively) compared to mice immunized with aP only at the same doses (Fig. $5 \mathrm{C}$ ); however, by day 3 , this reduction was no longer significant because neutrophil levels had returned to levels observed in non-challenged mice (Fig. 5D). We observed lower circulating neutrophil populations in the blood at both days when RTX was included compared to aP only doses (Fig. 5F) (1/40 th: $\left.p=0.018,1 / 80^{\text {th: }} p=0.071\right)$. Collectively, these data suggest that inclusion of RTX into the aP vaccine impacts the neutrophil populations in the respiratory tissues and circulating neutrophils in the blood.

Decreased macrophages in the respiratory tract due to inclusion of RTX into the aP.

Macrophages are a primary target of ACT and are readily killed by the toxin ${ }^{41,43}$. We hypothesized that as ACT is neutralized in vivo, that there would be an increase in macrophages due to their increased survival. However, our data did not support that hypothesis, and instead we saw that both RTX supplemented aP groups had lower macrophages in the nasal lavage at day 1 compared to aP only. Inclusion of RTX decreased the overall numbers of macrophages in both the $1 / 40^{\text {th }}$ and $1 / 80^{\text {th }}$ base aP groups (Fig. 6AB). At day 3 , we observed that mice vaccinated with $1 / 40^{\text {th }}, 1 / 80^{\text {th }}$ and $1 / 160^{\text {th }}$ aP showed increasing amounts of macrophages compared to naïve and naïve challenged mice. As observed for other parameters measured, this effect might be due to an induction of a naïve Th1 response due to insufficient aP driven Th2 as a result of our decrease in aP dose.

Visualizing and modeling the relationships between: antibodies against $B$. pertussis toxins, pro-inflammatory signals, phagocytes, and clearance of $B$. pertussis from the murine respiratory tract.

The Kendrick test was used to establish units of efficacy for whole cell vaccines in mice to estimate the dose to be used in humans ${ }^{2}$. The murine intranasal infection model was 
then developed to validate aP vaccines ${ }^{44,58}$. Since the introduction Tdap, numerous pre-clinical studies have been performed to evaluate new antigens or adjuvants to improve aPs. However, we argue that there is a need for standardization of the murine evaluation platform to facilitate the development of these formulations, and transition to other models such as the baboon. In this study, we titrated DTaP and found a dose $\left(1 / 80^{\text {th }}\right.$ human) that was partially protective with the $B$. pertussis strain (UT25) and the challenge dose of $2 \times 10^{7} \mathrm{CFU}$ that were used. It is clear that decreases in bacterial burden are indicative of protection; however, we aimed to visually represent all the data collected to support our conclusions. For this comparison, we represented the increases and decreases for each variable relative to the no vaccine / no challenge group using a 64 color scheme (Fig. 7). If the relative units increases, the color changes to red, or if it decreases then the value is represented in blue. We then plotted the average values per each immunization group $\left(1 / 20-1 / 160^{\text {th }}\right)$ in relation to the RTX supplemented groups. As one visually compares $1 / 80^{\text {th }}$ to $1 / 80^{\text {th }}+R T X$ at day 1 or 3 , it is apparent that adding RTX relatively decreases all values towards the green or no relative change, which we interpret as overall protection. It is also important to note that values at day 1 are relatively higher than day 3 and this relates to the fact that the total amount of bacteria are decreasing. The amount of anti-PT or anti-RTX is shown in black to grey to white color scale (Fig. 7). There is a clear correlation with amount of anti-PT and overall protection. In addition, it is interesting to see that inclusion of RTX enhanced the anti-PT levels in the $\mathrm{aP}+\mathrm{RTX}$ groups. Overall, variables associated with inflammation such as cytokines or phagocytes tend to return to green or naïve non-infected levels in mice vaccinated with aP+RTX. We only observed five relatively large decreases (indicated in blue). The $1 / 20^{\text {th }}$ aP group had relatively lower $\mathrm{KC} / \mathrm{GRO}$ which could be associated with the anti-inflammatory Th2 response due to the high amount of alum in the immunized mice. We also saw a relative decrease in number of macrophages present in the nasal wash at day 3 in the naïve group. This decrease could be attributed to high amounts of PT and ACT secreted during infection which could be impairing normal macrophage recruitment in the airway. We also saw large relative decreases in macrophages in the RTX supplemented $1 / 40^{\text {th }}$ and $1 / 80^{\text {th }}$ groups at day 3 and reasons for these changes were described above. Combining these data allow us to consider the overall 
inflammatory response in relation to the vaccination status, to further demonstrate the effects of including RTX into aP formulations, and to begin to establish a multi-variable approach to determining efficacy of experimental vaccines in the mouse.

\section{Discussion}

There is a resurgence of whooping cough that coincides with the switch to the use of aP vaccines. In this study, we aimed to accomplish two goals. First, we sought to define an $\mathrm{aP}$ vaccine dose, or doses, that allow the evaluation of synergy between the current aP and potential new antigens. The second goal was to evaluate RTX as an antigen for inclusion in acellular pertussis vaccines in the murine challenge model. As a result of these studies, we now better appreciate the need to continue to refine the methodologies of improving aP vaccines.

We initiated this study by evaluating the efficacy of ACT and RTX as single vaccine antigens. Immunization with ACT or RTX alone did not induce significant protection in CD-1 mice with the UT25 B. pertussis challenge strain (Fig. 1). The AC domain of ACT was first described as a protective antigen by Guiso et al ${ }^{17}$. In that study, the antigen preparation that was used consisted of two polypeptides of 43 and $45 \mathrm{kDa}$. We now know that these polypeptides corresponded to the AC domain of ACT. In those studies, BALB/c mice were vaccinated twice with $A C$ polypeptide absorbed on aluminum hydroxide adjuvant and it was observed that $80 \%$ of the AC-vaccinated mice survived $B$. pertussis challenge with strain $18323{ }^{17}$. The aforementioned study was then further supported by a subsequent study by the same group that showed the protective capacity of the AC domain and full length recombinant ACT protein ${ }^{35}$. Several more

studies showed that: 1) CyaC acylation of ACT was required for the protective activity ${ }^{18}$ and 2) that the C-terminal domain was also required for protection with recombinant ACT. Full length recombinant ACT was protective when $15 \mu \mathrm{g}$ of antigen were mixed and absorbed with $250 \mu \mathrm{g}$ of alum ${ }^{19}$. We used less antigen and adjuvant than Betsou et al. (either $10 \mu \mathrm{g}$ of ACT or $5.6 \mu \mathrm{g}$ of RTX with $125 \mu \mathrm{g}$ of alum) and we did not observe protection in CD-1 mice (Fig. 1A), and the use of the MPLA adjuvant did not improve bacterial clearance compared to alum. An apparent difference between our study and 
that of Betsou et al. is that strain 18323 was used, which has been suggested to be an outlier compared to most other global B. pertussis strains. Guiso et al. noted that strain 18323 produces less PT than the Tohama I type strain ${ }^{35}$. From our own studies, we know that UT25 produces more PT and ACT than Tohama I (data not shown). This information highlights the importance of the challenge strain. Another caveat of our study is that Betsou et al. absorbed the ACT antigen to alum, which we did not with RTX. It is possible that absorbing RTX to the aP could further enhance the synergistic protection observed in this study when RTX was combined with the alum-containing aP. Another key aspect to consider is the potential endotoxin contamination in recombinant ACT preparations. Villarino et al. used recombinant ACT but did not observe the same bacterial clearance as Betsou et al. ${ }^{19}$. ACT used by Cheung et al. was highly purified of endotoxin ${ }^{21}$, as was the RTX that we used in this study ${ }^{9}$. It is possible that endotoxin levels in ACT preparations may influence the overall protection afforded by such antigens as they may induce a more Th1-biased response.

Several of the aforementioned studies indicated that single antigen vaccines containing ACT were protective but the study by Cheung et al. and our RTX-only study (Fig. 1) were not in concordance. Other recent studies testing single antigen vaccines composed of proteins expressed during biofilm or in response to iron starvation showed that very few of these single antigen formulations were protective on their own ${ }^{27,46}$. Given that the aP antigens of either DTaP or Tdap (PT, FHA, PRN, and +/- FIM) do provide some protection against disease, we hypothesize that new antigens to be included in the aP should be tested not only alone, but also in combination with aP antigens. Only one study has investigated ACT as an antigen in combination with the aP in mice ${ }^{21}$. Cheung et al. reported that adding 12.5 or $25 \mu \mathrm{g}$ to $1 / 8^{\text {th }} \mathrm{aP}$, significantly decreased bacterial burden in mice at 7 days post challenge with strain $1832{ }^{21}$. PT and FHA are formulated in the DTaP (INFANRIX®) at $25 \mu \mathrm{g}$ each in 1 human dose. Therefore, the use of $25 \mu \mathrm{g} \mathrm{ACT}$ is roughly equal to 1 human dose of antigen (assuming no adjustment for molar mass of each antigen). We chose to test the utility of RTX antigen with lower antigen doses in an effort to best model what would be scaled towards a commercial vaccine dose. To do so, we identified a partially protective dose 
of aP, so synergistic effects of addition of the antigen to be tested. We titrated the aP vaccine from $1 / 20^{\text {th }}$ to $1 / 160^{\text {th }}$ human dose. As decreasing aP doses were tested, we saw an increase in innate immune responses to levels observed in naïve challenged mice (Fig. 7). From the bacterial burden in the lung, we determined that $1 / 40^{\text {th }}$ dose of aP is protective, while $1 / 80^{\text {th }}$ is only partially protective. However, it is important to note that $1 / 40^{\text {th }}$ aP immunization was non-protective in the nares at day 1 , but at that dose, B. pertussis was cleared by day 3 (Fig. 2EF). These data show that the protective capacity of an aP dose in the mouse is dependent on the location in the airway and the time elapsed since $B$. pertussis challenge. Using total clearance in all locations, $1 / 40^{\text {th }}$ aP immunization results in sterilizing immunity against our challenge dose as observed at three days post challenge. Based on these data, we selected the $1 / 40^{\text {th }}$ (minimal effective dose) and the $1 / 80^{\text {th }}$ (maximal non-effective dose) to supplement with $A C$ toxoid antigen (RTX).

We identified the $1 / 80^{\text {th }}$ aP dose that would allow us to tease out RTX antigen effects that would otherwise be masked by the use of a high mouse to human aP dose (e.g. $\left.1 / 5^{\text {th }}\right)$. With the $1 / 80^{\text {th }}$ aP dose, it is clear that innate responses are occurring due to the lack of protection provided by the immunization. We hypothesize that decrease in innate responses would correlated with induction of antibody production that either: neutralizes the effects of the pathogen (e.g. toxins: PT or ACT) or results in killing of the pathogen (e.g. opsonization, phagocytosis, or complement killing). We were able to detect antibodies that recognized RTX (Fig. 3AB) and we saw that adding RTX enhanced production of anti-PT (Fig. $3 \mathrm{~A}$ ). When we supplemented the $1 / 80^{\text {th }}$ aP dose with RTX, we were able to observe positive effects on the response to $B$. pertussis challenge such as decreased: bacterial burden (Fig 2B), IL-6 secretion (Fig. 4A), IFN-Y (Fig. 4E), IL-5 (Fig. S4), IL-2 (Fig. S4). We speculate that the neutralization of ACT in vivo leads to a decrease in the innate immune responses at day 1 post challenge, but at day 3 , the levels of these pro-inflammatory cytokine were not affected by the inclusion of RTX (Fig. 4BF and S4). We observed that $B$. pertussis challenge increased neutrophil levels in the nasal lavage and that these levels were not reduced to non-challenged levels during our study (Fig. 5AB). Neutrophils in both the nares and lungs were overall decreased by day 
3 post challenge in the RTX supplemented groups. Adding RTX antigen to the $1 / 40^{\text {th }}$ and $1 / 80^{\text {th }}$ vaccines decreased the total neutrophils in the lungs at day 1 and same trend was observed for blood neutrophils (Fig. 5EF). Interestingly, we observed that adding RTX to aP (1/40 th nor $\left.1 / 80^{\text {th }}\right)$ did not affect nasal neutrophils amounts (Fig. 5AB). We speculate that ACT may have specific effects in the lung and not in the nares of mice, but more experiments are necessary test this hypothesis.

ACT can induce apoptosis in macrophages ${ }^{59}$ and it is also known that IL-12 production by macrophages plays a role in vaccine-mediated clearance of $B$. pertussis ${ }^{60}$. We measured the number of macrophages in the lungs and nasal lavages of aP or RTX supplemented aPs and saw less macrophages when RTX was added to the aP (Fig. $6 A B C D)$. This data was somewhat counterintuitive but Skopova et al. have shown that CAMP signaling and hemolysin activity synergize and provoke PMN and macrophage influx into the lungs ${ }^{12}$. Our macrophage observations suggest anti-RTX may be neutralizing ACT in vivo. There are also two more possibilities: 1) there may be less macrophage recruitment because there are less bacteria or 2) if macrophages are less impaired (due to ACT neutralization), they may be effectively migrating to the lymph nodes to present antigens. At this point, we do not have sufficient data to fully understand the relationships between the amount of macrophages in the airway and if that is an indication of protection and/or an effect of ACT neutralization.

$B$. pertussis strains have evolved in response to aP immunization ${ }^{61}$. Pertactin is no longer expressed by circulating $B$. pertussis strains ${ }^{62}$ and recently, a $B$. pertussis strain was isolated and sequenced that lacked PT ${ }^{63}$. B. parapertussis is not capable of expressing $\mathrm{PT}^{64}$ but causes as many as $\sim 15 \%$ of whooping cough cases. $B$. holmesii is an underappreciated pathogen that cause whooping cough-like illness and does not express $\mathrm{PT}{ }^{65}$. In addition, there are unpublished reports of strains sequenced by the $\mathrm{CDC}$ that lack expression of filamentous hemagglutinin. Given the potential rise of triple mutant strains that do not make pertactin, FHA, or PT, it is possible that the efficacy of aP protection will further decline if strains arise that lack the main antigens that most aP vaccines contain. Based on these possibilities, we propose that adding additional antigens that are both immunogenic and important for bacterial virulence is necessary 
to slow the progression of $B$. pertussis evolution ${ }^{66}$, and the resistance to aP-mediated immunity.

We aimed to investigate ACT as a vaccine antigen and we developed a new evaluation approach involving analysis of multiple correlates of protection (Fig. 7). Our data suggest that RTX antigen may enhance protection of aP vaccines, based on what we observed in CD-1 mice. The different protection levels observed in each of the regions of the airway (nares, trachea, and lungs) suggest that it may also be advantageous to consider the development of mucosal $B$. pertussis vaccines. This would allow for the pathogen to be neutralized rapidly in the upper airway before infection is fully established when highly potent PT and ACT are impairing the innate and adaptive immune responses. Higgs et al. ${ }^{67}$ proposed that aP vaccines containing antigens that direct a more diverse response, may be more effective. While we have focused on adding RTX to a Th2 inducing aP, it would be very interesting to formulate and test RTX containing vaccines with Th1 adjuvants. Neutralization of ACT along with a cellular response would likely be an ideal protection profile and possibly address the waning protection issue of the aP. Our study was focused on RTX but there are other candidates that should be evaluated as well. Our study highlights the importance of titrating the vaccine dose and we expect this will provide new methodologies for the development of pertussis vaccines. Significant research is still necessary to formulate and evaluate safe and more effective next generation pertussis vaccines, which could include ACT as an antigen.

\section{Funding Information}

D.T.B was funded by a West Virginia Space Grant Consortium Graduate Student Fellowship and the Jennifer Gossling Fellowship. This project has been funded in part with Federal funds from the National Institute of Allergy and Infectious Diseases, National Institutes of Health, Department of Health and Human Services, under Contract No. HHSN272201200005C. Additional support was provided by, NIH/NIAID grant 5 RO1 Al1018000 [E.L.H.], NIH/NIAID grant RO1 A1122753 [J.A.M], and laboratory startup funds from West Virginia University to F.H.D. Flow Cytometry experiments were performed in the West Virginia University Flow Cytometry Core 
Facility, which is supported by the National Institutes of Health equipment grant number S10OD016165 and the Institutional Development Award (IDeA) from the National Institute of General Medical Sciences of the National Institutes of Health under grant numbers P30GM103488 (COBRE) and P20GM103434 (INBRE).

\section{Acknowledgements}

We would like to thank Dr. Kathleen Brundage for assistance with flow cytometry and Dr. Josh Eby of University of Virginia for critical discussions that contributed to this project. 


\section{Figures}

Figure 1. RTX only immunization does not protect against $B$. pertussis challenge in CD-1 mice nor does including RTX with $1 / 5^{\text {th }}$ aP improve clearance kinetics but ACT neutralizing antibodies are produced.
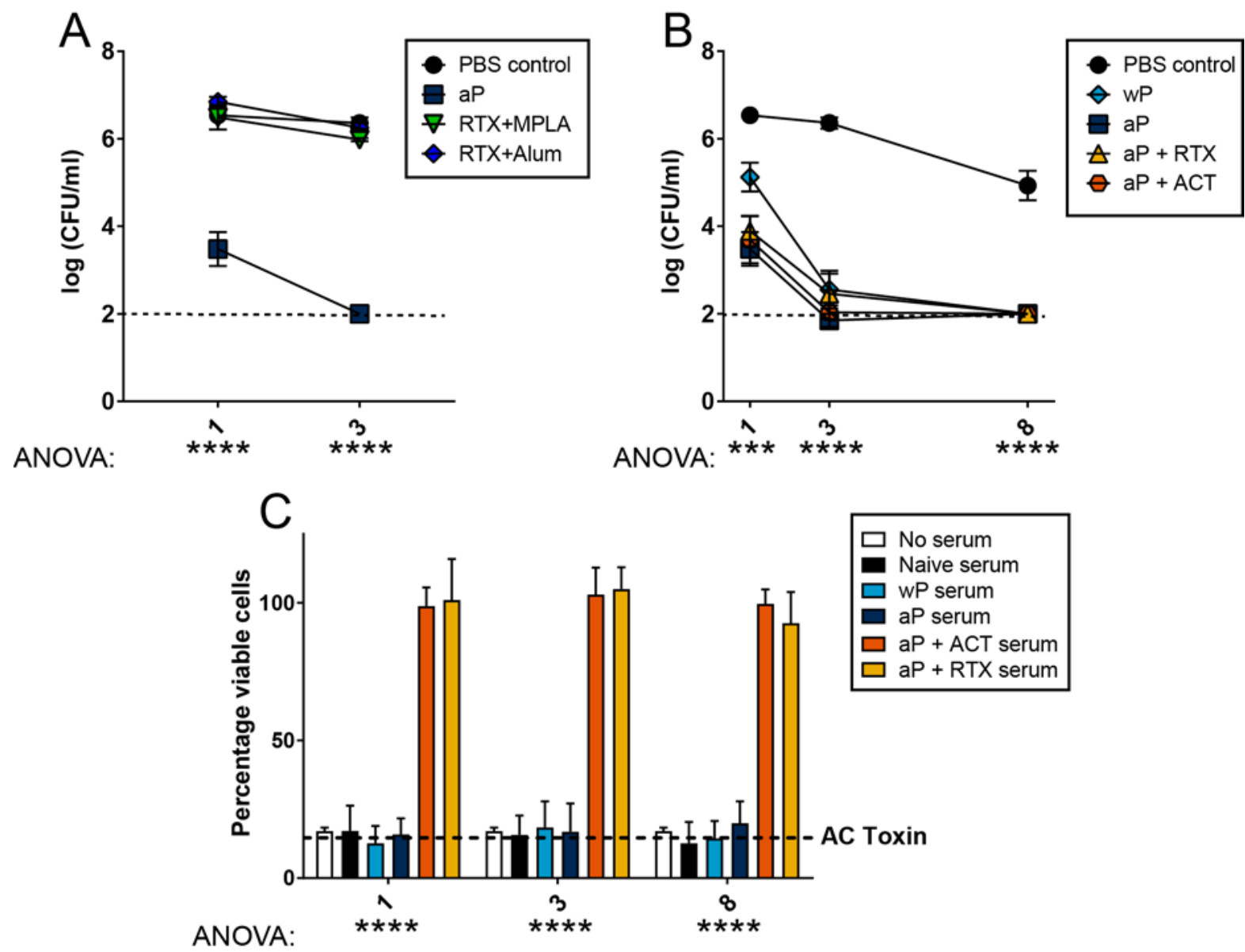

Fig

ure 1. RTX only immunization does not protect against $B$. pertussis challenge in CD-1 mice nor does including RTX with $1 / 5^{\text {th }}$ aP improve clearance kinetics but ACT neutralizing antibodies are produced. (A) CD-1 mice were vaccinated with $P B S$ vehicle, $1 / 5^{\text {th }}$ human dose of aP, RTX $(5.6 \mu \mathrm{g})+$ MPLA $(20 \mu \mathrm{g})$, RTX $(5.6 \mu \mathrm{g})+$ Alum $(125 \mu \mathrm{g})$, or PBS as control vehicle (naïve)( $\mathrm{n}=4$, with 4 technical replicates). Mice were IN challenged with $2 \times 10^{7} B p$ (UT25Sm1). At days 1 and $3 \mathrm{pc}$ the bacterial burdens were determined by culturing of homogenates of the lungs on BG agar. The dashed line represents the lower limit of detection due to plating. Bacterial loads in mice vaccinated with aP were significantly lower than the PBS control group at day $1(p=<0.0001)$ and day 3 ( $p=<0.0001)$ in the lung. (B) CD-1 mice were vaccinated with PBS control vehicle, $\mathrm{wP}, \mathrm{aP}, \mathrm{aP}+\mathrm{ACT}$ or $\mathrm{aP}+\mathrm{RTX}$ and challenged intranasally with UT25Sm1 ( $n=4$, with 4 technical replicates). Mice vaccinated with $\mathrm{aP}, \mathrm{aP}+\mathrm{ACT}$, and $\mathrm{aP}+\mathrm{RTX}$ had a significantly lower bacteria burden than the PBS control group at day $1(p<0.0003, p$ 
$<0.0006, p<0.0013)$ in the lung. Bacterial loads in all immunized mice were significantly lower than PBS group at day 3 and $8(p<0.0001$; all groups) in the lung. Data in each group were compared to every other group at day 1, 3, or 8 pc using one-way ANOVA with Tukey's post hoc test. (C) Serum from vaccinated/challenged mice was used to determine if ACT neutralizing antibodies were produced following RTX or ACT inclusion in aP vaccine by adenylate cyclase toxin neutralization assay. J774A.1 macrophages were cultured with active ACT, and serum from mice. Neutralization of ACT results in increase of macrophage survival. Serum from vaccinated mice with $\mathrm{AP}+\mathrm{ACT}$ and $\mathrm{aP}+\mathrm{RTX}$ resulted in neutralization of $\mathrm{ACT}$, compared to unvaccinated, $\mathrm{aP}$, and wP groups. Results are shown as mean \pm SEM, ${ }^{\star \star \star \star}, p<0.0001{ }^{* \star \star} p<0.001$ by one-way ANOVA. 
Figure 2. Respiratory tract bacterial burden of CD-1 mice immunized with aP vaccines or aPs supplemented with RTX antigen.
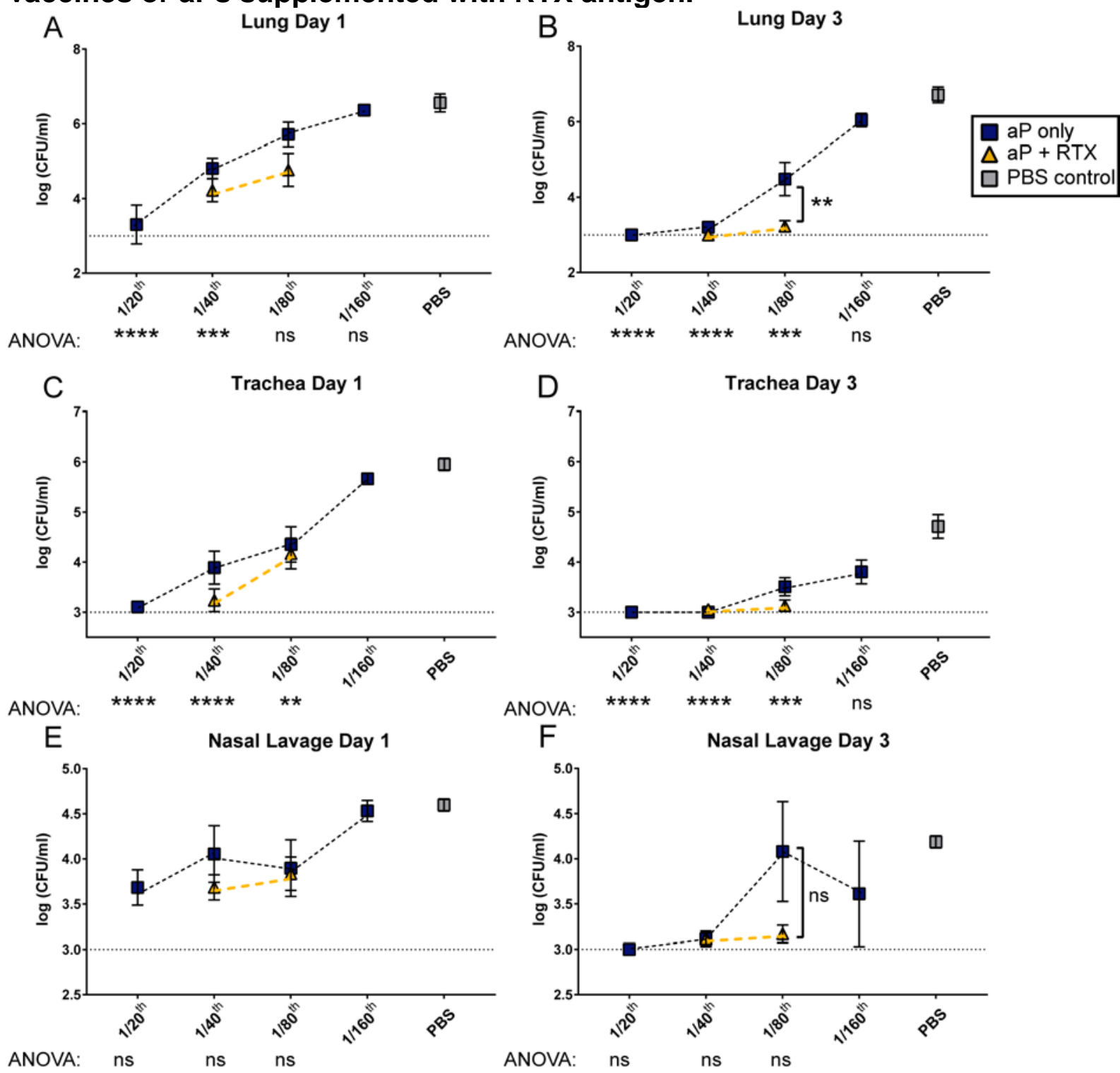

Figure 2. Respiratory tract bacterial burden of CD-1 mice immunized with aP vaccines or aPs supplemented with $R T X$ antigen. Immunization with $1 / 20^{\text {th }}, 1 / 40^{\text {th }}$, $1 / 80^{\text {th }}$, and $1 / 160^{\text {th }}$ of human dose aP was used to titrate the minimal effective dose and the maximum non-effective dose. RTX $(5.6 \mu \mathrm{g})$ was included into $1 / 40^{\text {th }}$ and $1 / 80^{\text {th }} \mathrm{aP}$ doses. Vaccinated CD-1 mice were challenged with $2 \times 10^{7} \mathrm{Bp}$, and bacterial burden was determined at days 1 and 3. CFU counts were determined from (AB) lung homogenate, (CD) trachea homogenate, and nasal lavage (EF). Results are mean \pm SEM ( $n=4-8$, with 4 technical replicates) from independent experiments, $* \star * *, p<0.0001$ ${ }^{\star * \star} p<0.001,{ }^{* \star} p<0.01,{ }^{*} p<0.05$ determined by one-way ANOVA with Tukey post hoc test compared to naïve mice (PBS control). The dashed line represents the lower limit of 
detection due to plating. The blue boxes indicate aP groups whereas aP+RTX are indicated by gold triangles. Gray squares indicate mock vaccinated, and challenged mice. In $E$ and $F$ there were no statistically significant comparisons between the aP titration doses as determined by ANOVA. 
Figure 3: Serological responses from mice immunized with aP titrations or aPs supplemented with RTX.
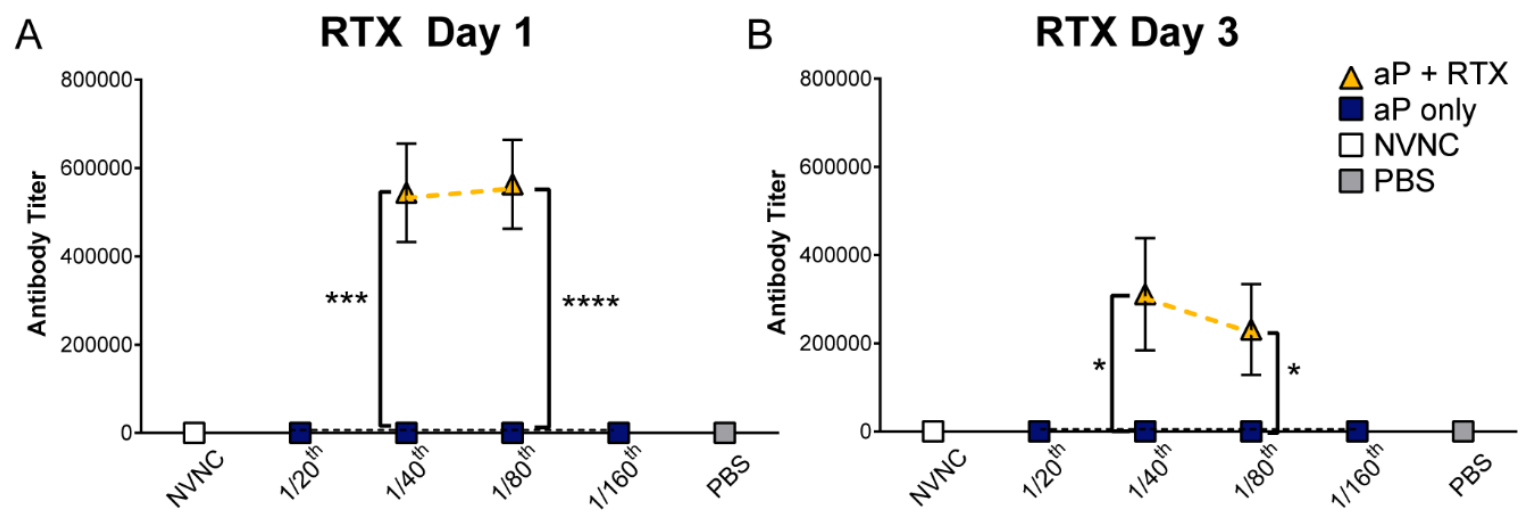

ANOVA: ns ns ns ns ns

ANOVA: ns ns ns ns ns
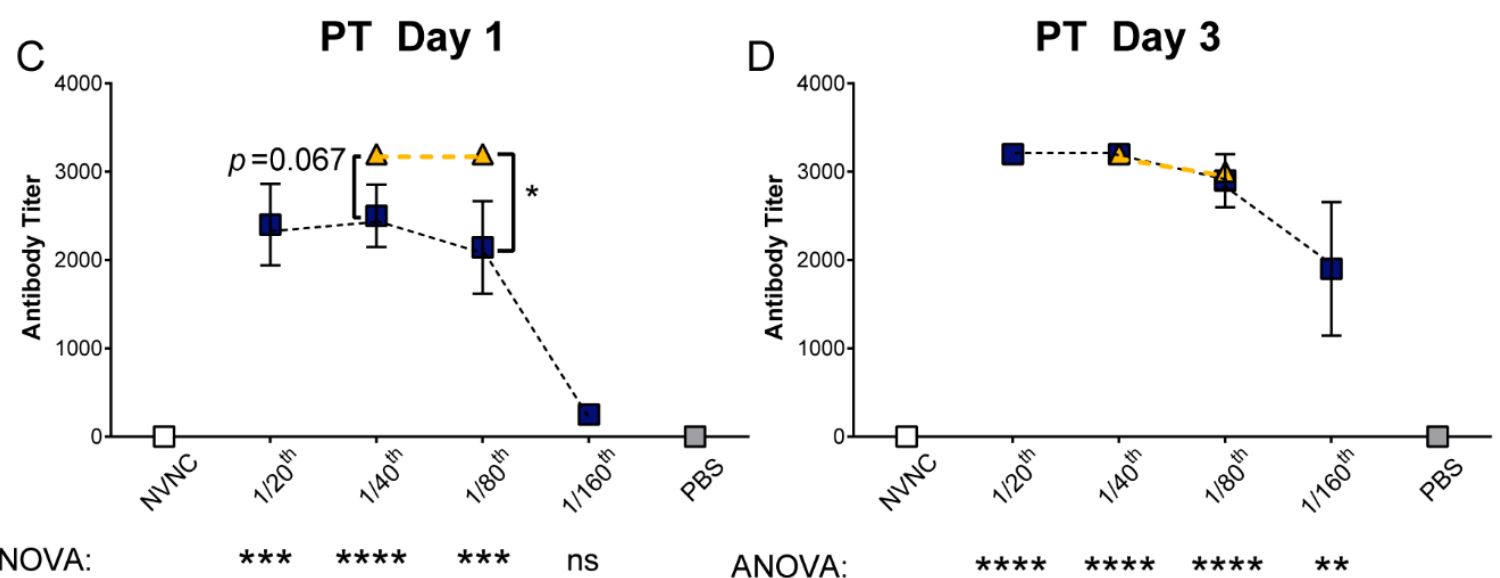

Figure 3: Serological responses from mice immunized with aP titrations or aPs supplemented with RTX. (AB) ELISA was used to determine the anti-RTX IgG titers in serum of immunized and challenged mice at 1 and 3 days pc. (CD) Anti-PT IgG titers in serum of immunized and challenged mice at indicated time points. Titrations of aP were compared by one-way ANOVA with Tukey's post hoc test, shown below graphs and compared to PBS group. Unpaired two-way T test was used to compare addition of RTX to aP titrations, shown by brackets. Results plotted on graph are mean $\pm \mathrm{SEM}$, ${ }^{* \star *} \mathrm{p}<$ $0.001{ }^{* *} p<0.01,{ }^{*} p<0.05$. 
Figure 4: Analysis of proinflammatory cytokines from lungs of mice immunized with aP titrations or aPs supplemented with RTX.

A

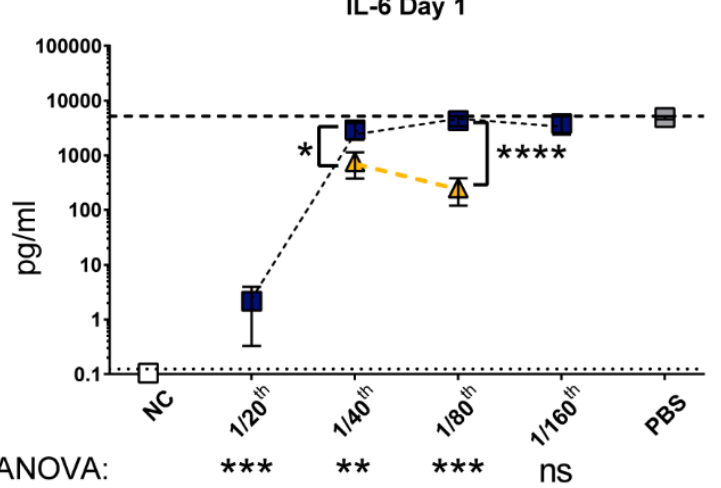

C

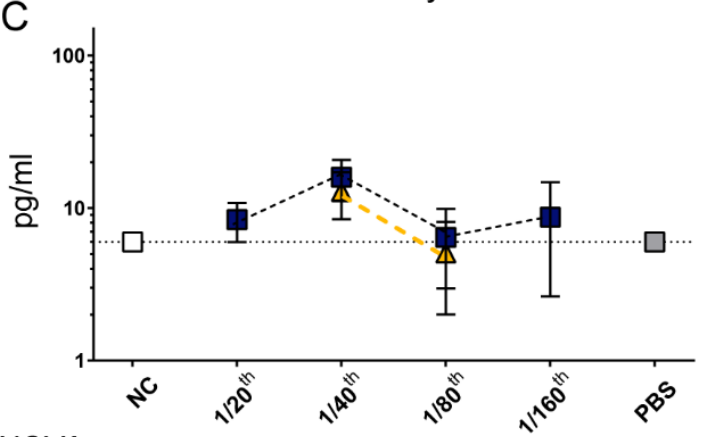

ANOVA:

$\mathrm{E}$

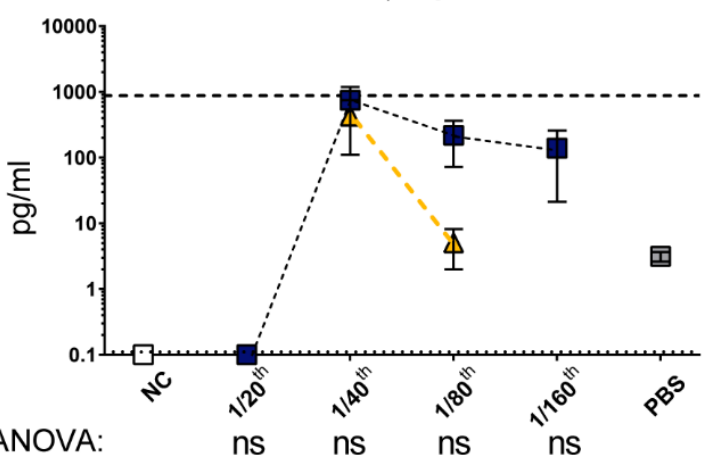

G

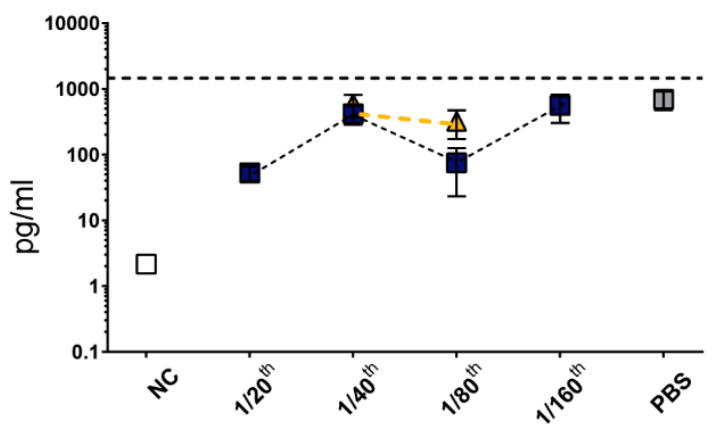

ANOVA:
IL-17 Day 1

ns ns ns

IFN- $\gamma$ Day 1

IL-1 $\beta$ Day 1

ns ns ns ns
B

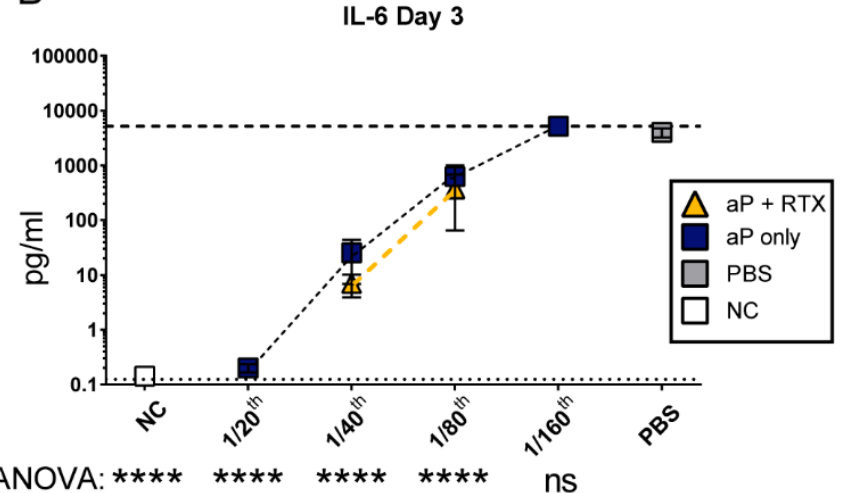

IL-17 Day 3

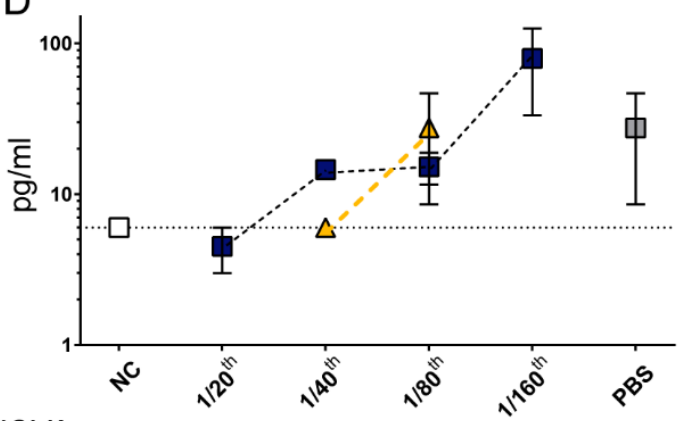

ANOVA: ns ns ns ns

$\mathrm{F}$

IFN- $\gamma$ Day 3

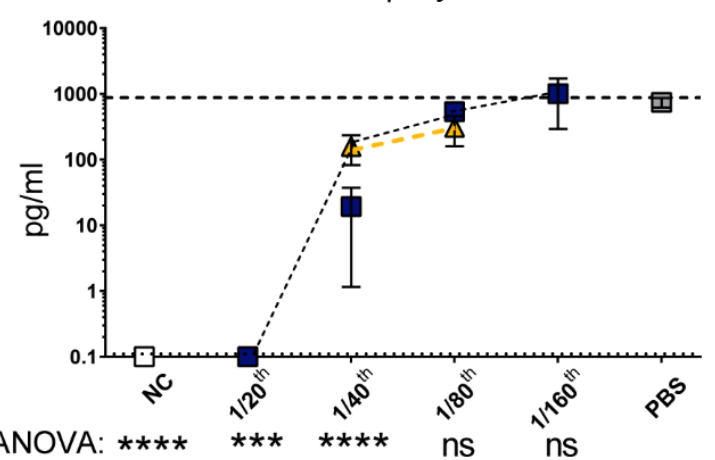

$\mathrm{H}$

IL-1 $\beta$ Day 3

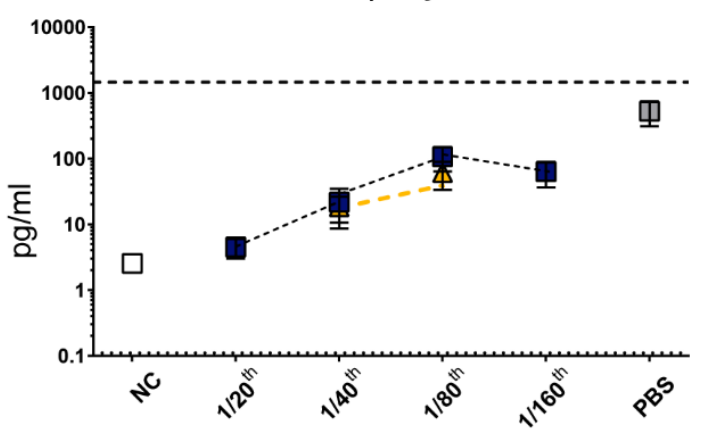

ANOVA: * * * ns ns 
Figure 4: Analysis of proinflammatory cytokines from lungs of mice immunized with aP titrations or aPs supplemented with RTX. Th1 associated cytokines from supernatant of lung homogenates analyzed at days 1 and 3 pc. Cytokines (AB) IL-6, (CD) IL-17, (EF) IFN- $\gamma$, and (GH) IL-1 $\beta$. The blue boxes indicate aP groups whereas $\mathrm{aP}+\mathrm{RTX}$ are indicated by gold triangles. Gray squares indicate mock vaccinated, and challenged mice, while white squares represent non-vaccinated and non-challenged mice (NC). Results are mean \pm SEM, ${ }^{* \star \star *} p<0.0001$, ${ }^{* \star *} p<0.001,{ }^{*} p<0.05$ by one-way ANOVA with Tukey post hoc test compared to PBS (naïve) but challenged mice. Brackets between two points represent unpaired two-way $T$ test. Dashed line indicates upper limits of detection, and dotted line indicates lower limit of detection. 
Figure 5: Flow cytometric analysis of blood, pulmonary, and nasal lavage neutrophils from mice immunized with aP titrations or aPs supplemented with RTX.
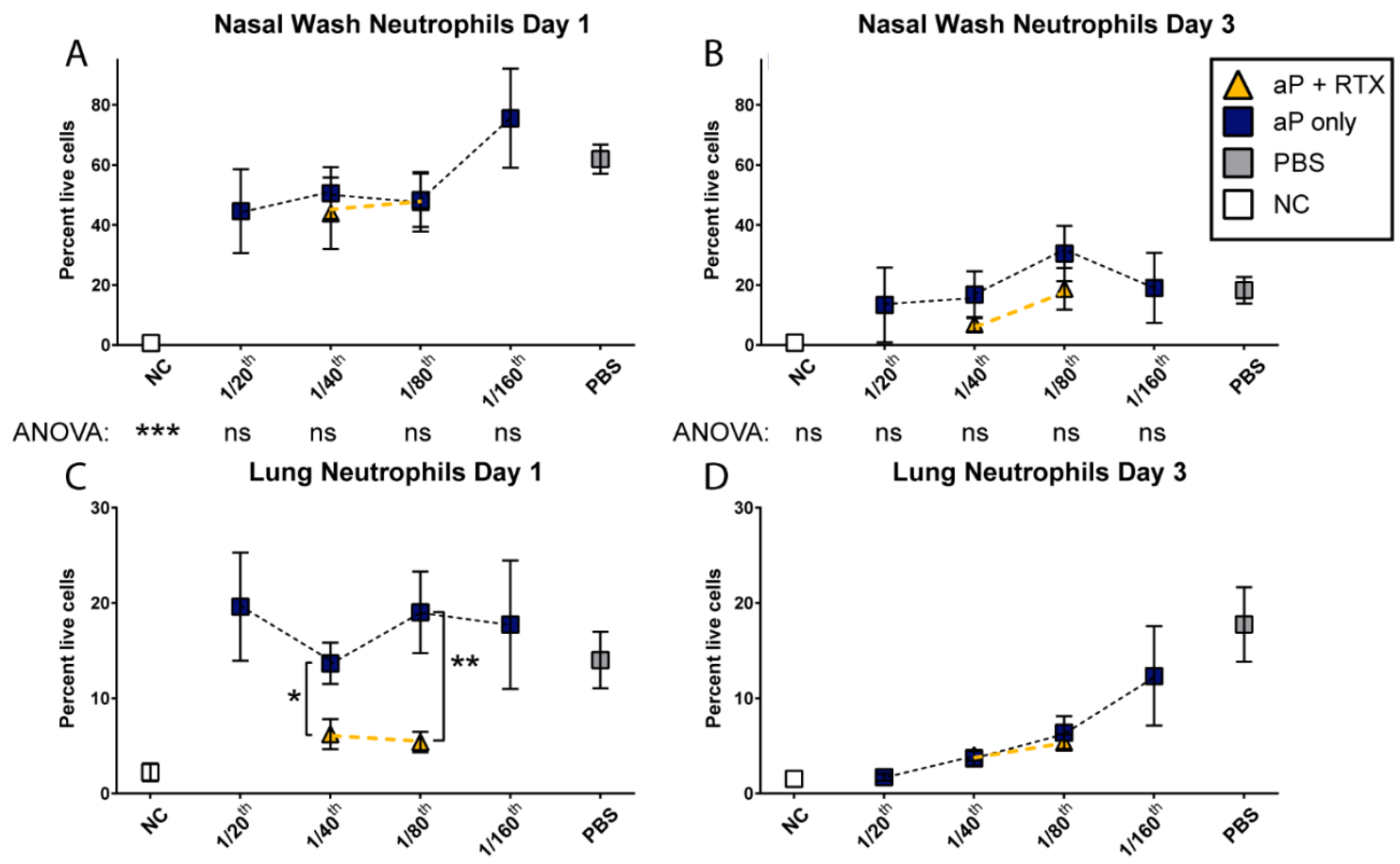

ANOVA: ns ns ns ns ns
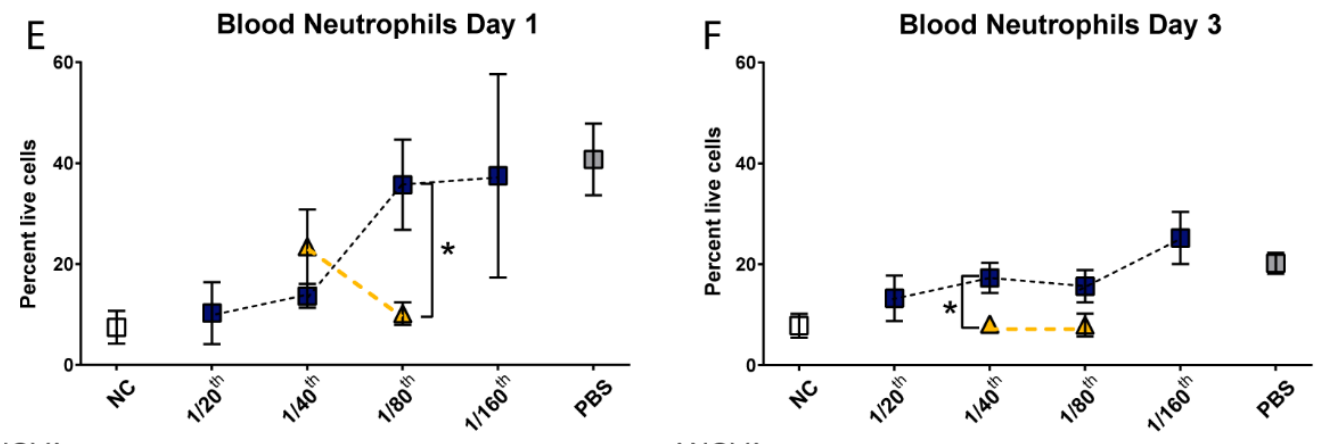

ANOVA: ns ns ns ns ns

ANOVA: ns ns ns ns ns

Figure 5: Flow cytometric analysis of blood, pulmonary, and nasal lavage neutrophils from mice immunized with aP titrations or aPs supplemented with RTX. Quantification of the percentage of live, single cells that were classified as neutrophils (GR-1 ${ }^{\text {hi }} C D-11 b^{+}$) from (AB) nasal lavage, (CD) lung homogenate, or (EF) blood. Results are mean \pm SEM, ${ }^{* \star *} p<0.001,{ }^{* *} p<0.01,{ }^{*} p<0.05$ by one-way ANOVA with Tukey post hoc test compared to PBS (naïve) but challenged mice. Brackets between two points represent unpaired two-way $T$ test. 
Figure 6: Flow cytometric analysis of macrophages from lung and nasal lavage of mice immunized with aP titrations or aPs supplemented with RTX.
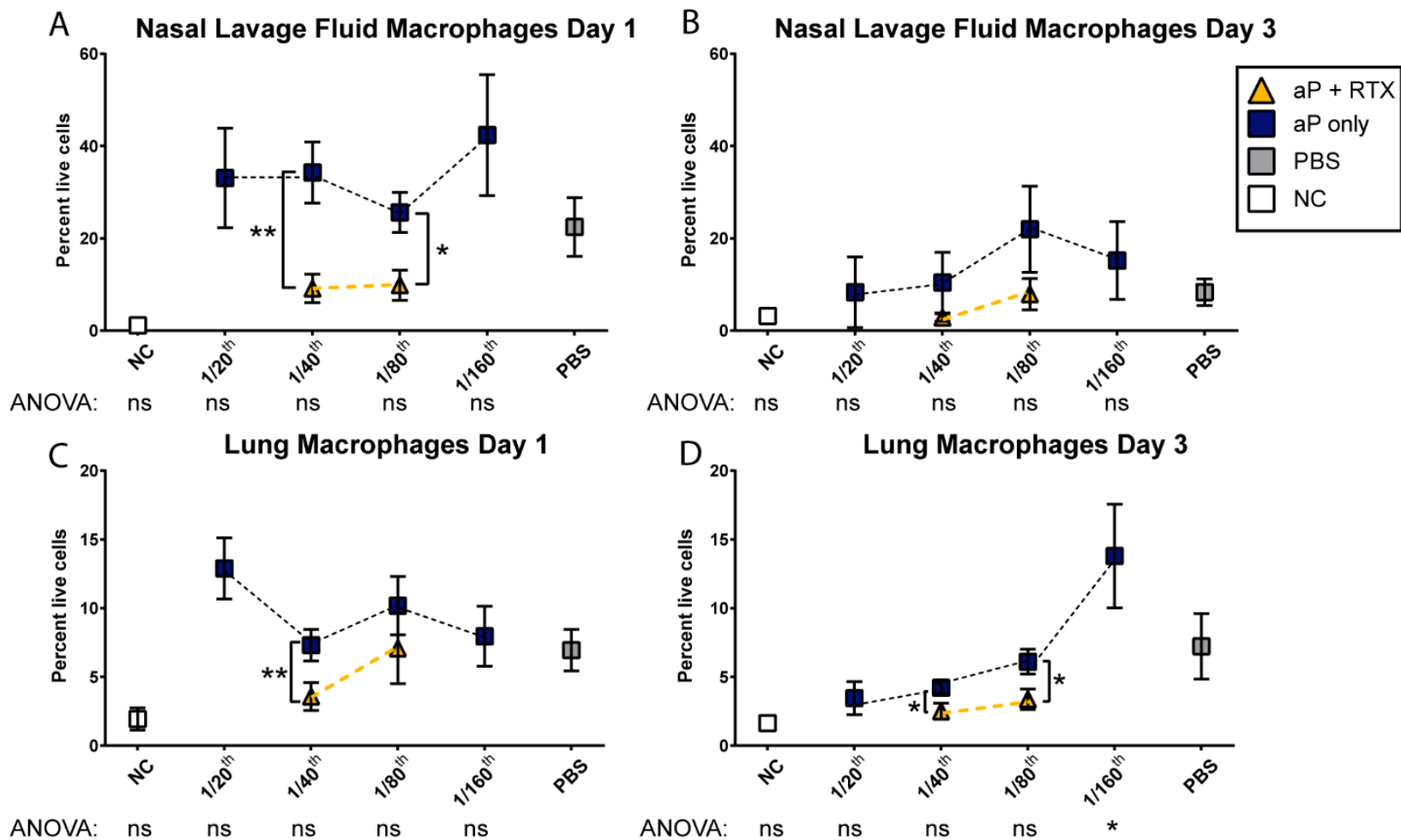

ANOVA: ns ns ns ns ns

ANOVA:

Figure 6: Flow cytometric analysis of macrophages from lung and nasal lavage of mice immunized with aP titrations or aPs supplemented with RTX. Percentage of macrophages (defined as $C D-11 b^{+} F 4 / 80^{+}$) from live, single cells from $(A B)$ nasal lavage and $(C D)$ single cell suspension prepared from lung homogenate. Results shown are mean \pm SEM, ${ }^{*} p<0.05$ by one-way ANOVA with Tukey post hoc test compared to PBS (naïve) but challenged mice. Brackets between two points represent unpaired two-way T test. 


\section{Tables}

\section{Table 1: Composition of vaccines for Fig. 1}

TABLE 1 Compositions of vaccines used for Fig. 1

\begin{tabular}{|c|c|c|c|c|c|c|}
\hline \multirow[b]{2}{*}{ Vaccine component } & \multicolumn{6}{|c|}{ Value for vaccine group ${ }^{a}$ : } \\
\hline & RTX + alum & RTX + MPLA & aP (1/5 human dose) & $a P+A C T$ & $a P+R T X$ & wPb \\
\hline Pertussis toxoidc & 0 & 0 & 5 & 5 & 5 & 0.4 \\
\hline Filamentous hemagglutinin & 0 & 0 & 5 & 5 & 5 & 3.5 \\
\hline Pertactin & 0 & 0 & 1.6 & 1.6 & 1.6 & 0.3 \\
\hline Adenylate cyclase toxin or RTX & 5.6 & 5.6 & 0 & 10 & 5.6 & 0.2 \\
\hline Aluminum hydroxide & 0 & 125 & 125 & 125 & 125 & 0 \\
\hline Other antigens/adjuvants & 0 & 20 & 0 & 0 & 0 & 62 \\
\hline
\end{tabular}

aAll masses of antigens or adjuvant are indicated in $\mu \mathrm{g}$.

${ }^{b}$ This estimate is based on the number of peptides identified by mass spectrometry for each antigen in the wP (data not shown). The percentage is then used to estimate the potential mass based on the fact that the WP dose used in this study contained $66 \mu \mathrm{g}$ of total protein.

cFor total pertussis toxin, the sum of PtxA, $-B,-C$, and $-D$ peptides was combined. 
Table 2: Composition of vaccines used in the titration of aP and RTX supplementation studies

TABLE 2 Compositions of vaccines used in the titration of aP and RTX supplementation studies for Fig. 2 to 6

\begin{tabular}{lllllll}
\hline & \multicolumn{7}{l}{ Value for vaccine group ${ }^{a}$ : } \\
\cline { 2 - 7 } Vaccine component & $\mathbf{1 / 2 0} \mathrm{aP}$ & $\mathbf{1 / 4 0} \mathrm{aP}$ & $\mathbf{1 / 4 0} \mathrm{aP}+\mathrm{RTX}$ & $\mathbf{1 / 8 0} \mathrm{aP}$ & $\mathbf{1 / 8 0} \mathrm{aP}+\mathrm{RTX}$ & $1 / 160 \mathrm{aP}$ \\
\hline Pertussis toxoid & 1.25 & 0.63 & 0.63 & 0.33 & 0.33 & 0.16 \\
Filamentous hemagglutinin & 1.25 & 0.63 & 0.63 & 0.33 & 0.33 & 0.16 \\
Pertactin & 0.4 & 0.2 & 0.2 & 0.1 & 0.1 & 0.05 \\
Adenylate cyclase toxin or RTX & 0 & 0 & 5.6 & 0 & 5.6 & 0 \\
Aluminum hydroxide & 31.25 & 15.63 & 15.63 & 7.81 & 7.81 & 3.91 \\
\hline
\end{tabular}

${ }^{a}$ All masses of antigens or adjuvant are indicated in $\mu \mathrm{g}$. 


\section{Supplemental Figures}

Figure S1. RTX only nor RTX supplemented aP immunizations alter $B$. pertussis clearance in CD1 mice.
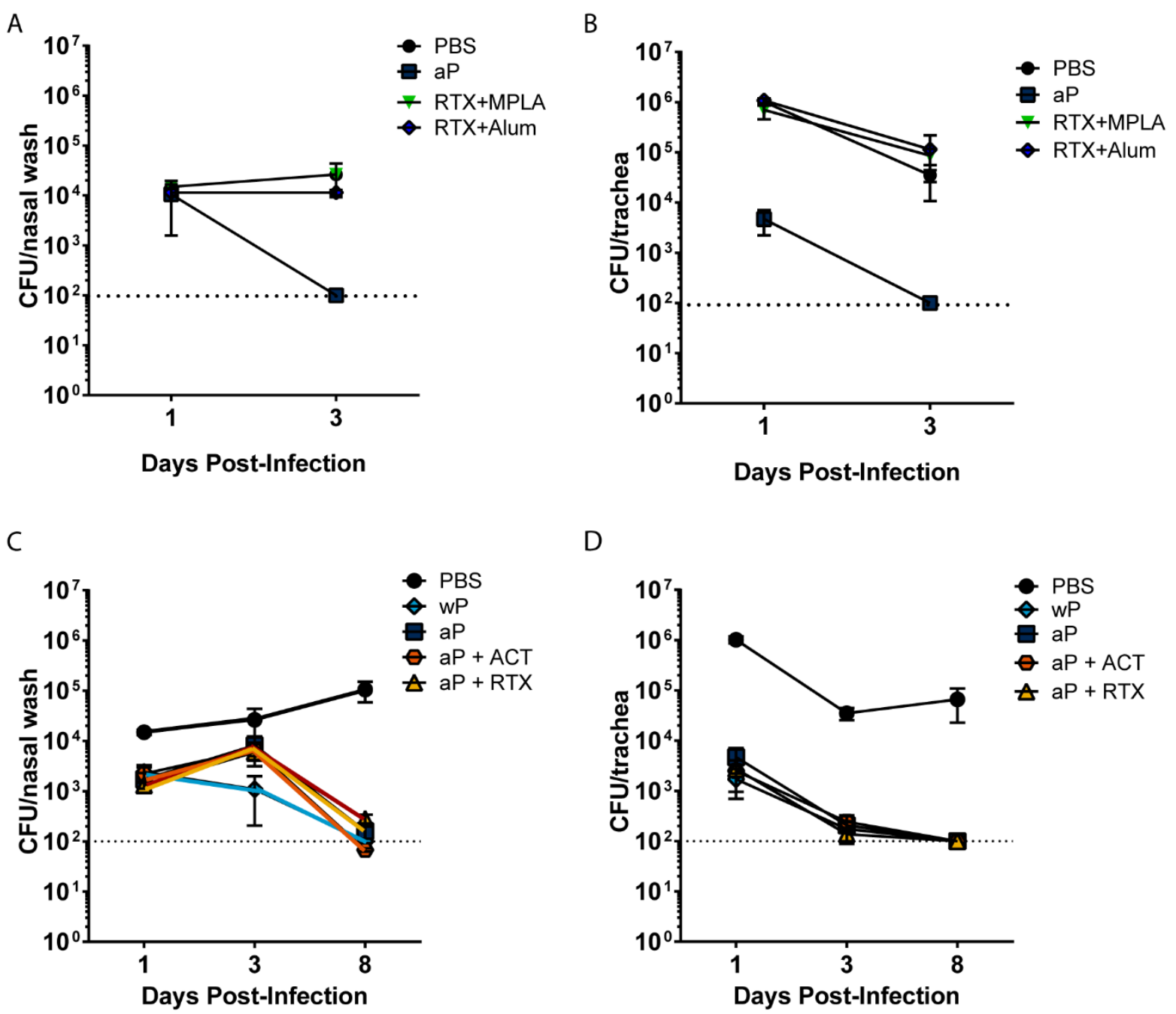

Figure S1. RTX only nor RTX supplemented aP immunizations alter $B$. pertussis clearance in CD1 mice. (A-B) CD1 mice were vaccinated with PBS vehicle, aP, RTX adjuvanted with alum or MPLA and challenged intranasally with $B$. pertussis strain UT25. At days 1 and 3 post challenge the bacterial burdens were determined by culturing of nasal wash, homogenates of trachea on Bordet Gengou (BG) agar. Experiments were performed with 4 mice per group with 4 technical replicates. The dashed line represents the lower limit of detection. (CD) CD1 mice were vaccinated with PBS control vehicle, wP, aP, aP + ACT or aP + RTX and challenged intranasally with UT25. At days 1,3 , and 8 post challenge the bacterial burdens were determined. Experiments were performed with 4 mice per group with 4 technical replicates. 
Figure S2. Mouse inflammatory cytokine array of lung homogenate supernatant.

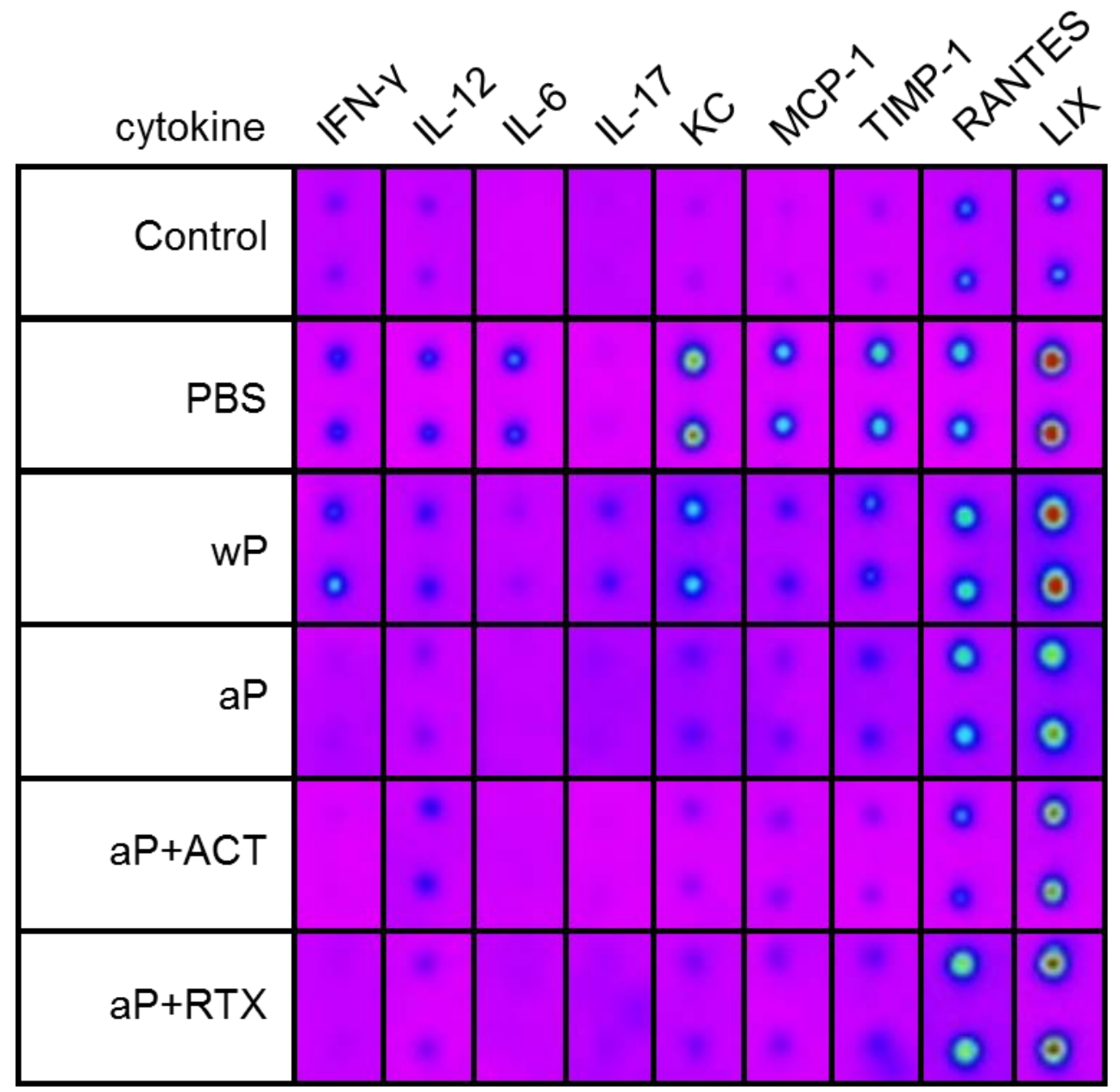

Figure S2. Mouse inflammatory cytokine array of lung homogenate supernatant. Lung homogenate supernatant from 4 mice in each group were pooled and used to probe membranes labelled with antibodies for 40 inflammatory cytokines. Pseudocolor image of day 3 cytokine production are shown in duplicate. The control group is not challenged with $B$. pertussis. 
Figure S3. Cytokine analysis of lung homogenates of CD1 mice immunized with various aP doses and supplemented with RTX antigen.
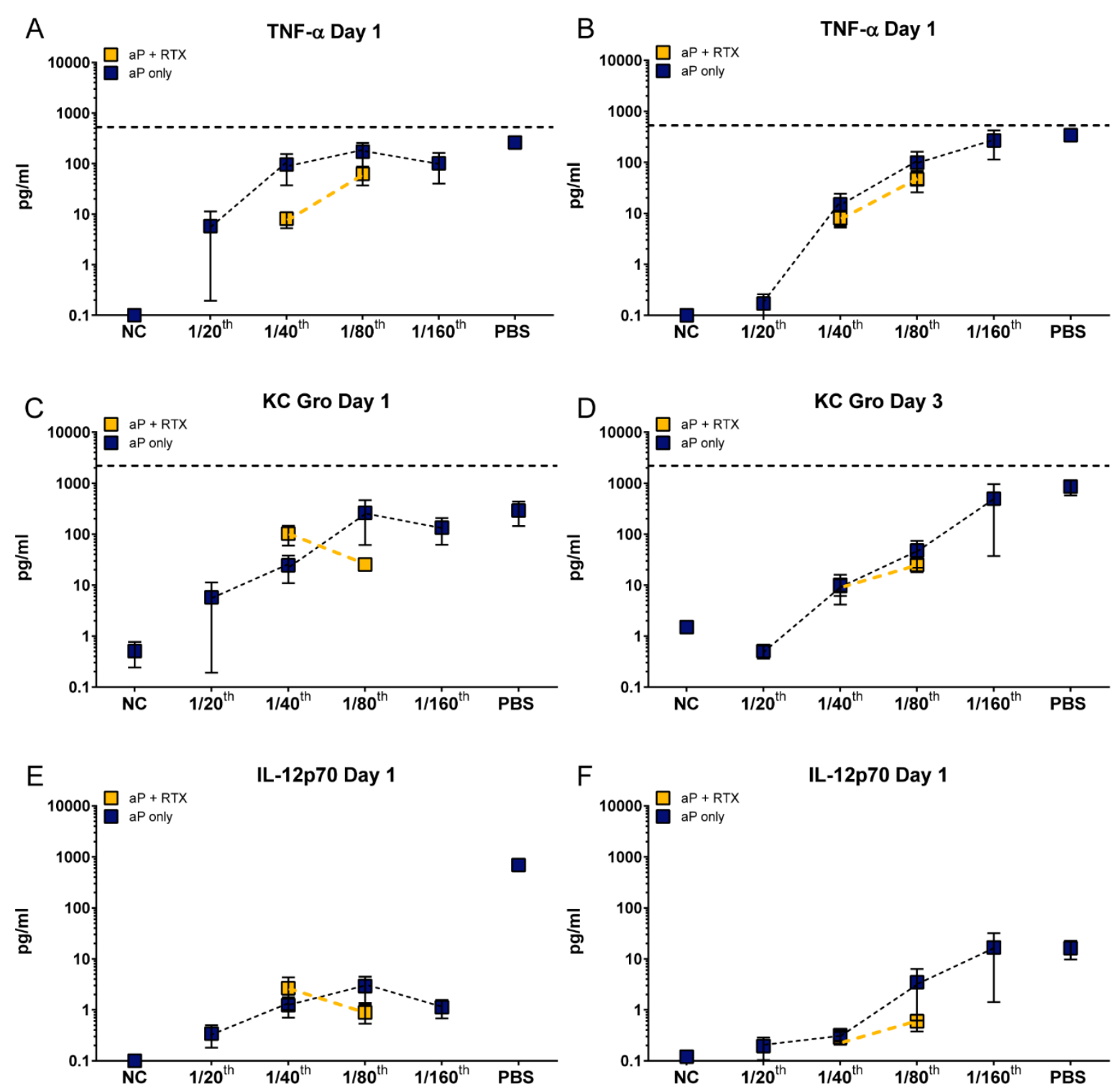

Figure S3. Cytokine analysis of lung homogenates of CD1 mice immunized with various aP doses and supplemented with RTX antigen. Lung homogenates were collected from mice of each of the groups indicated and the concentration of each cytokine indicated was determined. Minimal detection limits are indicated. Error bars show standard error of the mean. The blue boxes indicate aP groups whereas aP+RTX are indicated in gold. 
Figure S4. Cytokine analysis of lung homogenates of CD1 mice immunized with various aP doses and supplemented with RTX antigen.
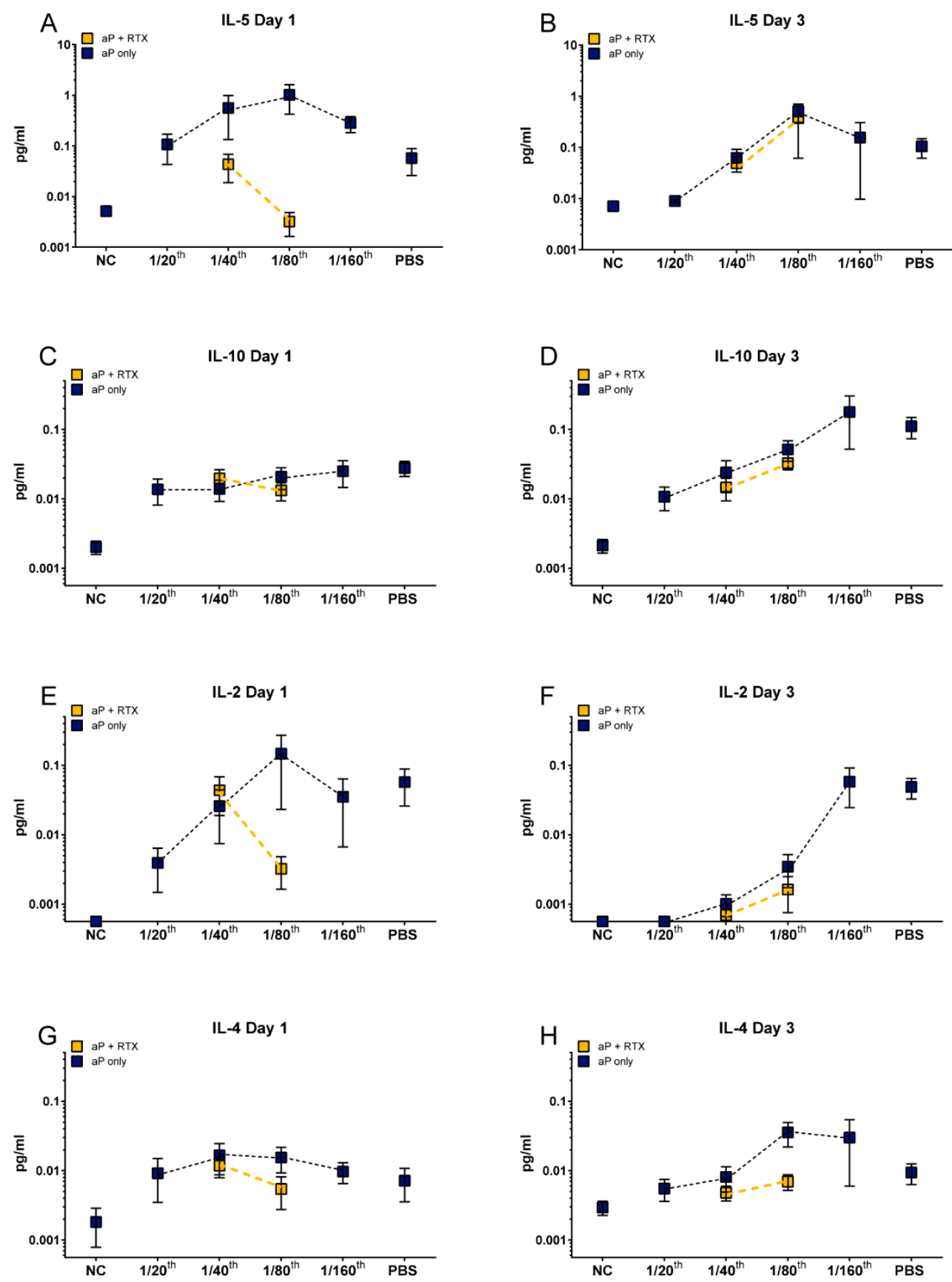

Figure S4. Cytokine analysis of lung homogenates of CD1 mice immunized with various aP doses and supplemented with RTX antigen. Lung homogenates were collected from mice of each of the groups indicated and the concentration of each cytokine indicated was determined. Minimal detection limits are indicated. Error bars 
show standard error of the mean. The blue boxes indicate aP groups whereas aP+RTX are indicated in gold.

Figure S5: Serological responses from mice immunized with aP titrations or aPs supplemented with RTX.
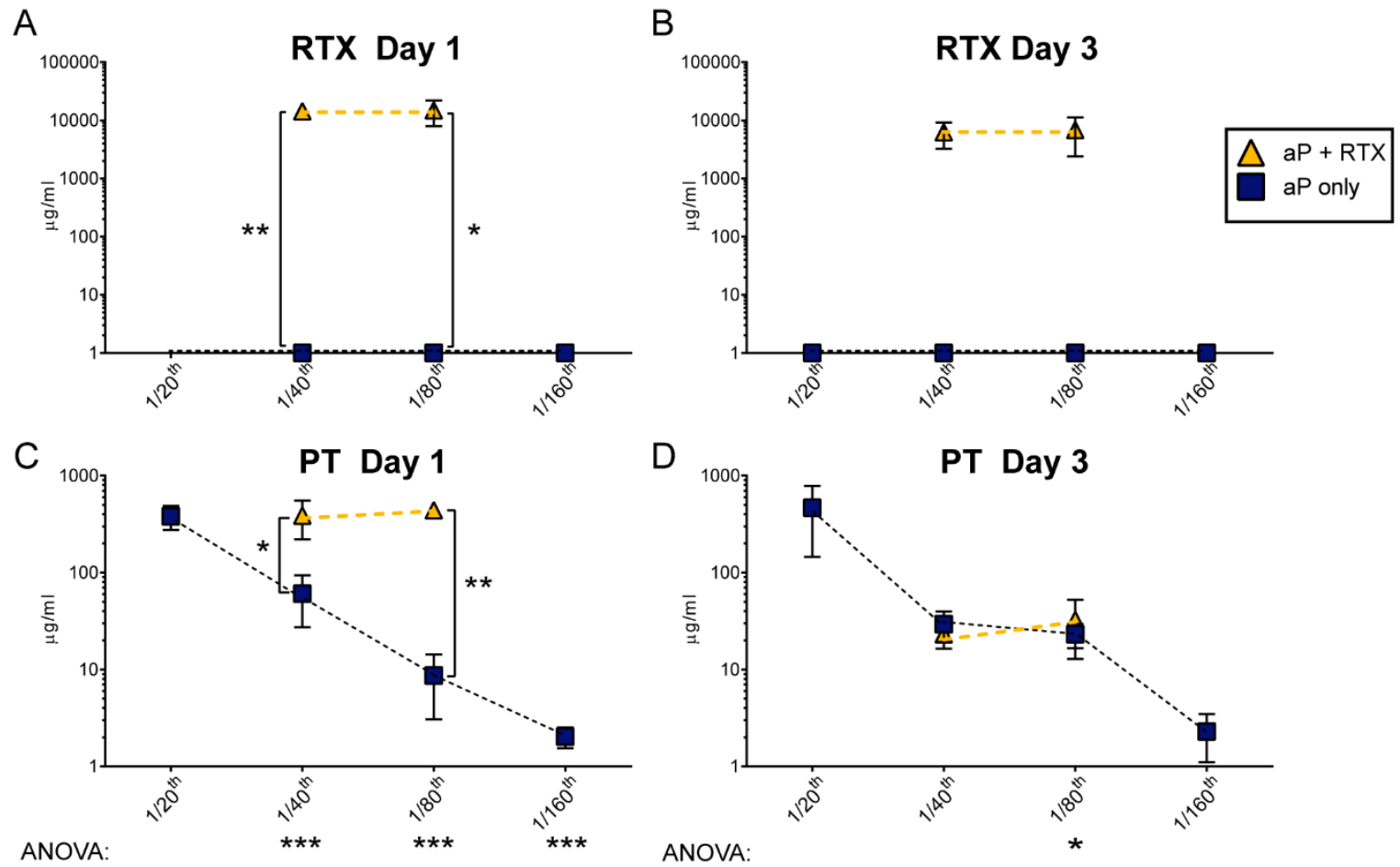

Figure S5: Serological responses from mice immunized with aP titrations or aPs supplemented with RTX. (AB) ELISA was used to determine the anti-RTX IgG levels in serum of immunized and challenged mice at 1 and 3 days pc. (CD) Anti-PT IgG levels in serum of immunized and challenged mice at indicated time points. Antibody concentration $(\mu \mathrm{g} / \mathrm{ml})$ represented were determined using relative EC50. Serum absorbance values were logarithmically plotted to known antibody standards using a four-parameter logistic curve. Relative EC50 was then determined by fitting serum concentration to dilutions of samples using a four-parameter logistic curve. Titrations of aP were compared by one-way ANOVA with Tukey's post hoc test, shown below graphs. Unpaired two-way T test was used to compare addition of RTX to aP tritrations, shown by brackets. Results plotted on graph are mean \pm SEM, ${ }^{\star \star \star} p<0.001{ }^{* \star} p<0.01$, ${ }^{*} \mathrm{p}<0.05$. 
Figure S6

A

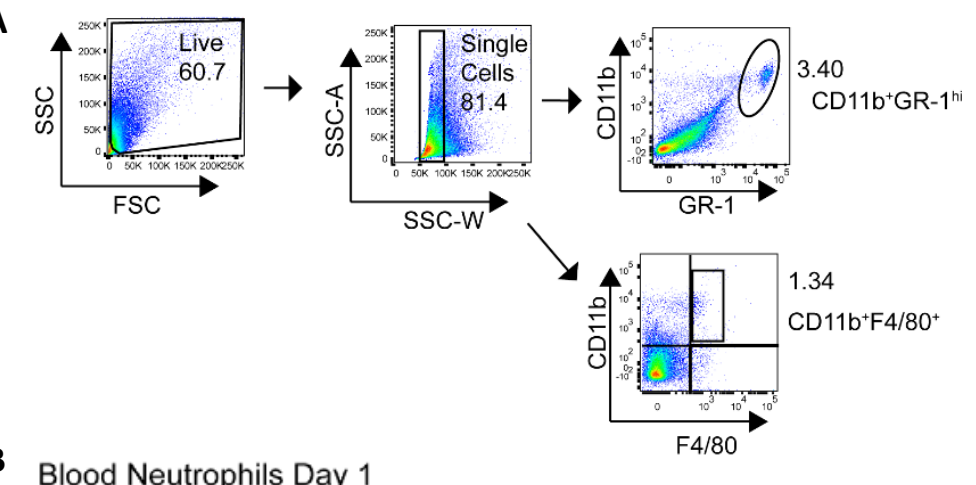

Blood Neutrophils Day 1

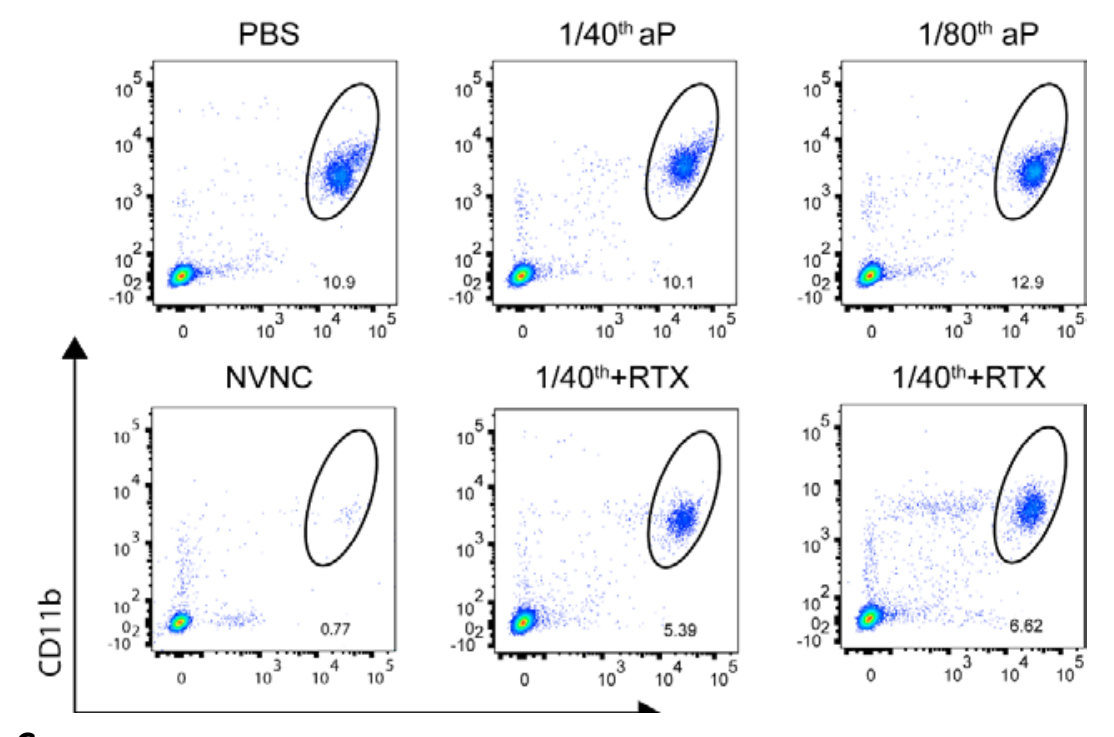

C Lung Macrophages Day 3
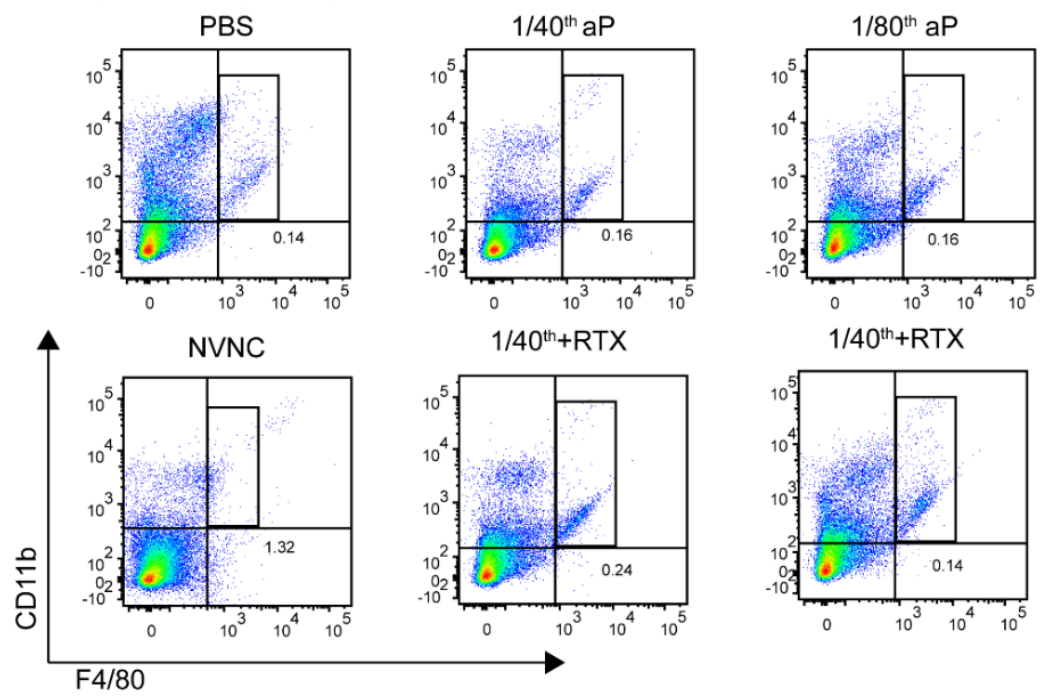
Figure S6. Representative images and gating strategy for neutrophils from blood of CD1 mice immunized with various aP doses and supplemented with RTX antigen. (A) Cells were first gated on live, single cells. Then classified as either (B) neutrophils $\left(\mathrm{CD} 11 \mathrm{~b}^{+} \mathrm{Gr}-\mathrm{H}^{\mathrm{Hi}}\right)$ or $(\mathrm{C})$ macrophages $\left(\mathrm{CD} 11 \mathrm{~b}^{+} \mathrm{F} 4 / 80^{+}\right)$. Quadrants demonstrate positively labeled cells determined by single label controls marker flow cytometry controls. Region of interest shows neutrophil population $\left(\mathrm{Gr}-1^{+} \mathrm{CD} 11 \mathrm{~b}^{+}\right)$. 
Figure S7. Heat map modeling of the effects of base aP dose and supplementation of RTX antigen on correlates of protection in mice.

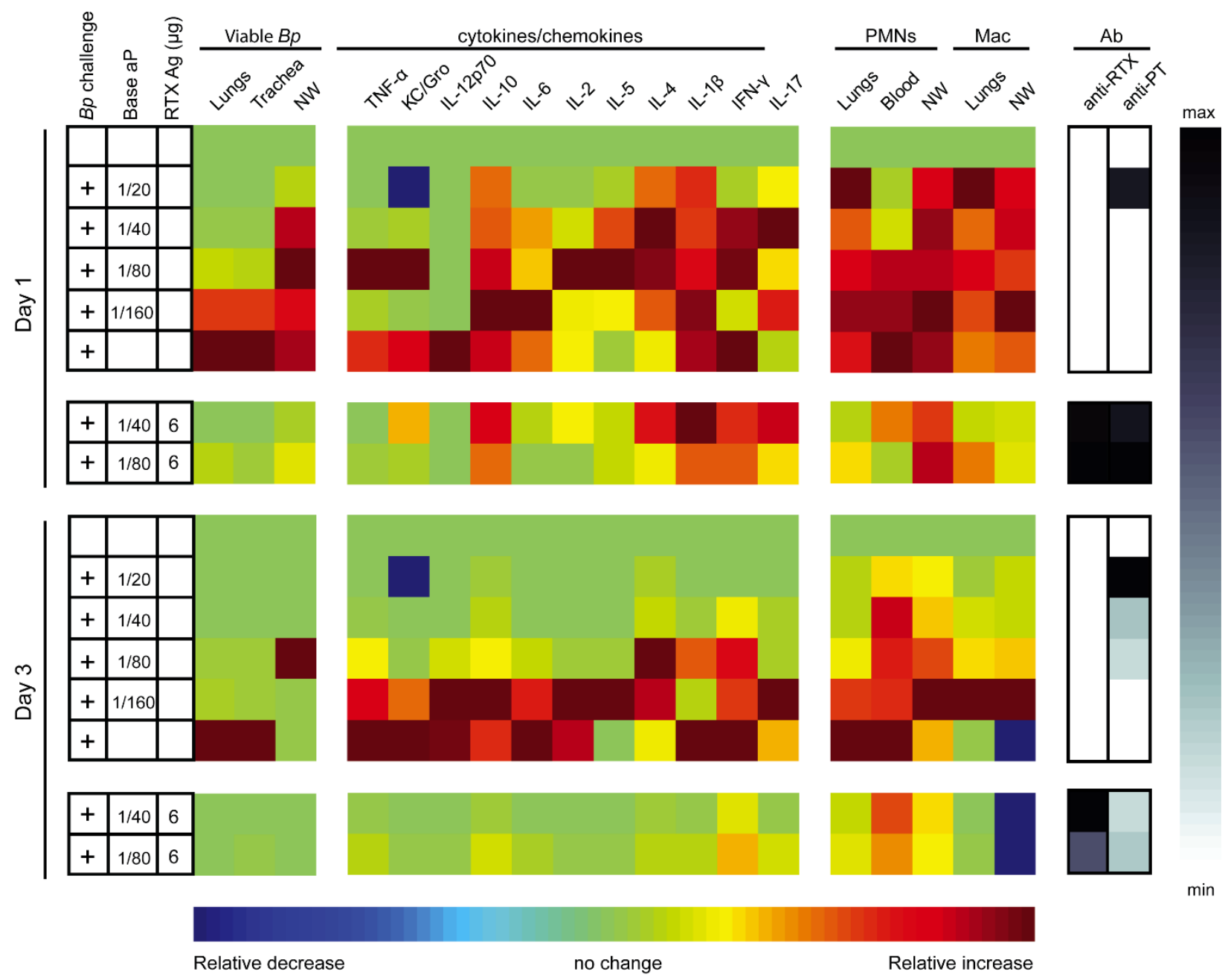

Figure S7. Heat map modeling of the effects of base aP dose and supplementation of RTX antigen on correlates of protection in mice. In order to understand the key variables collected in this study, in relation to base aP dose and the effects of adding RTX, we developed a heat map that shows the relative increase or decrease of each variable. The color scale indicates relative changes in bacterial burden, cytokines, or phagocytes whereas the black-grey-white scale indicates relative toxin specific antibodies in the serum of each group. 


\section{References}

1 Madsen, T. Vaccination against whooping cough. Journal of the American Medical Association 101, 187-188, doi:10.1001/jama.1933.02740280007003 (1933).

2 Kendrick, P. L., Eldering, G., Dixon, M. K. \& Misner, J. Mouse Protection Tests in the Study of Pertussis Vaccine: A Comparative Series Using the Intracerebral Route for Challenge. American journal of public health and the nation's health 37, 803-810 (1947).

3 Sato, Y. \& Sato, H. Development of acellular pertussis vaccines. Biologicals 27, 61-69, doi:10.1006/biol.1999.0181S1045-1056(99)90181-1 [pii] (1999).

4 Cherry, J. D. Why Do Pertussis Vaccines Fail? Pediatrics 129, 968-970, doi:10.1542/peds.2011-2594 (2012).

$5 \quad$ Klein, N. P., Bartlett, J., Fireman, B., Rowhani-Rahbar, A. \& Baxter, R. Comparative effectiveness of acellular versus whole-cell pertussis vaccines in teenagers. Pediatrics 131, e1716-1722, doi:10.1542/peds.2012-3836 (2013).

6 Klein, N. P., Bartlett, J., Rowhani-Rahbar, A., Fireman, B. \& Baxter, R. Waning protection after fifth dose of acellular pertussis vaccine in children. N Engl J Med 367, 1012-1019, doi:10.1056/NEJMoa1200850 (2012).

7 Warfel, J. M., Zimmerman, L. I. \& Merkel, T. J. Acellular pertussis vaccines protect against disease but fail to prevent infection and transmission in a nonhuman primate model. Proc Natl Acad Sci U S A 111, 787-792, doi:10.1073/pnas.1314688110 (2014).

8 Sebo, P., Osicka, R. \& Masin, J. Adenylate cyclase toxin-hemolysin relevance for pertussis vaccines. Expert review of vaccines 13, 1215-1227, doi:10.1586/14760584.2014.944900 (2014).

9 Wang, X., Maynard, J. A., Hewlett, E. L. \& Maynard, J. A. The Bordetella adenylate cyclase repeat-in-toxin (RTX) domain is immunodominant and elicits neutralizing antibodies. J Biol Chem 290, 3576-3591, doi:10.1074/jbc.M114.585281 (2015).

10 Ehrmann, I. E., Gray, M. C., Gordon, V. M., Gray, L. S. \& Hewlett, E. L. Hemolytic activity of adenylate cyclase toxin from Bordetella pertussis . FEBS letters 278, 79-83 (1991).

11 Khelef, N., Sakamoto, H. \& Guiso, N. Both adenylate cyclase and hemolytic activities are required by Bordetella pertussis to initiate infection. Microb Pathog 12, 227-235, doi:0882-4010(92)90057-U [pii] (1992).

12 Skopova, K. et al. Cyclic AMP-Elevating Capacity of Adenylate Cyclase ToxinHemolysin Is Sufficient for Lung Infection but Not for Full Virulence of Bordetella pertussis . Infect Immun 85, doi:10.1128/IAI.00937-16 (2017). 
13 Eby, J. C. et al. Quantification of the adenylate cyclase toxin of Bordetella pertussis in vitro and during respiratory infection. Infect Immun 81, 1390-1398, doi:10.1128/IAI.00110-13 (2013).

14 Cherry, J. D. et al. Determination of serum antibody to Bordetella pertussis adenylate cyclase toxin in vaccinated and unvaccinated children and in children and adults with pertussis. Clin Infect Dis 38, 502-507, doi:10.1086/381204 (2004).

15 Farfel, Z. et al. Antibodies to Bordetella pertussis adenylate cyclase are produced in man during pertussis infection and after vaccination. Journal of medical microbiology 32, 173-177, doi:10.1099/00222615-32-3-173 (1990).

16 Hewlett, E. L., Manclark, C. R. \& Wolff, J. Adenyl cyclase in Bordetella pertussis vaccines. J Infect Dis 136 Suppl, S216-219 (1977).

17 Guiso, N., Rocancourt, M., Szatanik, M. \& Alonso, J. M. Bordetella adenylate cyclase is a virulence associated factor and an immunoprotective antigen. Microb Pathog 7, 373-380, doi:0882-4010(89)90040-5 [pii] (1989).

18 Betsou, F., Sebo, P. \& Guiso, N. CyaC-mediated activation is important not only for toxic but also for protective activities of Bordetella pertussis adenylate cyclase-hemolysin. Infect Immun 61, 3583-3589 (1993).

19 Betsou, F., Sebo, P. \& Guiso, N. The C-terminal domain is essential for protective activity of the Bordetella pertussis adenylate cyclase-hemolysin. Infect Immun 63, 3309-3315 (1995).

20 Hormozi, K., Parton, R. \& Coote, J. Adjuvant and protective properties of native and recombinant Bordetella pertussis adenylate cyclase toxin preparations in mice. FEMS immunology and medical microbiology 23, 273-282 (1999).

21 Cheung, G. Y. et al. Effect of different forms of adenylate cyclase toxin of Bordetella pertussis on protection afforded by an acellular pertussis vaccine in a murine model. Infect Immun 74, 6797-6805, doi:10.1128/IAI.01104-06 (2006).

22 Gray, M. C., Donato, G. M., Jones, F. R., Kim, T. \& Hewlett, E. L. Newly secreted adenylate cyclase toxin is responsible for intoxication of target cells by Bordetella pertussis . Mol Microbiol 53, 1709-1719, doi:10.1111/j.13652958.2004.04227.xMMI4227 [pii] (2004).

23 Glaser, P. et al. The calmodulin-sensitive adenylate cyclase of Bordetella pertussis : cloning and expression in Escherichia coli. Mol Microbiol 2, 19-30 (1988).

24 Hewlett, E. L., Gordon, V. M., McCaffery, J. D., Sutherland, W. M. \& Gray, M. C. Adenylate cyclase toxin from Bordetella pertussis . Identification and purification of the holotoxin molecule. J Biol Chem 264, 19379-19384 (1989).

25 Hewlett, E. L. et al. Adenylate cyclase toxin from Bordetella pertussis . Conformational change associated with toxin activity. J Biol Chem 266, 1750317508 (1991). 
26 Wang, X. \& Maynard, J. A. The Bordetella Adenylate Cyclase Repeat-in-Toxin (RTX) Domain Is Immunodominant and Elicits Neutralizing Antibodies. The Journal of Biological Chemistry 290, 3576-3591, doi:10.1074/jbc.M114.585281 (2015).

27 de Gouw, D. et al. Proteomics-identified Bvg-activated autotransporters protect against Bordetella pertussis in a mouse model. PLoS One 9, e105011, doi:10.1371/journal.pone.0105011 (2014).

28 Armstrong, M. E., Loscher, C. E., Lynch, M. A. \& Mills, K. H. IL-1beta-dependent neurological effects of the whole cell pertussis vaccine: a role for IL-1-associated signalling components in vaccine reactogenicity. Journal of neuroimmunology 136, 25-33 (2003).

29 Brickman, T. J. \& Armstrong, S. K. The ornithine decarboxylase gene odc is required for alcaligin siderophore biosynthesis in Bordetella spp.: putrescine is a precursor of alcaligin. J Bacteriol 178, 54-60 (1996).

30 Bordet J \& O, G. Le microbe de la coqueluche. Ann Inst Pasteur (Paris) 20, 731741 (1906).

31 Stainer, D. W. \& Scholte, M. J. A simple chemically defined medium for the production of phase I Bordetella pertussis . J Gen Microbiol 63, 211-220 (1970).

32 Eby, J. C., Gray, M. C., Warfel, J. M., Merkel, T. J. \& Hewlett, E. L. Use of a Toxin Neutralization Assay To Characterize the Serologic Response to Adenylate Cyclase Toxin after Infection with Bordetella pertussis . Clin Vaccine Immunol 24, doi:10.1128/CVI.00370-16 (2017).

33 Nguyen, A. W. et al. A cocktail of humanized anti-pertussis toxin antibodies limits disease in murine and baboon models of whooping cough. Science translational medicine 7, 316ra195-316ra195, doi:10.1126/scitranslmed.aad0966 (2015).

34 Wagner, E. K., Wang, X., Bui, A. \& Maynard, J. A. Synergistic Neutralization of Pertussis Toxin by a Bispecific Antibody In Vitro and In Vivo. Clinical and vaccine immunology : CVI 23, 851-862, doi:10.1128/CVI.00371-16 (2016).

35 Guiso, N., Szatanik, M. \& Rocancourt, M. Protective activity of Bordetella adenylate cyclase-hemolysin against bacterial colonization. Microb Pathog 11, 423-431 (1991).

36 Hormozi, K., Parton, R. \& Coote, J. Toxicity tests on native and recombinant Bordetella pertussis adenylate cyclase toxin preparations. Developments in biological standardization 101, 147-154 (1999).

37 Macdonald-Fyall, J. et al. Adjuvanticity of native and detoxified adenylate cyclase toxin of Bordetella pertussis towards co-administered antigens. Vaccine 22, 4270-4281, doi:10.1016/j.vaccine.2004.04.033 (2004).

38 Orr, B., Douce, G., Baillie, S., Parton, R. \& Coote, J. Adjuvant effects of adenylate cyclase toxin of Bordetella pertussis after intranasal immunisation of mice. Vaccine 25, 64-71, doi:10.1016/j.vaccine.2006.07.019 (2007). 
39 Villarino Romero, R. et al. The Bordetella pertussis type III secretion system tip complex protein Bsp22 is not a protective antigen and fails to elicit serum antibody responses during infection of humans and mice. Infect Immun 81, 27612767, doi:10.1128/IAI.00353-13 (2013).

40 Weiner, Z. P. et al. Circulating lethal toxin decreases the ability of neutrophils to respond to Bacillus anthracis. Cell Microbiol 16, 504-518, doi:10.1111/cmi.12232 (2013).

41 Basler, M., Masin, J., Osicka, R. \& Sebo, P. Pore-forming and enzymatic activities of Bordetella pertussis adenylate cyclase toxin synergize in promoting lysis of monocytes. Infect Immun 74, 2207-2214, doi:10.1128/IAI.74.4.22072214.2006 (2006).

42 Confer, D. L. \& Eaton, J. W. Phagocyte impotence caused by an invasive bacterial adenylate cyclase. Science 217, 948-950 (1982).

43 Hewlett, E. L., Donato, G. M. \& Gray, M. C. Macrophage cytotoxicity produced by adenylate cyclase toxin from Bordetella pertussis : more than just making cyclic AMP! Mol Microbiol 59, 447-459, doi:10.1111/j.1365-2958.2005.04958.x (2006).

44 Guiso, N., Capiau, C., Carletti, G., Poolman, J. \& Hauser, P. Intranasal murine model of Bordetella pertussis infection. I. Prediction of protection in human infants by acellular vaccines. Vaccine 17, 2366-2376, doi:S0264410X99000377 [pii] (1999).

45 Alvarez Hayes, J. et al. Bordetella pertussis iron regulated proteins as potential vaccine components. Vaccine 31, 3543-3548, doi:10.1016/j.vaccine.2013.05.072 (2013).

46 de Gouw, D. et al. The vaccine potential of Bordetella pertussis biofilm-derived membrane proteins. Emerging microbes \& infections 3, e58, doi:10.1038/emi.2014.58 (2014).

47 Belcher, C. E. et al. The transcriptional responses of respiratory epithelial cells to Bordetella pertussis reveal host defensive and pathogen counter-defensive strategies. Proc Natl Acad Sci U S A 97, 13847-13852, doi:10.1073/pnas.230262797 (2000).

48 Zhang, X., Goel, T., Goodfield, L. L., Muse, S. J. \& Harvill, E. T. Decreased leukocyte accumulation and delayed Bordetella pertussis clearance in IL-6-/mice. J Immunol 186, 4895-4904, doi:10.4049/jimmunol.1000594 (2011).

49 Perkins, D. J., Gray, M. C., Hewlett, E. L. \& Vogel, S. N. Bordetella pertussis adenylate cyclase toxin (ACT) induces cyclooxygenase-2 (COX-2) in murine macrophages and is facilitated by ACT interaction with CD11b/CD18 (Mac-1). Mol Microbiol 66, 1003-1015, doi:10.1111/j.1365-2958.2007.05972.x (2007).

50 Andreasen, C., Powell, D. A. \& Carbonetti, N. H. Pertussis toxin stimulates IL-17 production in response to Bordetella pertussis infection in mice. PLoS One 4, e7079, doi:10.1371/journal.pone.0007079 (2009). 
51 Barbic, J., Leef, M. F., Burns, D. L. \& Shahin, R. D. Role of gamma interferon in natural clearance of Bordetella pertussis infection. Infect Immun 65, 4904-4908 (1997).

52 Mahon, B. P., Sheahan, B. J., Griffin, F., Murphy, G. \& Mills, K. H. Atypical disease after Bordetella pertussis respiratory infection of mice with targeted disruptions of interferon-gamma receptor or immunoglobulin mu chain genes. The Journal of experimental medicine 186, 1843-1851 (1997).

53 Barnard, A., Mahon, B. P., Watkins, J., Redhead, K. \& Mills, K. H. G. Th1/Th2 cell dichotomy in acquired immunity to Bordetella pertussis : Variables in the in vivo priming and in vitro cytokine detection techniques affect the classification of T-cell subsets as Th1, Th2 or Th0. Immunology 87, 372-380, doi:DOI 10.1046/j.1365-2567.1996.497560.x (1996).

54 Place, D. E., Muse, S. J., Kirimanjeswara, G. S. \& Harvill, E. T. Caspase-1independent interleukin-1beta is required for clearance of Bordetella pertussis infections and whole-cell vaccine-mediated immunity. PLoS One 9, e107188, doi:10.1371/journal.pone.0107188 (2014).

55 Guermonprez, P. et al. The adenylate cyclase toxin of Bordetella pertussis binds to target cells via the alpha(M)beta(2) integrin (CD11b/CD18). The Journal of experimental medicine 193, 1035-1044 (2001).

56 Andreasen, C. \& Carbonetti, N. H. Role of neutrophils in response to Bordetella pertussis infection in mice. Infect Immun 77, 1182-1188, doi:10.1128/IAI.0115008 (2009).

57 Kirimanjeswara, G. S., Agosto, L. M., Kennett, M. J., Bjornstad, O. N. \& Harvill, E. T. Pertussis toxin inhibits neutrophil recruitment to delay antibody-mediated clearance of Bordetella pertussis . J Clin Invest 115, 3594-3601, doi:10.1172/JCl24609 (2005).

58 Boursaux-Eude, C., Thiberge, S., Carletti, G. \& Guiso, N. Intranasal murine model of Bordetella pertussis infection: II. Sequence variation and protection induced by a tricomponent acellular vaccine. Vaccine 17, 2651-2660 (1999).

59 Khelef, N., Zychlinsky, A. \& Guiso, N. Bordetella pertussis induces apoptosis in macrophages: role of adenylate cyclase-hemolysin. Infect Immun 61, 4064-4071 (1993).

60 Mahon, B. P., Ryan, M. S., Griffin, F. \& Mills, K. H. Interleukin-12 is produced by macrophages in response to live or killed Bordetella pertussis and enhances the efficacy of an acellular pertussis vaccine by promoting induction of Th1 cells. Infect Immun 64, 5295-5301 (1996).

61 Weigand, M. R. et al. The History of Bordetella pertussis Genome Evolution Includes Structural Rearrangement. J Bacteriol 199, doi:10.1128/JB.00806-16 (2017).

62 Martin, S. W. et al. Pertactin-negative Bordetella pertussis strains: evidence for a possible selective advantage. Clin Infect Dis 60, 223-227, doi:10.1093/cid/ciu788 (2015). 
63 Williams, M. M. et al. Bordetella pertussis Strain Lacking Pertactin and Pertussis Toxin. Emerg Infect Dis 22, 319-322, doi:10.3201/eid2202.151332 (2016).

64 Arico, B. \& Rappuoli, R. Bordetella parapertussis and Bordetella bronchiseptica contain transcriptionally silent pertussis toxin genes. J Bacteriol 169, 2847-2853 (1987).

65 Pittet, L. F., Emonet, S., Schrenzel, J., Siegrist, C. A. \& Posfay-Barbe, K. M. Bordetella holmesii: an under-recognised Bordetella species. The Lancet. Infectious diseases 14, 510-519, doi:10.1016/S1473-3099(14)70021-0 (2014).

66 Bart, M. J. et al. Global population structure and evolution of Bordetella pertussis and their relationship with vaccination. $m B i o 5$, e01074, doi:10.1128/mBio.0107414 (2014).

67 Higgs, R., Higgins, S. C., Ross, P. J. \& Mills, K. H. Immunity to the respiratory pathogen Bordetella pertussis . Mucosal Immunol 5, 485-500, doi:10.1038/mi.2012.54 (2012). 


\section{Chapter 4- Intranasal acellular pertussis vaccine provides mucosal immunity and protects mice from Bordetella pertussis}

Dylan T. Boehm ${ }^{a, b}$, M. Allison Wolfa,b, Jesse M. Hall ${ }^{a, b}$, Ting Y. Wong ${ }^{a, b}$, Emel SenKilic ${ }^{a, b}$, M. de la Paz Gutierrez ${ }^{c}$, Catherine Blackwood ${ }^{a, b}$, Shelby D. Bradford ${ }^{a, b}$, Katherine A. Begley ${ }^{a, b}$, William T. Witt ${ }^{a, b}$, Mariette Barbier ${ }^{a, b}$ and F. Heath Damron ${ }^{a, b^{*}}$

aDepartment of Microbiology, Immunology, and Cell Biology, West Virginia University, Morgantown, WV, USA ; 'baccine Development center at West Virginia University Health Sciences Center West Virginia University, Morgantown, WV, USA; Instituto de Biotecnología y Biología Molecular (IBBM)-CCT-CONICET-La Plata, Departamento de Ciencias Biológicas, Facultad de Ciencias Exactas, Universidad Nacional de La Plata, La Plata, Argentina.

Submitted in 2019 to Vaccines - Manuscript under consideration 


\section{Abstract}

Current acellular pertussis vaccines fall short of optimal protection against the human respiratory pathogen Bordetella pertussis resulting in increased incidence of a previously controlled vaccine preventable disease. Natural infection is known to induce a protective mucosal immunity. Therefore, in this study, we aimed to use acellular pertussis vaccines to recapitulate these mucosal immune responses. We utilized a murine immunization and challenge model to characterize the efficacy of intranasal immunization (IN) with DTaP vaccine or DTaP vaccine supplemented with curdlan, a known Th1/Th17 promoting adjuvant. Protection from IN delivered DTaP was compared to protection mediated by intraperitoneal injection of DTaP and whole-cell pertussis vaccines. We tracked fluorescently labeled DTaP after immunization and detected that $\mathrm{DTaP}$ localized preferentially in the lungs while DTaP with curdlan was predominantly in the nasal turbinates. IN immunization with DTaP, with or without curdlan adjuvant, resulted in anti-B. pertussis and anti-pertussis toxin IgG titers at the same level as intraperitoneally administered DTaP. IN immunization was able to protect against $B$. pertussis challenge and we observed decreased pulmonary pro-inflammatory cytokines, macrophage and neutrophil infiltrates in the lung, and bacterial burden in the upper and lower respiratory tract at day 3 post challenge. Furthermore, IN immunization with DTaP triggered mucosal immune responses such as production of $B$. pertussis-specific $\lg A$, and increased IL-17A. Together, the induction of a mucosal immune response and humoral antibody-mediated protection associated with an IN administered DTaP and curdlan adjuvant warrant further exploration as a pertussis vaccine candidate formulation. 


\section{Introduction}

Pertussis is a human upper respiratory disease, primarily caused by the Gram-negative pathogen Bordetella pertussis. The disease is most severe in unvaccinated infants, where it manifests as fits of paroxysmal coughs followed by the classical whoop as air re-enters the respiratory system, leading to the more commonly known name: whooping cough. ${ }^{1}$ Additional complications include vomiting, increased mucus production, and apneic episodes. In severe cases, these symptoms can lead to elevated leukocytosis, pulmonary hypertension, hypoxia, and in some cases death. ${ }^{1-3}$ Furthermore there is speculation that pertussis may enhance development of neurodegenerative diseases later in life. ${ }^{4}$

In the pre-vaccine era, pertussis was responsible for 200,000 deaths annually in the United States. ${ }^{5}$ Widespread use of diphtheria tetanus whole-cell pertussis (DTP; wP) vaccines controlled the incidence of pertussis. ${ }^{6}$ However, adverse side effects associated with WP immunization ${ }^{1}$ led to the development of an acellular pertussis vaccine (DTaP; aP) that was introduced into the schedule in 1996. In the United States the number of pertussis cases has been increasing since the 1990s, despite high vaccine coverage. ${ }^{7}$ Additionally, increasing numbers of cases have been documented in older children, adolescents and adults. ${ }^{8}$ The increase in incidence of pertussis cases suggests that current aP vaccines do not offer complete protection from $B$. pertussis infection. Multiple hypotheses have been proposed explaining the resurgence of pertussis cases including: (1) waning of protective immunity from DTaP/tdap ${ }^{9-11}$, (2) vaccine driven evolution of $B$. pertussis strains ${ }^{12}$, (3) increased carriage and transmission through asymptomatic carriers ${ }^{13}$, and (4) increased surveillance and more accurate diagnoses technology.

Vaccine-induced protection in aP immunized individuals has been associated with a robust antigen-specific IgG response to the components of the aP vaccines. ${ }^{14-17}$ Likewise, WP immunization also resulted in antigen-specific IgG responses; with the addition of a shift to a more diverse $T$ cell response, inducing cell-mediated immunity. ${ }^{18}$ In the murine model, immunization through intramuscular (IM) and intraperitoneal (IP) administration has been well characterized demonstrating a Th1/Th17 response from 
WP immunized mice, and a Th2 with weak Th17 mediated response in aP immunized mice following $B$. pertussis challenge ${ }^{19}$ However, these immunizations fail to induce the mucosal immune responses elicited from natural infection. Recent studies have revealed that protection correlates with tissue resident memory $\mathrm{T}$ ( $\mathrm{T}_{\mathrm{RM}}$ ) cells in the lung and nasal cavity of convalescent mice, that produce interleukin-17 (IL-17) and interferon-gamma (IFN-Y). ${ }^{20} \mathrm{~T}_{\mathrm{RM}}$ cells have been shown to persist in the respiratory tissue and expand upon re-challenge of a convalescent mouse with B. pertussis, as well as decrease bacterial burden upon adoptive transfer to naïve mouse. ${ }^{21}$ The study of this population has renewed interest in the induction of a mucosal immune response capable of decreasing bacterial burden at the site of infection.

The induction of a mucosal immune response to $B$. pertussis is associated with the production of secretory $\lg A$ antibodies ( $\lg A$ ) in the nasal cavity. In humans previously infected with $B$. pertussis, IgA antibodies have been isolated from nasal secretions. ${ }^{22} B$. pertussis-specific IgA antibodies isolated from convalescent patients have been shown to inhibit adherence of $B$. pertussis to respiratory epithelial cells in vitro ${ }^{23}$, suggesting a protective role of IgA antibodies in mucosal immunity. Initial studies using IgA-deficient mice did not show strong support for a critical role in bacterial clearance of the respiratory tract. ${ }^{24}$ However, work with a live-attenuated IN pertussis vaccine (BPZE1) recently demonstrated protective role of slgA antibodies in the respiratory tract. ${ }^{25}$

DTaP vaccine does not contain a strong pro-inflammatory adjuvant such as endotoxin of wP vaccine or BPZE1. We aimed to investigate IN DTaP immunization alone or with an additional pro-inflammatory adjuvant. We formulated vaccines containing the adjuvant curdlan, a 1,3 $\beta$-glucan, derived from Alcaligenes faecalis. This polysaccharide has immunostimulatory properties and forms a "sticky" gel at a neutral $\mathrm{pH}$. Importantly, curdlan has been shown to bind to dendritic cells through the ligand Dectin-1, thereby inducing expression of NF-KB ${ }^{26}$ leading to a Th1/Th17 mediated immune response as well as production of antigen-specific respiratory IgA antibodies and serum IgG antibodies. ${ }^{26-29}$ Our objective was to determine whether IN DTaP immunization would induce a protective immune response in a murine model. We observed that the gel properties of curdlan facilitated DTaP localization in the upper respiratory tract. We 
measured serum and respiratory antibody responses that correlated with protection from bacterial challenge. This study suggests mucosal vaccination with acellular vaccine may be a strategy for decreasing incidence of pertussis.

\section{$\underline{\text { Results }}$}

Acellular pertussis vaccine was retained in the upper and lower respiratory tract when administered by intranasal administration.

Pertussis toxin facilitates infection by disrupting innate immune responses in the airway. 30 Therefore, we hypothesized that neutralization of pertussis toxin at the site of infection is essential for protection against $B$. pertussis. We propose that intranasal immunization would prime a protective mucosal immune response. Furthermore, the gel-like properties of curdlan may have a beneficial role in increasing antigen uptake. To determine if use of curdlan would increase vaccine retention in the respiratory system, we IN vaccinated CD-1 mice with commercially available DTaP (IN-aP), DTaP with curdlan (IN-caP), or PBS (mock vaccinated) and tracked the vaccine up to $48 \mathrm{~h}$ after vaccination (Fig. 1a). Using in vivo animal imaging, we observed fluorescently labeled DTaP particles in the nasal cavity at 6, 12 and $24 \mathrm{~h}$ post-vaccination (Fig. 1b). At $12 \mathrm{~h}$ post-vaccination, we detected significantly higher levels of fluorescence in IN-caP vaccinated mice compared to IN-aP mice (Fig. 1c), suggesting more DTaP particles were retained in the nasal cavity. This method resulted in the quantification of total particles in nasal cavity. To quantify DTaP particles that were bound to innate immune cells we utilized flow cytometry. Single-cell suspensions were prepared from homogenized lung tissue and antigen presenting cells (APCs) bound to DTaP were quantified as live, single cells positive for $\mathrm{CD}_{11 \mathrm{~b}^{+} \mathrm{DTaP}}{ }^{+}$(Fig. 1d). We observed a significant increase in $\mathrm{CD} 11 \mathrm{~b}^{+}$cells that were bound to or contained DTaP particles in $\mathrm{IN}$-aP mice compared to IN-caP (Fig. 1e). Together, these data suggest a higher deposition of DTaP in the lung with IN-aP when compared to IN-caP. Conversely, in the nasal cavity we measured higher levels of DTaP when mice were vaccinated with INcaP, compared to IN-aP. These findings suggest that addition of curdlan to the DTaP vaccine causes retention in the nasal cavity, but without it, the vaccine components are more readily detected in the lung. 
To visualize the deposition of DTaP particles, sections from the lung and nasal cavity were imaged using confocal microscopy. Vaccinated mice were euthanized after $6 \mathrm{~h}$, lung tissue was flash frozen and skulls were embedded in paraffin for sectioning. Sections from the lung and nasal cavity were counterstained with NucBlue and ActinGreen to visualize epithelial tissue and fluorescent DTaP particles (Fig. 2ac). Vaccine particles were quantified by measuring the percentage of total image field emitting DTaP fluorescence. We detected a significant increase of fluorescent particles in the lungs of mice that were vaccinated with IN-aP compared to IN-caP (Fig. 2b). Using microscopy, there was no significant difference in the number of particles detected in the nares comparing IN-aP to IN-caP (Fig. 2d). Interestingly, we observed that DTaP particles from the IN-aP vaccinated mice were localized in the lumen of the nasal passages, while particles from IN-caP vaccinated mice were deposited into the epithelial cells (Fig. 2c). Overall these data suggest curdlan impacts localization of DTaP in the airway.

Intranasal immunization induces production of anti-PT and anti-FHA IgG in serum

To determine vaccine induced protection, we utilized an established vaccine and challenge protocol. ${ }^{31} \mathrm{CD}-1$ mice were immunized IN with either IN-aP or IN-caP. These groups were compared to pertussis immunizations that are known to be protective: DTaP (IP-aP) and whole-cell pertussis (IP-wP) administered intraperitoneally (IP). Additionally, we aimed to determine if the effects of curdlan were dependent by route, thus we included mice immunized with IP delivered DTaP and curdlan (IP-caP). Lastly, we compared the findings to mock vaccinated mice, with IN delivered PBS, or mice IN immunized with curdlan only (IN-curdlan). All mice were immunized with $1 / 12^{\text {th }}$ of the antigen load contained in the human vaccine dose. Mice were vaccinated at day 0 , then received a booster vaccine at day 21 . Mice were challenged on day 35 with $B$. pertussis.

We first sought to determine if an IN delivered DTaP vaccine would induce a systemic immune response. To answer this, we performed enzyme-linked immunosorbent assays (ELISAs) with serum from vaccinated and challenged mice against $B$. pertussis antigens found in the vaccine: pertussis toxin (PT) and filamentous hemagglutinin (FHA). We did 
not detect a significant difference in serum IgG titers between collection timepoints at days 1 and 3 post challenge (pc) (data not shown). Serum anti-PT IgG titers were similar between mice immunized with IP-aP and those immunized through IN administration, as no significant differences were determined between IP-aP, IN-aP or IN-caP (Fig. 3a). Similarly, we observed a robust titer response to the bacterial adhesin FHA in IN vaccinated mice; however, IN-aP serum anti-FHA titer was 7-fold higher than IN-caP (Fig. 3b). We next determined if IN administration of DTaP (with or without curdlan) would lead to an increased Th1 immune response resulting in a higher ratio of IgG2a compared to IgG1 antibodies. Neither route nor adjuvant impacted the ratio of $\lg G 2 a$ to $\lg G 1$.

$\underline{\text { Intranasal immunization induces production of anti-B. pertussis } \lg A \text { in lung }}$

In the murine model, recent data has pointed to the importance of $\lg A$ and a local mucosal immune response in the lung and nasal cavity. ${ }^{32-36}$ We aimed to measure the presence of IgA antibodies in the murine respiratory tissue due to IN immunization. Using ELISAs we measured $B$. pertussis specific IgA titers in homogenized lung tissue supernatant and nasal lavage fluid. Interestingly, we detected a robust IgA response in the lung only when mice were immunized through the IN route (Fig. 4a). Antigenspecific IgA response was not observed in mice immunized with curdlan alone. We observed detectable IgA $B$. pertussis-titer in the nasal lavage fluid from both IN-aP and $\mathrm{IN}$-caP vaccinated groups, although only $\mathrm{IN}-\mathrm{aP}$ resulted in a significant increase compared to baseline levels (Fig. 4b). The presence of B. pertussis binding IgA in the lungs and nasal cavity suggests that IN DTaP is capable of priming a mucosal immune response in the upper and lower murine respiratory systems.

\section{$\underline{\text { Intranasal immunization decreased pulmonary pro-inflammatory environment }}$}

It has been well established that neutralization of PT and inhibition of bacterial adhesins associated with DTaP protection leads to a markedly reduced pro-inflammatory environment at the site of infection when compared to a natural infection. ${ }^{17}$ Conversely, challenge in whole-cell protected animals resulted in a severe pro-inflammatory response, similar to the natural infection of $B$. pertussis. ${ }^{37}$ We hypothesized that the immunostimulatory properties of curdlan would induce a more pro-inflammatory 
response following challenge with $B$. pertussis compared to IP-aP. Cytokine concentrations were determined from supernatant of lung homogenate at day $3 \mathrm{pc}$. The pro-inflammatory interleukin-6 (IL-6) has been shown to be increased following $B$. pertussis infection of a naïve mouse, while DTaP induced protection drastically decreases IL-6 production. ${ }^{31,38}$ Our results demonstrate a significant reduction of IL-6 in the lungs of either $\mathrm{IN}-\mathrm{aP}$ or $\mathrm{IN}-\mathrm{caP}$ immunized mice when compared to mock vaccinated or IN-curdlan control mice, and are comparable to levels observed in IP immunized groups (Fig. 5a). We measured a similar reduction in the Th1 associated cytokine IFN- $\gamma$ in the IN administered groups, IN-aP and IN-caP; although, these levels were higher in IN-caP immunized mice (Fig. 5b). We only observed a significant increase in the Th2 associated interleukin-5 in IN-wP immunized mice compared to mock vaccinated mice (Fig. 5c).

IN administration of curdlan, and moreover, vaccine administration through the IN route regardless of adjuvant has been shown to induce an increased IL-17 response. ${ }^{27,28}$ Thus, we hypothesized that we would observe increased levels of IL-17 in mice immunized with IN-caP compared to IN-aP or IP-aP. We quantified IL-17A in lung supernatant and observed significant increases of IL-17A with the addition of curdlan in IP-caP and IN-caP immunized mice supporting our hypothesis (Fig. 5d). Furthermore, we observed that IN administration induced a significant increase in IL-17A compared to IP immunization. This IL-17A response was lower than the robust IL-17A induced by IPwP (Fig. 5d).

Natural infection with $B$. pertussis is known to cause severe leukocytosis in the murine model ${ }^{39}$, which can be measured by elevated neutrophils in the peripheral blood. Following $B$. pertussis challenge, we observed that all vaccinated groups ameliorated symptoms of leukocytosis; however, only the administration of DTaP either by IP or IN administration significantly reduced $C D 11 b^{+} G r-1^{\text {hi }}$ neutrophils in the peripheral blood (Fig. 6a). In the lungs, neutrophils were decreased in IN-aP, IN-caP, and IP-aP immunized groups compared to mock vaccinated mice. We observed a similar finding in pulmonary macrophages, although we only determined a significant decrease in mice immunized with IN-caP compared to mock vaccinated mice (Fig. 6c). Taken together 
these data suggest that immunization with DTaP through the IN route reduces the proinflammatory environment of the murine lung during $B$. pertussis challenge.

Intranasal immunization reduced respiratory $B$. pertussis bacterial burden.

Lastly, we examined the clearance of $B$. pertussis from the respiratory tract following IN immunization. At days 1 and $3 \mathrm{pc}$, viable bacterial burden was quantified by counting of CFU in the lung, trachea, and nasal lavage fluid. We observed a significant reduction in viable bacteria recovered from the lung in all immunized groups by day $3 \mathrm{pc}$; however, these changes were not observed at day 1 pc (Fig. 7ab). IN-aP and IN-caP immunized mice bacterial burdens were reduced by $99.4 \%$ and $99.7 \%$, respectively, compared to mock vaccinated mice. This reduction in viable bacterial burden was similar to that of mice immunized by IP-aP, an immunization that is known to be effective (Fig. 7b). Importantly, this reduction in bacterial burden was not observed following immunization with curdlan alone, further suggesting an antigen-specific response. Similar trends were observed in the trachea homogenate (Fig. 7cd), and nasal lavage fluid (Fig. 7ef), as all immunized groups regardless of IP or IN delivery cleared $B$. pertussis to the limits of detection. In summary, we observed similar clearance of $B$. pertussis from the respiratory tract of mice immunized IN compared to mice immunized with vaccines known to be protection delivered by the IP route.

\section{Discussion}

The natural immune response to $B$. pertussis is initiated by the colonization of the respiratory tract, which leads to a cascade of inflammatory cytokines such as; IL-6, IFN$\gamma$, TNF- $\alpha$, and IL-1 $\beta$ leading to increased inflammatory infiltrate consisting of: dendritic cells, macrophages, neutrophils, and lymphocytes. ${ }^{31,40}$ Numerous studies suggest the infection is cleared through opsonization of bacteria by the humoral immune response, combined with a Th1/Th17 cell-mediated response. ${ }^{15,19,41,42}$ Additionally, factors associated with a local immune responses have been observed in convalescent mice following challenge with $B$. pertussis, such as antigen-specific IgA titers and TRM cells, which were shown to decrease bacterial burden when adoptively transferred to naïve mice. ${ }^{21}$ 
The induction of a protective immune response also has been observed through mucosal immunizations against $B$. pertussis. Oral vaccination with heat-killed $B$. pertussis in newborn infants resulted in the production of $B$. pertussis specific serum and mucosal antibodies; although, this protection was not long lived. ${ }^{43}$ In the mouse model, a live-attenuated IN pertussis vaccine, BPZE1, has shown protection up to one year after immunization ${ }^{44}$, and is in clinical trials. ${ }^{45}$ Furthermore, BPZE1 vaccine produced antigen-specific IgA antibodies in the baboon model, indicative of elicitation of mucosal immune response. ${ }^{46}$ The mucosal immune response was further explored in a murine model where BPZE1 induced a protective secretory IgA immune response, and the generation of long lasting IL-17 secreting $T_{R M}$ cells in the respiratory system. ${ }^{25}$ Similarly, this population of antigen-specific memory cells was found to be expanded by an acellular pertussis vaccine (LP-GMP) consisting of a TLR2 agonist and intracellular receptor stimulator of interferon genes (STING) agonist, which correlated with the IL-17 secreting $T_{R M}$ population. ${ }^{47}$ Promisingly, generation of the IL-17 secreting $T_{R M}$ cell population was observed following whole-cell immunization through IP administration; however, Tdap administration through the same route did not generate this population. ${ }^{20}$ In our study, we demonstrate that by altering the administration route of DTaP immunization from IP to IN delivery, the vaccine still induced a protective immune response. Using fluorescent labeled DTaP particles we visualized that IN-aP was readily detectable (Fig. 1). In vivo imaging analysis demonstrated significantly more labeled IN-caP present in the nasal cavity of mice. We detected a higher proportion of IN-caP particles compared to IN-aP particles that embedded in the epithelium of the nasal turbinates (Fig. 2). These particles had a smaller diameter, which may suggest that when the curdlan adjuvant was included DTaP particles were more readily phagocytosed by APCs compared to IN-aP particles. Further work examining the lymph nodes responsible for antigen priming would be beneficial to determine the role of nasal associated lymphoid tissue compared to vaccination of the lung, and trafficking to mediastinal lymph nodes. The location of antigen priming following IN immunization may play a critical role in the optimization of future human IN vaccines. Determining if antigen uptake in the nasal cavity is essential to induce a protective immune response would play a key role in the development of a human IN pertussis vaccines. 
We determined that while IN-caP vaccinated mice had significantly lower detection of $\mathrm{DTaP}$ in the lungs compared to IN-aP immunized mice that the bacterial clearance was similar between the two groups (Fig. 7), suggesting that antigen priming still occurred. This was further demonstrated through seroconversion as robust antigen-specific serum IgG antibody titers were detected from the two IN immunized groups (Fig. 3). The IgG subclass data (Fig. 3) suggest that immunization with IN-aP most likely induces a Th2 response. Other studies with BPZE1 or LP-GMP adjuvants have observed shifts to Th1 response using IN route. ${ }^{20,25}$

A key finding of our current study was the production of antigen-specific $\lg A$ in the respiratory system only in mice that were administered DTaP through IN administration: IN-aP and IN-caP (Fig. 4). Another difference between the IN-aP and IN-caP groups was the level of IL-17A present in the lung homogenate (Fig. 5). We found that inclusion of curdlan in DTaP resulted in higher levels of IL-17A whether administered through IN or IP route although IL-17A was highest in IN-caP. These findings correlate with results from others suggesting a role of curdlan and IN administration in IL-17 production. ${ }^{27,28}$ Together these data demonstrate induction of mucosal immune response specific to $B$. pertussis in the respiratory system.

In our model, addition of curdlan to DTaP increased IL-17 responses but did not improve clearance (Fig. 7). A caveat of the current work is role of aluminum hydroxide was not addressed. All IN immunizations consisted of DTaP, which contains alum, observations from IN-caP may be affected by the presence of alum. Understanding the role of aluminum hydroxide, along with other potential adjuvants in the mucosal immune response needs to be further studied. Our work does not address long-term protection mediated by $\mathrm{IN}$ administration of DTaP or the inclusion of curdlan adjuvant. Future studies will focus on the longevity of IN immunity.

In conclusion, we observed that by altering the route of administration from IP to IN a protective response was reproduced, resulting in clearance of the pathogen to limits of detection. Furthermore, we determined that IN delivery of DTaP was sufficient to prime a local mucosal immune response at site of infection. The inclusion of the adjuvant curdlan in DTaP immunization did increase IL-17 production in the lung; however, we 
did not observe a shift in the type of immune response compared to DTaP alone. In humans, DTaP and tdap do not provide perfect protection against pertussis; however, there is no clear path forward to the next generation vaccine. It is clear that developing a new vaccine will take considerable efforts. Here in our study we observed a surprising result, that IN DTaP vaccine could protect mice against $B$. pertussis. These data cause us to wonder if a change in the route of DTaP immunization would be a promising booster vaccine strategy.

\section{Materials and Methods}

Composition and administration of vaccines used in study

The vaccines administered in the study were prepared no longer than $1 \mathrm{~h}$ before administration. INFANRIX (GSK) human vaccine (DTaP), and the National Institute for Biological Standards and Control WHO whole-cell pertussis vaccine (NIBSC code 94/532) were used in the study. Vaccines were diluted with PBS to $1 / 12^{\text {th }}$ of the human dose (based on total antigen content). Curdlan (Invivogen, t|rl-curd) was prepared by dissolving $50 \mathrm{mg}$ in $2.5 \mathrm{ml}$ sterile purified water. Curdlan preparation was brought into solution by adding $100 \mu \mathrm{l} 1 \mathrm{~N} \mathrm{NaOH}$ and vortexing. The curdlan suspension $(20 \mathrm{mg} / \mathrm{ml})$ was then sonicated for 10 mins and placed in $37^{\circ} \mathrm{C}$ water bath until administration. Curdlan adjuvant was administered at $200 \mu \mathrm{g}$ per mouse. These experiments were conducted in accordance with the National Institutes of Health Guide for the care and use of laboratory animals. The protocols used were approved by West Virginia University Institutional Animal Care and Use Committees (WVU-ACUC protocol 1602000797).

\section{Vaccination of mice for vaccine particle tracking}

CD-1 (outbred; strain code 022) mice aged four weeks were obtained from Charles River Laboratories. At five weeks mice were anesthetized with $77 \mathrm{mg} / \mathrm{kg}$ ketamine and $7.7 \mathrm{mg} / \mathrm{kg}$ xylazine. Mice were administered $50 \mu \mathrm{l}$ of vaccine or control, with $25 \mu \mathrm{l}$ into each nostril (IN). 


\section{Tracking of DTaP in respiratory system}

DTaP vaccine particles were labeled using the Alexa Fluor 660 Protein Labeling Kit (Molecular Probes). Briefly, $0.5 \mathrm{ml}$ of DTaP vaccine was added to $50 \mu \mathrm{l}$ of $1 \mathrm{M}$ sodium bicarbonate, then added to Alexa Fluor 660 dye stock. The mixture was incubated for 1 $\mathrm{h}$ at room temperature with agitation. The solution was concentrated by dialysis in phosphate-buffered saline overnight to remove unlabeled dye. Labeled vaccine was used to immunize mice. At $0,6,12$ and $24 \mathrm{~h}$ post vaccination fluorescent signal was measured using an IVIS Spectrum (Xenogen). Mice were anesthetized using 3\% isoflurane, mixed with oxygen prior to and throughout imaging. The following parameters were used: 1) A binning setting of 4 was kept constant for all images, 2) each image was quantified using the automatic image setting, 3) fluorescence photons were measured using total radiant efficiency of a common region of interest placed on the nasal cavity, and 4) measurements were normalized using Living Image (Xenogen ver. 2.5). Use of this quantification method accounted for variations in exposure between images.

At $6,12,24$, and $48 \mathrm{~h}$ post vaccination, animals were euthanized to quantify DTaP in lungs by flow cytometry analysis. Lungs were removed, and homogenized using gentleMACS $C$ tubes (Miltenyi Biotec) with enzymatic lung dissociation kit (Miltenyi Biotec, 130-095-927). All samples were blocked using Fc Block (BD), then labelled with Alexa Fluor 700 - conjugated CD11b (Biolegend, 101222), DTaP particles were detected with Cy5 channel. Following a $1 \mathrm{~h}$ dark incubation labeled samples were washed, then fixed using $0.4 \% \mathrm{w} / \mathrm{v}$ paraformaldehyde overnight. Samples were resuspended in PBS and analyzed on a LSR Fortessa flow cytometer (BD). DTaP containing myeloid cells were classified as $C D 11 b^{+} D_{T a P}^{+}$single, live cells.

\section{Detection of DTaP particles in lung and nasal cavity}

Detection of DTaP particles in the lung and nasal cavity were confirmed using confocal imaging. Mock vaccinated and challenged mice were euthanized at $6 \mathrm{~h}$ post vaccination. Prior to homogenization, the post-caval lobe of the mouse lung was 
removed. The post-caval lobe was flash frozen in OCT medium (Tissue Plus, Fisher Healthcare), using liquid nitrogen. The samples were stored at $-80^{\circ} \mathrm{C}$ until sectioning. Sectioned samples $(6 \mu \mathrm{m})$ were fixed in acetone, then stained with ActinGreen Ready Probes (Invitrogen) and NucBlue Ready Probes (Invitrogen), using manufacturer protocols.

Skulls were removed from mouse, and the lower jawbone discarded. The skulls were fixed in formalin for $12 \mathrm{~h}$ at $4^{\circ} \mathrm{C}$, then de-calcified at room temperature for $24 \mathrm{~h}$, before samples were embedded in paraffin. Sectioned samples were de-paraffinized and rehydrated using xylene, and washes with decreasing ethanol concentrations (100 to $70 \%$ ). An antigen retrieval step was performed using citrate buffer, where samples were heated to $98^{\circ} \mathrm{C}$ for 20 mins. Samples were then stained as mentioned above. Samples were analyzed for DTaP particles in tissue and airway mucus using a Nikon A1R confocal microscope. DTaP particles were quantified using ImageJ (version 1.52a). Briefly, the threshold tool was used to select only the fluorescent particles above background levels. Then, the threshold adjusted area was quantified using the analyze particles tool. Thus, the data is represented as the percentage of fluorescent particles per area of the total image field. Three image fields per sample were quantified and averaged per mouse.

\section{B. pertussis strains and growth conditions}

B. pertussis strain UT25Sm1 $1^{48}$ was used for murine challenge in all experiments. UT25Sm1 was cultured as described previously. ${ }^{31}$ Briefly, UT25Sm1 was cultured on Bordet Gengou agar plus 15\% defibrinated sheep's blood (Remel) with streptomycin $100 \mathrm{mg} / \mathrm{ml}$. B. pertussis was incubated at $36^{\circ} \mathrm{C}$ for $48 \mathrm{~h}$, then transferred to modified Stainer-Scholte liquid medium ${ }^{49}$, without the cyclodextrin, heptakis. Liquid cultures were incubated for $24 \mathrm{~h}$ at $36^{\circ} \mathrm{C}$, with shaking until reaching an $\mathrm{OD}_{600}$ of $\sim 0.6$, at which time cultures were diluted for challenge dose. 


\section{Vaccination and B. pertussis challenge}

IN immunized mice received $50 \mu \mathrm{l}$ of vaccine as described above. IP immunized mice received $200 \mu \mathrm{l}$ of vaccine injected into the peritoneal cavity. IN and IP immunized mice received the same antigen dose of $1 / 12^{\text {th }}$. Mice received a boost of the vaccines with the same concentrations twenty-one days after initial immunization. At thirty-five days post initial vaccination, mice were challenged with $2 \times 10^{7} \mathrm{CFU} B$. pertussis administered in $20 \mu \mathrm{l}$ through nostrils. At days 1 and $3 \mathrm{pc}$ mice were euthanized, blood and respiratory tissue were isolated as previously described. ${ }^{31}$

\section{Serological analysis of $B$. pertussis specific antibodies}

Serological responses specific to $B$. pertussis antigens were quantified by ELISA. Highbinding microtiter plates were coated with PT (50ng/well)(LIST Biologicals) and FHA (50ng/well)(Enzo Life Sciences), as described in Boehm et al. ${ }^{31}$ Serological responses against UT25Sm1 were cultured to an $\mathrm{OD}_{600}$ of 0.24 and microtiter plates coated with $50 \mu \mathrm{l}$ of bacteria per well. Bound antibodies were detected using goat anti-mouse IgG, IgA, IgG2a, and IgG1 antibody conjugated to alkaline phosphatase (Southern Biotech). Positive antibody titers were determined using a baseline set at two times the average of blanks.

\section{Quantification of pulmonary and blood APCs}

To determine cell types infiltrating the lung and leukocytes present in peripheral blood, single cell suspensions from the tissues mentioned above were prepared, as described previously. ${ }^{31}$ Briefly, lung tissue was homogenized by Dounce homogenizers, filtered with a $100 \mu \mathrm{m}$ filter, and red blood cells were lysed for $2 \mathrm{~min}$ at $37^{\circ} \mathrm{C}$ (Pharmlyse). Cell populations were determined using antibodies to cell surface markers: PE-conjugated GR-1 (BD, 553128), Alexa Fluor 700-conjugated CD11b (Biolegend, 101222), PerCP/Cy5.5-conjugated F4/80 (Biolegend, 123128). Neutrophils and macrophages were classified as $C D 11 b^{+} G r-1^{\text {hi }}$ single, live cells or $C D 11 b^{+} F 4 / 80^{+}$single, live cells, respectively. 


\section{Lung homogenate cytokine analysis}

To quantify inflammatory cytokines at the site of infection, lung homogenate supernatant was prepared and stored at $-80^{\circ} \mathrm{C}$, as described in prior work. ${ }^{31}$ Quantitative analysis of cytokines was performed using Meso Scale Discovery cytokine kits: V-PLEX proinflammatory panel (K15048D) and IL-17A V-PLEX (K152RFD), per the manufacturer's instructions.

\section{Statistical Analysis}

Experiments in the study were performed with 3 to 8 biological replicates. Data were analyzed using GraphPad Prism 7. Comparisons between groups were performed using one-way analysis of variance (ANOVA) with Dunnett's and Tukey's post hoc tests. Comparisons between groups with or without curdlan were analyzed by two-tailed unpaired t-test, when applicable multiple T-tests with Holm-Sidak post hoc test were applied to curdlan inclusion groups.

\section{Data Availability}

Data for all figures are available upon reasonable request to the corresponding author.

\section{Funding Information}

D.T.B. was supported by a West Virginia Space Grant Consortium Graduate Student Fellowship and a Jennifer Gossling Fellowship. F.H.D. and the Vaccine Development center at WVU-HSC were supported by a Research Challenge Grant No. HEPC.dsr.18.6 from the Division of Science and Research, WV Higher Education Policy Commission. F.H.D. and this work were also supported by NIH 1 R01 Al137155-01A1. Imaging experiments and analysis were performed in the West Virginia University Animal Models \& Imaging Facility, which was supported by the WVU Cancer Institute and NIH grants P20 RR016440, P30 RR032138/GM103488 and U54 GM104942. Imaging experiments and image analysis were performed in the West Virginia University 
Microscope Imaging Facility, which has been supported by the WVU Cancer Institute and NIH grants P20RR016440, P30RR032138/P30GM103488, U54GM104942 and P20GM103434. Flow Cytometry experiments were performed in the West Virginia University Flow Cytometry \& Single Cell Core Facility, which was supported by the National Institutes of Health equipment grant number S10OD016165 and the Institutional Development Awards (IDeA) from the National Institute of General Medical Sciences of the National Institutes of Health under grant numbers P30GM103488 (Cancer CoBRE) and P20GM103434 (INBRE).

\section{Acknowledgements}

We would like to thank the following directors of West Virginia University Core facilities for equipment instruction and advice on developing protocols: Kathleen Brundage (Flow Cytometry Core Facility), Karen Martin and Amanda Ammer (Microscope Imaging Facility), James Coad (Pathology/Histology Core Facility), and Sarah McLaughlin (Animal Models and Imaging Facility). We thank members of F. Heath Damron's and Mariette Barbier's labs for their assistance performing experiments, and review of the manuscript.

\section{Author Contributions}

D.T.B., F.H.D., M.B. designed experiments, performed experiments and composed manuscript. M.A.W., J.M.H., T.Y.W., E.S.K., M.P.G., C.B.B., and W.T.W. performed experiments and reviewed manuscript. S.D.B., and K.A.B. were undergraduate researchers who completed experiments and reviewed the manuscript.

\section{Competing Interests}

The authors declare that there are no competing interests. 


\section{Figures}

Figure 1: Localization of acellular pertussis vaccine in the upper and lower respiratory system after IN vaccination.

a

\begin{tabular}{lllll} 
Time (h): 0 & 6 & 12 & 24 & 48 \\
\hline
\end{tabular}

DTaP Only: IN-aP

DTaP + Curdlan: IN-caP

b

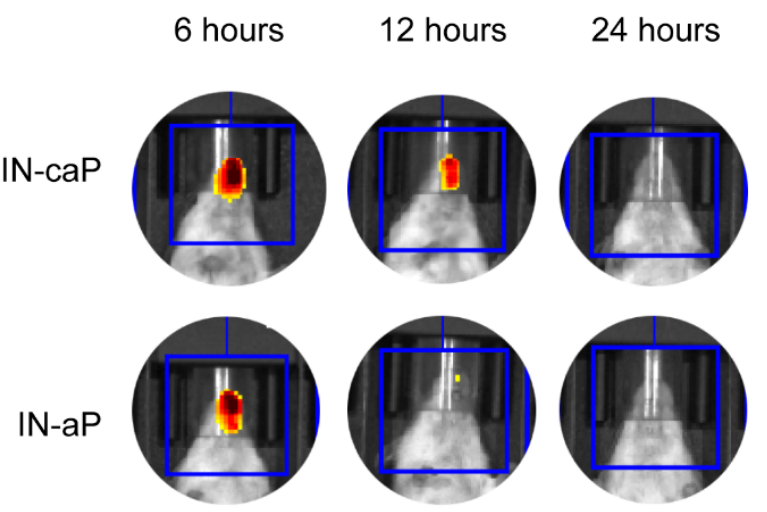

d

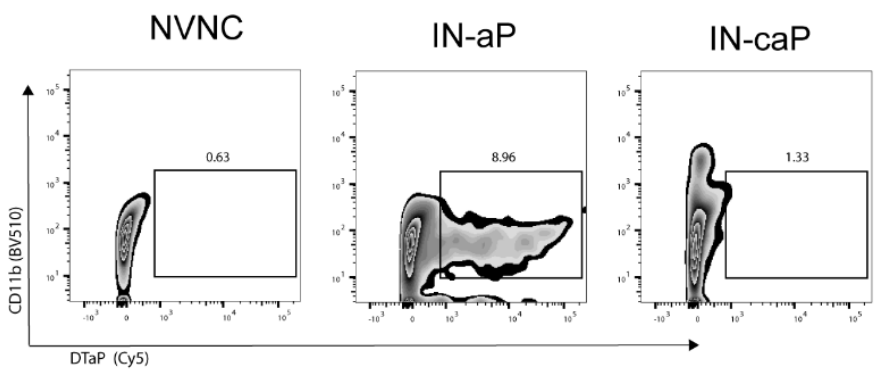

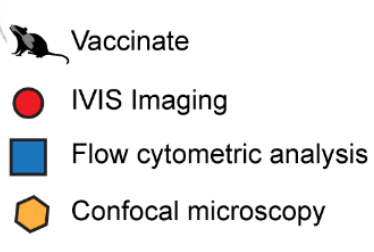

Vaccinate

IVIS Imaging

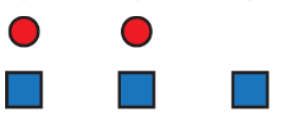

.

0

C

\section{IVIS Floursecence}

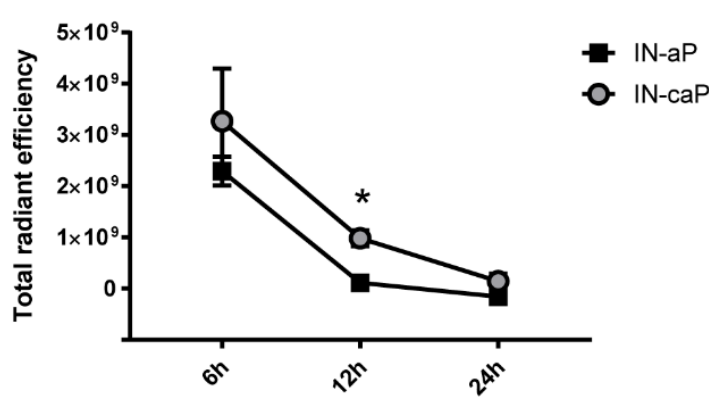

DTaP Accumulation in Lung

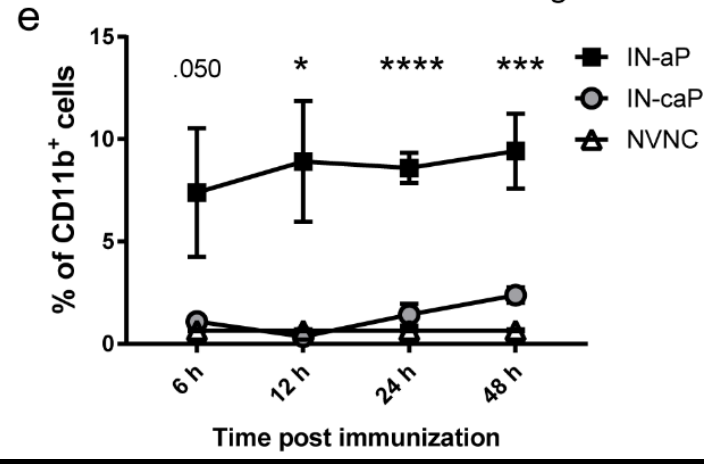

Figure 1: Localization of acellular pertussis vaccine in the upper and lower respiratory system after IN vaccination. (a) Schematic of vaccine tracking protocol. CD-1 mice were intranasally vaccinated with either fluorescent DTaP alone (IN-aP) or fluorescent DTaP with curdlan (IN-caP). Vaccine particle deposition in the lungs and nasal cavity was measured at $0,6,12,24$, and $48 \mathrm{~h}$ after immunization. (b) Representative images of nasal cavity fluorescence at 6,12 , and $24 \mathrm{~h}$. The region of interest used for fluorescence quantification is shown in blue. (c) Fluorescence measurements normalized to PBS control at 6,12 , and $24 \mathrm{~h}$. Results shown as mean \pm 
SEM of total radiant efficiency, ${ }^{\star} P<0.05$. $P$ values were determined by multiple T-tests with Holm-Sidak post hoc test between IN-aP and IN-caP vaccinated mice. (d) Representative plots showing live, single cells that are $C D 11 b^{+} D T a P^{+}$. (e) Flow cytometric analysis of $\mathrm{CD} 11 \mathrm{~b}^{+}$cells from the lung that contain or are bound to DTaP particles at $6,12,24$, and $48 \mathrm{~h}$ post immunization. Results shown as mean $\pm \mathrm{SEM},{ }^{*} P<$ $0.05,{ }^{\star \star \star} P<0.001,{ }^{\star \star \star \star} P<0.0001$. $P$ values were determined by one-way ANOVA with Dunnett's post hoc test comparing IN-aP immunized mice to control mock vaccinated mice. 
Figure 2: Acellular pertussis vaccine particle localization altered by curdlan adjuvant.
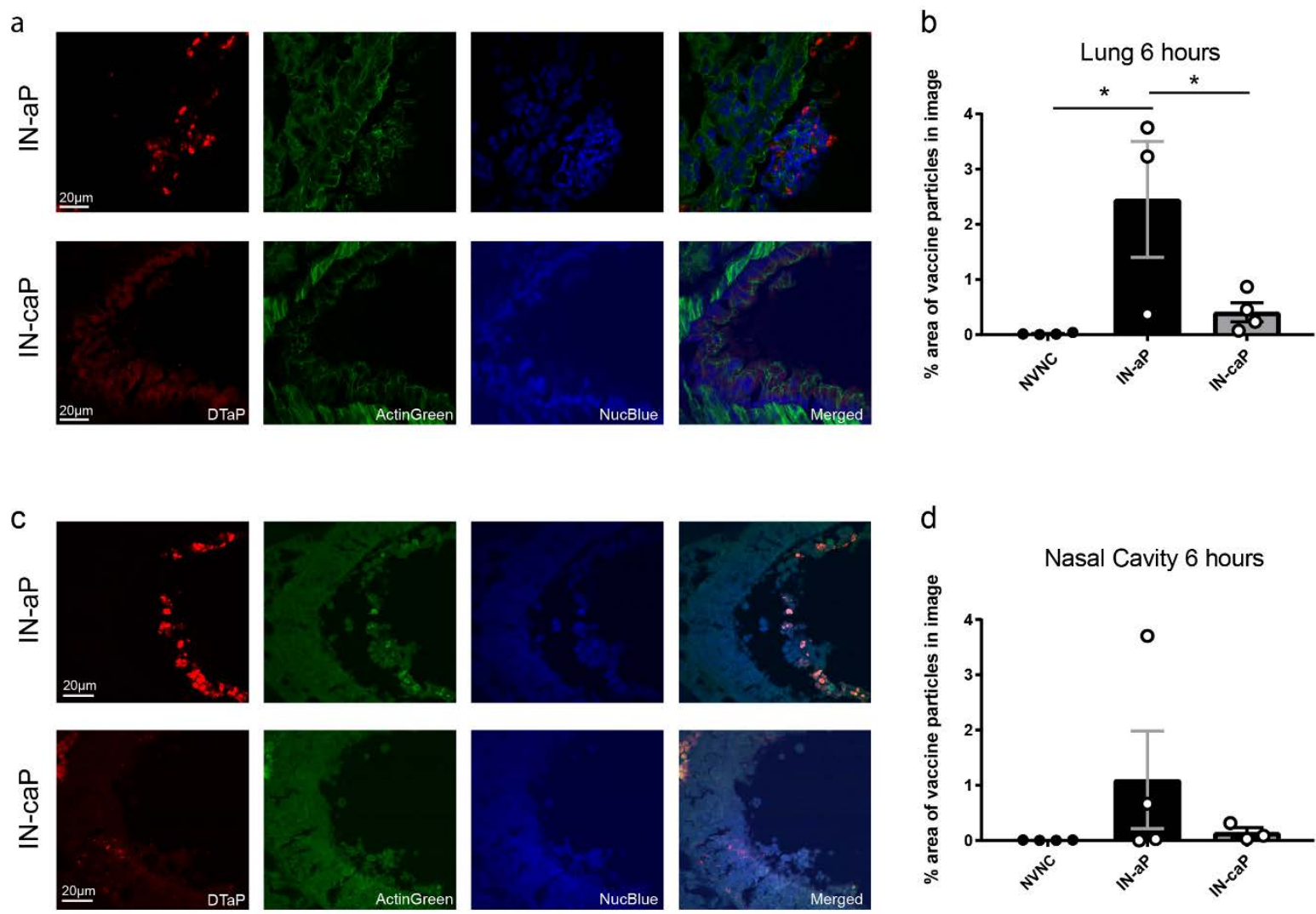

Figure 2: Acellular pertussis vaccine particle localization altered by curdlan adjuvant. (a) Representative images of flash frozen lung sections $6 \mathrm{~h}$ after immunization with IN-aP or IN-caP. Fluorescent particles were detected using a 660 laser, samples were counter-stained with NucBlue (blue) and ActinGreen (green). (b) Fluorescent DTaP particles were quantified by determining the percentage area of particles per field of view. ( $n=3-4$, with averages of 3 images per lung). Results are shown as mean \pm SEM, ${ }^{*} P<0.05$. (c) Representative images of paraffin embedded nasal cavity sections $6 \mathrm{~h}$ after immunization with IN-aP or IN-caP. (d) Fluorescent DTaP particles quantified by determining the percentage area of particles per field of view. ( $n=3-4$, with averages of 3 images per lung). $P$ values were determined by one-way ANOVA with Tukey's post hoc test. 
Figure 3: Intranasal immunization induces production of anti-PT and anti-FHA IgG in serum.
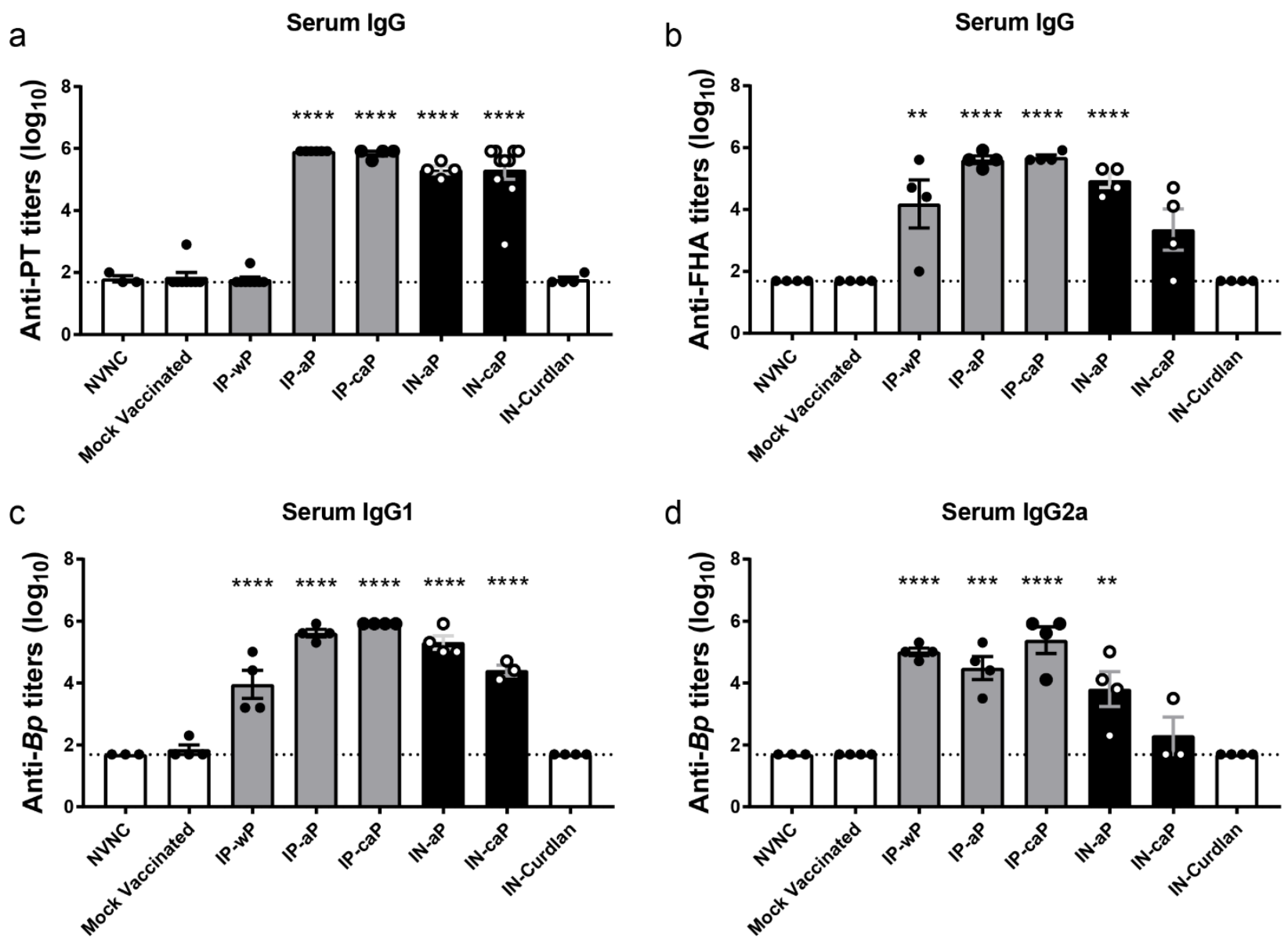

Figure 3: Intranasal immunization induces production of anti-PT and anti-FHA IgG in serum. ELISAs were used to compare serological responses from mice immunized through IN or IP routes to mock vaccinated mice. Total IgG serum antibody titers from immunized and challenged mice were quantified against (a) PT and (b) FHA at day 3 pc. Serum (c) IgG1 and (d) IgG2 antibody titers against $B$. pertussis were compared to mock vaccinated mice at day 3 . Results shown as mean \pm SEM, ${ }^{\star \star} P<0.01,{ }^{\star \star *} P<$ $0.001,{ }^{* \star \star \star} P<0.0001$. $P$ values were determined by one-way ANOVA with Dunnett's post hoc test compared to mock vaccinated mice. 
Figure 4: Intranasal immunization induces production of anti-B. pertussis IgA in respiratory system.

a

\section{Lung IgA}
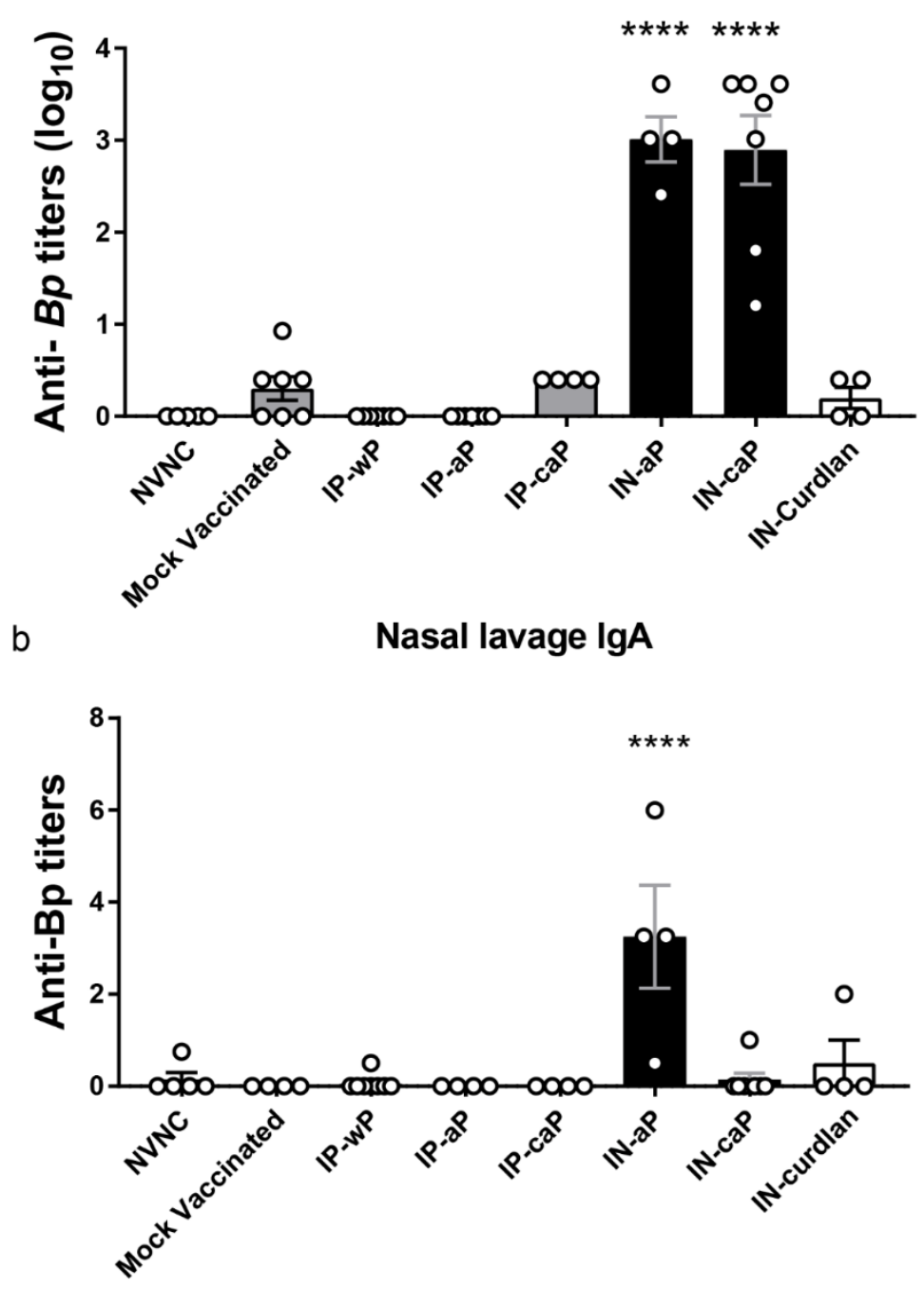

Figure 4: Intranasal immunization induces production of anti-B. pertussis $\lg A$ in respiratory system. ELISAs were performed using (a) lung homogenate supernatant and (b) nasal lavage fluid from vaccinated and challenged mice at day 3 pc. IgA titers were determined against whole-cell $B$. pertussis. Results are shown as averages of two independent experiments, represented on a $\log 10$ scale for lung and linear scale of nasal lavage with mean \pm SEM $(n=4-8)$. ${ }^{\star \star \star \star} P<0.0001$. $P$ values were determined by one-way ANOVA with Dunnett's post hoc test compared to mock vaccinated mice. 
Figure 5: Intranasal immunization decreased pulmonary pro-inflammatory cytokines during challenge.
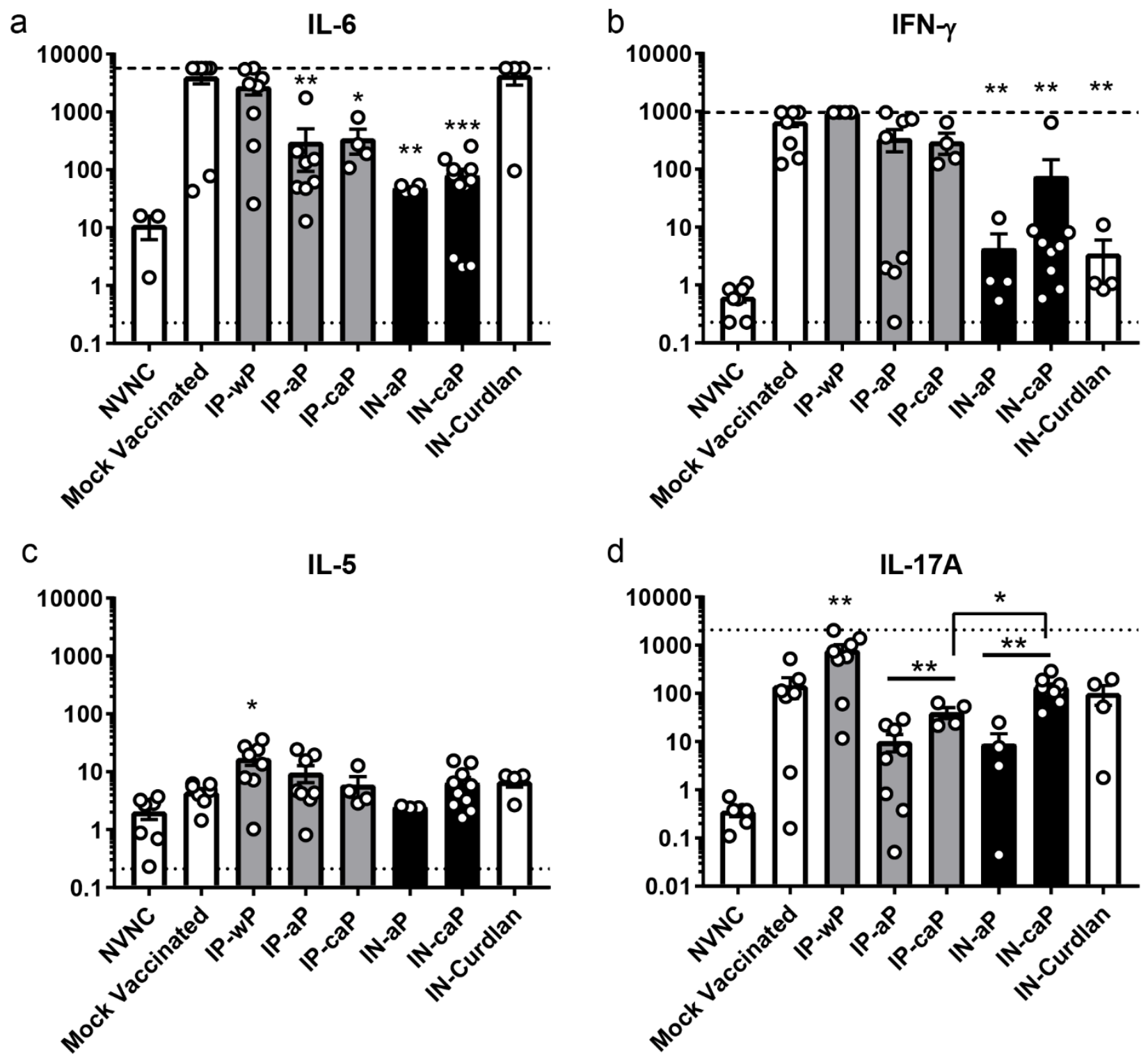

Figure 5: Intranasal immunization decreased pulmonary pro-inflammatory cytokines during challenge. Analysis of cytokines from supernatant of lung homogenate at day 3 pc. Cytokines (a) IL-6, (b) IFN-y, (c) IL-5, (d) IL-17A were quantified by electrochemiluminescence assay. Results shown as mean $\pm \mathrm{SEM}$, ${ }^{\star} P<$ $0.05,{ }^{\star \star} P<0.01,{ }^{\star \star \star} P<0.001,{ }^{\star \star \star \star} P<0.0001$. $P$ values were determined by one-way ANOVA with Dunnett's post hoc test compared to mock vaccinated mice. Bars connecting groups indicate values determined by two-tailed un-paired $t$-test. Upper and lower limits of detection shown as dash or dotted lines, respectively if data points reached these limits. 
Figure 6: Intranasal immunization reduced neutrophil and macrophage accumulation in the lung and circulating neutrophils after $B$. pertussis challenge.

a

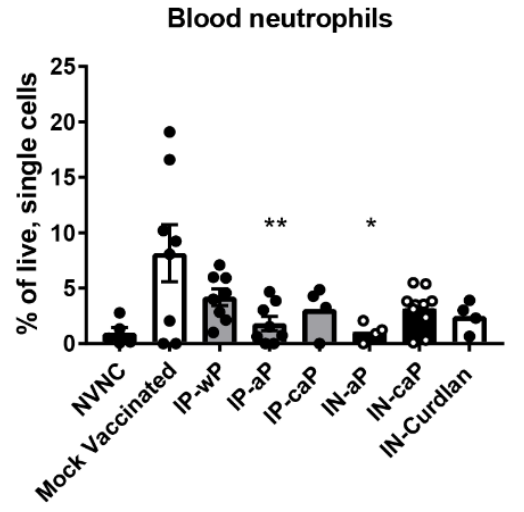

$\mathrm{b}$

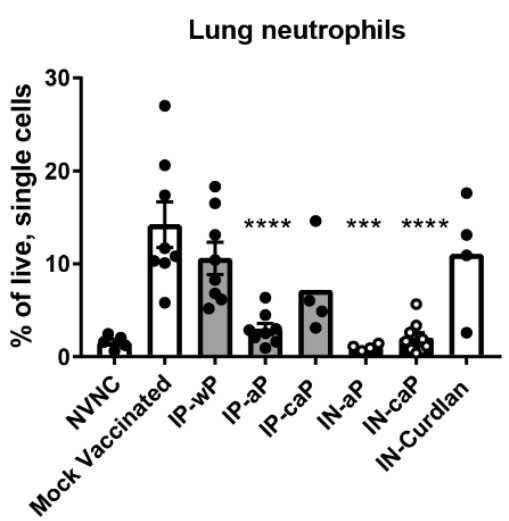

c

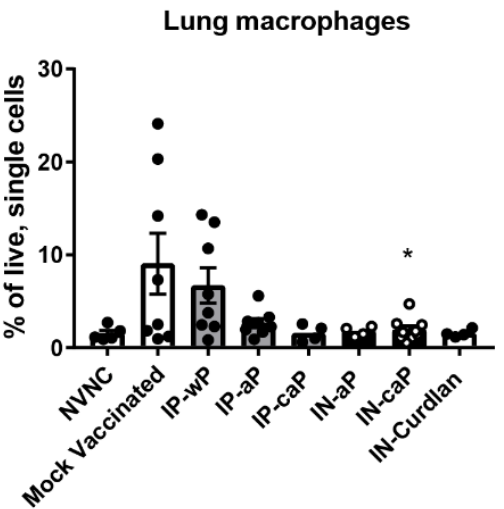

Figure 6: Intranasal immunization reduced neutrophil and macrophage accumulation in the lung and circulating neutrophils after $B$. pertussis challenge. (a) The percentage of live, CD11 $b^{+} G r-1^{\text {hi }}$ neutrophils from a single cell suspension of the peripheral blood. Percentages of (b) $\mathrm{CD} 11 \mathrm{~b}^{+} \mathrm{Gr}-1^{\mathrm{hi}}$ neutrophils and (c) $\mathrm{CD} 11 \mathrm{~b}+\mathrm{F} 4 / 80+$ macrophages in single cell lung homogenates. Results shown as means $\pm \mathrm{SEM},{ }^{\star} P<0.05{ }^{\star \star} P<0.01$, ${ }^{\star \star \star} P<0.001$, ${ }^{\star \star \star \star *} P<0.0001$. $P$ values were determined by one-way ANOVA with Dunnett's post hoc test compared to mock vaccinated mice. 
Figure 7: Intranasal immunization reduced respiratory B. pertussis bacterial burden
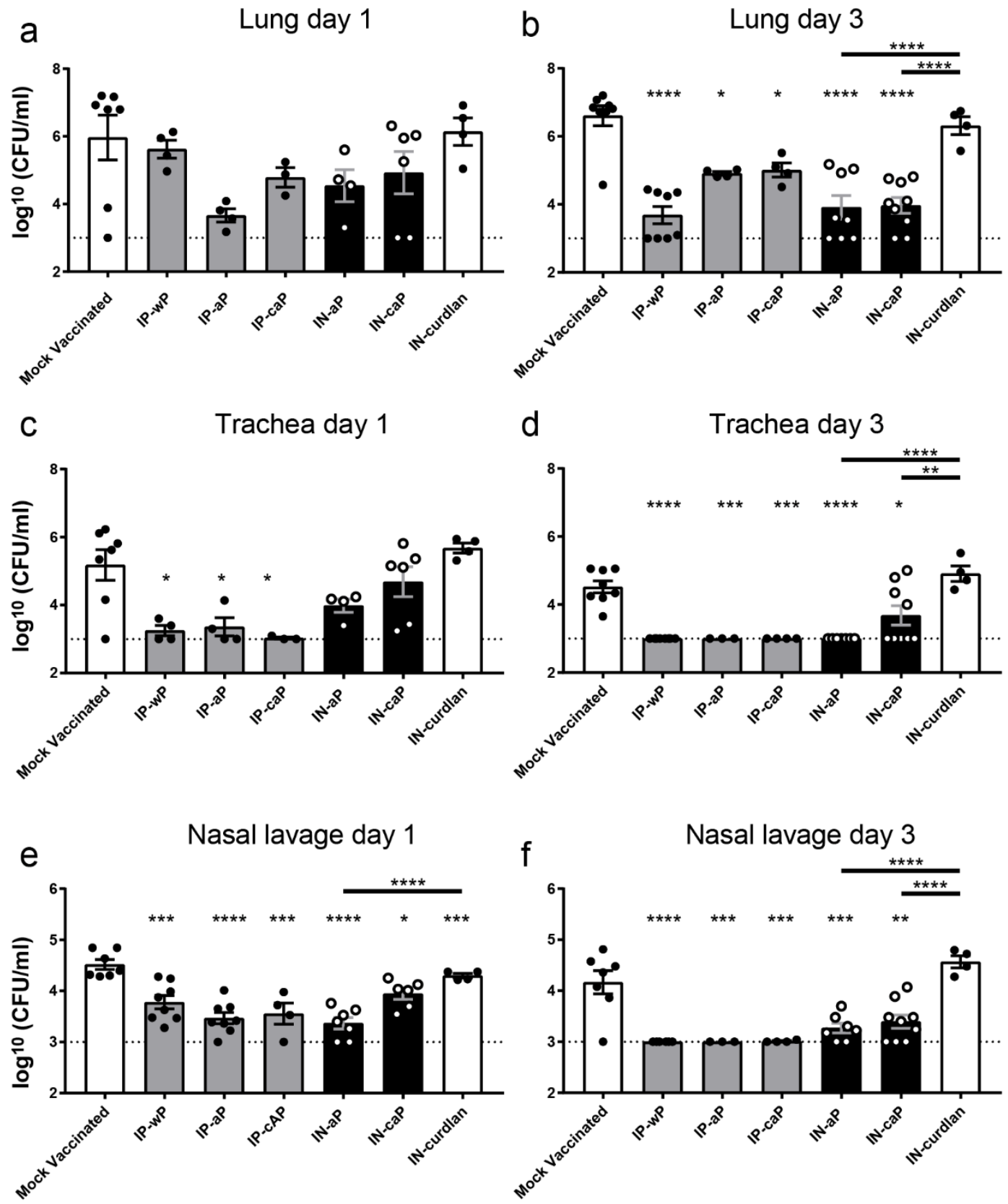

Figure 7: Intranasal immunization reduced respiratory $B$. pertussis bacterial burden. Analysis of bacterial burden was determined at days 1 and $3 \mathrm{pc}$. Bacteria were quantified by counting of serially diluted CFUs following immunization and challenge. 
CFU counts were determined from lung homogenate (A and B), trachea homogenate (C and D), and nasal lavage fluid (E and F). Results are mean \pm SEM $(n=4-8$, with 4 averaged technical replicates) from two independent experiments. ${ }^{\star} P<0.05$, ${ }^{\star \star} P<$ $0.01,{ }^{* \star \star} P<0.001$, ${ }^{* \star \star \star} P<0.0001$. $P$ values were determined by one-way ANOVA with Tukey's post hoc test compared to mock vaccinated mice, or between connected columns. The dashed line represents the lower limits of detection due to plating. 


\section{$\underline{\text { References }}$}

1. Mattoo, S. \& Cherry, J. D. Molecular pathogenesis, epidemiology, and clinical manifestations of respiratory infections due to Bordetella pertussis and other Bordetella subspecies. Clin Microbiol Rev 18, 326-382 (2005).

2. Melvin, J. A., Scheller, E. V., Miller, J. F. \& Cotter, P. A. Bordetella pertussis pathogenesis: Current and future challenges. Nat. Rev. Microbiol. 12, 274-288 (2014).

3. Kapil, P. \& Merkel, T. J. Pertussis vaccines and protective immunity. Curr. Opin. Immunol. 59, 72-78 (2019).

4. Rubin, K. \& Glazer, S. The pertussis hypothesis: Bordetella pertussis colonization in the pathogenesis of Alzheimer's disease. Immunobiology 222, 228-240 (2017).

5. CDC \& Baldy, S. W. R. L. M. Manual for the surveillance of vaccine-preventable diseases. (Centers for Disease Control and Prevention, 2015).

6. Pittman, M. History of the development of pertussis vaccine. Dev Biol Stand 73, 13-29 (1991).

7. Hill Elam-Evans LD, Yankey D, Singleton JA, Kang Y., H. A. Vaccination Coverage Among Children Aged 19-35 Months - United States, 2017. MMWR Morb Mortal Wkly Rep $2018 \quad 1123-1128 \quad$ (2017). doi:http://dx.doi.org/10.15585/mmwr.mm6740a4External

8. Organization, W. H. Pertussis Vaccines: WHO position paper-August 2015. 145168 (2018).

9. Klein, N. P., Bartlett, J., Fireman, B. \& Baxter, R. Waning Tdap Effectiveness in Adolescents. Pediatrics 137, e20153326-e20153326 (2016).

10. Klein, N. P., Bartlett, J., Rowhani-Rahbar, A., Fireman, B. \& Baxter, R. Waning Protection after Fifth Dose of Acellular Pertussis Vaccine in Children. N. Engl. J. Med. 367, 1012-1019 (2012).

11. Klein, N. P., Bartlett, J., Fireman, B., Rowhani-Rahbar, A. \& Baxter, R. Comparative Effectiveness of Acellular Versus Whole-Cell Pertussis Vaccines in Teenagers. Pediatrics 131, e1716-e1722 (2013).

12. Williams, M. M. et al. Bordetella pertussis strain lacking pertactin and pertussis toxin. Emerg. Infect. Dis. 22, 319-322 (2016).

13. Althouse, B. M. \& Scarpino, S. V. Asymptomatic transmission and the resurgence of Bordetella pertussis . BMC Med. 13, 146 (2015).

14. Ballister, E. R., Lai, A. H., Zuckermann, R. N., Cheng, Y. \& Mougous, J. D. In vitro self-assembly of tailorable nanotubes from a simple protein building block. Proc. Natl. Acad. Sci. 105, 3733-3738 (2008). 
15. Higgs, R., Higgins, S. C., Ross, P. J. \& Mills, K. H. G. Immunity to the respiratory pathogen Bordetella pertussis . Mucosal Immunol. 5, 485-500 (2012).

16. Mills, K. H. G. Immunity to Bordetella pertussis . Microbes Infect. 3, 655-677 (2001).

17. Edwards, K. M. et al. Comparison of 13 Acellular Pertussis Vaccines: Overview and Serologic Response. Pediatrics 96, 548-557 (1995).

18. Ausiello, C. M., Urbani, F., la Sala, A., Lande, R. \& Cassone, A. Vaccine- and antigen-dependent type 1 and type 2 cytokine induction after primary vaccination of infants with whole-cell or acellular pertussis vaccines. Infect. Immun. 65, 21682174 (1997).

19. Ross, P. J. et al. Relative contribution of Th1 and Th17 cells in adaptive immunity to Bordetella pertussis : towards the rational design of an improved acellular pertussis vaccine. PLoS Pathog 9, e1003264 (2013).

20. Misiak, A., Wilk, M. M., Raverdeau, M. \& Mills, K. H. G. IL-17-Producing Innate and Pathogen-Specific Tissue Resident Memory y $\delta \mathrm{T}$ Cells Expand in the Lungs of Bordetella pertussis -Infected Mice. J. Immunol. 198, 363-374 (2017).

21. Wilk, M. M. et al. Lung CD4 Tissue-Resident Memory T Cells Mediate Adaptive Immunity Induced by Previous Infection of Mice with Bordetella pertussis . J. Immunol. 199, 233-243 (2017).

22. Goodman, Y. E., Wort, A. J. \& Jackson, F. L. Enzyme-linked immunosorbent assay for detection of pertussis immunoglobulin $A$ in nasopharyngeal secretions as an indicator of recent infection. J. Clin. Microbiol. 13, 286-292 (1981).

23. Tuomanen, E. I., Zapiain, L. A., Galvan, P. \& Hewlett, E. L. Characterization of antibody inhibiting adherence of Bordetella pertussis to human respiratory epithelial cells. J. Clin. Microbiol. 20, 167 (1984).

24. Wolfe, D. N., Kirimanjeswara, G. S., Goebel, E. M. \& Harvill, E. T. Comparative Role of Immunoglobulin A in Protective Immunity against the Bordetellae. Infect. Immun. 75, 4416 (2007).

25. Solans, L. et al. IL-17-dependent SIgA-mediated protection against nasal Bordetella pertussis infection by live attenuated BPZE1 vaccine. Mucosal Immunol. 11, 1753-1762 (2018).

26. Gringhuis, S. I. et al. Dectin-1 directs $\mathrm{T}$ helper cell differentiation by controlling noncanonical NF-[kappa]B activation through Raf-1 and Syk. Nat Immunol 10, 203-213 (2009).

27. Agrawal, S., Gupta, S. \& Agrawal, A. Human dendritic cells activated via dectin-1 are efficient at priming th17, cytotoxic CD8 T and B cell responses. PLoS One 5, e13418 (2010).

28. Higashi, T. et al. Curdlan Induces DC-Mediated Th17 Polarization via Jagged1 Activation in Human Dendritic Cells. Allergol. Int. 59, 161-166 (2010). 
29. Goodridge, H. S., Wolf, A. J. \& Underhill, D. M. B-Glucan Recognition By the Innate Immune System. Immunol. Rev. 230, 38-50 (2009).

30. Carbonetti, N. H. Pertussis toxin and adenylate cyclase toxin: Key virulence factors of Bordetella pertussis and cell biology tools. Future Microbiol. 5, 455-469 (2010).

31. Boehm, D. T. et al. Evaluation of adenylate cyclase toxoid antigen in acellular pertussis vaccines using a Bordetella pertussis challenge model in mice. Infect. Immun. IAI.00857-17 (2018). doi:10.1128/IAI.00857-17

32. Wilk, M. M. et al. Immunization with whole cell but not acellular pertussis vaccines primes CD4 TRM cells that sustain protective immunity against nasal colonization with Bordetella pertussis . Emerg Microbes Infect 8, 169-185 (2019).

33. Wilk, M. M. et al. Lung CD4 Tissue-Resident Memory T Cells Mediate Adaptive Immunity Induced by Previous Infection of Mice with Bordetella pertussis . J. Immunol. 199, 233-243 (2017).

34. Allen, A. C. et al. Sustained protective immunity against Bordetella pertussis nasal colonization by intranasal immunization with a vaccine-adjuvant combination that induces IL-17-secreting TRM cells. Mucosal Immunol. (2018). doi:10.1038/s41385-018-0080-x

35. Solans, L. et al. IL-17-dependent SIgA-mediated protection against nasal Bordetella pertussis infection by live attenuated BPZE1 vaccine. Mucosal Immunol. 11, 1753-1762 (2018).

36. Zurita, M. E. et al. A Pertussis Outer Membrane Vesicle-Based Vaccine Induces Lung-Resident Memory CD4 T Cells and Protection Against Bordetella pertussis , Including Pertactin Deficient Strains. Front. Cell. Infect. Microbiol. 9, 125 (2019).

37. Ross, P. J. et al. Relative contribution of Th1 and Th17 cells in adaptive immunity to Bordetella pertussis : towards the rational design of an improved acellular pertussis vaccine. PLoS Pathog 9, e1003264 (2013).

38. Zhang, X., Goel, T., Goodfield, L. L., Muse, S. J. \& Harvill, E. T. Decreased Leukocyte Accumulation and Delayed Bordetella pertussis Clearance in IL-6-/Mice. J. Immunol. 186, 4895-4904 (2011).

39. Carbonetti, N. H. Contribution of pertussis toxin to the pathogenesis of pertussis disease. Pathog. Dis. 73, ftv073 (2015).

40. Mc Guirk, P., Mahon, B. P., Griffin, F. \& Mills, K. H. G. Compartmentalization of T cell responses following respiratory infection with Bordetella pertussis : hyporesponsiveness of lung $T$ cells is associated with modulated expression of the co-stimulatory molecule CD28. Eur. J. Immunol. 28, 153-163 (1998).

41. Barnard, A., Mahon, B. P., Watkins, J., Redhead, K. \& Mills, K. H. G. Th1/Th2 cell dichotomy in acquired immunity to Bordetella pertussis : Variables in the in vivo priming and in vitro cytokine detection techniques affect the classification of T-cell subsets as Th1, Th2 or Th0. Immunology 87, 372-380 (1996). 
42. Redhead, K., Watkins, J., Barnard, A. \& Mills, K. H. G. Effective immunization against Bordetella pertussis respiratory infection in mice is dependent on induction of cell-mediated immunity. Infect. Immun. 61, 3190-3198 (1993).

43. Baumann, E. et al. Development and clinical use of an oral heat-inactivated whole cell pertussis vaccine. Dev Biol Stand 61, 511-516 (1985).

44. Skerry, C. M. \& Mahon, B. P. A live, attenuated Bordetella pertussis vaccine provides long-term protection against virulent challenge in a murine model. Clin Vaccine Immunol 18, 187-193 (2011).

45. Thorstensson, R. et al. A phase I clinical study of a live attenuated Bordetella pertussis vaccine - BPZE1; a single centre, double-blind, placebo-controlled, dose-escalating study of BPZE1 given intranasally to healthy adult male volunteers. PLoS One 9, e83449 (2014).

46. Locht, C. et al. Live Attenuated Pertussis Vaccine BPZE1 Protects Baboons Against Bordetella pertussis Disease and Infection. J. Infect. Dis. 216, 117-124 (2017).

47. Allen, A. C. et al. Sustained protective immunity against Bordetella pertussis nasal colonization by intranasal immunization with a vaccine-adjuvant combination that induces IL-17-secreting TRM cells. Mucosal Immunol. (2018). doi:10.1038/s41385-018-0080-x

48. Brickman, T. J. \& Armstrong, S. K. The ornithine decarboxylase gene odc is required for alcaligin siderophore biosynthesis in Bordetella spp.: Putrescine is a precursor of alcaligin. J. Bacteriol. 178, 54-60 (1996).

49. Stainer, D. W. \& Scholte, M. J. A Simple Chemically Defined Medium for the Production of Phase I Bordetella pertussis . J. Gen. Microbiol. 63, 211-220 (1970). 


\section{Chapter 5 - Discussion}

Pertussis is a bacterial disease that only exists in the human population. Thousands of years of evolution have optimized $B$. pertussis to the microenvironment of the human respiratory system. This is understandably so, Homo sapiens are a species that fit the niche of $B$. pertussis. We are a social species that live in close quarters thereby permitting the dissemination of bacteria that spreads through respiratory droplets. Human offspring are born without fully developed immune symptoms, leaving them more susceptible compared to their adult counterparts. When one individual becomes sick others congregate to care for that person, more so in the case of an infant. Thus, giving rise for bacteria to spread to a new host and continue the infectious cycle.

However, the same forces of evolution that lead to the specialized niche of $B$. pertussis in the human respiratory system, confined the bacteria to life in only one host reservoir. As, scientists this is a flaw we can exploit, and have done so in the past. When Edward Jenner developed the smallpox vaccine, he initiated a mechanism of eradication of the disease by protecting the only species capable of spreading the smallpox virus. In 1980, natural occurring smallpox was declared eradicated through years of widespread wholecell vaccination ${ }^{1}$. It may now be surprising to some that pertussis, now classified as a re-emerging infectious disease by the CDC in the United States, was once a proposed target for eradication in the 1970 s $^{2,3}$. Like smallpox, pertussis incidence was wellcontrolled though widespread immunization with a wP vaccine ${ }^{4}$. The resurgence of pertussis began to occur in the US in the mid-1980s, and cases have remained well above that level since. This increase in cases coincides with the switch to aP in developed countries. One of the hypotheses on why this increase is occurring is that aP immunization inhibits the toxin-mediated symptoms the disease, but not colonization of the bacteria. This realization has renewed interest in the development of a vaccine that can inhibit colonization of $B$. pertussis, with the ultimate goal of reducing the number of 
cases to levels seen in the wP era, thereby re-opening the door the possible eradication of pertussis.

A major long-term goal of this work was to make progress on developing a vaccine that improved upon the shortcomings of current acellular pertussis vaccines covered in chapter 1. In this work we demonstrate the protective effects of neutralizing ACT, a key

virulence factor of $B$. pertussis, through an IP administered vaccine. We then explored another route of administration, finding that IN administration of DTaP induced a systemic protective response and a mucosal immune response in the respiratory tissue. This work demonstrates that the neutralization of toxins achieved through parenteral vaccination can be reciprocated by vaccinating the nasal cavity directly, thereby inhibiting PT before it disseminates beyond the respiratory system. This work suggests that the mechanisms of protection in either of the vaccine strategies should be further elucidated and incorporated into future pertussis vaccines.

\section{Lessons learned from Ne Cre luc mouse model}

The mouse model has been used for vaccine optimization since the development of wP vaccines ${ }^{5}$. Naturally, along the way, improvements to the mouse model have enabled a better understanding of vaccine-induced protection. In setting out to improve the current vaccines, we aimed to design experiments that were capable of showing this improvement. In a landmark study, Guiso et al. developed the intranasal pertussis challenge murine model ${ }^{6}$. In that study, the authors found similar protection from a dose of $1 / 4^{\text {th }}$ and $1 / 10^{\text {th }}$ of the human antigen concentration compared to a group receiving the full human dose ${ }^{6}$. We based our initial experiments on these vaccine concentrations.

One of the aims of chapter 2, was to use the NeCre luc, neutrophil reporter mouse model to trace the real-time kinetics of the neutrophil response between DTaP and WP immunized mice. In these experiments, we immunized the reporter mice with $1 / 5^{\text {th }}$ the human dose of DTaP and wP. At this high vaccine dose, we observed an aggressive pro-inflammatory response following $B$. pertussis challenge of WP immunized compared to DTaP immunized mice (Chap. 2, Figure 4,5). Transcriptional analysis of the lung during early and late phases of an acute infection revealed the highest pro-inflammatory 
associated gene changes in the wP-primed mice. Strikingly, we observed the highest levels of neutrophil recruitment occurred after the bacterial burden was cleared to limits of detection, while in comparison to the DTaP-primed group the percentage of neutrophils in the lung had returned to baseline levels following clearance of the infection (Chap. 2, Figure 3,7). The intensity of the neutrophil response may have overwhelmed this group, as we observed severe morbidity in wP-primed mice at day 6 , which required euthanasia. The clearance of the pathogen prior to the recruitment of neutrophils suggests that morbidity was due to the immune response elicited. The NeCre luc model provided a novel method of visualizing the spatiotemporal localization of neutrophils in immunized mice. In these studies, we compared the quantification of neutrophils using the luminescence of the luciferase-induced reporter to the quantification of neutrophils through flow cytometry. We determined that using flow cytometry we could accurately depict the neutrophil response over time, with the benefit of using a more standard mouse strain. The NeCre luc reporter mouse may be the most beneficial to those study the neutrophils recruited to a concentrated site, such as those studying the role of innate immune cells in the tumor microenvironment, or acute topical infections. In completing this aim of the study, we concluded that a WP vaccine concentration of $1 / 5^{\text {th }}$ the human dose may be too high to properly characterize the response of neutrophils overtime.

A second aim of the NeCre luc study was to determine the protective role of a purified toxoid of ACT, RTX, as a single antigen vaccine administered with aluminum hydroxide. We hypothesized that antibodies generated against the antigen would neutralize the toxin activity of ACT, thereby inhibiting the ACT mediated suppression of neutrophils discussed in chapter 1. RTX immunization generated a strong IgG antibody response specific to the RTX antigen; however, RTX as a single antigen vaccine alone was not protective because we did not observe a reduction of bacterial burden in these experimental conditions. Immunization with only RTX would not address PT, thus in these experiments, PT would still be active. Thereby, inhibiting neutrophil and macrophage chemotaxis to the site of infection. We reasoned that while RTX as a single antigen did not induce protection the benefits of neutralizing ACT may be more evident when PT is neutralized as well. In this scenario when macrophages and neutrophils 
were recruited to the lung, their bactericidal activity would not be inhibited. This realization caused us to re-craft our approach to a vaccine where we could study the synergistic effects of neutralizing ACT, along with the protective activity of DTaP vaccines.

\section{Optimization of the mouse model}

In chapter 3, we built upon our observations learned in the NeCre luc immunization and challenge model. In these experiments we aimed to determine the protective effects of incorporated RTX in a DTaP vaccine. In initial experiments, we included the RTX toxoid discussed in chapter 2 , into a $1 / 5^{\text {th }}$ human dose of DTaP. In these results, we measured an antigen-specific immune response but did not observe an increase in protection compared to DTaP immunized mice. It occurred to us, that the use of such a high dose of vaccine in the mouse model is not relevant to the dose that is administered to humans. This reflection led us to the idea that to establish a relevant model, we needed to determine the lowest vaccine dose that induced protection. We proposed performing a vaccine dose titration to find this dose in mice. This would lead to a vaccine dose that would: 1) more accurately depict the vaccine concentration administered to humans, and 2) establish a sub-protective dose to incorporate future vaccine antigens, such as RTX to determine protection. In chapter 3 , we determined that $1 / 40^{\text {th }}$ of the human dose of DTaP vaccine protected mice while, the $1 / 80^{\text {th }}$ dose did not. We were then able to add RTX to $1 / 80^{\text {th }}$ human dose DTaP vaccine. This finding does correlate with others suggesting that $1 / 50^{\text {th }}$ the human dose of DTaP was protective in mice, as we identified $1 / 40^{\text {th }}$ the human dose to be the lowest-protective dose by our 2-fold titration 7,8 . Defining this sub-optimal dose is crucial in re-thinking the design of DTaP/TdaP immunizations. DTaP/TdaP are safe vaccines which protect individuals from classical pertussis. Therefore, if a potential vaccine is to replace either of these standards, the candidate vaccine would need to perform better, in addition to protecting an individual through a different mechanism. Defining a sub-optimal vaccine dose from which, synergistic effects of potential vaccine antigens can be observed, gives researchers a mechanism to demonstrate increased vaccine potency. 


\section{Incorporation of RTX with DTaP}

In chapter 1, we discussed the immunomodulatory activity of ACT. ACT increases intracellular CAMP of macrophages and neutrophils recruited to the site of infection. The levels of CAMP suppress the bactericidal activity of the macrophages and neutrophils including phagocytosis, oxidative burst and NET formation in neutrophils ${ }^{9-11}$. We, along with others hypothesized that neutralizing ACT through vaccination would increase vaccine efficacy of current acellular vaccines ${ }^{12,13}$. In chapter 3 , we discussed the protective effects of including RTX into a DTaP vaccine. In these studies, we immunized mice using the outbred CD-1 mouse line. Incorporation of RTX into the DTaP vaccine resulted in the production of anti-ACT binding antibodies, that were capable of neutralizing ACT activity against murine macrophages (Chap. 3, Fig. 1,3). These in vitro observations translated to in vivo vaccine-mediated protection, as we observed decreased bacterial burden and pro-inflammatory responses with the inclusion of RTX compared to DTaP alone (Chap. 3, Fig. 2). Defining the sub-optimal vaccine dose was critical to this experiment. We observed synergic effects of RTX at $1 / 80^{\text {th }}$ human vaccine dose; while those effects were masked at the higher doses of DTaP (Chap. 3, Fig. 1).

We observed that the inclusion of RTX into the DTaP vaccine increased vaccineinduced protection when compared to DTaP alone. We did not observe an increase in Th1 associated cytokines, suggesting that DTaP with RTX did not alter the vaccineinduced immune response from the Th2 promoting DTaP. Overall, the immune response to $B$. pertussis was similar to DTaP induced protection. We observed strong IgG responses to the $B$. pertussis toxins, and a reduction in the pro-inflammatory response evident by a reduction in pro-inflammatory cytokines, macrophages, and neutrophils in the lung when RTX was included in DTaP compared to DTaP alone. The data suggests that the enhanced protection was due to the production of anti-ACT neutralizing antibodies. However, due to the nature of the Th2 response observed from DTaP with RTX, it seems unlikely that the inclusion of an additional antigen alone would increase the duration of protection compared to DTaP. We hypothesized that to alter the T-helper cell response and increase the duration of protection, we needed to continue investigating ways to promote a cell-mediated immune response. 
The work performed in this dissertation was completed in the mouse model of pertussis. The use of animal models has vastly contributed to the understanding of the pathogenesis and the immune response elicited against $B$. pertussis and other Bordetella species. Mice and humans generate similar pro-inflammatory T-cell responses and antibody profiles upon infection with $B$. pertussis ${ }^{14}$. In chapters 3 and 4 , we utilized only female CD-1 outbred mice, compared to inbred mouse strains. The use of inbred mouse strains increases the reproducibility of experiments compared to experiments using outbred mice strains. Outbred strains were used in our experiments specifically to increase the genetic variability, to model the genetic variability seen in the human population. As a result, we observed increased variation in experiments performed using CD-1 mouse lines compared to inbred strains such as BALB/c (data not shown). In addition to genetic variability between mouse strains, future experiments should utilize both male and female sexes. Epidemiology pertussis studies have found a higher proportion of pertussis in female patients compared to male counterparts ${ }^{15}$. Classically, females are more susceptible to upper respiratory infection compared to males $^{16}$. However, females are known to induce greater innate immune cell activation and stronger humoral responses than males in both human and mice ${ }^{17}$. It is possible that male mice may not reciprocate the strength of the immune response observed following immunization and challenge of female mice. In future experiments the incorporation of both male and female mice can address this concern.

\section{Importance of a Mucosal Response against B. pertussis}

In our studies, we found it interesting that bacteria were cleared earliest in the lungs compared to nares and trachea (Chap 3., Fig. 2). We began to investigate the possibility of a mucosal pertussis vaccine that could prime the respiratory tract directly. We hypothesized that by administering the vaccine intranasally, the primed local immune response could neutralize the pathogen before the impairment of the innate immune response by toxins of $B$. pertussis.

Mucosal-vaccine induced immunity poses the benefit of preventing the establishment of an infection prior to PT entering the circulatory system. Conversely, parenteral pertussis vaccine-induced immunity requires circulating $\mathrm{PT}$ to encounter memory $\mathrm{T}$ and $\mathrm{B}$ cells 
present in the draining lymph nodes, then mount a secondary immune response to the infection. It is well documented that natural infection of a human patient confers longterm protection. While the human mucosal immune response is yet to be characterized, B. pertussis-specific IgA antibodies suggest a role for mucosal protection ${ }^{18}$. Evidence in support of a role of a mucosal immune response against $B$. pertussis from the baboon model find that convalescent baboons and mice clear the infection significantly earlier than any parental vaccine, WP or aP ${ }^{19,20}$. The natural response to pertussis was discussed in chapter 1 , but is known that natural infection with $B$. pertussis induces a strong mucosal response, which correlates to sterilizing immunity ${ }^{21,22}$. Wendelboe et al., reviewed published data comparing the duration of protection from acellular and WP pertussis vaccines to convalescent protected individuals ${ }^{23}$. The authors determined that humans who were previously infected with $B$. pertussis were protected up to 20 years, compared to up to 12 years following WP immunization, and 4 years from those immunized with various DTaP vaccines ${ }^{23}$. We posed the question, "If we can mimic an infection with an acellular vaccine, could we induce a protective response?" Now, there are clear differences between an infection with pathogenic bacteria and a vaccine composed of purified protein. For one, not only does the pathogen express thousands of potential PAMPS, but the colonization of the respiratory tract produces additional damage-associated molecular patterns (DAMPS) that would be not produced following intramuscular acellular immunization. PAMPS/DAMPS associated with the natural infection recruit cells of the innate immune system to the infection, leading to phagocytosis of the bacteria, and ultimately antigen presentation of the many potential antigens to naïve $T$ cells ${ }^{24,25}$. This is unlikely to occur with a parenterally administered subunit vaccine, where a humoral response is generated against the specific antigens contained in the vaccine.

As discussed previously, there has been promising pre-clinical success with a liveattenuated $B$. pertussis intranasal vaccine, BPZE1 ${ }^{26-29}$. BPZE1 is a highly attenuated strain, lacking production of three functional virulence factors of $B$. pertussis: PT, dermonecrotic toxin, and tracheal cytotoxin ${ }^{29}$. The strain is not pathogenic but is able to colonize the respiratory tract, before eventually cleared. The vaccine strain is protective in mice and baboons, and in mice has been shown to induce a Th1 mediated response 
$26,29,30$. Recently, it has shown that immunization with this strain resulted in production of IL-17, IgA antibodies, and the TRM population in the nares of mice, each of these factors was shown to play a role in protection ${ }^{28}$. The work performed by Solans et al. further demonstrates the importance of a local mucosal immune response in reducing colonization of the respiratory tract, and of generating a pro-inflammatory cell-mediated response in long-term protection against $B$. pertussis.

\section{Intranasal DTaP induced a protective immune response}

In chapter 4 , we changed the route of vaccine administration from an intraperitoneally administered vaccine to an intranasally administered vaccine. In doing so, going from a vaccine response that was well characterized to the relativity unexplored vaccine route: mucosal vaccination ${ }^{8,22,31-33}$. One of the challenges we were faced with was the number of potential antigens that encounter the nasal-associated lymphoid tissue (NALT) on a daily basis ${ }^{34}$. Data from orally administered vaccines demonstrates that gut-associated lymphoid tissue promotes tolerance over immunogenicity ${ }^{35}$. We reasoned that because the alum adjuvant is not optimal for mucosal vaccination, that IN administered DTaP may not induce enough inflammation to induce a protective immune response ${ }^{36}$. It may be that in order to induce a systemic response, the vaccine would have to stimulate a pro-inflammatory response upon vacation, as was the case in the BPZE1 vaccine discussed above. To increase the pro-inflammatory properties of DTaP, we supplemented DTaP with the $\beta$-glucan curdlan. Curdlan was an attractive candidate as it had been previously tested as an intranasal adjuvant, and has been shown to promote a Th1/Th17 immune response in vitro and in vivo ${ }^{37-40}$. Specifically, curdlan binds to the fungal receptor dectin-1 expressed on the surface of DCs, found at high concentrations in the NALT, along with macrophages ${ }^{41,42}$. Also, lining the mucosal surface are microfold (M) cells, which specialize in phagocytosis of macromolecules, viruses, and bacteria. On the basal surface of the $M$ cells are intraepithelial pockets, which can contain DCs, $T$ and B cells ${ }^{34,41}$. APCs from the mucosal tissue can then track to the draining lymph nodes, where they activate B cells, ideally inducing a systemic and mucosal immune response ${ }^{34}$. 
Mucosal immunizations provide a unique vaccination strategy because antigen priming can occur in multiple lymphoid organs and tissues. Furthermore, the appropriate tissue may change based on the type of vaccine and/or depending on the pathogen. In the case of pertussis, the appropriate tissue for vaccination is yet to be determined and all human vaccines are provided by IM injection. Since we aimed to vaccinate the airway, we thought it was necessary to track where the vaccine is going during IN immunization. In chapter 4, we accomplished this by fluorescently labeling the DTaP vaccine particles. In Chap. 4, Fig 1,2, we observed that the majority of DTaP alone (IN-aP) was deposited in the lung while DTaP with curdlan (IN-caP) was found mostly in the nares. Ultimately, we did not determine a difference in B. pertussis-specific IgG antibody titers present in the serum or IgA antibodies in the lung supernatant. Suggesting that whether antigen uptake occurred in the lungs or the nasal cavity, IN immunization elicited a systemic response not 


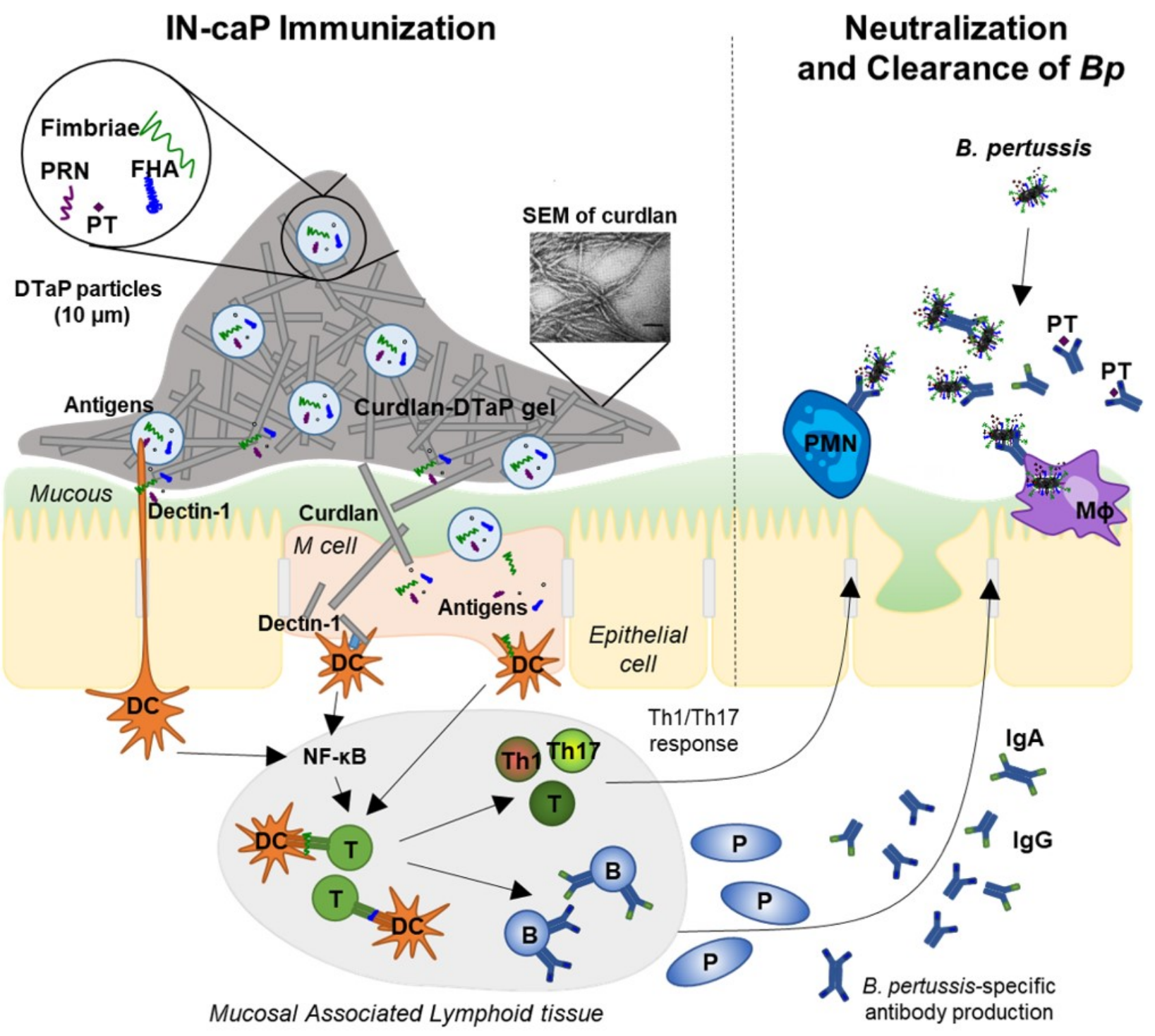

Figure 1: Proposed model of IN-caP induced protection.

The fungal polysaccharide curdlan is administered as a semi-gel state at a neutral $\mathrm{pH}$. The immunostimulant properties of the $\beta$-glucan elicit a Th1/Th17 response. Curdlan binds to DCs through the dectin-1 receptor. The dectin-1 signaling pathway induces the non-canonical expression of NF-kB, promoting a Th1/Th17 immune response. Curdlan as an adjuvant induced antigen-specific serum IgG antibodies, and IgA antibodies in the lungs of vaccinated mice. 
significantly different than IP. Furthermore, the inclusion did not further reduce the bacterial burden of the respiratory system (Chap. 4, Fig. 7). In these experiments we demonstrated that the IN administration of DTaP could induce the production of IgA antibodies. It is then possible that antibodies found in the lung homogenate and nasal lavage fluid could be neutralizing PT at the site of infection. Thus, leading the lower levels of pro-inflammatory cytokines found in the lung homogenate. These data suggest that IN-aP immunization alone is capable of generating a systemic and mucosal immune response.

\section{Role of curdlan in a mucosal immune response}

Curdlan has been shown to induce Th17 responses when administered through the intranasal route ${ }^{37}$. In this study, we determined the highest amount of IL-17A in the lungs of groups that were IN immunized, with the DTaP with curdlan inducing the highest levels (Chap. 4. Fig. 5d). However, while significant, this response is not as strong as was observed following WP-induced IL-17A response as we demonstrated in this and other studies. What is interesting is that when we measured DTaP vaccine particles in the nares of the mice, more appeared to be embedded in the nasal cavity epithelium with IN-caP than compared to more particles in the lumen with IN-aP (Chap. 4, Fig. 2c). Furthermore, the particles from IN-caP had a smaller, more, particulate appearance. It may be that curdlan played a role in antigen uptake in the nasal cavity. In this study, we only observe a correlation between the smaller particles in the mucosal tissue and the increased IL-17A production found in DTaP with curdlan. Future experiments will be needed to further elucidate this mechanism. One of the caveats of this experiment is that we did not identify the cell types that contained or bound vaccine particles in microscopy imaging. It is possible that when DTaP was administered with curdlan, the vaccine particles were more likely to be phagocytosed by $M$ cells of the mucosa, which are known to endocytose particulate antigens ${ }^{43}$. Thereby, it may be possible that antigen uptake is more efficient through this mechanism. 


\section{Future directions in pertussis vaccine development}

In this work, we demonstrated the induction of a protective response from an intranasally administered vaccine. This work suggests that mucosal immunization with the current DTaP vaccine alone produces a systemic immune response and the production of IgA antibodies and IL-17A in the respiratory tract. The next question to answer is, what is the mechanism of protection elicited from DTaP and DTaP with curdlan? In order to develop effective mucosal pertussis vaccines, a better understanding of the mucosal immune response to $B$. pertussis is needed.

\section{Identification of target vaccine tissue}

The use of fluorescent vaccine particles gave us the ability to track the vaccine in the nasal cavity of mice. Presumably, antigen uptake and presentation could be explored using a similar experimental design that could identify $M$ cells and continued tracking of the vaccine particles. An experiment of this nature would also help to answer the question of appropriate tissue targeting. In our experiments, a volume of $50 \mu \mathrm{l}$ of the vaccine was administered intranasally. This volume is known to reach the lungs in the mice, while the use of lower volumes results in the majority of detection of bacteria or labeled protein accumulating in the upper respiratory system ${ }^{44,45}$. Thus, in the mouse model, this administration route has the potential of antigen priming occurring in the upper and lower respiratory system, as the dose is exposed to the two tissues. In performing the experiment mentioned above, one could elucidate whether either of the tissues plays a role in establishing the mucosal immune response. Additionally, upon antigen presentation either to $\mathrm{T}_{\mathrm{RM}}$ in the respiratory tissue or draining lymph node one would expect to measure a spike in pro-inflammatory cytokines following the immunization. Using a $B$. pertussis outer membrane vesicle vaccine (OMV) Raeven et al., measured the induction of a moderate pro-inflammatory response compared to WP immunization ${ }^{46}$. This response was measured in serum 4 hours post-boost immunization, resulting in significant increases of G-CSF, KC, CXCL10, IL-6, IL-1 $\beta$, IL5, MCP-1. Similarly, Warfel et al., measured increases of IL-6, IL-1 $\beta$, IL-23, G-CSF, MCP-1, and MIP-1x from nasopharyngeal washes from baboons following challenge with $B$. pertussis ${ }^{47}$. As early as 48 hours after challenge levels of IL-6 and G-CSF were 
significantly increased compared to negative control baboons. It may be beneficial to study the production of cytokines and chemokines produced early in the immune response to $B$. pertussis infection in the context of immunizations. The rationale being, that the production of cytokines associated with antigen presentation measured in NALT tissue or the draining lymph nodes could further elucidate the generation of the $B$. pertussis-specific immune response. In the mouse model it may not seem to be of consequence, but in human applications targeting of the appropriate tissue depends on the delivery method used in vaccination. In humans, nasal droppers, nasal sprays, nebulizers, and dry powders have been used for delivery of vaccines to the upper respiratory tract 34,48 . Vaccination of the lower respiratory system requires the use of a nebulizer-generated small-particle liquid or powder vaccines that are capable of reaching the lungs ${ }^{34,49}$. Understanding the differences in the immune response elicited between antigen priming occurring in either tissue will be crucial for the translation to non-human primate and ultimately clinical trials. 


\section{Mucosal Response to $B$. pertussis}

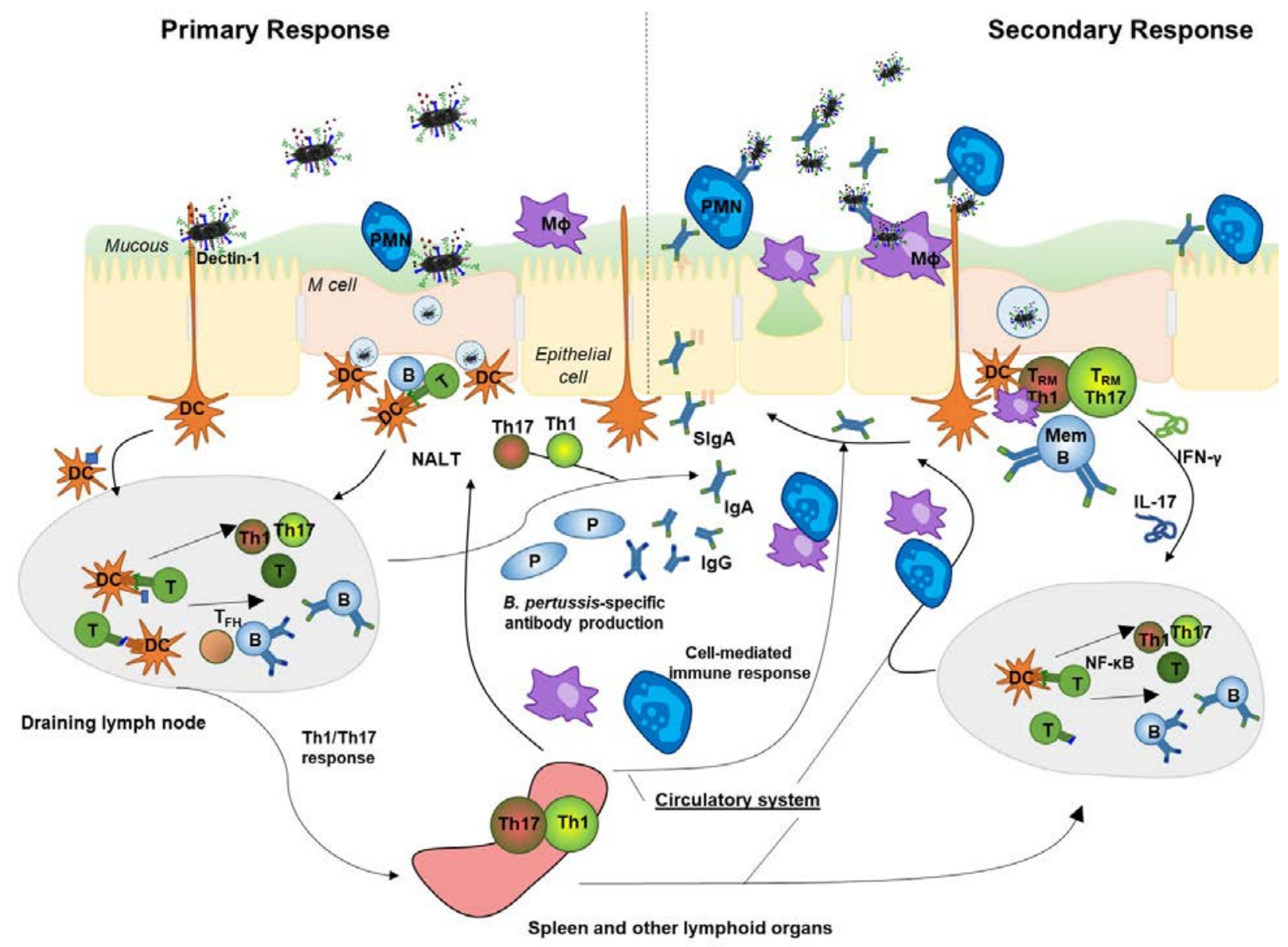

Figure 3: Summary of proposed mucosal immune response to B. pertussis.

During the primary infection with $B$. pertussis innate immune cells are recruited nasal cavity. Bacteria and potential antigens can be phagocytosed by DC cells embedded in the epithelium layers, once activated DCs migrate to the draining lymph nodes where they present antigen to the naive $T$ cells. Specialized lymphoid tissue in the nasal cavity, NALT, line the nasal cavity. These NALT tissues contain M cells that endocytosis bacteria or particulate antigen and translocate the organism or macromolecule to DCs, B and T cells present on the basal surface of the cells. Antigen priming of naïve T cells differentiate to Th1, and Th17 effector cells in the draining lymph nodes or NALT tissue. $T_{F H}$ cells activate $B$ cells which promote B. pertussis antigen specific IgG and IgA antibodies. Effector Th1 and Th17 migrate to respiratory tract, a subset of CD4 memory effector cells are retained in the tissue as $T_{R M}$. Effector Th1/Th17 cells recruit macrophages and neutrophils phagocytose the now opsonized bacteria. Upon secondary infection by B. pertussis $\mathrm{T}_{\mathrm{RM}}$ recognized the pathogen proliferating to effector Th1 and Th17 T cells, secreting IL-17 and IFN- $\gamma$. IL-17-secreting T cells have also been shown to play a critical role in the secretion of SIgA antibodies. The activation of the mucosal memory response immediately recruits macrophages and neutrophils to the infection, which with help from SlgA antibodies can efficiently phagocytosis B. pertussis. Thus, limiting the extent of the secondary infection. Figure adapted from Wilk 
et al. $^{50}$ Solans and Locht $^{51}$ and Neutra and Kozlowski ${ }^{41}$. PMN-polymorphonuclear neutrophils, M $\phi$ macrophage, B- Naïve B cells, T-Naïve T cell, NALT-Nasal associated lymphoid tissue, Th17-Th17 T helper cell, Th1-Th1 $T$ helper cell, DC-Dendritic cell, $T_{F H}-T$ follicular helper cell, $T^{R M}$ TH17- Tissue resident memory Th17 cell, $\mathrm{T}^{\mathrm{RM}} \mathrm{TH} 1-$ Tissue resident memory Th1 cell, Mem B- Antigen-specific Memory B cell

\section{Importance of nasal cavity specific analysis}

The findings presented in chapter 4 suggest that intranasal immunization is a viable strategy that needs to be further investigated. We and other groups are studying intranasal immunization against $B$. pertussis ${ }^{28,52}$. It is clear that to continue down this avenue of research, the correlates of protection of the local mucosal immune response need to be fully characterized. Until recently, the majority of pertussis research has focused on characterizing the immune response in the lower respiratory tract in the mouse model; however, except in rare cases, B. pertussis infects the upper respiratory system ${ }^{53}$. Wilk et al., have begun to focus on the immune response and bacterial clearance of the nasal cavity, specifically ${ }^{8}$. This approach involves the intricate process of removal of the epithelial lining for analysis by flow cytometry and bacterial burden. Through this method, they have identified the role of IL-17 in promoting nasal cavity SlgA and IL-17-secreting TRM cells in vaccine-induced protection ${ }^{8,28}$. The role of $T_{R M}$ in protection against $B$. pertussis is yet to be fully understood. Data exists that correlates the presence of the $\mathrm{T}_{\mathrm{RM}}$ cell population in the nasal cavity to long term protection, specifically in the nasal cavity of mice ${ }^{52}$. Currently, it is suggested that the production of IFN-y and IL-17 are essential to clearance in the nasal cavity, and data have shown the production of IFN- $\gamma$ and IL-17 from $T_{R M}$ and $\gamma \delta$ T cells in the nares 8,28,54. However, the mechanism of clearance remains to be discovered.

A closer examination of the nasal cavity, the bacterial burden in the nares can be more accurately depicted. The nasal cavity clearance data included in this dissertation, along with the work of others, quantify bacteria burden present in nasal lavage fluid ${ }^{14}$. This method quantifies bacteria that were removed from the upper respiratory system by the lavage; however, it is apparent that a significant portion of the bacteria remain ${ }^{52}$. In moving forward, techniques to more accurately quantify the bacterial burden in the nares will be necessary to establish true sterilizing immunity. The importance of clearance in the nares is emphasized by our findings that the infection in the lung was cleared, but not nasal cavity. The different anatomy and microenvironments of the lung 
and of the upper respiratory tract may play a role in bacterial adherence and colonization ${ }^{55}$. Findings by Conover et al. suggest that $B$. pertussis can potentially form biofilms in the mouse nose, while they did not in the mouse lungs; however, this is yet to be shown by other groups ${ }^{56}$. The authors found that the BPS polysaccharide of $B$. pertussis was responsible for colonization and promoted biofilm formation in the nasal cavity; but not in the lungs. Additionally, FHA has also been shown to promote biofilm formation and is critical for biofilm formation in the mouse nose and trachea ${ }^{57}$. Understanding of differences between the lower and upper respiratory system will be essential in determining why $B$. pertussis infection persists in the nasal cavity compared to the lower respiratory system. To observe these differences, experiments capable of addressing the mechanisms behind the immune responses between the tissues are needed.

In WP immunized and naïve mice we have measured robust recruitment of macrophages and neutrophils to the lungs (Chap. 2, Fig. 4, Chap. 3, Fig. 5,6, Chap. 4, Fig. 6). In these experiments, we determined the numbers of cells recruited to the infection; we did not address their functionality. It is possible that due to the Th1 response induced in convalescent or WP vaccine-induced protection APCs may more efficiently phagocytose bacteria. The experiments performed by Wilk et al. and Solans et al. have shown that IL-17 is critical for $B$. pertussis clearance from the nasal cavity. The mechanism; however, is yet to be determined. It would make the most sense to start with the roles of neutrophils in the nasal cavity, as IL-17 is known to induce recruitment of neutrophils.

\section{Neutrophils in the nasal cavity, the unsung hero?}

The question we are faced with is what changes between neutrophils in the mucosal response of an immune individual and that of a naïve individual? We have already discussed the first answer, that neutrophils have only been found to play a role in the clearance when bacteria are opsonized. We now know that IgA secreted through the mucosa can opsonize $B$. pertussis, presumingly leading to increased phagocytosis 28 . Another reason may be the timing of the neutrophil response. In a primary infection, there is a slight increase in neutrophils at day 1 ; however, the neutrophil response does 
not peak until days 5 and 7 post-infection ${ }^{58-61}$. It could be hypothesized that the IL-17 produced by $T_{R M}$ and $\gamma \delta T$ cells present in the nasal cavity, would recruit neutrophils to the nasal cavity earlier. Thus, decreasing the ability of $B$. pertussis toxins to inhibit phagocytosis of the bacteria. A third reason could be the neutralization of the toxins themselves. Secreted IgA and IgG present in the mucosa of a protected host could be inhibiting dysregulation of the innate immune response. The neutralization of PT alone has been shown in numerous studies to provide protection ${ }^{62,63}$. Neutralizing PT in the mucosa would increase neutrophil and macrophage recruitment to the site of infection, PT inhibits both macrophage and neutrophil recruitment to the infection ${ }^{64,65}$. Lastly, it was previously mentioned that $B$. pertussis biofilm formation could occur in the nasopharynx and trachea of a mouse ${ }^{66}$. Neutrophils produce NETS that trap bacteria in a "web" of DNA and proteins, destroying them through released enzymatic granules and proteases ${ }^{11}$. Recently Thanabalasuriar et al., demonstrating that NETS were able to confine $P$. aeruginosa biofilms in the ocular biofilms and prevent dissemination ${ }^{67}$. These mechanisms provide potential roles of neutrophil-mediated clearance in the mucosal response to $B$. pertussis; however, as mentioned above, experiments need to be performed on neutrophils of the nasal cavity.

\section{Neutralizing ACT in the nasal cavity}

In chapter 3 , we demonstrated the neutralization of ACT from serum of $a P+R T X$ immunized mice (Chap. 3, Fig. 1). ACT can inhibit many of the bactericidal actions of macrophages and neutrophils, as discussed in Chapter 1. Determining the effects of neutralizing ACT directly in the respiratory tract, could lead to a more complete inhibition of the "one-two punch" toxin activity of PT and ACT, thus increasing clearance of $B$. pertussis in the nasal cavity ${ }^{64}$. In chapter 3 , found that the inclusion of RTX into DTaP administered through IP injection reduced IL-6 production in the lung. (Chap 3, Fig. 4). IL-6 is a pleiotropic pro-inflammatory cytokine known to be increased upon $B$. pertussis infection, as we and others have observed a large increase in IL-6 following challenge of a naïve or wP protected mouse ${ }^{31,68}$. In our interpretation, a reduction of IL-6 correlates with DTaP-mediated protection. Interestingly, in chapter 4 , we observed the lowest levels of IL-6 in the lung when mice were immunized intranasally compared to intraperitoneally with IN-aP or IN-caP (Chap. 4, Fig 5.). Suggesting that priming the site 
of infection, rather than inducing priming in a distal lymph node and generating a systemic response induces a stronger response in the respiratory tract.

It would then appear that by administering RTX in an intranasal DTaP vaccine, one could combine the additive effects of neutralizing RTX, with the enhanced local response generated from an intranasal vaccine. One would suspect that these protective effects would be the most evident in the bactericidal activity of macrophages and neutrophils in the respiratory tract where the cells would encounter active ACT. In particular, we would hypothesize enhanced phagocytosis and bacterial killing in neutrophils and macrophages due to neutralizing ACT mediated inhibition of oxidative burst. Additionally, it is known that ACT can inhibit NET formation due to cAMP levels ${ }^{11}$. It would be interesting to measure NET production and bacteria mediated killing in the lungs and nasal cavity, although these fragile structures will require fine-tuning of protocols. Furthermore, there is potential for NET formation to reduce biofilm formation in the upper respiratory system. Again, these experiments will need to focus on the upper respiratory system, but the results could be pivotal in the generation of future pertussis vaccines.

\section{Potential future adjuvants}

Our work with curdlan adjuvant demonstrated a modest increase in IL-17A in the lungs of mice immunized with DTaP and curdlan compared to mice immunized with DTaP alone (Chap. 3, Fig. 5). However, we did not observe an increase in macrophages or neutrophils recruited to the lungs. This may be due to the fact that all DTaP groups still contain aluminum hydroxide, promoting a Th2 response that is masking the effects of curdlan. Experiments to further elucidate the effects of $\beta$-glucans without aluminum hydroxide are ongoing and have yielded positive initial results. Still, it may be that optimal adjuvant is still yet to be found. Studies examining TLR 2 and 9 agonists from the Kingston Mills group have had success in the mouse model inducing Th1/Th17 responses and increasing IgG2a antibodies ${ }^{14,69}$. The same group has combined a TLR2 agonist with c-di-GMP, an agonist for the intracellular receptor stimulator of interferon genes (STING). This parenteral vaccine has been shown to promote Th1/Th17 responses, and particularly a $B$. pertussis-specific $T^{\mathrm{RM}}$ response in the lungs. 
Importantly, this protective response was measured up to 10 months after immunization. Work isolating outer membrane vesicles (OMV) from $B$. pertussis has proven to be successful. $B$. pertussis OMVs and DT toxoids were shown to induce Th1/Th17 immune responses and increase the IgG2a/IgA ratio compared to a DTaP vaccine ${ }^{70}$. OMV vaccines are promising because they provide an increased proinflammatory response compared to DTaP; but, less reactogenicity than wP immunizations ${ }^{46,71}$. Particulate adjuvants are an interesting area of research as the particles may increase phagocytosis in the upper respiratory system, as discussed above. The combination of DTaP, CpG, and microparticles intranasally administered was shown to protect mice similar to levels observed in mice immunized with parenteral administered DTaP ${ }^{72}$. It is possible that the conjugation of toxoids to the particles could increase vaccine effectiveness in future vaccines. Defining an optimal adjuvant is crucial to the success of a mucosal pertussis vaccine. The problem arises that boosting the immune response with a powerful adjuvant could have adverse effects and ultimately might also prevent licensure due to safety concerns.

\section{Mucosal pertussis booster}

It is because of this balancing act that the commercial producers of pertussis vaccines are at an impasse. DTaP/Tdap do prevent a large number of potential cases of whooping cough but these vaccines are not as effective as the wP vaccines in regard to the durability of protection. The use of the DTaP/Tdap vaccine strategy does protect the majority of those who receive the vaccine from clinical pertussis. It is clear that shortcomings exist with DTaP/Tdap vaccines, but the question remains, what is the alternative? DTaP/Tdap vaccines were originally designed to be safer vaccines capable of inducing similar protection to that of their WP predecessors, and they have. After completing the $5^{\text {th }}$ dose DTaP protection ranges from $80-90 \%$ effectiveness ${ }^{2}$. Data from adolescents suggest that the problem exists in years following the completion of DTaP 73. According to ClinicalTrials.gov, there are currently 318 clinical trials either ongoing, recently completed or upcoming studying pertussis, of those 62 are focused on booster immunizations. However, most of these focus on safety and formulation of currently available immunizations. It seems that the vaccine industry is reluctant to replace current vaccines due to effectiveness in preventing disease and their safety. However, 
there is potential for replacement of Tdap with a booster vaccine capable of inducing a Th1/Th17 response. The problem, as eluded to in chapter 1 , is that vaccine imprinting of the Th1 primary response induced from wP in humans promotes the same response even after DTaP booster immunizations ${ }^{74}$. This dilemma provides the strongest support for the development of an intranasal vaccine. The hypothesis being that parenteral booster immunizations are recognized by the memory $T$ and $B$ cells generated from the parenteral priming immunization. In altering the route of administration to the intranasal route, and with a different mucosal adjuvant the vaccine will stimulate the differentiation of naive $B$ and $T$ cells and induce a local mucosal immune response.

The work completed in this dissertation highlights the need to re-evaluate a system when the current strategies cannot address the problem. The development of the next generation of $B$. pertussis vaccines must improve upon waning immunity of DTaP/Tdap immunizations. It is the consensus of current literature that the most applicable way to do so is through mimicking the natural infection. To accomplish this goal researchers will need to continue to optimize systems that have reached their potential. To do so will require innovative methods, that are outside the realm of previous strategies. In this work, we demonstrate the optimization of the mouse model of pertussis to implement testing of future $B$. pertussis antigens and demonstrate the incorporation of a protective ACT toxoid into an acellular pertussis vaccine. We then altered the route of administration of the DTaP vaccine to a mucosal delivered vaccine and demonstrated the additive effects of generating a local mucosal immune response, capable of neutralizing $B$. pertussis toxins at the site of infection. This work establishes a foundation for future vaccine development, demonstrating the potential for improvements of DTaP/Tdap vaccines through the inclusion of RTX and intranasal delivery of pertussis vaccines. 


\section{References}

1 Plotkin SA, Plotkin SL. The development of vaccines: how the past led to the future. Nat Rev Microbiol 2011;9:889-93. https://doi.org/10.1038/nrmicro2668.

2 Organization WH. Pertussis Vaccines: WHO position paper-August 2015 2018:145-68.

$3 \quad$ Kendrick PL. Can Whooping Cough Be Eradicated? J Infect Dis 1975;132:70712. https://doi.org/10.1093/infdis/132.6.707.

$4 \quad$ CDC, Ncird. 2012 Final Pertussis Surveillance Report / 62(33). 2013.

$5 \quad$ Kendrick PL, Eldering G, Dixon MK, Misner J. Mouse Protection Tests in the Study of Pertussis Vaccine: A Comparative Series Using the Intracerebral Route for Challenge. Am J Public Heal Nations Heal 1947;37:803-10.

6 Guiso N, Capiau C, Carletti G, Poolman J, Hauser P. Intranasal murine model of Bordetella pertussis infection. I. Prediction of protection in human infants by acellular vaccines. Vaccine 1999;17:2366-76. https://doi.org/10.1016/S0264410X(99)00037-7.

7 De Gouw D, De Jonge MI, Hermans PWM, Wessels HJCT, Zomer A, Berends A, et al. Proteomics-identified Bvg-activated autotransporters protect against Bordetella pertussis in a mouse model. PLoS One 2014;9:e105011. https://doi.org/10.1371/journal.pone.0105011.

8 Wilk MM, Borkner L, Misiak A, Curham L, Allen AC, Mills KHG. Immunization with whole cell but not acellular pertussis vaccines primes CD4 TRM cells that sustain protective immunity against nasal colonization with Bordetella pertussis . Emerg Microbes Infect 2019;8:169-85. https://doi.org/10.1080/22221751.2018.1564630.

9 Confer DL, Eaton JW. Phagocyte impotence caused by an invasive bacterial adenylate cyclase. Science 1982;217:948-50.

10 Khelef N, Zychlinsky A, Guiso N. Bordetella pertussis induces apoptosis in macrophages: Role of adenylate cyclase-hemolysin. Infect Immun 1993;61:406471.

11 Eby JC, Gray MC, Hewlett EL. Cyclic AMP-mediated suppression of neutrophil extracellular trap formation and apoptosis by the Bordetella pertussis adenylate cyclase toxin. Infect Immun 2014;82:5256-69. https://doi.org/10.1128/IAI.0248714.

12 Mills KHG, Ross PJ, Allen AC, Wilk MM. Do we need a new vaccine to control the re-emergence of pertussis? Trends Microbiol 2014;22:49-52.

https://doi.org/10.1016/j.tim.2013.11.007.

13 Cheung GYC, Xing D, Prior S, Corbel MJ, Parton R, Coote JG. Effect of different forms of adenylate cyclase toxin of Bordetella pertussis on protection afforded by an acellular pertussis vaccine in a murine model. Infect Immun 2006;74:6797805. https://doi.org/10.1128/IAI.01104-06. 
14 Ross PJ, Sutton CE, Higgins S, Allen AC, Walsh K, Misiak A, et al. Relative contribution of Th1 and Th17 cells in adaptive immunity to Bordetella pertussis : towards the rational design of an improved acellular pertussis vaccine. PLoS Pathog 2013;9:e1003264. https://doi.org/10.1371/journal.ppat.1003264.

15 Celentano LP, Massari M, Paramatti D, Salmaso S, Tozzi AE, EUVAC-NET Group. Resurgence of pertussis in Europe. Pediatr Infect Dis J 2005;24:761-5.

16 Klein SL, Flanagan KL. Sex differences in immune responses 2016. https://doi.org/10.1038/nri.2016.90.

17 Fink AL, Klein SL. The evolution of greater humoral immunity in females than males: implications for vaccine efficacy. Curr Opin Physiol 2018;6:16-20. https://doi.org/10.1016/j.cophys.2018.03.010.

18 Hendrikx LH, Öztürk K, de Rond LGH, de Greeff SC, Sanders EAM, Berbers GAM, et al. Serum IgA Responses against Pertussis Proteins in Infected and Dutch wP or aP Vaccinated Children: An Additional Role in Pertussis Diagnostics. PLoS One 2011;6:e27681. https://doi.org/10.1371/journal.pone.0027681.

19 Wilk MM, Misiak A, McManus RM, Allen AC, Lynch MA, Mills KHG. Lung CD4 Tissue-Resident Memory T Cells Mediate Adaptive Immunity Induced by Previous Infection of Mice with Bordetella pertussis . J Immunol 2017;199:233-43. https://doi.org/10.4049/jimmunol.1602051.

20 Warfel JM, Zimmerman LI, Merkel TJ. Acellular pertussis vaccines protect against disease but fail to prevent infection and transmission in a nonhuman primate model. Proc Natl Acad Sci 2014;111:787-92. https://doi.org/10.1073/pnas.1314688110.

21 Warfel JM, Beren J, Merkel TJ. Airborne transmission of Bordetella pertussis . J Infect Dis 2012;206:902-6. https://doi.org/10.1093/infdis/jis443.

22 Warfel JM, Zimmerman LI, Merkel TJ. Acellular pertussis vaccines protect against disease but fail to prevent infection and transmission in a nonhuman primate model. Proc Natl Acad Sci 2014;111:787-92. https://doi.org/10.1073/pnas.1314688110.

23 Wendelboe AM, Van Rie A, Salmaso S, Englund JA. Duration of Immunity Against Pertussis After Natural Infection or Vaccination. Pediatr Infect Dis J 2005;24:S58-61. https://doi.org/10.1097/01.inf.0000160914.59160.41.

24 Brummelman J, Wilk MM, Han WGH, van Els CACM, Mills KHG. Roads to the development of improved pertussis vaccines paved by immunology. Pathog Dis 2015;73:ftv067. https://doi.org/10.1093/femspd/ftv067.

25 Raeven RHM, Brummelman J, van der Maas L, Tilstra W, Pennings JLA, Han WGH, et al. Immunological Signatures after Bordetella pertussis Infection Demonstrate Importance of Pulmonary Innate Immune Cells. PLoS One 2016;11:e0164027. https://doi.org/10.1371/journal.pone.0164027.

26 Skerry CM, Mahon BP. A live, attenuated Bordetella pertussis vaccine provides 
long-term protection against virulent challenge in a murine model. Clin Vaccine Immunol 2011;18:187-93. https://doi.org/10.1128/CVI.00371-10.

27 Thorstensson R, Trollfors B, Al-Tawil N, Jahnmatz M, Bergström J, Ljungman M, et al. A phase I clinical study of a live attenuated Bordetella pertussis vaccine BPZE1; a single centre, double-blind, placebo-controlled, dose-escalating study of BPZE1 given intranasally to healthy adult male volunteers. PLoS One 2014;9:e83449. https://doi.org/10.1371/journal.pone.0083449.

28 Solans L, Debrie A-S, Borkner L, Aguiló N, Thiriard A, Coutte L, et al. IL-17dependent SIgA-mediated protection against nasal Bordetella pertussis infection by live attenuated BPZE1 vaccine. Mucosal Immunol 2018;11:1753-62. https://doi.org/10.1038/s41385-018-0073-9.

29 Mielcarek N, Debrie A-S, Raze D, Bertout J, Rouanet C, Younes A Ben, et al. Live Attenuated B. pertussis as a Single-Dose Nasal Vaccine against Whooping Cough. PLoS Pathog 2006;2:e65. https://doi.org/10.1371/journal.ppat.0020065.

30 Locht C, Papin JF, Lecher S, Debrie A-S, Thalen M, Solovay K, et al. Live Attenuated Pertussis Vaccine BPZE1 Protects Baboons Against Bordetella pertussis Disease and Infection. J Infect Dis 2017;216:117-24. https://doi.org/10.1093/infdis/jix254.

31 Ross PJ, Sutton CE, Higgins S, Allen AC, Walsh K, Misiak A, et al. Relative contribution of Th1 and Th17 cells in adaptive immunity to Bordetella pertussis : towards the rational design of an improved acellular pertussis vaccine. PLoS Pathog 2013;9:e1003264. https://doi.org/10.1371/journal.ppat.1003264.

32 Kapil $\mathrm{P}$, Merkel TJ. Pertussis vaccines and protective immunity. Curr Opin Immunol 2019;59:72-8. https://doi.org/https://doi.org/10.1016/j.coi.2019.03.006.

33 Higgs R, Higgins SC, Ross PJ, Mills KHG. Immunity to the respiratory pathogen Bordetella pertussis . Mucosal Immunol 2012;5:485-500. https://doi.org/10.1038/mi.2012.54.

34 Ahmed SS, Ellis RW, Rappuoli R. Technologies for Making New Vaccines. In: Plotkin SA, OrenStein WA, Offit PA, editors. Plotkin's Vaccines. Seventh. Philadelphia, PA: Elsevier; 2018. p. 1343-53.

35 Mann JF, Acevedo R, Campo J del, Pérez O, Ferro VA. Delivery systems: a vaccine strategy for overcoming mucosal tolerance? Expert Rev Vaccines 2009;8:103-12. https://doi.org/10.1586/14760584.8.1.103.

36 Rhee JH, Lee SE, Kim SY. Mucosal vaccine adjuvants update. Clin Exp Vaccine Res 2012;1:50-63. https://doi.org/10.7774/cevr.2012.1.1.50.

37 Gringhuis SI, den Dunnen J, Litjens M, van der Vlist M, Wevers B, Bruijns SCM, et al. Dectin-1 directs $T$ helper cell differentiation by controlling noncanonical NF[kappa]B activation through Raf-1 and Syk. Nat Immunol 2009;10:203-13. https://doi.org/10.1038/ni.1692.

38 Agrawal S, Gupta S, Agrawal A. Human dendritic cells activated via dectin-1 are 
efficient at priming th17, cytotoxic CD8 T and B cell responses. PLoS One 2010;5:e13418. https://doi.org/10.1371/journal.pone.0013418.

39 Higashi T, Hashimoto K, Takagi R, Mizuno Y, Okazaki Y, Tanaka Y, et al. Curdlan Induces DC-Mediated Th17 Polarization via Jagged1 Activation in Human Dendritic Cells. Allergol Int 2010;59:161-6. https://doi.org/10.2332/allergolint.09OA-0103.

40 Wu W, Huang J, Duan B, Traficante DC, Hong H, Risech M, et al. Th17stimulating protein vaccines confer protection against Pseudomonas aeruginosa pneumonia. Am J Respir Crit Care Med 2012;186:420-7. https://doi.org/10.1164/rccm.201202-01820C.

41 Neutra MR, Kozlowski PA. Mucosal vaccines: the promise and the challenge. Nat Rev Immunol 2006;6:148-58.

42 Yusuf $\mathrm{H}$, Kett $\mathrm{V}$. Current prospects and future challenges for nasal vaccine delivery. Hum Vaccin Immunother 2017;13:. https://doi.org/10.1080/21645515.2016.1239668.

43 Illum L. Nanoparticulate Systems for Nasal Delivery of Drugs: A Real Improvement over Simple Systems? J Pharm Sci 2007;96:473-83. https://doi.org/10.1002/jps.20718.

44 Southam DS, Dolovich M, O'Byrne PM, Inman MD. Distribution of intranasal instillations in mice: effects of volume, time, body position, and anesthesia. Am J Physiol Cell Mol Physiol 2002;282:L833-9. https://doi.org/10.1152/ajplung.00173.2001.

45 Miller MA, Stabenow JM, Parvathareddy J, Wodowski AJ, Fabrizio TP, Bina XR, et al. Visualization of murine intranasal dosing efficiency using luminescent Francisella tularensis: effect of instillation volume and form of anesthesia. PLOS One 2012;7:e31359. https://doi.org/10.1371/journal.pone.0031359.

46 Raeven RHM, Brummelman J, Pennings JLA, van der Maas L, Tilstra W, Helm K, et al. Bordetella pertussis outer membrane vesicle vaccine confers equal efficacy in mice with milder inflammatory responses compared to a whole-cell vaccine. Sci Rep 2016;6:38240. https://doi.org/10.1038/srep38240.

47 Warfel JM, Merkel TJ. Bordetella pertussis infection induces a mucosal IL-17 response and long-lived Th17 and Th1 immune memory cells in nonhuman primates. Mucosal Immunol 2013;6:787-96. https://doi.org/10.1038/mi.2012.117.

48 Belshe RB, Gruber WC, Mendelman PM, Mehta HB, Mahmood K, Reisinger K, et al. Correlates of Immune Protection Induced by Live, Attenuated, Cold-Adapted, Trivalent, Intranasal Influenza Virus Vaccine. J Infect Dis 2000;181:1133-7. https://doi.org/10.1086/315323.

49 Low N, Kraemer S, Schneider M, Restrepo AMH. Immunogenicity and safety of aerosolized measles vaccine: Systematic review and meta-analysis. Vaccine 2008;26:383-98. https://doi.org/10.1016/j.vaccine.2007.11.010. 
50 Rohani P, Scarpino S V. Pertussis : epidemiology, immunology, and evolution. 1st ed. New York: Oxford University Press; 2019.

51 Solans L, Locht C. The Role of Mucosal Immunity in Pertussis. Front Immunol 2018;9:. https://doi.org/10.3389/FIMMU.2018.03068.

52 Allen AC, Wilk MM, Misiak A, Borkner L, Murphy D, Mills KHG. Sustained protective immunity against Bordetella pertussis nasal colonization by intranasal immunization with a vaccine-adjuvant combination that induces IL-17-secreting TRM cells. Mucosal Immunol 2018. https://doi.org/10.1038/s41385-018-0080-x.

53 Mattoo S, Cherry JD. Molecular pathogenesis, epidemiology, and clinical manifestations of respiratory infections due to Bordetella pertussis and other Bordetella subspecies. Clin Microbiol Rev 2005;18:326-82. https://doi.org/10.1128/CMR.18.2.326-382.2005.

54 Zurita ME, Wilk MM, Carriquiriborde F, Bartel E, Moreno G, Misiak A, et al. A Pertussis Outer Membrane Vesicle-Based Vaccine Induces Lung-Resident Memory CD4 T Cells and Protection Against Bordetella pertussis , Including Pertactin Deficient Strains. Front Cell Infect Microbiol 2019;9:125. https://doi.org/10.3389/fcimb.2019.00125.

55 Hava DL, LeMieux J, Camilli A. From nose to lung: the regulation behind Streptococcus pneumoniae virulence factors. Mol Microbiol 2003;50:1103-10.

56 Conover MS, Sloan GP, Love CF, Sukumar N, Deora R. The Bps polysaccharide of Bordetella pertussis promotes colonization and biofilm formation in the nose by functioning as an adhesin. Mol Microbiol 2010;77:1439-55. https://doi.org/10.1111/j.1365-2958.2010.07297.x.

57 Serra DO, Conover MS, Arnal L, Sloan GP, Rodriguez ME, Yantorno OM, et al. FHA-mediated cell-substrate and cell-cell adhesions are critical for Bordetella pertussis biofilm formation on abiotic surfaces and in the mouse nose and the trachea. PLoS One 2011;6:e28811. https://doi.org/10.1371/journal.pone.0028811.

58 Mc Guirk P, Mahon BP, Griffin F, Mills KHG. Compartmentalization of T cell responses following respiratory infection with Bordetella pertussis :

hyporesponsiveness of lung $T$ cells is associated with modulated expression of the co-stimulatory molecule CD28. Eur J Immunol 1998;28:153-63. https://doi.org/10.1002/(sici)1521-4141(199801)28:01<153::Aidimmu153>3.0.Co;2-\#.

59 Kirimanjeswara GS, Agosto LM, Kennett MJ, Bjornstad ON, Harvill ET. Pertussis toxin inhibits neutrophil recruitment to delay antibody-mediated clearance of Bordetella pertussis . J Clin Invest 2005;115:3594-601. https://doi.org/10.1172/JCl24609.

60 Ong SE, Mann M. Mass Spectrometry-Based Proteomics Turns Quantitative. Nat Chem Biol 2005;1:252-62. https://doi.org/10.1038/nchembio736.

61 Eby JC, Hoffman CL, Gonyar LA, Hewlett EL. Review of the neutrophil response 
to Bordetella pertussis infection. Pathog Dis 2015;73:ftv081. https://doi.org/10.1093/femspd/ftv081.

62 Kapil P, Papin JF, Wolf RF, Zimmerman LI, Wagner LD, Merkel TJ. Maternal Pertussis Vaccination • JID 2018:217 (15 April) • 1231 The Journal of Infectious Diseases n.d. https://doi.org/10.1093/infdis/jiy022.

63 Thierry-Carstensen B, Dalby T, Stevner MA, Robbins JB, Schneerson R, Trollfors B. Experience with monocomponent acellular pertussis combination vaccines for infants, children, adolescents and adults--a review of safety, immunogenicity, efficacy and effectiveness studies and 15 years of field experience. Vaccine 2013;31:5178-91. https://doi.org/10.1016/j.vaccine.2013.08.034.

64 Carbonetti NH, Artamonova G V., Andreasen C, Bushar N. Pertussis toxin and adenylate cyclase toxin provide a one-two punch for establishment of Bordetella pertussis infection of the respiratory tract. Infect Immun 2005;73:2698-703. https://doi.org/10.1128//AI.73.5.2698-2703.2005.

65 Carbonetti NH, Artamonova G V, Mays RM, Worthington ZE V. Pertussis toxin plays an early role in respiratory tract colonization by Bordetella pertussis . Infect Immun 2003;71:6358-66. https://doi.org/10.1128/iai.71.11.6358-6366.2003.

66 Conover MS, Redfern CJ, Ganguly T, Sukumar N, Sloan G, Mishra M, et al. BpsR modulates Bordetella biofilm formation by negatively regulating the expression of the Bps polysaccharide. J Bacteriol 2012;194:233-42. https://doi.org/10.1128/JB.06020-11.

67 Thanabalasuriar A, Scott BNV, Peiseler M, Willson ME, Zeng Z, Warrener P, et al. Neutrophil Extracellular Traps Confine Pseudomonas aeruginosa Ocular Biofilms and Restrict Brain Invasion. Cell Host Microbe 2019;25:526-536.e4. https://doi.org/10.1016/j.chom.2019.02.007.

68 Zhang X, Goel T, Goodfield LL, Muse SJ, Harvill ET. Decreased Leukocyte Accumulation and Delayed Bordetella pertussis Clearance in IL-6-/- Mice. J Immunol 2011;186:4895-904. https://doi.org/10.4049/jimmunol.1000594.

69 Dunne A, Mielke LA, Allen AC, Sutton CE, Higgs R, Cunningham CC, et al. A novel TLR2 agonist from Bordetella pertussis is a potent adjuvant that promotes protective immunity with an acellular pertussis vaccine. Mucosal Immunol 2015;8:607-17. https://doi.org/10.1038/mi.2014.93.

70 Bottero D, Gaillard ME, Zurita E, Moreno G, Martinez DS, Bartel E, et al. Characterization of the immune response induced by pertussis OMVs-based vaccine. Vaccine 2016;34:3303-9. https://doi.org/10.1016/j.vaccine.2016.04.079.

71 Raeven RHM, Van Der Maas L, Tilstra W, Uittenbogaard JP, Bindels THE, Kuipers B, et al. Immunoproteomic Profiling of Bordetella pertussis Outer Membrane Vesicle Vaccine Reveals Broad and Balanced Humoral Immunogenicity. J Proteome Res 2015;14:2929-42. https://doi.org/10.1021/acs.jproteome.5b00258. 
72 Garlapati S, Eng NF, Kiros TG, Kindrachuk J, Mutwiri GK, Hancock REW, et al. Immunization with PCEP microparticles containing pertussis toxoid, CpG ODN and a synthetic innate defense regulator peptide induces protective immunity against pertussis. Vaccine 2011;29:6540-8. https://doi.org/10.1016/j.vaccine.2011.07.009.

73 Klein NP, Bartlett J, Fireman B, Baxter R. Waning Tdap Effectiveness in Adolescents. Pediatrics 2016;137:e20153326-e20153326. https://doi.org/10.1542/peds.2015-3326.

74 da Silva Antunes R, Babor M, Carpenter C, Khalil N, Cortese M, Mentzer AJ, et al. Th1/Th17 polarization persists following whole-cell pertussis vaccination despite repeated acellular boosters. J Clin Invest 2018;128:3853-65. https://doi.org/10.1172/JCl121309. 